\title{
Landlord Program Multi-Year Program Plan Fiscal Year 1995 \\ WBS 7.5
}
Prepared for the U.S. Department of Energy Office of Environmental Restoration and Waste Management

September 1994

\section{$\hat{\Rightarrow}$ ICF Kaiser Hanford Company \\ P.O Box 888 \\ Richland, Washington}

Hanford Architect and Engineering Sub-contrector for the 
LEGAL DISCLAIMER

This report was prepared as an account of work sponsored by an agency of the United States Government. Neither the

United States Government nor any agency thereof, nor any of their employees, nor any of their contractors, subcontractors or their employees, makes any warranty, express or implied, or assumes any legal liability or responsibility for the accuracy, completeness, or any third party's use or the results of such use of any information, apparatus, product, or process disclosed, or represents that its use would not infringe privately owned rights. Reference herein to any specific commercial product, process, or service by trade name, trademark, manufacturer, or otherwise, does not necessarily constitute or imply its endorsement, recommendation, or favoring by the United States Government or any agency thereof or its contractors or subcontractors. The views and opinions of authors expressed herein do not necessarily state or reflect those of the United States Government or any agency thereof.

This report has been reproduced from the best available copy.

Available to the U.S. Department of Energy and its contractors from

Office of Scientific and Technical Information P.O. Box 62

Oak Ridge, TN 37831

(615) $576-8401$

Prinied in the United States of America 


\section{DISCLAIMER}

Portions of this document may be illegible in electronic image products. Images are produced from the best available original document. 


\section{Landlord Program Multi-Year Program Plan Fiscal Year 1995 WBS 7.5}

\section{MYPP Approval Sheet}

Assistant Manager-Contracting Officer's Representative

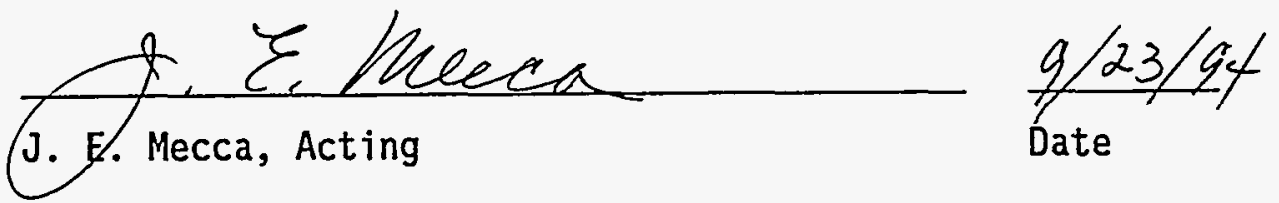

Director, Waste, Analytical, and Environmental Services

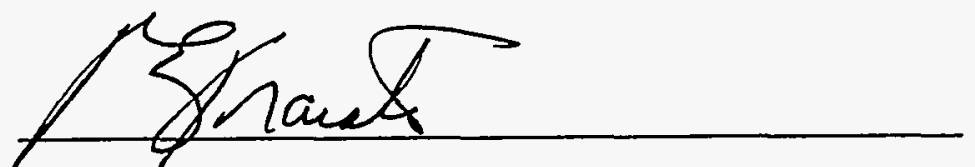

R. E. Traister, Acting

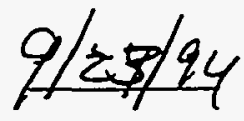

Date

\section{ICF-KH Director}

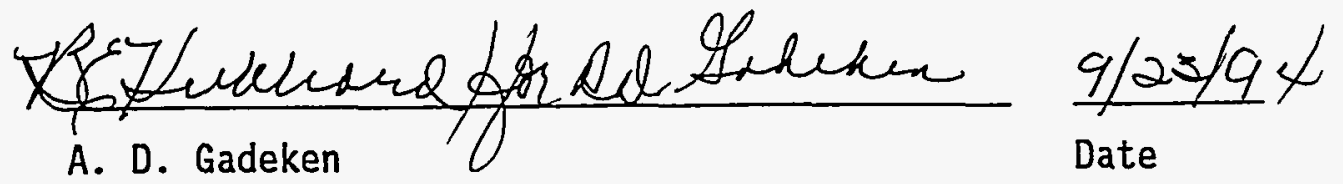


. 


\section{RELEASE AUTHORIZATION}

\section{Document Number: $\quad$ ICF-0001}

Document Title: Landlord Program Multi-Year Program Plan Fiscal Year 1995 WBS 7.5 .

Release Date: $\quad 9 / 23 / 94$.

***************

This document was reviewed following the procedures described in WHC-CM-3-4 and is:

APPROVED FOR PUBLIC RELEASE

***************

WHC Information Release Administration Specialist:

Ghriateris Btellereftad

C. Willingham

$9 / 23 / 94$

(Signature)

(Date) 
ICF-0001

\section{LANDLORD PROGRAM MULTI-YEAR PROGRAM PLAN FISCAL YEAR 1995 \\ WBS 7.5}

Table of Contents

1.0 Program Overview

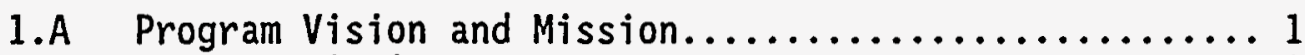

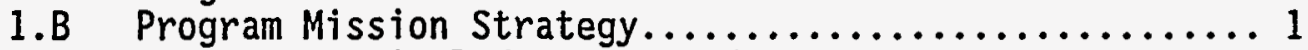
1.B.1 Technical Function and Requirements......... 2 1.B.2 Program 0bjectives.................... 3

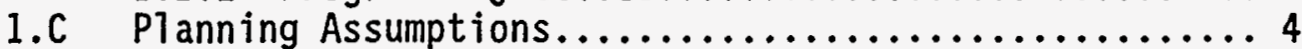

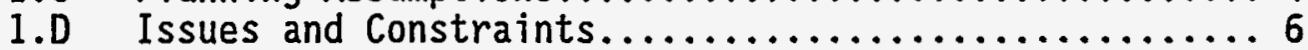

2.0 Program Baselines

2.A Work Breakdown Structure (WBS) Information

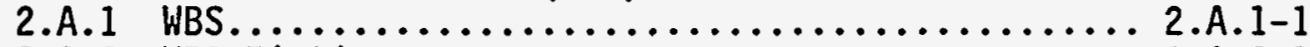

2.A.2 WBS Dictionary.......................... 2.

2.B Responsibility Assignment Matrix.................... 2.1

2.C Program Logic Diagrams............................. 2.

2.D Program Master Baseline Schedule (PMBS)............ 2.D-1

2.E Program Performance Baseline Schedule (PPBS) ......... 2.E-1

2.F Milestone List...............................

2.G Milestone Description Sheet........................ 2.1

2.H Program Cost Basel ine Summary by Year.............. 2.H-1

2.I Basis of Estimate............................

2.J Waste Type Projections.......................... 2.

2.K Planned Staffing (Ful1-Time Equivalent)...............

3.0 Fiscal Year Work Plan (FYWP)

3.A Cost Basel ine Summary...........................

3.B Program Funding by Month........................

3.C Program Funding by ADS/Fund-Type...................... 
ICF-0001

FY 1995 MYPP

\section{LANDLORD PROGRAM}

7.5

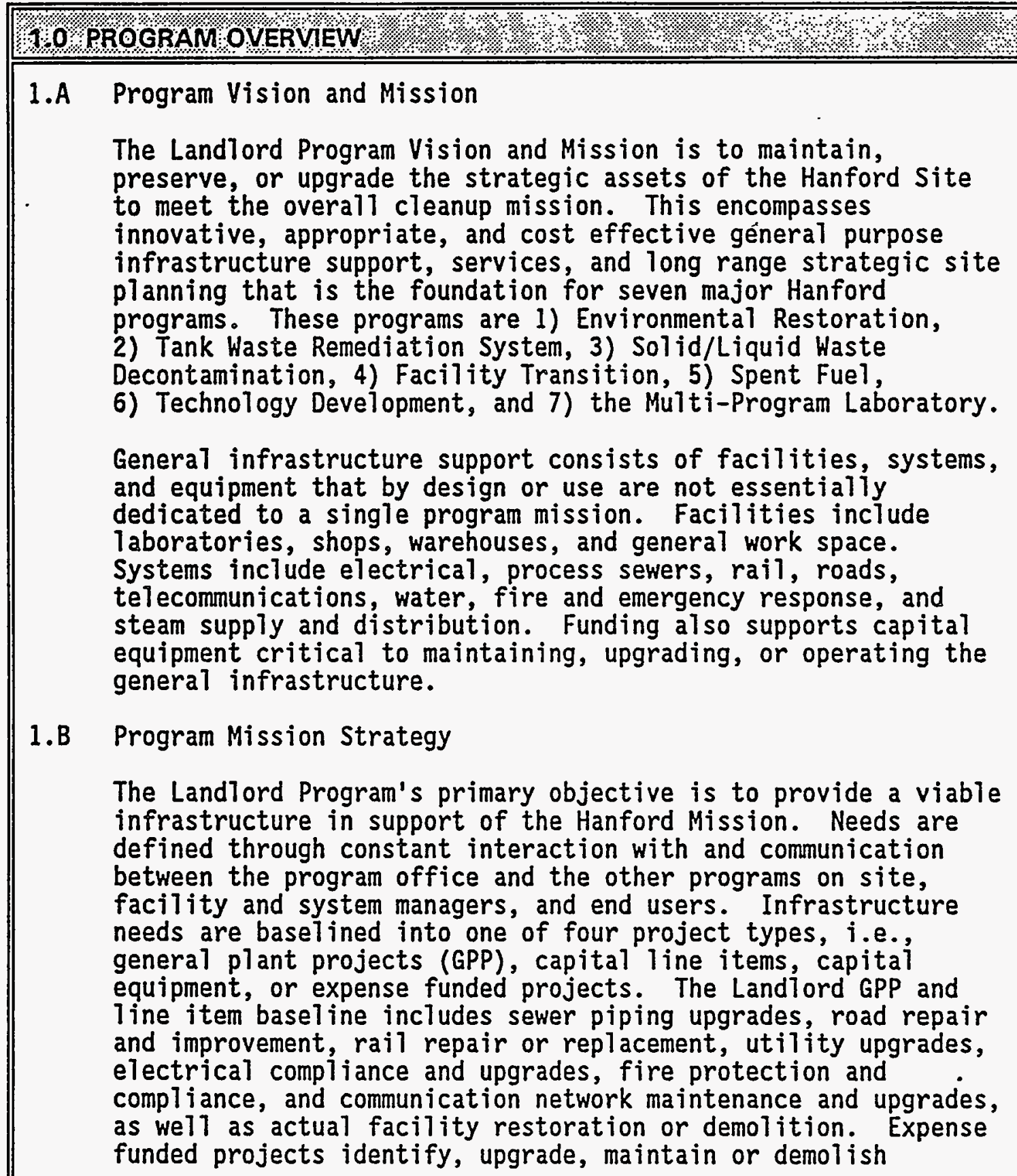


ICF-0001

LANDLORD PROGRAM

FY 1995 MYPP

7.5

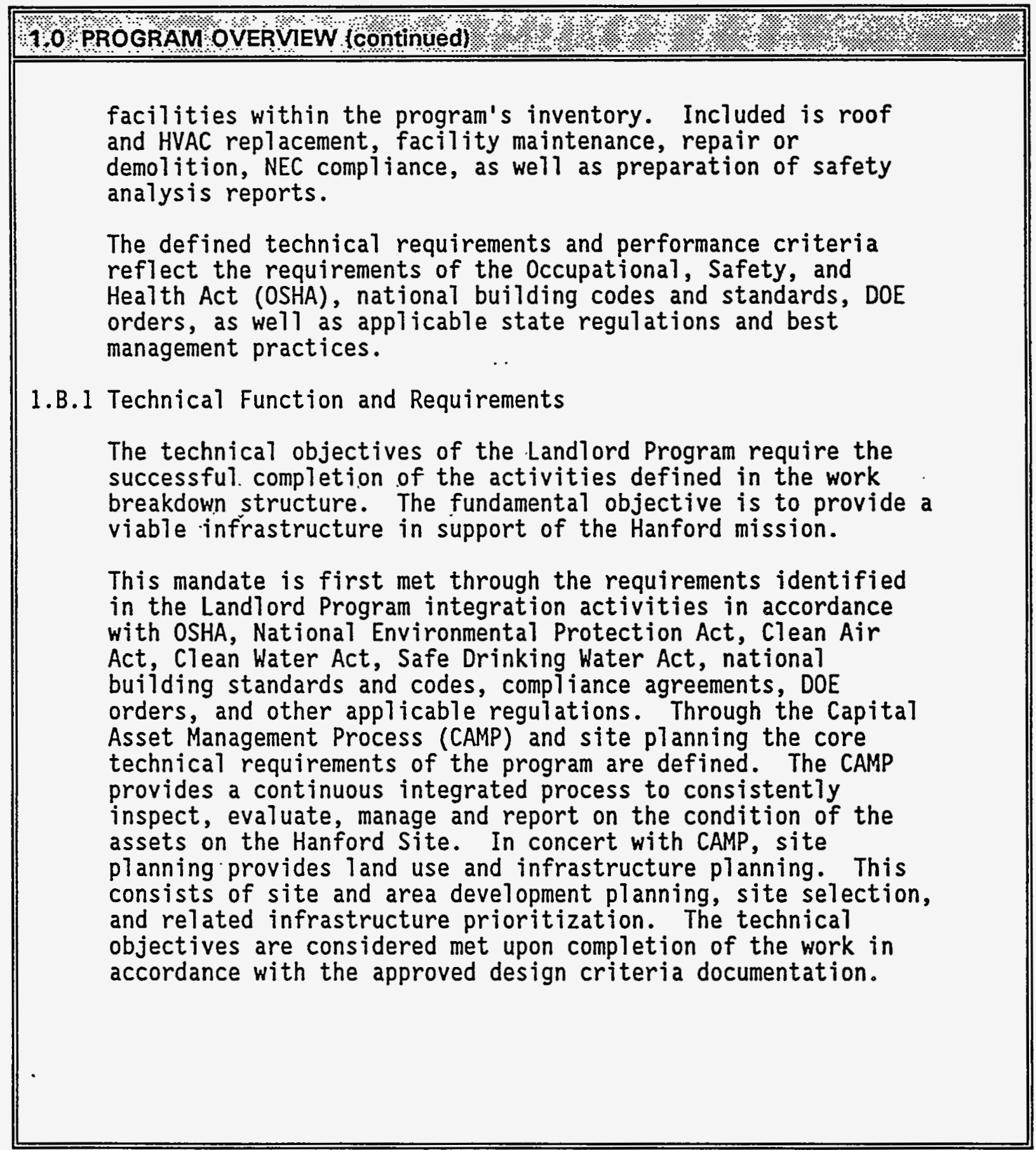


ICF -0001

\section{LANDLORD PROGRAM}

FY 1995 MYPP.

7.5

10. PROGRAM OVERVEW (continued)

1.B.2 Program Objectives

The overall Landlord Program objective is to maintain a standard of excellence and safety while providing efficient, affordable, and effective infrastructure and support services.

The Program will also strive to maintain, preserve, or upgrade strategic assets while consolidating facilities and functions to lower operating and maintenance costs. Realistic goals and strong baseline control are critical to meeting the Program objectives. Paramount to these objectives is compliance with all applicable laws, orders, agreements, codes, standards, best management and safety practices.

The objectives for general infrastructure support are reflected in five programmatic functions, 1) Program Integration,

2) Capita! Equipment, 3) Expen'se Funded Projects, 4) General. Plant Projects, and 5) Line Itéms.

The following objectives are applicable to the Landlord Program:

Provide an infrastructure that serves the Hanford mission, leave diversification value and a viable asset, concentrate service locations, upgrade or demolish inadequate and under utilized facilities, promote energy efficiency, and maintain facilities and equipment as economically and as safely as possible.

Integrate Occupational Safety and Health Act (OSHA) requirements into the continuous facility inspection program.

Ensure compliance with applicable federal and state regulations, and the Tri-Party Agreement.

Identify future infrastructure needs, integrate Landlord Program planning with other program plans, effectively use 1 imited resources, improve project definition and cost estimating, consolidate similar projects into Line Items, and direct fund all appropriate costs. 
ICF-0001

\section{LANDLORD PROGRAM}

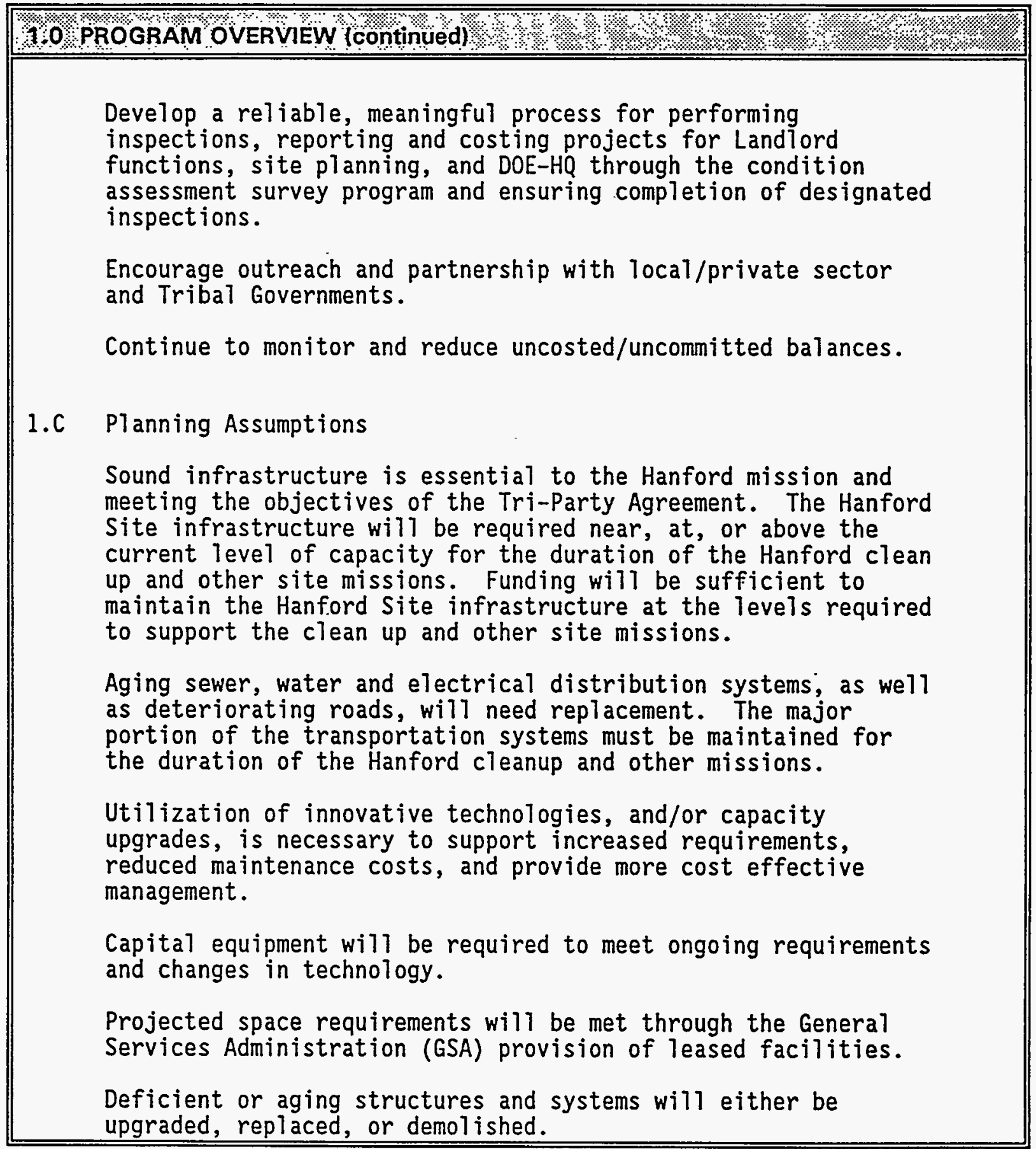


ICF-000I

\section{LANDLORD PROGRAM}

FY 1995 MYPP

$$
7.5
$$

10. RROGRAM OVERVIEw (continued)
Extensions and upgrades to infrastructure systems will be
required as the configuration of work centers across the site
adjusts with the needs of the Hanford cleanup.
An Environmental Impact Statement (EIS) is not currently
anticipated for activities or projects, i.e., all activities
will be included in either categorical exclusions or
environmental assessments.
To ensure safe operations, as well as worker safety and health,
deficient or aging structures requiring upgrade will be brought
into compliance with applicable building codes and regulations.
The Capital Asset Management Process (CAMP), in conjunction
with Site Development Planning, will serve as the basis for
overall Landlord Program priorities. Findings are integrated
with other Hanford Site programs through the development of the
Hanford Site Specific Plan.
Baseline control will be utilized to maintain financial and
programmatic accountability.
Landlord associated work scope currently funded through
indirect funding will become direct funded through the Landlord
Program beginning in FY 1997.
Potential service provision by local jurisdictions in the
Southern portion of the Hanford Site is influencing capital
expenditure decisions for utilities and other general purpose
facilities. It is assumed that commercial private enterprises
will provide needed infrastructure components at a lesser cost
to the Hanford Site. Note: No plan currently exists to
transfer land or facilities where infrastructure is an issue.
Carryover funding will be available to fund carryover work
scope as required.
The Landlord Program cost basel ine summary for Fy 2001 was
derived by escalating FY 2000 by 3 percent per verbal direction
from Westinghouse Hanfod Company Planning Integration.


- ICF-0001

\section{LANDLORD PROGRAM}

\begin{tabular}{|c|c|}
\hline 1.D & 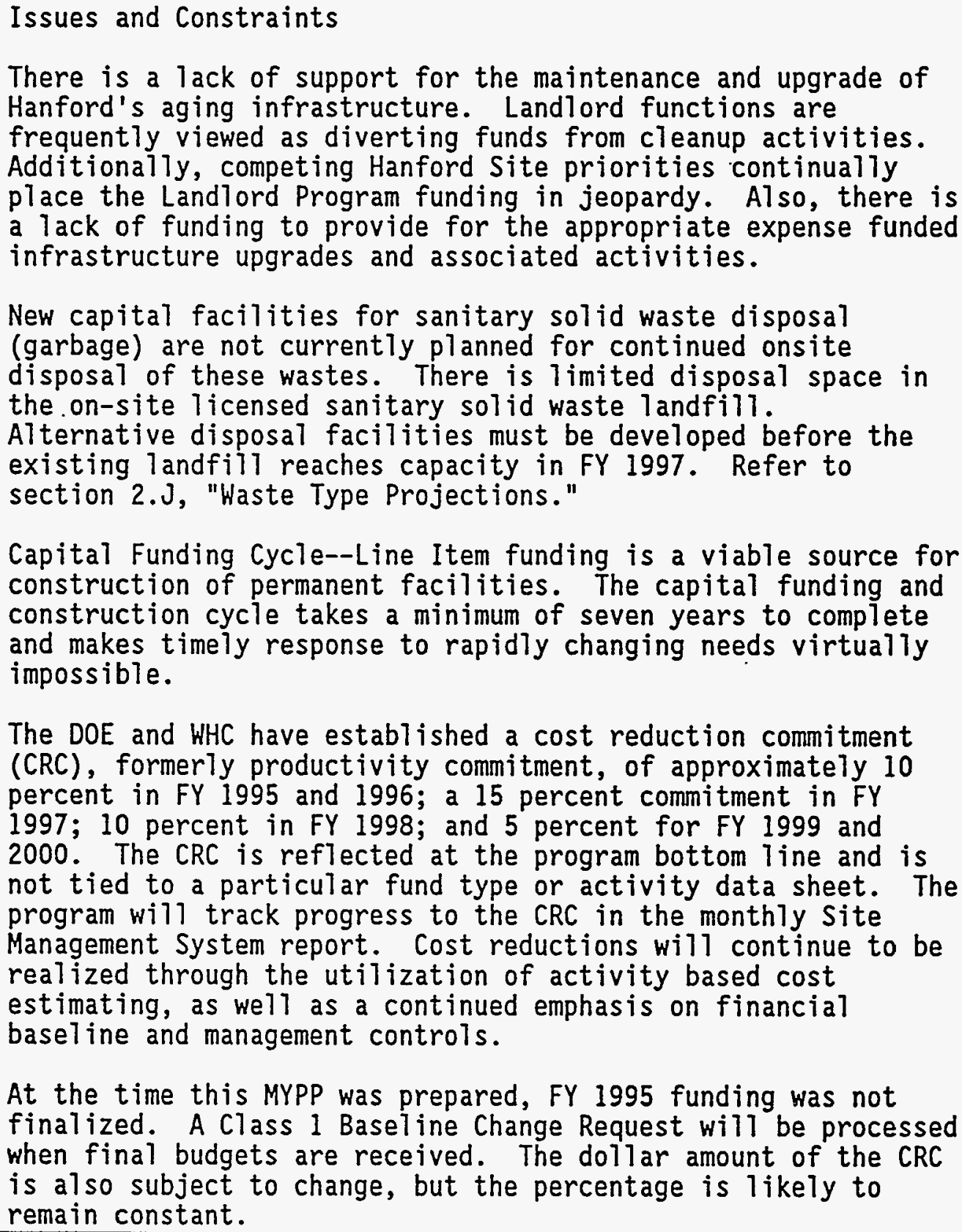 \\
\hline
\end{tabular}




\section{LANDLORD PROGRAM}

\section{FY 1995 MYPP}

\section{5}

\section{A.1 WORK BREAKBOWN STRGUTURE}

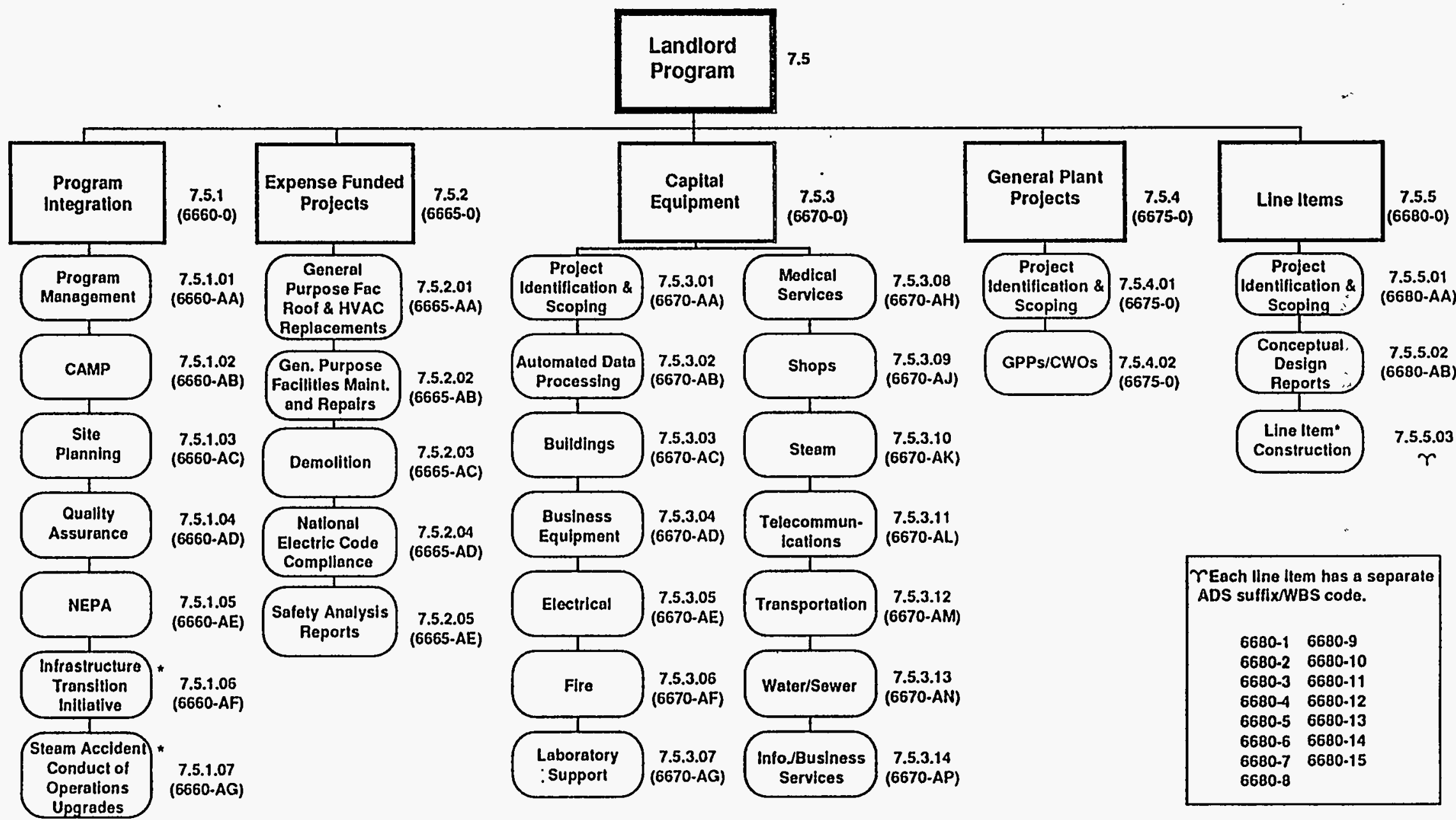

* FY 1994 funding only 


\section{LANDLORD PROGRAM}

\section{5}

2.A.1 Work Breakdown Structure Index and Programmatic Responsibi] ity Ass ignment latrix Responsible organization legend:

$\begin{array}{ll}\text { BM } & \text { Business Management } \\ \text { CAMP } & \text { Capital Asset Management Process } \\ \text { CS } & \text { Calibration Services } \\ \text { CHO } & \text { Capital Work Order } \\ \text { EP } & \text { Emergency Preparedness } \\ \text { ENG } & \text { Engineering } \\ \text { FM } & \text { Facilities Management } \\ \text { GPP } & \text { General Plant Projects } \\ \text { HEHF } & \text { Hanford Environmental Health Foundation } \\ \text { HFD } & \text { Hanford Fire Department } \\ \text { HVAC } & \text { Heating Ventilation and Air Conditioning } \\ \text { LPM } & \text { Landlord Program Management } \\ \text { LP } & \text { Landlord Projects } \\ \text { LSP } & \text { Leasing and Special Projects } \\ \text { MPI } & \text { Maintenance Programs Integration } \\ \text { NEPA } & \text { National Environmental Protection Act (NEPA) Services } \\ \text { PNL } & \text { Pacific Northwest Laboratories (Battelle) } \\ \text { QA } & \text { Quality Assurance } \\ \text { SP } & \text { Site Planning } \\ \text { SARP } & \text { Steam Accident Recovery Program } \\ \text { TEL } & \text { Telecommunications } \\ \text { TFS } & \text { Transportation and Fabrication Services } \\ \text { UTL } & \text { Utilities } \\ & \end{array}$




\section{LANDLORD PROGRAM}

FY 1995 MYPP

$$
7.5
$$

\begin{tabular}{|c|c|c|c|}
\hline \multicolumn{3}{|l|}{2 A.2 WBS Dictionary } & \\
\hline \multicolumn{4}{|c|}{ WORK BREAKDOWN STRUCTURE DICTIONARY } \\
\hline \multicolumn{2}{|c|}{$\begin{array}{l}\text { I PROJECT TITLE/PARTICIPANT } \\
\text { Landlord Program / ICF Kaiser Hanford }\end{array}$} & $\begin{array}{l}2 \text { DATE } \\
\text { August } 31,1994\end{array}$ & $\begin{array}{l}3 \text { IDENTIFICATION NO. } \\
\text { ADS } 6660-0\end{array}$ \\
\hline \multicolumn{2}{|l|}{$\begin{array}{l}4 \text { UBS ELEMENT COOE } \\
7.5 .1\end{array}$} & \multicolumn{2}{|l|}{$\begin{array}{l}5 \text { WBS ELEMENT TITLE } \\
\text { Program integration }\end{array}$} \\
\hline $\begin{array}{l}6 \text { INDEX LINE NO. } \\
1.6 .8 .2 .5 .1\end{array}$ & 7 REVISIO & AND AUTHORIZATION & 8 DATE \\
\hline
\end{tabular}

9 APPROVED CHANGES

\begin{tabular}{|l|l}
\hline 10 SYSTEM DESIGN DESCRIPTION & 11 BUDGET AND REPORTING NUMBER \\
Program Integration & EH70300000
\end{tabular}

12 ELEMENT TASK DESCRIPTION

A. COST CONTENT

This WBS is labor intensive. Also included are office supplies and other materials, mandatory computer upgrades and software, essential travel and training, Information Resources Management systems support.

\section{B. TECHHICAL CONTENT/WORK STATEMENT}

This HBS of the Hanford Landlord Program provides program integration activities for the Landlord Program and in support of the Hanford Site. Included are Program Management, Capital Asset Management Process (CAMP), site Planning, and National Environmental Policy Act (NEPA) Documentation. Funding is required for these Program Integration activities to ensure the site wide infrastructure is adequately managed. To safely fulfill the present and future mission requirements at the Hanford site, a sound basic infrastructure to maintain essential services is necessary. Infrastructure is defined as "the basic facilities, equipment, and installations needed in order for the systems/operations of the Hanford site to properly function." 
ICF-0001

\section{LANDLORD PROGRAM}

FY 1995 MYPP

7.5

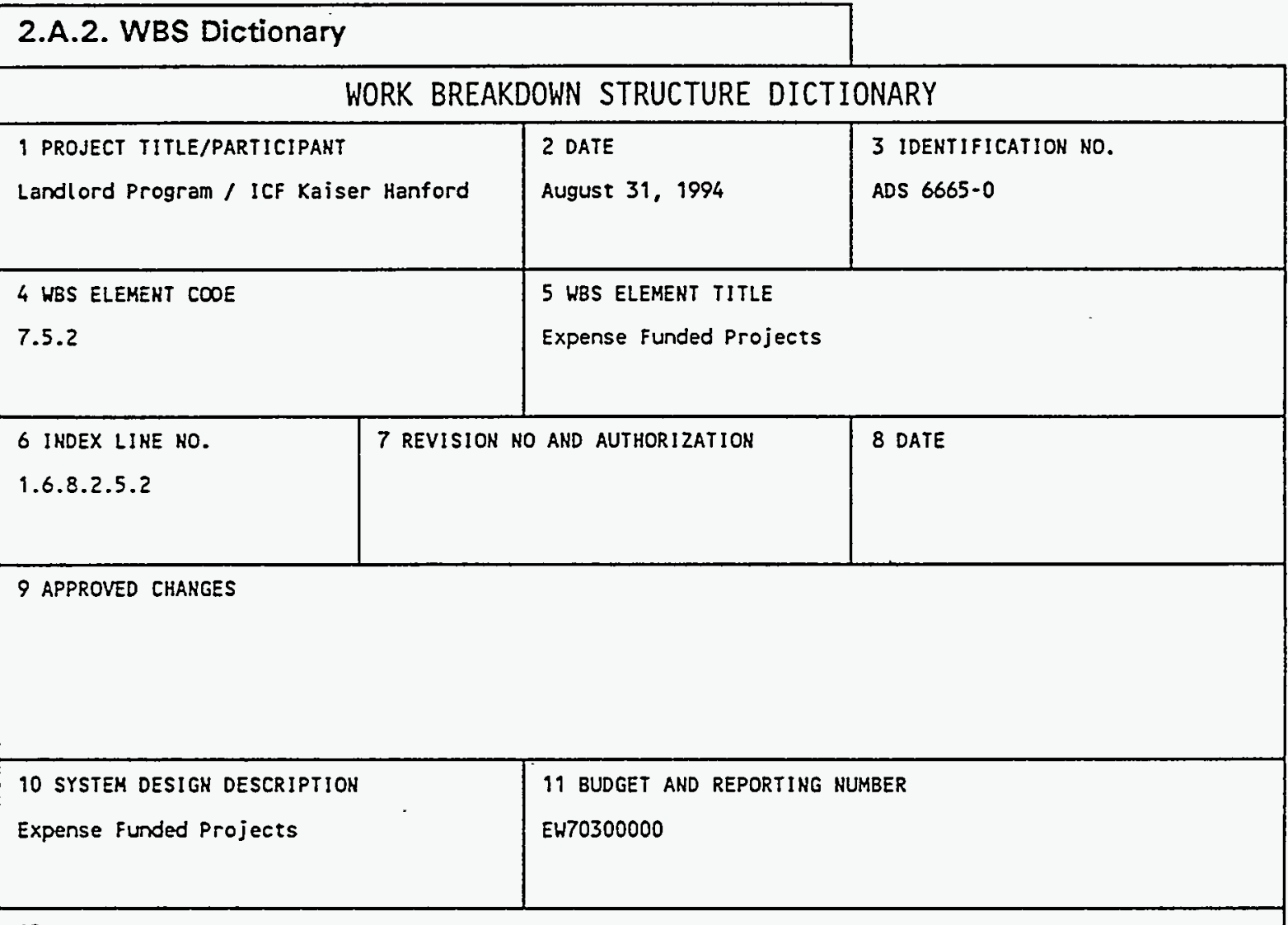

12 ELEMENT TASK DESCRIPTION

A. COST CONTENT

This WBS element contains construction, engineering, project management and inspection. Most construction is offsite/fixed-priced contract labor, equipment and materials.

\section{B. TECHNICAL CONTENT/WORK STATEMENT}

This WBS of the Hanford Landlord Program provides for expense related construction, demolition and maintenance to assure a viable infrastructure for the overall site mission of Waste Management and Envirormental Restoration. Included in Expense Funded Projects is 1) General Purpose Facility (GPF) roof replacement and heating, ventilating and air conditioning (HVAC) replacement; 2) GPF maintenance and repair; 3) GPF demolition; 4) GPF National Electric Code (NEC) compliance upgrades and; 5) Safety Analysis Report preparation for Pacific Northwest Laboratory operated GPFs. Support of the Hanford

site infrastructure is the primary mission of the Hanford Landlord Program. 


\section{LANDLORD PROGRAM}

FY 1995 MYPP

7.5

\begin{tabular}{|c|c|c|c|}
\hline \multicolumn{3}{|l|}{ 2.A.2. WBS Dictionary } & \\
\hline \multicolumn{4}{|c|}{ WORK BREAKDOWW STRUCTURE DICTIONARY } \\
\hline \multicolumn{2}{|c|}{$\begin{array}{l}1 \text { PROJECT TITLE/PARTICIPANT } \\
\text { Landlord Program / ICF Kaiser Hanford }\end{array}$} & $\begin{array}{l}2 \text { DATE } \\
\text { August 31, } 1994\end{array}$ & $\begin{array}{l}3 \text { IDENTIFICATION NO. } \\
\text { ADS } 6670-0\end{array}$ \\
\hline \multicolumn{2}{|l|}{$\begin{array}{l}4 \text { WBS ELEMENT COOE } \\
7.5 .3\end{array}$} & \multicolumn{2}{|c|}{$\begin{array}{l}5 \text { HBS ELEMENT TITLE } \\
\text { Capital Equipment Not Related to Construction (CENRTC) }\end{array}$} \\
\hline $\begin{array}{l}6 \text { IHDEX LINE NO. } \\
1.6 .8 .2 .5 .3\end{array}$ & 7 REVISI & AND AUTHORIZATION & 8 DATE \\
\hline \multicolumn{4}{|l|}{9 APPROVED CHANGES } \\
\hline \multicolumn{2}{|l|}{$\begin{array}{l}10 \text { SYSTEM OESIGH DESCRIPTION } \\
\text { Capital Equipment }\end{array}$} & \multicolumn{2}{|c|}{$\begin{array}{l}11 \text { BUDGET AND REPORTING NUMBER } \\
35 \text { E } 703000\end{array}$} \\
\hline \multicolumn{4}{|c|}{$\begin{array}{l}12 \text { ELEMENT TASK DESCRIPTION } \\
\text { A. COST CONTENT } \\
\text { This WBS element contains materials and the applicable material procurement rate, and beginning with } \\
\text { FY } 1995 \text { general and administrative (G\&A) rate. In some CENRTC activities labor for design and } \\
\text { installation is also included, i.e., steam, telecommunication, and water/sewer. }\end{array}$} \\
\hline \multicolumn{4}{|c|}{$\begin{array}{l}\text { B. TECHNICAL CONTENT/HORK STATEMENT } \\
\text { This WBS of the Hanford Landlord Program provides for general plant equipment replacements of existing } \\
\text { equipment which support the infrastructure of the Hanford Site. Equipment replacements are required } \\
\text { to maintain safe facility operations and support ongoing activities at the Hanford site. } \\
\text { As the site increases in age, we are finding that the infrastructure is decaying at a faster rate than } \\
\text { available funding Hill support. Areas that are in greatest need of replacements include water/sewer, } \\
\text { electrical, } 300 \text { Area steam, fabrication shops, transportation and business services. Also, with the } \\
\text { increased growth in the site mission at Hanford, the infrastructure must expand to support this growth } \\
\text { especially in the telecommications, ADP and support services area. }\end{array}$} \\
\hline
\end{tabular}


ICF-0001

\section{LANDLORD PROGRAM}

FY 1995 MYPP

7.5

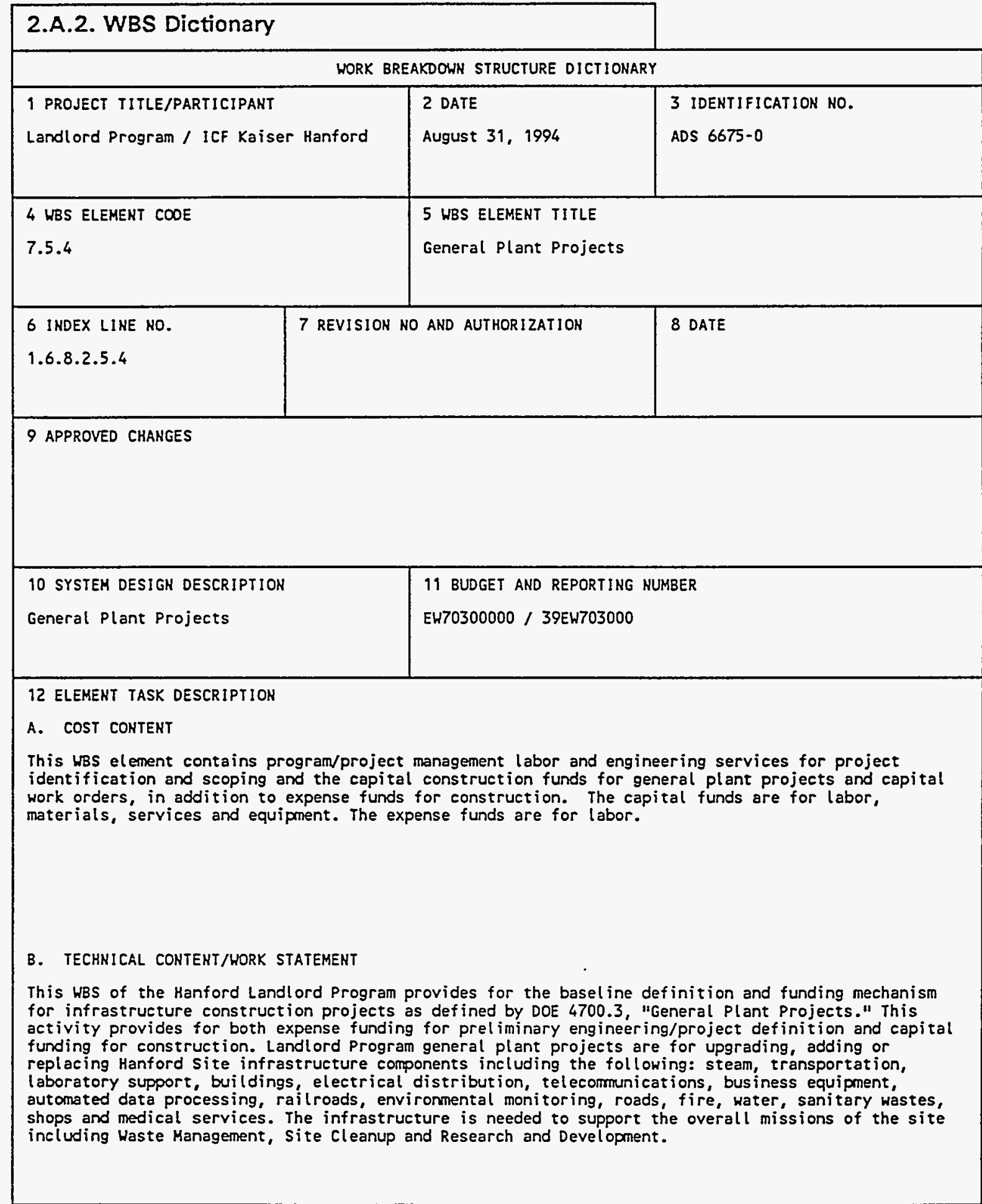


ICF-0001

\section{LANDLORD PROGRAM}

FY 1995 MYPP

7.5

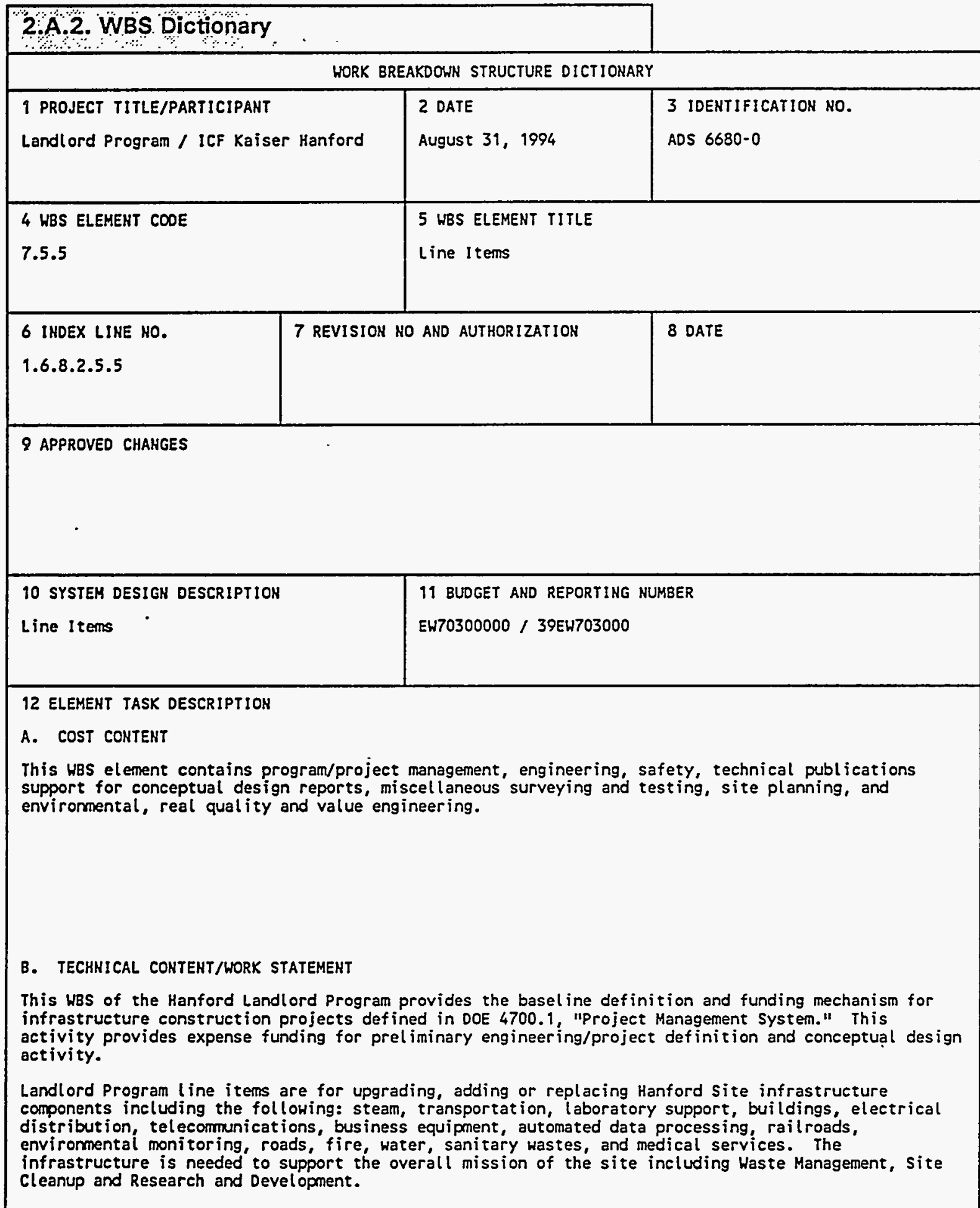


ICF-0009

\section{LANDLORD PROGRAM}

FY 1995 MYPP

7.5

\section{A.2. WBS Dictionary}

WORK BREAKDOWN STRUCTURE DICTIONARY

\begin{tabular}{|c|c|c|c|}
\hline $\begin{array}{l}1 \text { PROJECT TITLE/PARTICIPANT } \\
\text { Landlord Program / ICF Kaiser }\end{array}$ & Hanford & $\begin{array}{l}2 \text { DATE } \\
\text { August } 31,1994\end{array}$ & $\begin{array}{l}3 \text { IDENTIFICATION NO. } \\
\text { ADS } 6680-01\end{array}$ \\
\hline $\begin{array}{l}4 \text { WBS ELEMENT CODE } \\
7.5 .5 .03\end{array}$ & & $\begin{array}{l}5 \text { WBS ELEMENT TITLE } \\
300 \text { Area Multi-Purpose }\end{array}$ & ility \\
\hline $\begin{array}{l}6 \text { INDEX LINE NO. } \\
1.6 .8 .2 .5 .14\end{array}$ & 7 REVISION NO & AND AUTHORIZATION & 8 DATE \\
\hline
\end{tabular}

9 APPROVED CHANGES

10 SYSTEM DESIGN DESCRIPTION

11 BUDGET AND REPORTING NUMBER

HO: 93-D-186 (K-003)

EW70300000 / 39EH703000

12 ELEMENT TASK DESCRIPTION

A. COST CONTENT

This project was cancelled in Fy 1994.

B. TECHNICAL CONTENT/WORK STATEMENT 
ICF-0001

\section{LANDLORD PROGRAM •}

FY 1995 MYPP

7.5

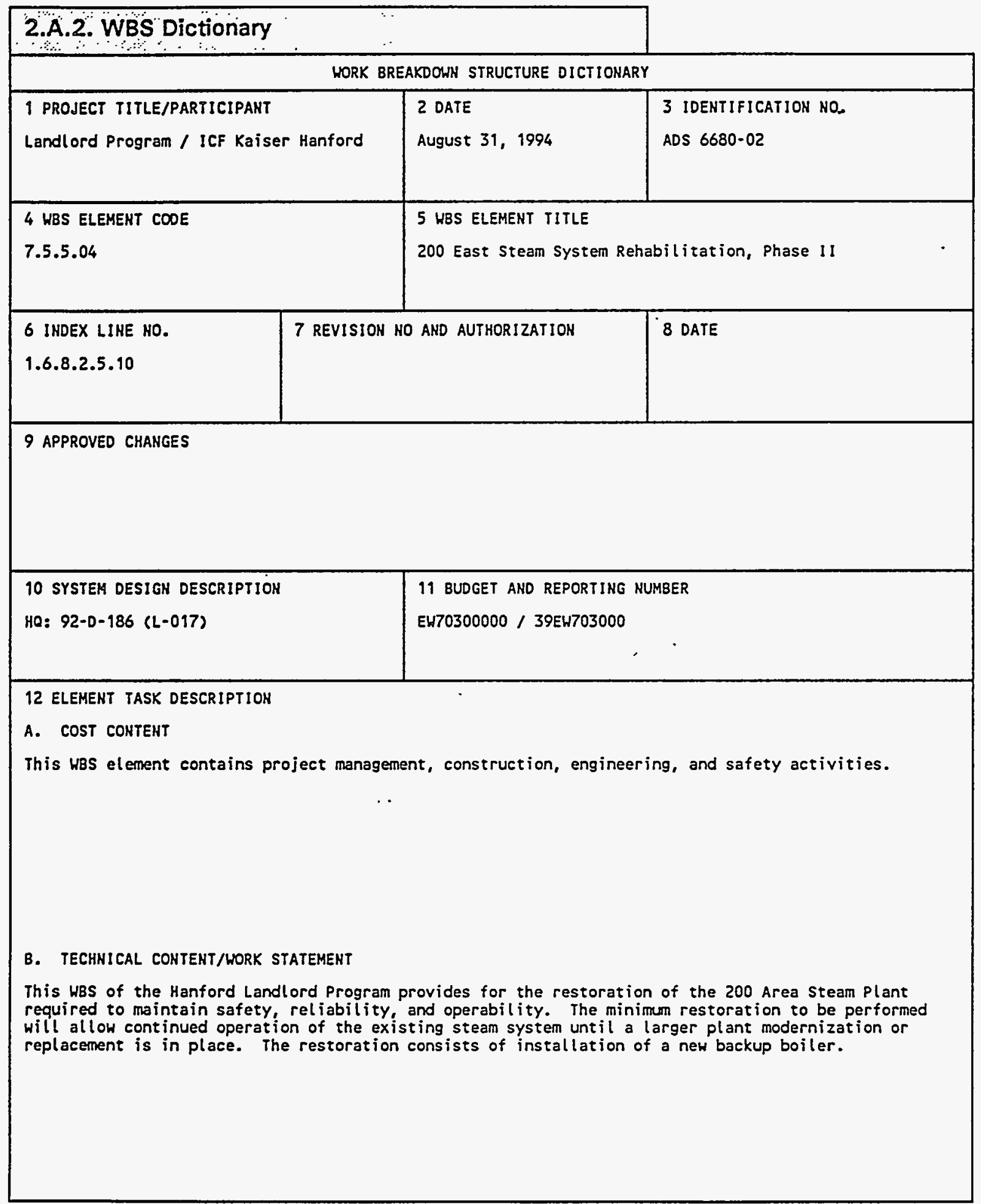




\section{LANDLORD PROGRAM}

FY 1995 MYPP

\section{5}

\begin{tabular}{|c|c|c|c|}
\hline & WORK & AKDOWN STRUCTURE D & \\
\hline $\begin{array}{l}1 \text { PROJECT TITLE/PARTICIPANT } \\
\text { Landlord Program / ICF Kaiser }\end{array}$ & Hanford & $\begin{array}{l}2 \text { DATE } \\
\text { August 31, } 1994\end{array}$ & $\begin{array}{l}3 \text { IDENTIFICATION NO. } \\
\text { ADS } 6680-03\end{array}$ \\
\hline $\begin{array}{l}4 \text { WBS ELEMENT COOE } \\
7.5 .5 .05\end{array}$ & & $\begin{array}{l}5 \text { WBS ELEMENT TI } \\
\text { Landlord Program }\end{array}$ & Ipl iance, Phase I \\
\hline $\begin{array}{l}6 \text { INDEX LINE NO. } \\
1.6 .8 .2 .5 .6\end{array}$ & 7 REVISI & AND AUTHORIZATIC & 8 DATE \\
\hline
\end{tabular}

9 APPROVED CHANGES

10 SYSTEM DESIGN DESCRIPTION

HQ: $90-D-175$
11 BUDGET AND REPORTING NUMBER

EH70300000 / 39EH703000

12 ELEMENT TASK DESCRIPTION

A. COST CONTENT

This UBS element contains program and project management, construction, safety, environmental, miscellaneous surveying and testing, and technical publications activities.

\section{B. TECHNICAL CONTENT/HORK STATEMENT}

This WBS of the Hanford Landlord Program consists of Project B-468, "Railroad Upgrade Mainl ine": Project B-604, "Hater System Upgrades-Reservoir"; Project B-690, "Steam System Safety and Productivity Upgrade"; and Project L-001, "Fire Hater Storage and Distribution Upgrades, 300 Area." Subproject B-468a replaces rail from the Columbia Center to the Yakima River bridge. Subproject B-604 upgrades the capacity of the current water storage and supply systems for the 200 Area to meet RLIP 5480.7 and the National Fire Protection Association standards. Subproject B-690 replaced existing electrical service, circuits and components in the 44 year old steam generation facilities in the 200 Areas and is complete. Subproject L-001 provided upgrades to the existing 300 Area water systems as required to provide an independent water supply capable of meeting fire flow demands simultaneous with peak process and domestic demands for a period of not less than four hours and is complete. 
ICF-000I

\section{LANDLORD PROGRAM}

FY 1995 MYPP

\section{5}

\begin{tabular}{|c|c|c|c|}
\hline \multicolumn{4}{|l|}{ 2:A.2. WBS Dictionary } \\
\hline \multicolumn{4}{|c|}{ WORK BREAKDOWN STRUCTURE DICTIONARY } \\
\hline \multicolumn{2}{|c|}{$\begin{array}{l}1 \text { PROJECT TITLE/PARTICIPANT } \\
\text { Landlord Program / ICF Kaiser Hanford }\end{array}$} & $\begin{array}{l}2 \text { DATE } \\
\text { August } 31,1994\end{array}$ & $\begin{array}{l}3 \text { IDENTIFICATION NO. } \\
\text { ADS } 6680-04\end{array}$ \\
\hline \multicolumn{2}{|l|}{$\begin{array}{l}4 \text { WBS ELEMENT COOE } \\
7.5 .5 .06\end{array}$} & \multicolumn{2}{|c|}{$\begin{array}{l}5 \text { WBS ELEMENT TITLE } \\
300 \text { Area Process Sewer Upgrade Piping System }\end{array}$} \\
\hline $\begin{array}{l}6 \text { IHDEX LIME NO. } \\
1.6 .8 .2 .5 .15\end{array}$ & \multicolumn{2}{|c|}{7 REVISION NO AND AUTHORIZATION } & 8 DATE \\
\hline \multicolumn{4}{|l|}{9 APPROVED CHANGES } \\
\hline \multicolumn{2}{|l|}{$\begin{array}{l}10 \text { SYSTEH DESIGN DESCRIPTION } \\
\text { HO: } 94-D-412 \text { (L-070) }\end{array}$} & \multicolumn{2}{|c|}{$\begin{array}{l}11 \text { BUDGET AND REPORTING NUMBER } \\
\text { EW70300000 / 39EW703000 }\end{array}$} \\
\hline \multicolumn{4}{|c|}{$\begin{array}{l}12 \text { ELEMENT TASK DESCRIPTION } \\
\text { A. COST CONTENT } \\
\text { This WBS element contains program and project management, construction and design engineering, } \\
\text { enviromental support, miscellaneous surveying and testing, and technical publications activities. }\end{array}$} \\
\hline \multicolumn{4}{|c|}{$\begin{array}{l}\text { B. TECHHICAL CONTENT/HORK STATEMENT } \\
\text { This WBS of the Hanford Landlord Program includes all costs specific to a line item project for the } \\
\text { replacement of the } 300 \text { Area process sewer collection system. The existing } 50 \text { year old pipe was used } \\
\text { for many years for unregulated discharge of hazardous and radioactive materials. Limited inspection } \\
\text { indicated the piping is deteriorated and possibly allowed liquids to be released to the ground. It is } \\
\text { planned to replace about } 34,000 \text { lineal feet of suspect vitreous clay pipe with about } 22,000 \text { lineal } \\
\text { feet of smaller diameter high integrity plastic pipe. The new collection network will be a } \\
\text { combination of vacum, gravity and pressurized systems connected to about } 37 \text { buildings. The new piping } \\
\text { nominally starts five feet from connected buildings and discharges to a new collection station } \\
\text { provided by another project in the northern part of the } 300 \text { Area. There are two TPA milestones } \\
\text { associated with the project to 1) submit design documentation by April 1995, and } 2 \text { ) complete } \\
\text { replacement of the piping by June } 1997 \text {. }\end{array}$} \\
\hline
\end{tabular}


ICF-0001

\section{LANDLORD PROGRAM}

FY 1995 MYPP

7.5

\section{A.2. WBS Dictionary}

HORK BREAKDOWH STRUCTURE DICTIONARY

\begin{tabular}{|c|c|c|c|}
\hline $\begin{array}{l}\text { I PROJECT TITLE/PARTICIPANT } \\
\text { Landlord Program / ICF Kaiser }\end{array}$ & Hanford & $\begin{array}{l}2 \text { DATE } \\
\text { August } 31,1994\end{array}$ & $\begin{array}{l}3 \text { IDENTIFICATION NO. } \\
\text { ADS } 6680-05\end{array}$ \\
\hline $\begin{array}{l}4 \text { UBS ELEMENT CODE } \\
7.5 .5 .07\end{array}$ & & $\begin{array}{l}5 \text { WBS ELEMENT TITLE } \\
\text { Hanford Infrastructur }\end{array}$ & derground Storage Tanks \\
\hline $\begin{array}{l}6 \text { IHDEX LINE NO. } \\
\text { 1.6.8.2.5.8 }\end{array}$ & 7 REVISION & AND AUTHORIZATION & 8 DATE \\
\hline
\end{tabular}

9 APPROVED CHANGES

10 SYSTEM DESIGN DESCRIPTION

11 BUDGET AND REPORTING NUMBER

HQ: $92-D-184(L-044)$

EW70300000 / 39EW703000

12 ELEMENT TASK DESCRIPTION

A. COST CONTENT

This UBS element contains project management, engineering, construction, and safety activities.

\section{B. TECHKICAL CONTENT/HORK STATEMENT}

This HBS of the Hanford Landlord Program consists of the Underground Storage Tank (UST) project provides the necessary capital funds to upgrade and/or remove petroleum tanks on the Hanford Site to conform with recently enacted Environmental Protection Agency (EPA) and Washington State regulations. Consolidation of three fuelling facilities into one 200 East station will also be included within the scope of this project. Approximately 23 tanks are to be removed in this project and 19 tanks will be replaced. 
ICF-0001

\section{LANDLORD PROGRAM}

FY 1995 MYPP

\section{5}

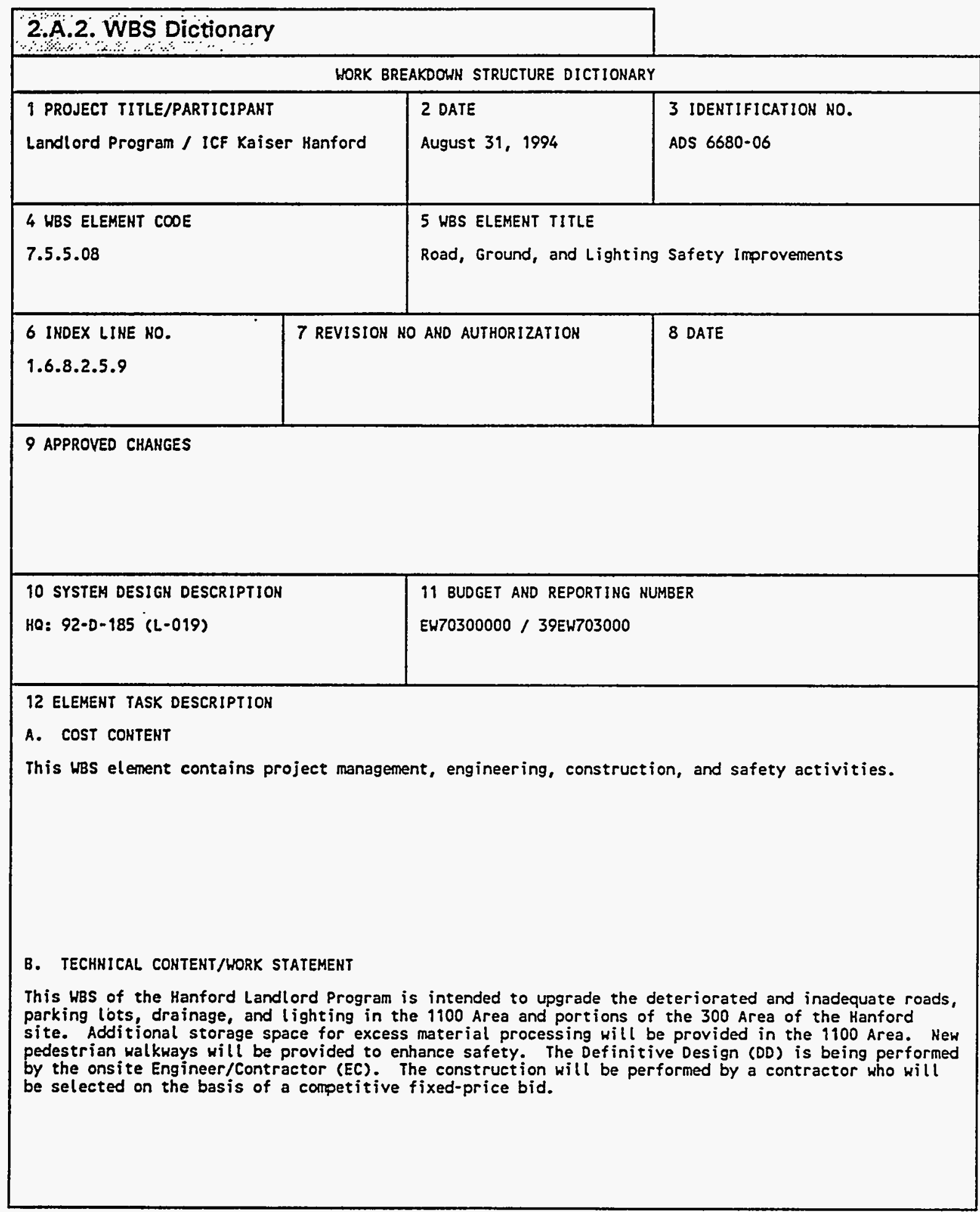

\section{A.2-11}


ICF-0001

\section{LANDLORD PROGRAM}

FY 1995 MYPP

\section{5}

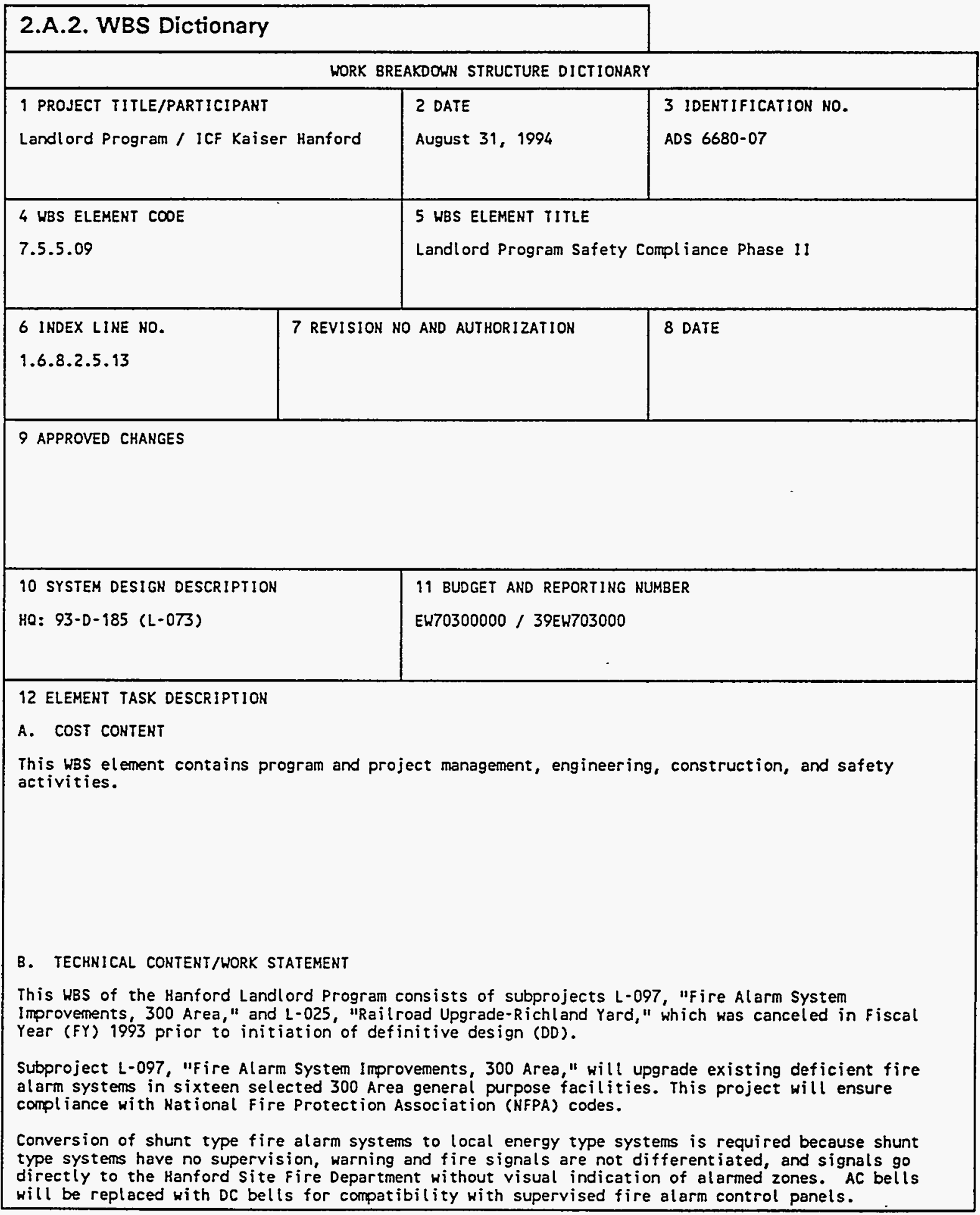


ICF-0001

FY 1995 MYPP

LANDLORD PROGRAM

7.5

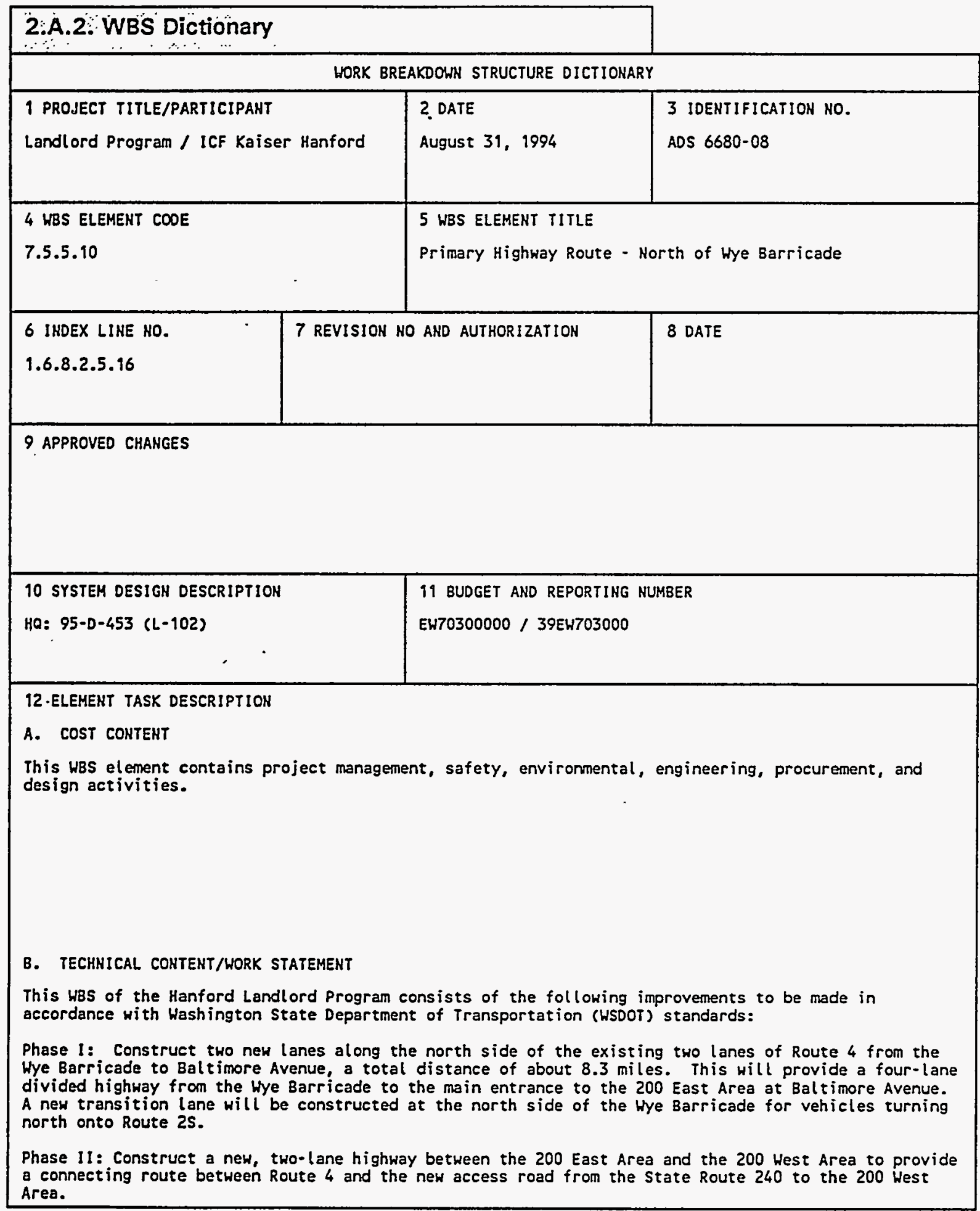




\section{LANDLORD PROGRAM}

FY 1995 MYPP

\section{5}

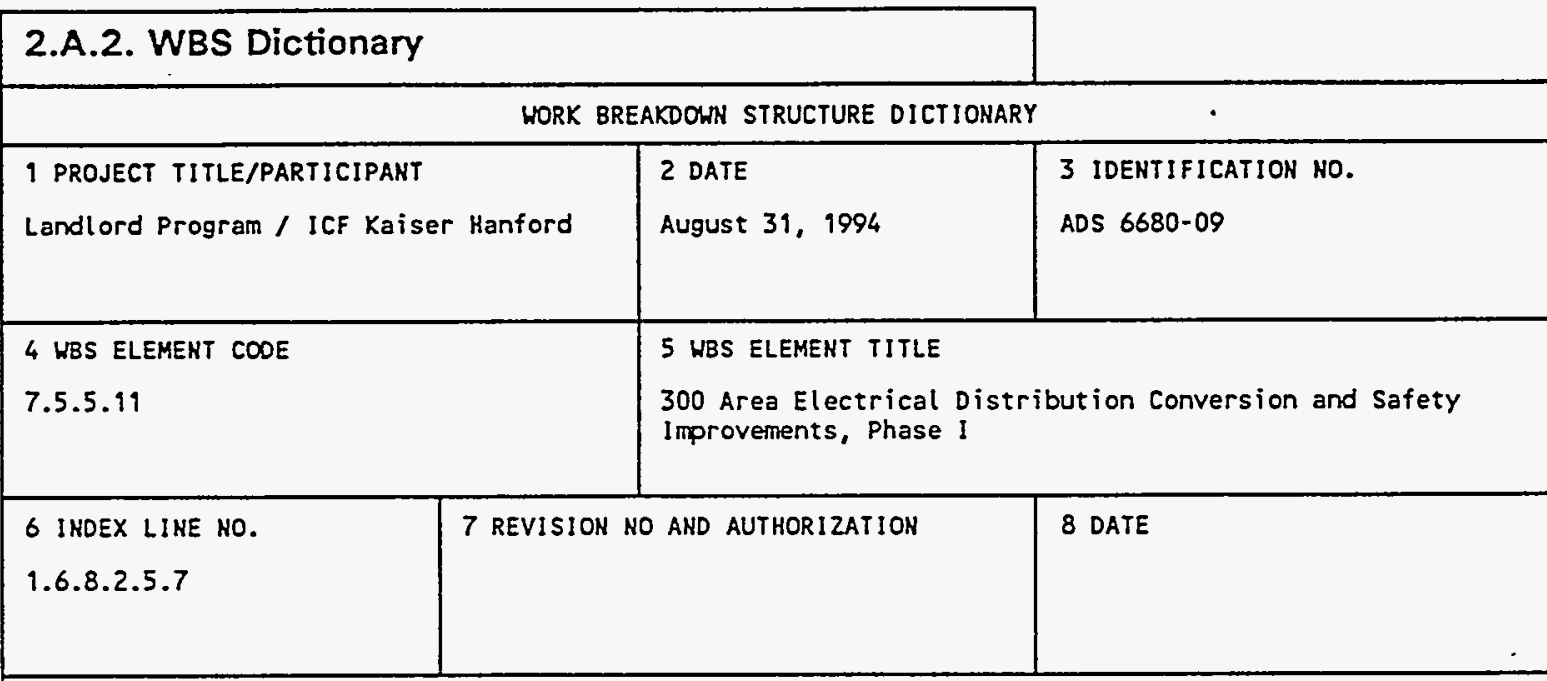

9 APPROVED CHANGES

10 SYSTEM DESIGH DESCRIPTION

KQ: $91-0-175(B-524)$

11 BUDGET AND REPORTING NUMBER

EW70300000 / 39EW703000

12 ELEMENT TASK DESCRIPTION

A. COST CONTENT

This WBS element contains program and project management, safety, environmental, engineering, and construetion activity.

\section{B. TECHHICAL CONTENT/HORK STATEMENT}

This WBS of the Hanford Landlord Program will convert the outdated 2,400 volt electrical distribution circuits in the southern 300 Area to modern 13,800 volt circuits and place the distribution circuits underground. The project is required to provide an electrical distribution system for the southern 300 Area which is safe, efficient, reliable, and maintainable. The project will also improve the lighting for the areas converted. The modernized electrical distribution and lighting systems will provide greater reliability, allow for expansion as necessary, and provide more efficient lighting. This project will be conducted to support environmental restoration mission needs, as well as, meeting the lighting reduction requirements for the new site security initiative (Vision 2000) and energy conservation. New 13,800 volt power sources with associated equipment and new low voltage, parallel connected, lighting systems with high efficiency light fixtures will be provided. 


\section{LANDLORD PROGRAM}

\section{5}

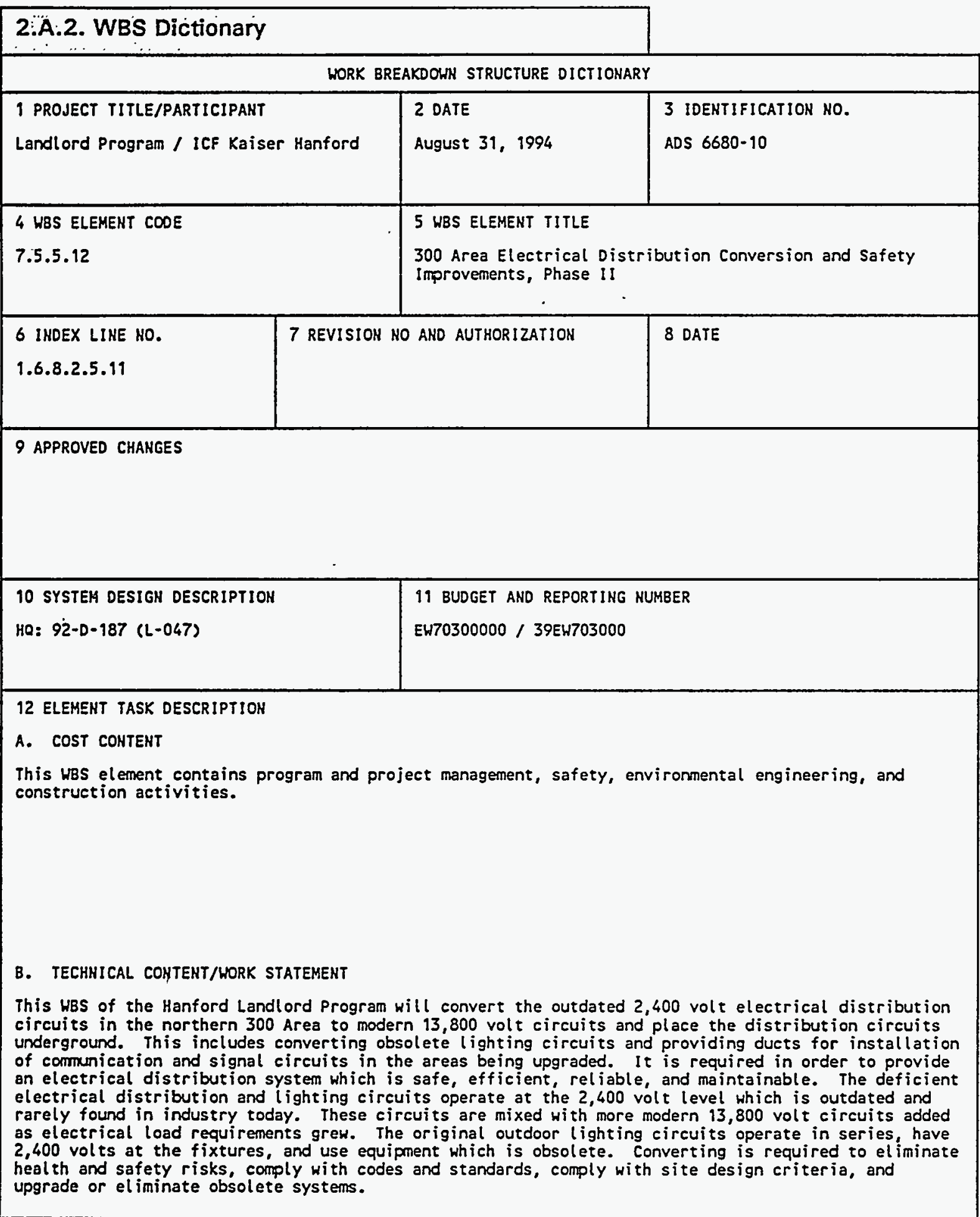


ICF-0001

\section{LANDLORD PROGRAM}

FY 1995 MYPP

7.5

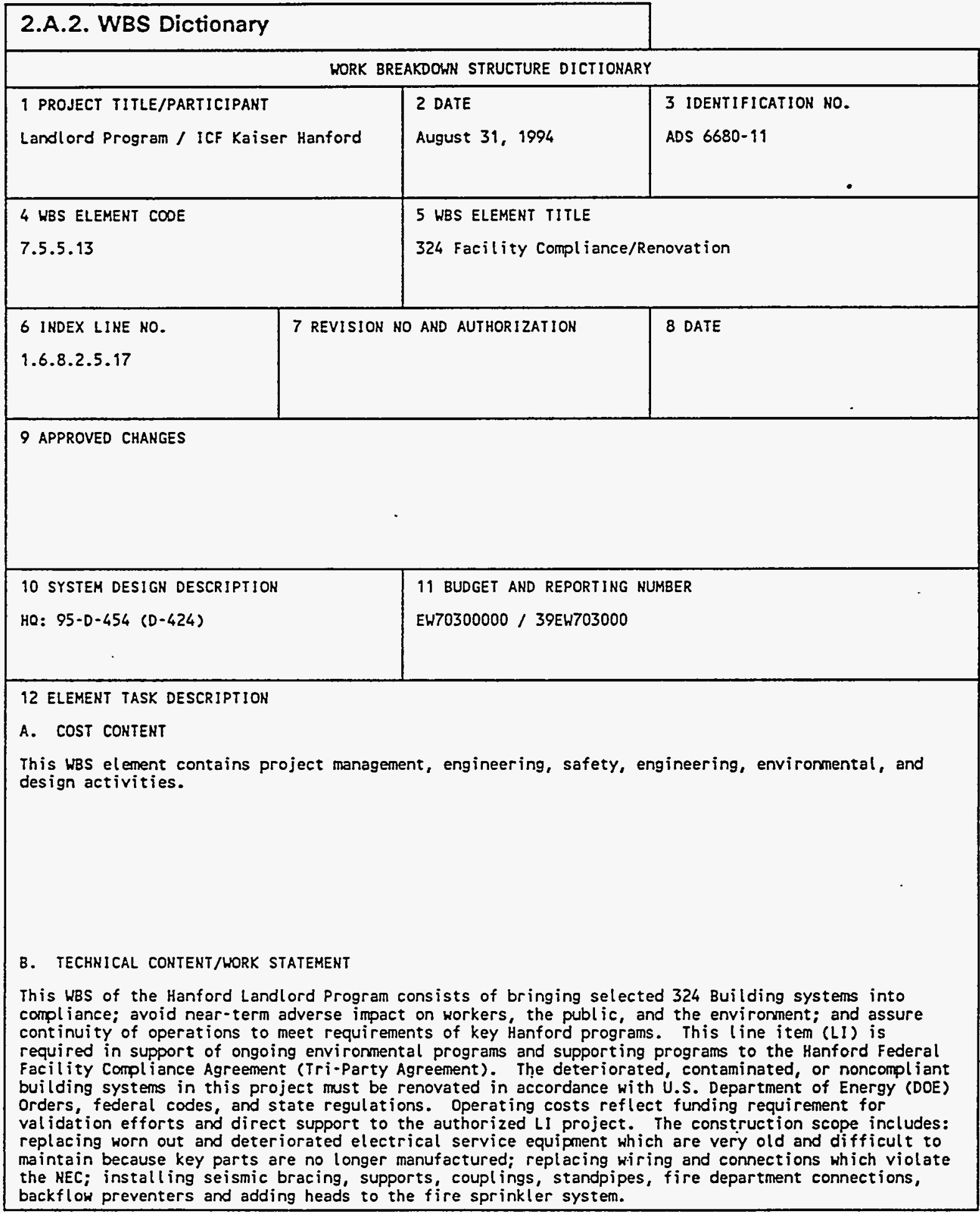




\section{LANDLORD PROGRAM}

FY 1995 MYPP

$$
7.5
$$

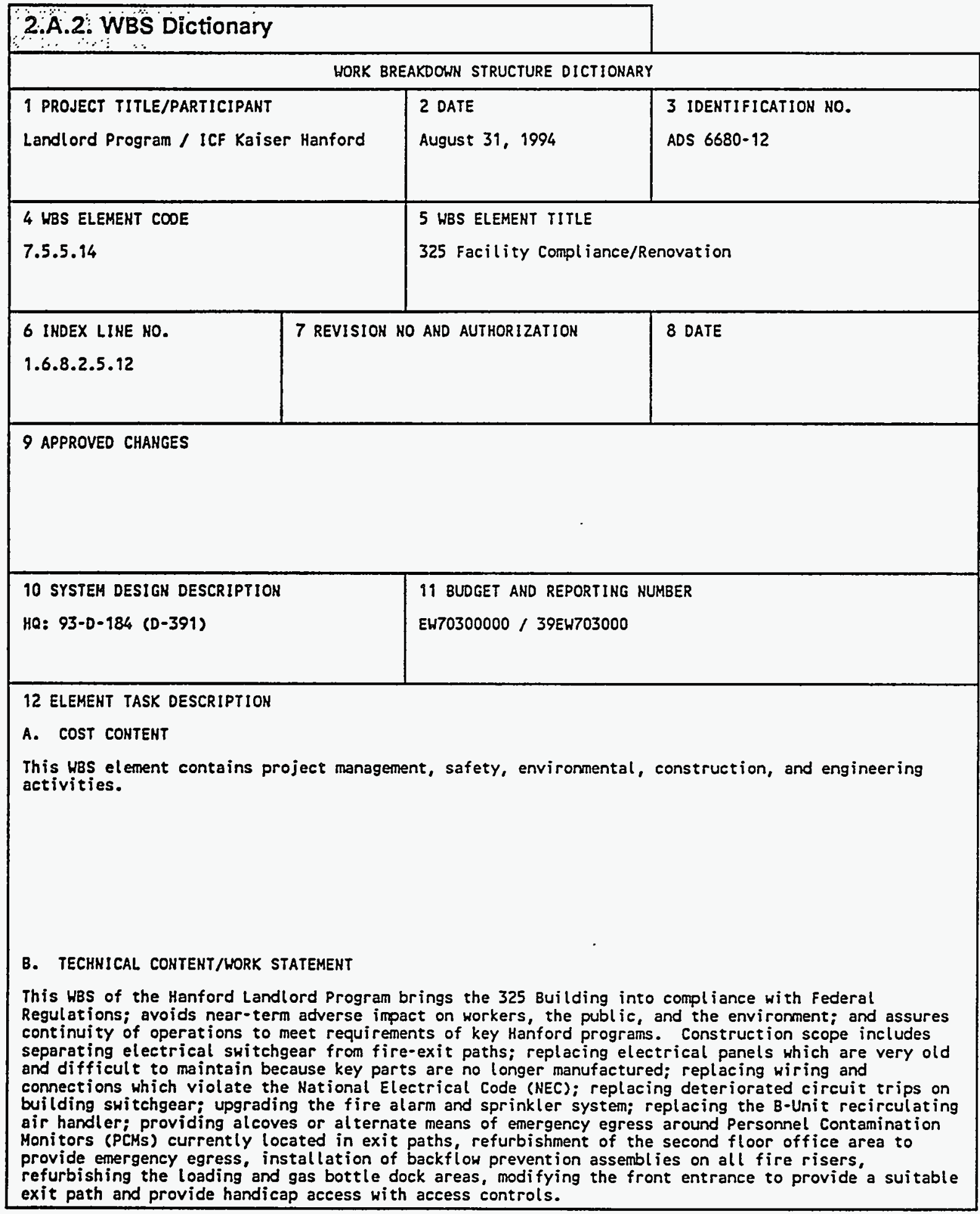


ICF-0001

\section{LANDLORD PROGRAM}

FY 1995 MYPP

\section{5}

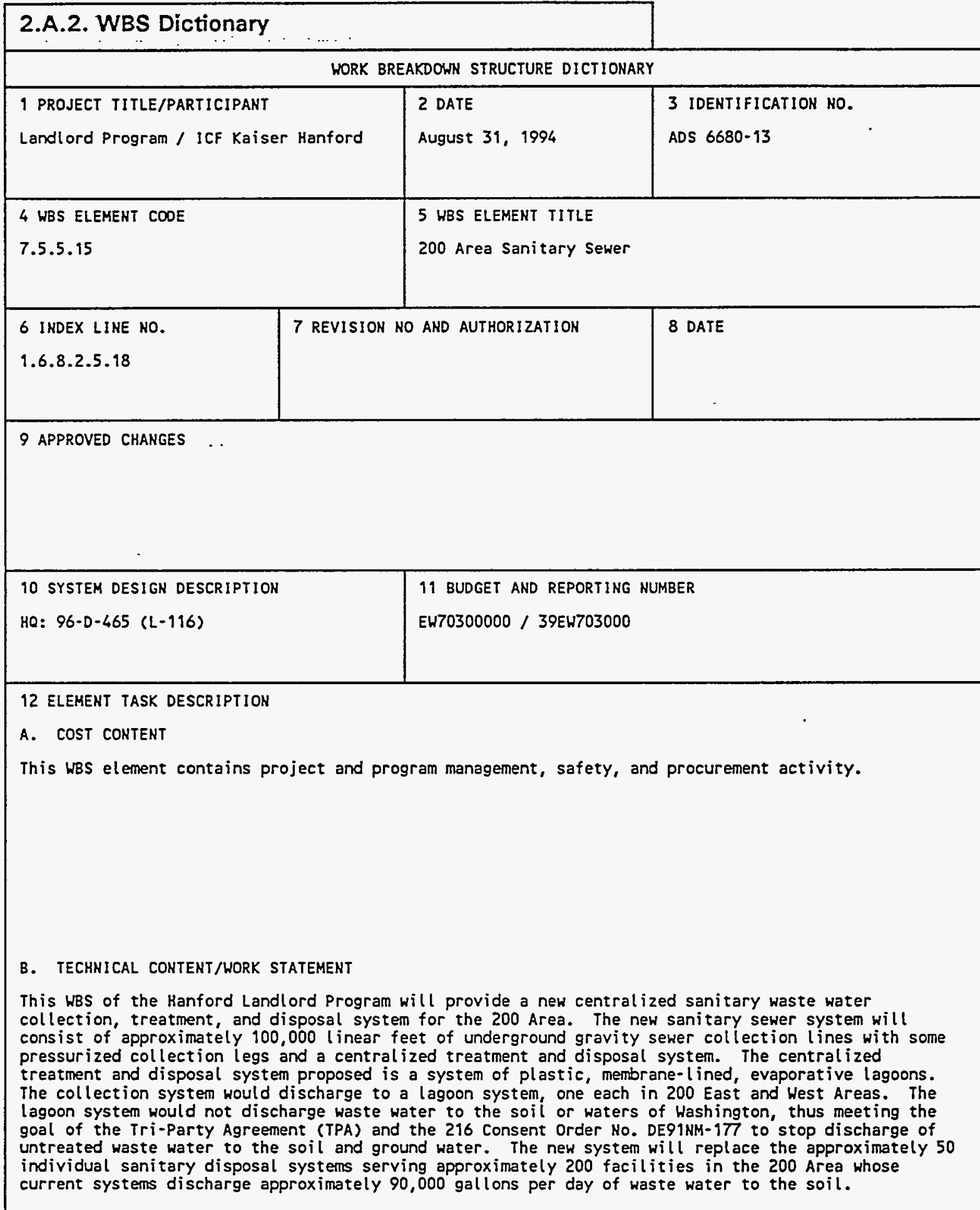




\section{LANDLORD PROGRAM}

\section{5}

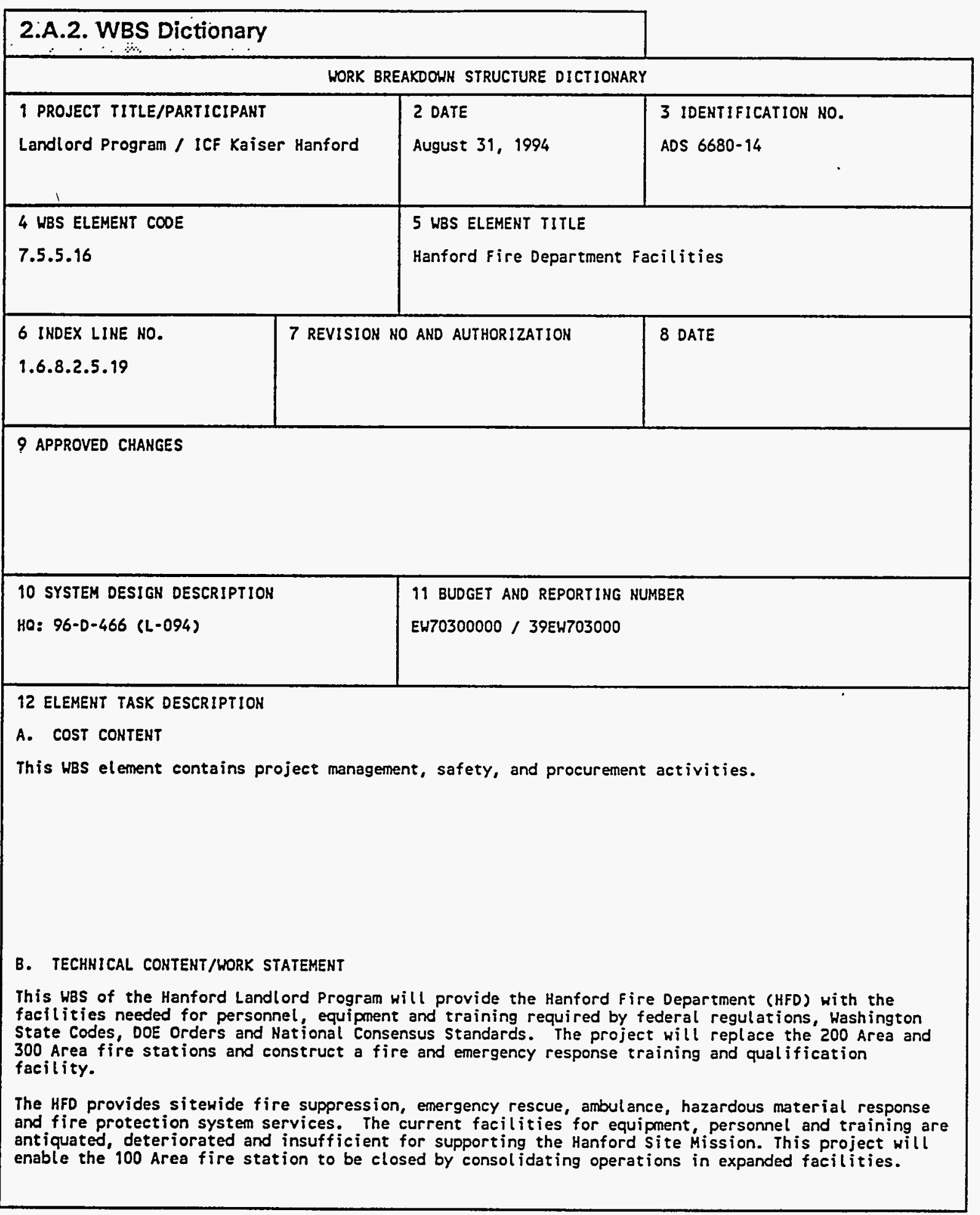

\section{A.2-19}


ICF-0001

\section{LANDLORD PROGRAM}

FY 1995 MYPP

7.5

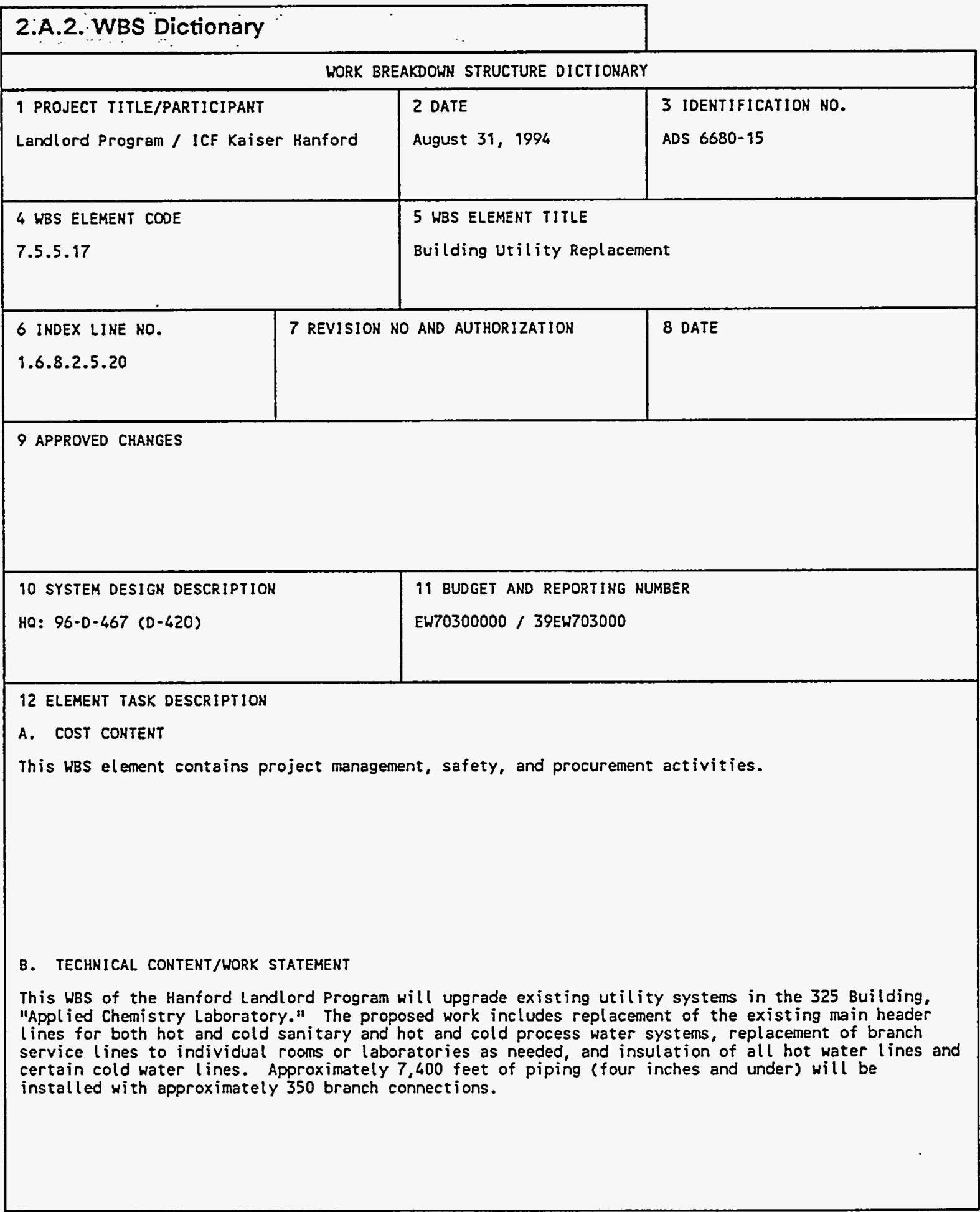




\section{LANDLORD PROGRAM}

FY 1995 MYPP

7.5

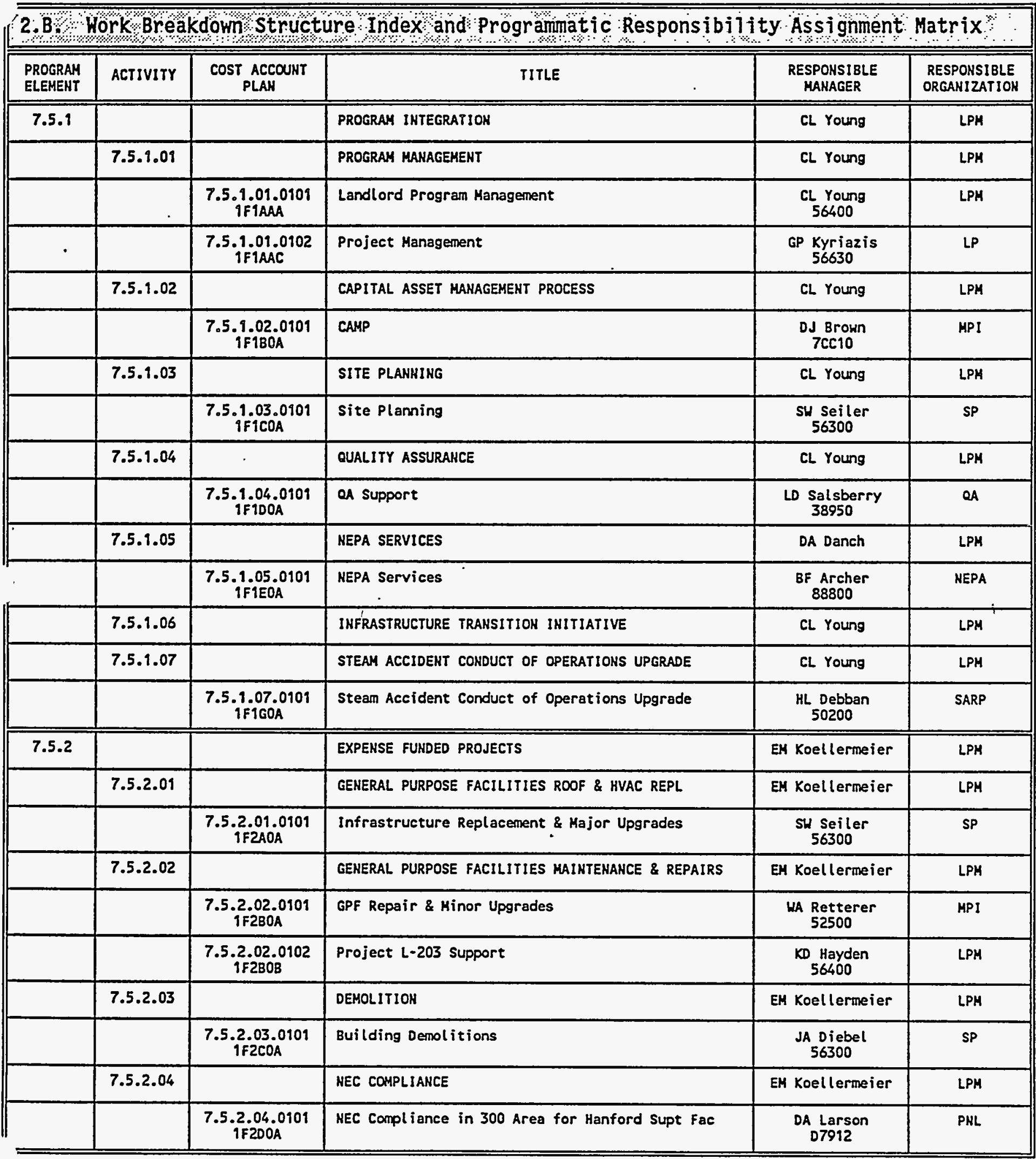


ICF-000I

\section{LANDLORD PROGRAM}

FY 1995 MYPP

\section{5}

\section{B. Work Breakdown Structure Index and Programatic Responsibjlity Ass ignment Matrix}

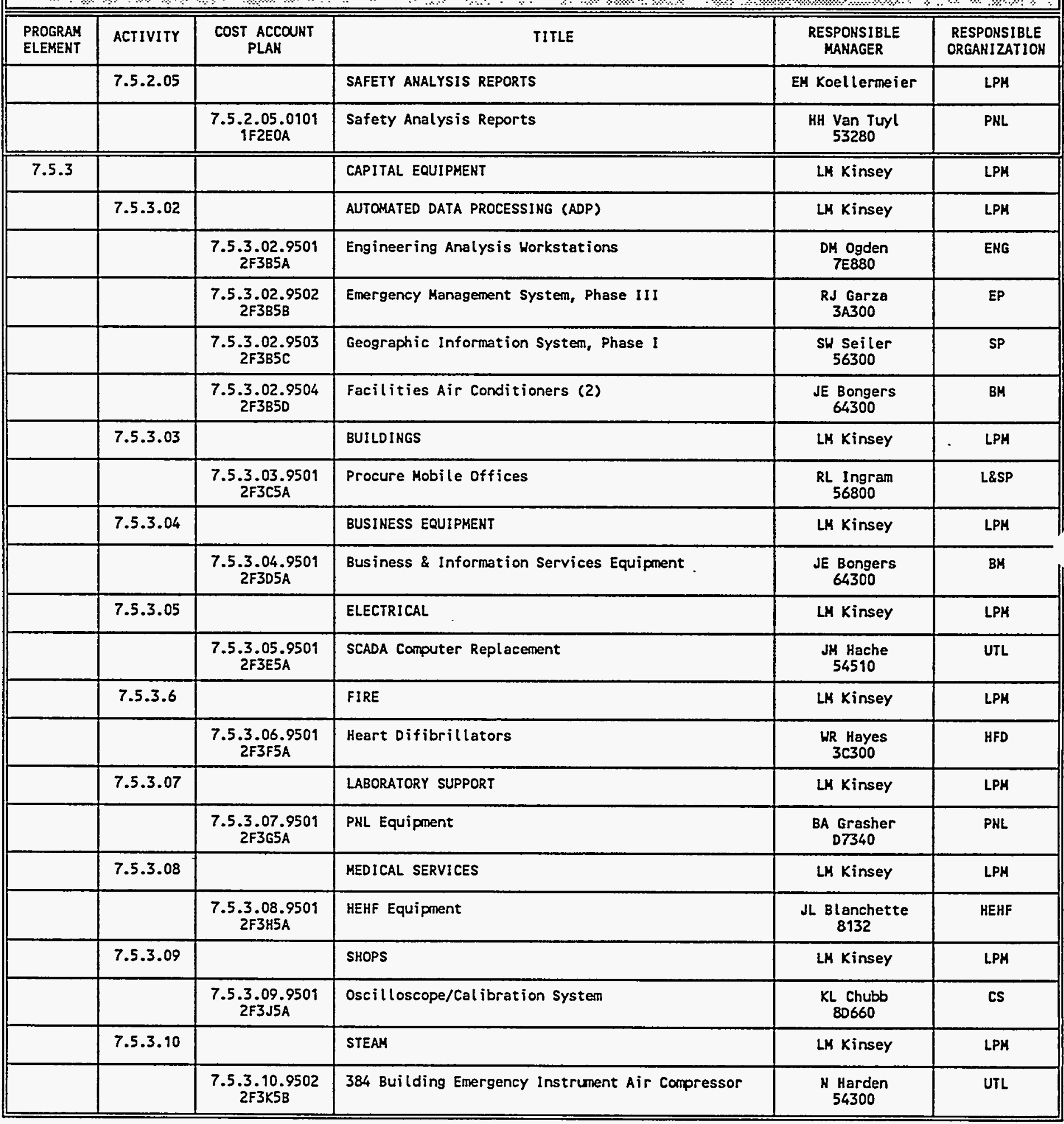




\section{LANDLORD PROGRAM}

FY 1995 MYPP

\section{5}

\begin{tabular}{|c|c|c|c|c|c|}
\hline $\begin{array}{l}\text { PROGRAM } \\
\text { ELEMENT }\end{array}$ & ACTIVITY & $\begin{array}{c}\text { COST ACCOUNT } \\
\text { PLAN }\end{array}$ & TITLE & $\begin{array}{l}\text { RESPONSIBLE } \\
\text { MANAGER }\end{array}$ & $\begin{array}{l}\text { RESPONSIBLE } \\
\text { ORGANIZATION }\end{array}$ \\
\hline & & $\begin{array}{l}7.5 .3 .10 .9503 \\
2 F 3 K 5 C\end{array}$ & $284 E$ Water Softeners & $\begin{array}{l}\text { DE Maki } \\
54200\end{array}$ & UTL \\
\hline & & $\begin{array}{c}7.5 .3 .10 .9504 \\
2 F 3 \times 50\end{array}$ & 384 Building Condensate Return System & $\begin{array}{l}\text { H Harden } \\
54300\end{array}$ & UTL \\
\hline & 7.5.3.11 & & TELECOHAUNICATIONS & LM Kinsey & LPH \\
\hline & & $\begin{array}{c}\text { 7.5.3.11.9501 } \\
2 \mathrm{~F} 3 \mathrm{LAA}\end{array}$ & Communications Replacement Equipment & $\begin{array}{c}\text { AD Ball linger } \\
69600\end{array}$ & TEL \\
\hline & & $\begin{array}{c}7.5 .3 .11 .9502 \\
2 F 3 L 58\end{array}$ & Communications Test Equipment & $\begin{array}{c}\text { AD Ball inger } \\
69600\end{array}$ & TEL \\
\hline & 7.5 .3 .12 & & TRANSPORTATIOH & LH Kinsey & LPH \\
\hline & & $\begin{array}{c}7.5 .3 .12 .9501 \\
2 F 3 H 5 A\end{array}$ & Transportation & $\begin{array}{l}\text { GL Higgins } \\
55000\end{array}$ & TFS \\
\hline & 7.5 .3 .13 & & HATER/SEWER & LH Kinsey & LPM \\
\hline$\cdot m$ & & $\begin{array}{c}7.5 .3 .13 .9501 \\
2 F 3 \text { NSB }\end{array}$ & 1828/D Monorail Upgrade & $\begin{array}{l}\text { LH Garrelts } \\
54600\end{array}$ & UTL \\
\hline$\because$ & 7.5.3.14 & & IHFORMATIOH/BUSINESS SERVICES & LH Kinsey & LPH \\
\hline \multirow[t]{14}{*}{$7: 5.4$} & & & GENERAL PLANT PROJECTS & EA Koellermeier & LPH \\
\hline & 7.5.4.01 & & PROJECT IDENTIFICATION \& SCOPING & EH Koellermeier & LPH \\
\hline & & $\begin{array}{c}7.5 .4 .01 .0001 \\
1 F 410 \mathrm{~A}\end{array}$ & GPP ID \& Scoping & $\begin{array}{l}\text { DA Danch } \\
56400\end{array}$ & LPH \\
\hline & 7.5 .4 .02 & & GENERAL PLANT PROJECTS/CAPITAL WORK ORDERS & EH Koellermeier & LPH \\
\hline & & $\begin{array}{c}7.5 .4 .02 .0101 \\
\text { IF } 42 \mathrm{CW}\end{array}$ & Small Projects Management & $\begin{array}{c}\text { EM Koell ermeier } \\
56400\end{array}$ & LPH \\
\hline & & $\begin{array}{c}7.5 .4 .02 .9302 \\
4 F 423 \mathrm{~B}\end{array}$ & 93-L-109 384 Building Control Room Expansion & $\begin{array}{c}\text { MV Scott } \\
56630\end{array}$ & LP \\
\hline & & $\begin{array}{c}7.5 .4 .02 .9313 \\
4 F 423 \mathrm{H}\end{array}$ & 93-0-431 324 office Addition & $\begin{array}{c}\text { Vu Briggs } \\
\text { D7912 }\end{array}$ & PNL \\
\hline & & $\begin{array}{c}7.5 .4 .02 .9401 \\
4 F 424 \mathrm{~A}\end{array}$ & 94-L-119 384 Powerhouse Upgrades & $\begin{array}{c}\text { MV Scott } \\
56630\end{array}$ & LP \\
\hline & & $\begin{array}{c}7.5 .4 .02 .9404 \\
4 F 4240\end{array}$ & 94-D-439318 office Addition & $\begin{array}{l}\text { WH Briggs } \\
\text { D7912 }\end{array}$ & PNL \\
\hline & & $\begin{array}{c}7.5 .4 .02 .9500 \\
\text { IF } 420 E\end{array}$ & GPP Construction Support & $\begin{array}{c}\text { EM Koel lermeier } \\
56400\end{array}$ & LPM \\
\hline & & $\begin{array}{c}7.5 .4 .02 .9501 \\
4 F 425 A\end{array}$ & 95-L-221 200 Area Interior Roads & $\begin{array}{l}\text { GP Kyriazis } \\
56630\end{array}$ & LP \\
\hline & & $\begin{array}{c}7.5 .4 .02 .9503 \\
4 F 425 \mathrm{C}\end{array}$ & 95-L-234 400 Area Sanitary Lagoon & $\begin{array}{l}\text { LA Bast } \\
56631\end{array}$ & LP \\
\hline & & $\begin{array}{c}7.5 .4 .02 .9504 \\
4 F 4250\end{array}$ & 95-0-446 324 High Bay Addition & $\begin{array}{c}\text { EM Koell lermeier } \\
56400\end{array}$ & LPM \\
\hline & & $\begin{array}{c}7.5 .4 .02 .9505 \\
4 F 425 E\end{array}$ & 95-D-453 Fire Protection Compliance & $\begin{array}{l}\text { EM Koell lermeier } \\
56400\end{array}$ & LPM \\
\hline
\end{tabular}


FY 1995 MYPP

\section{LANDLORD PROGRAM}

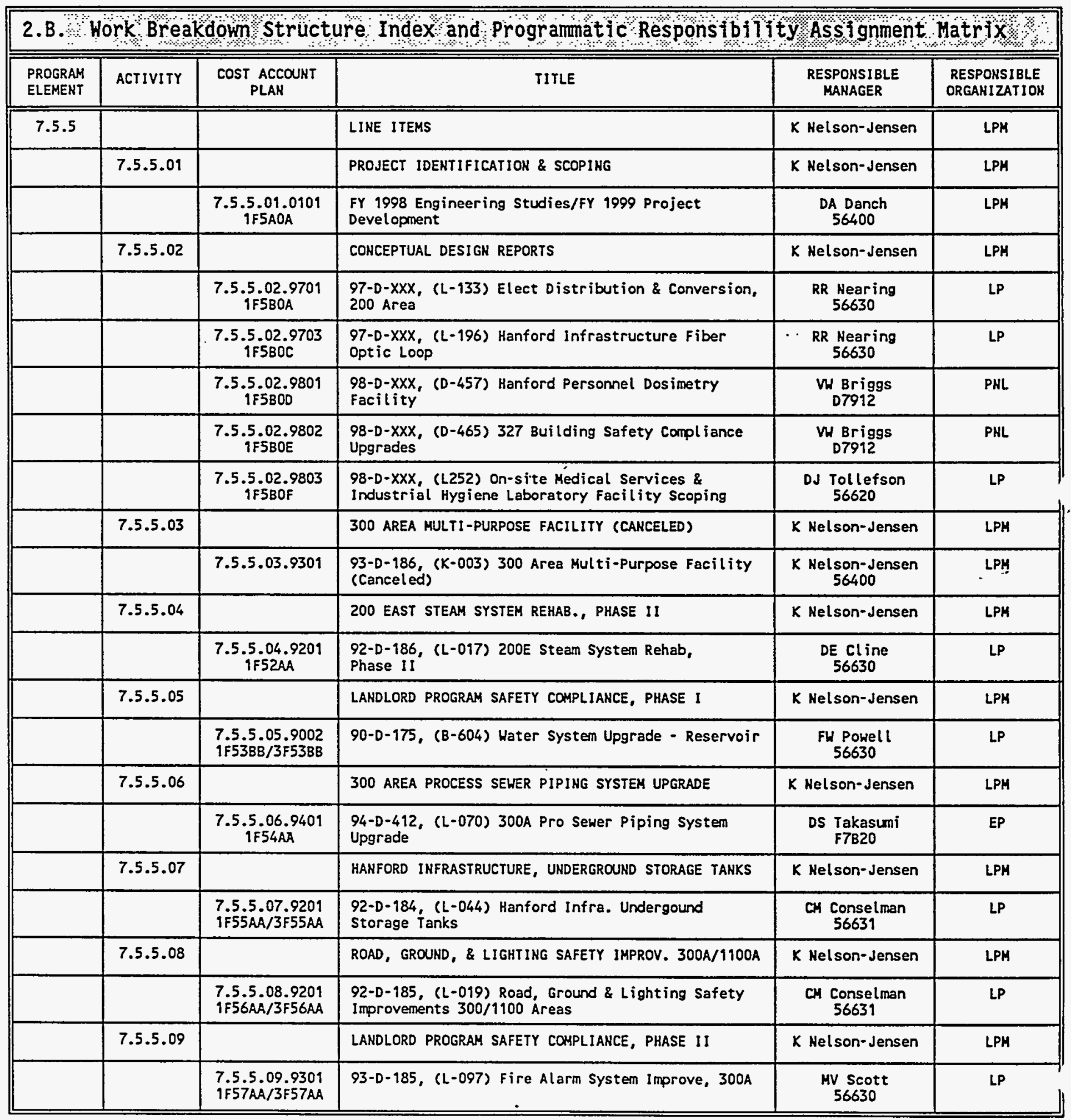


ICF-0001

\section{LANDLORD PROGRAM}

FY 1995 MYPP

7.5

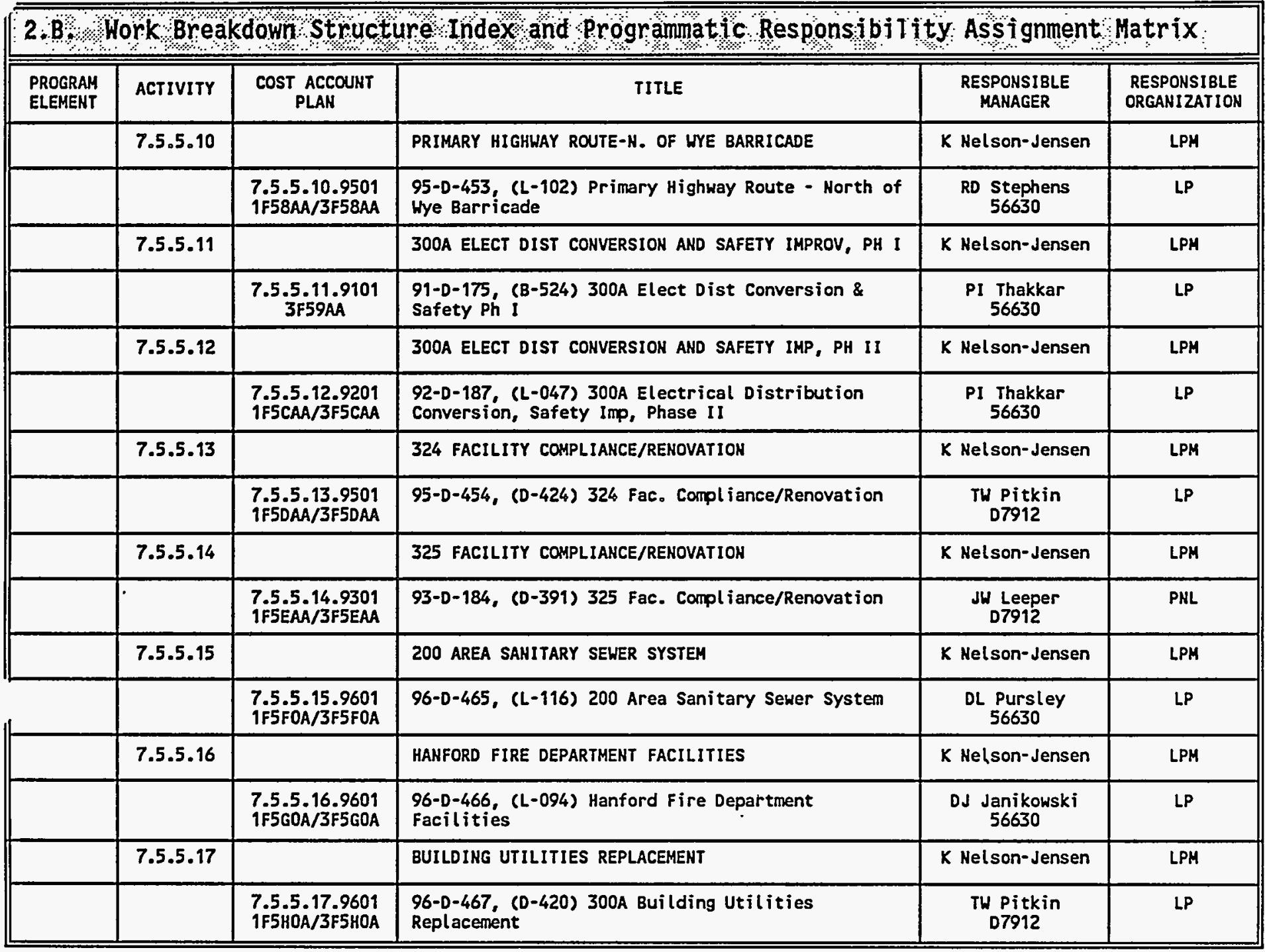




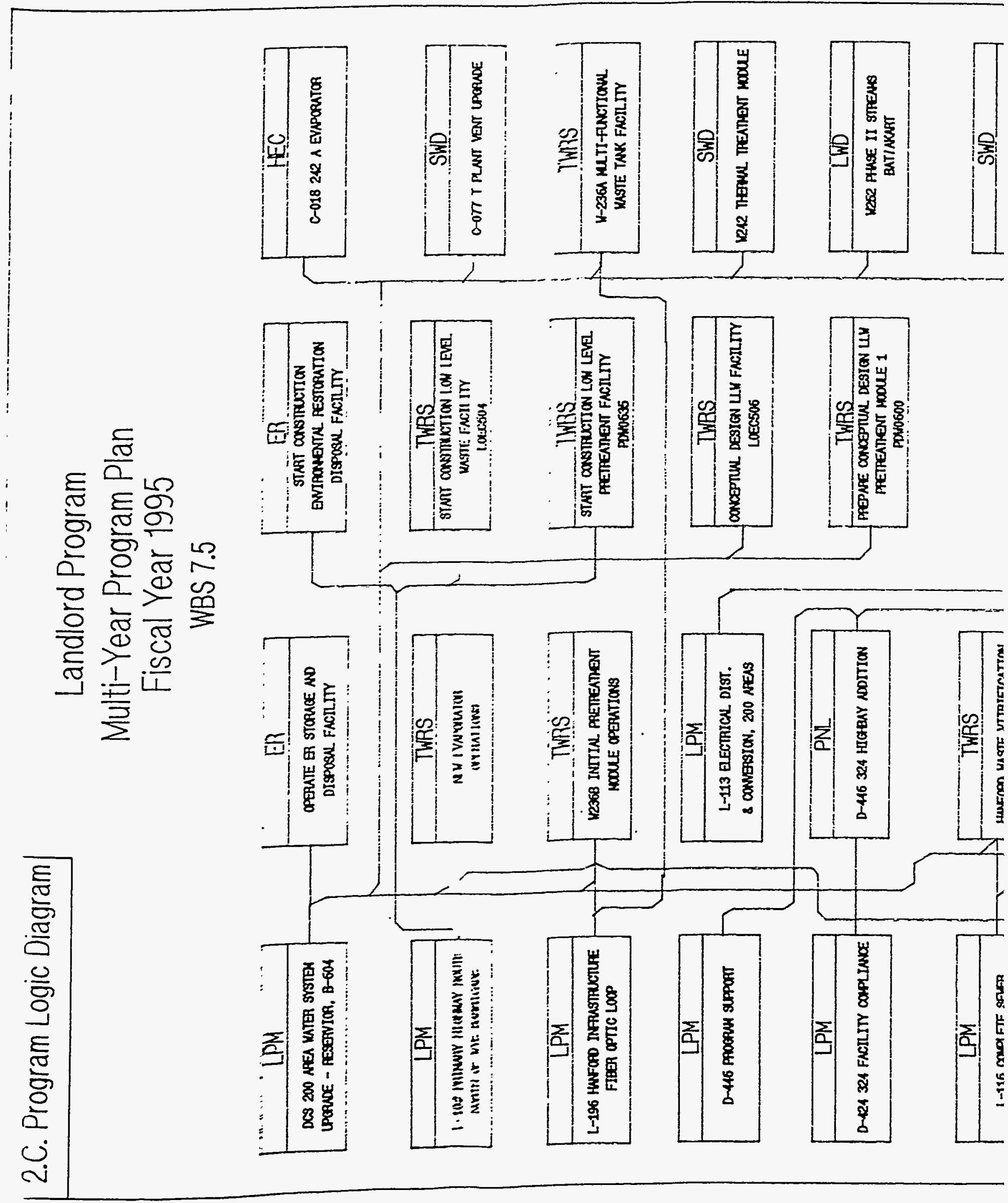



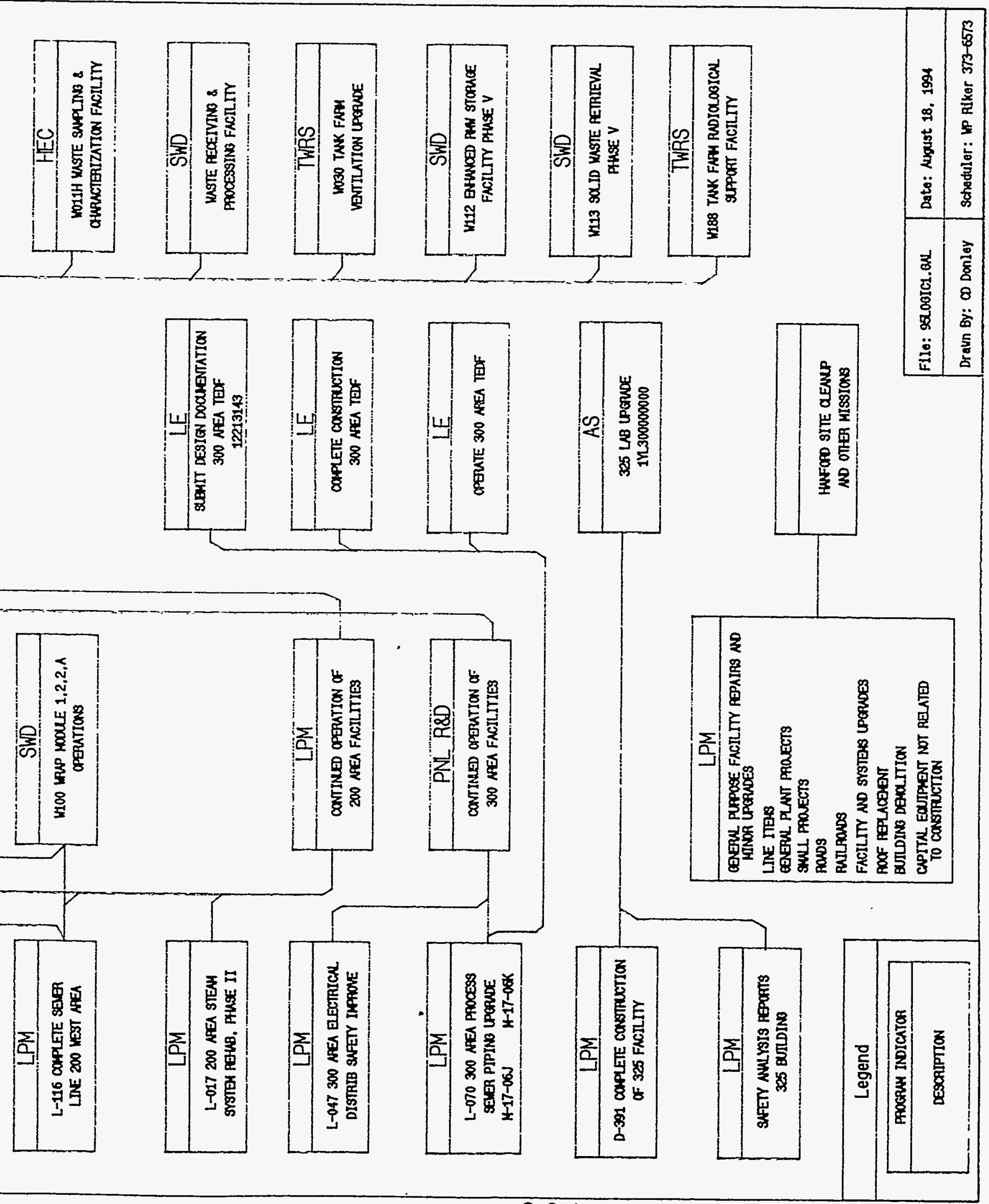


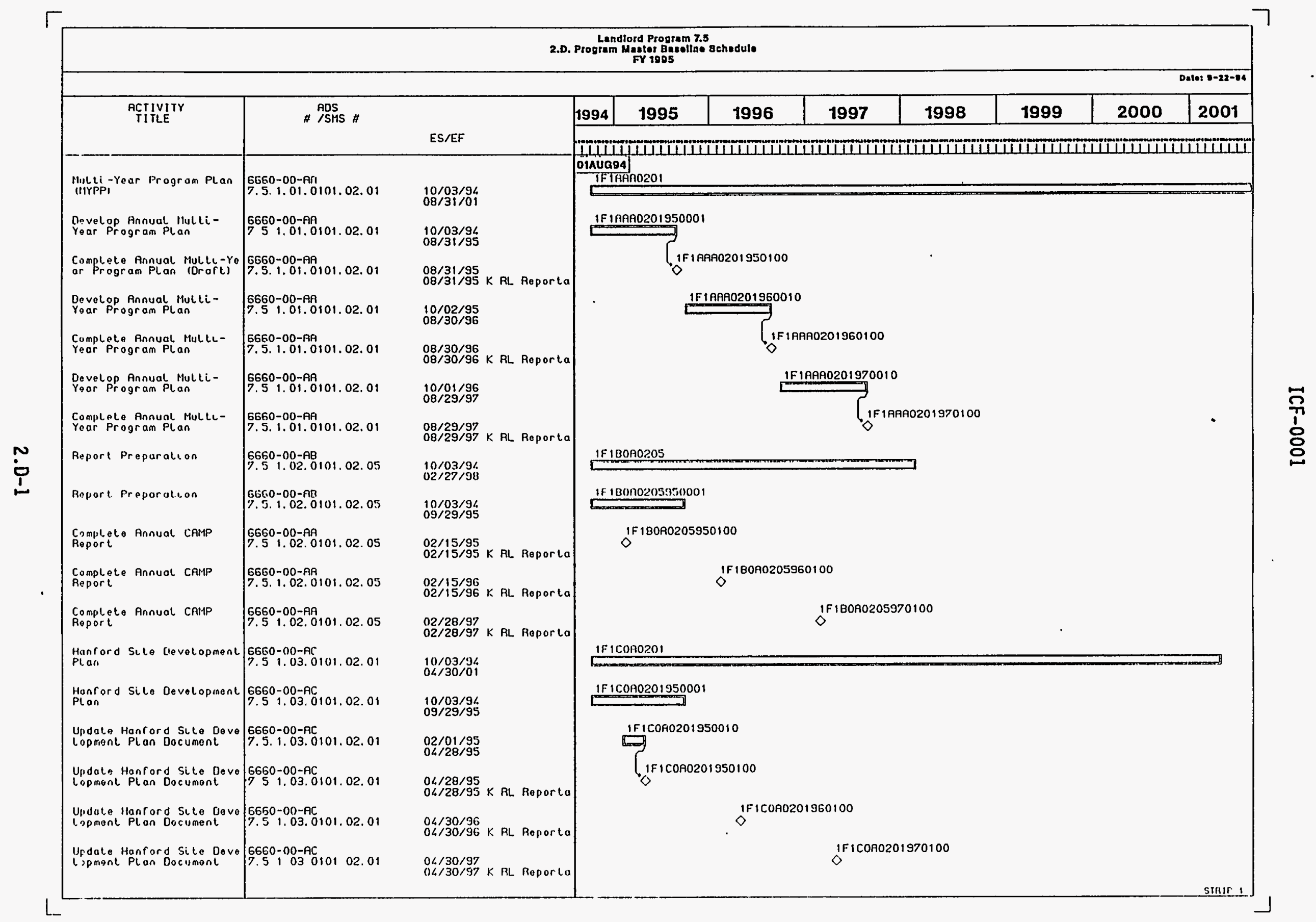




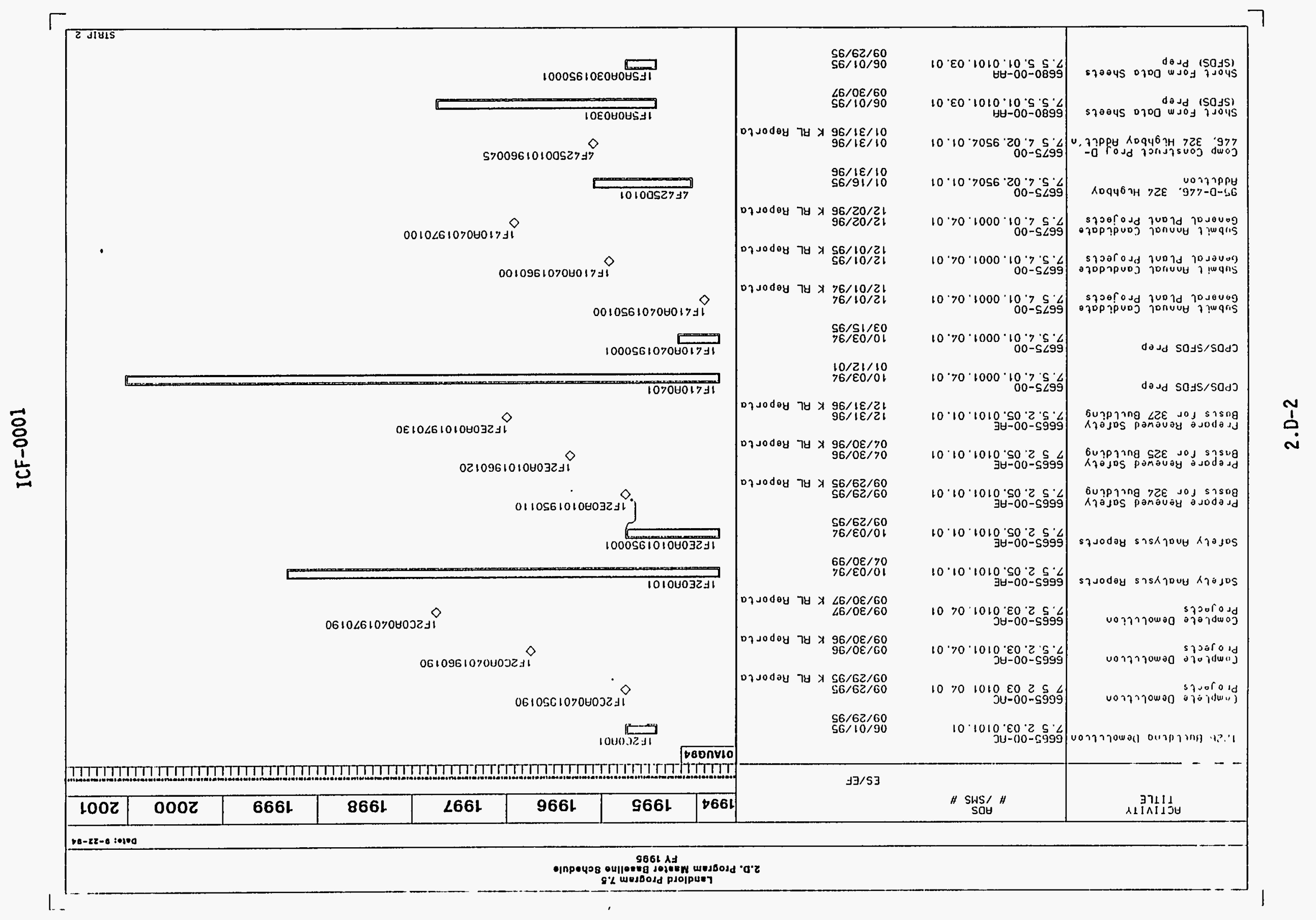




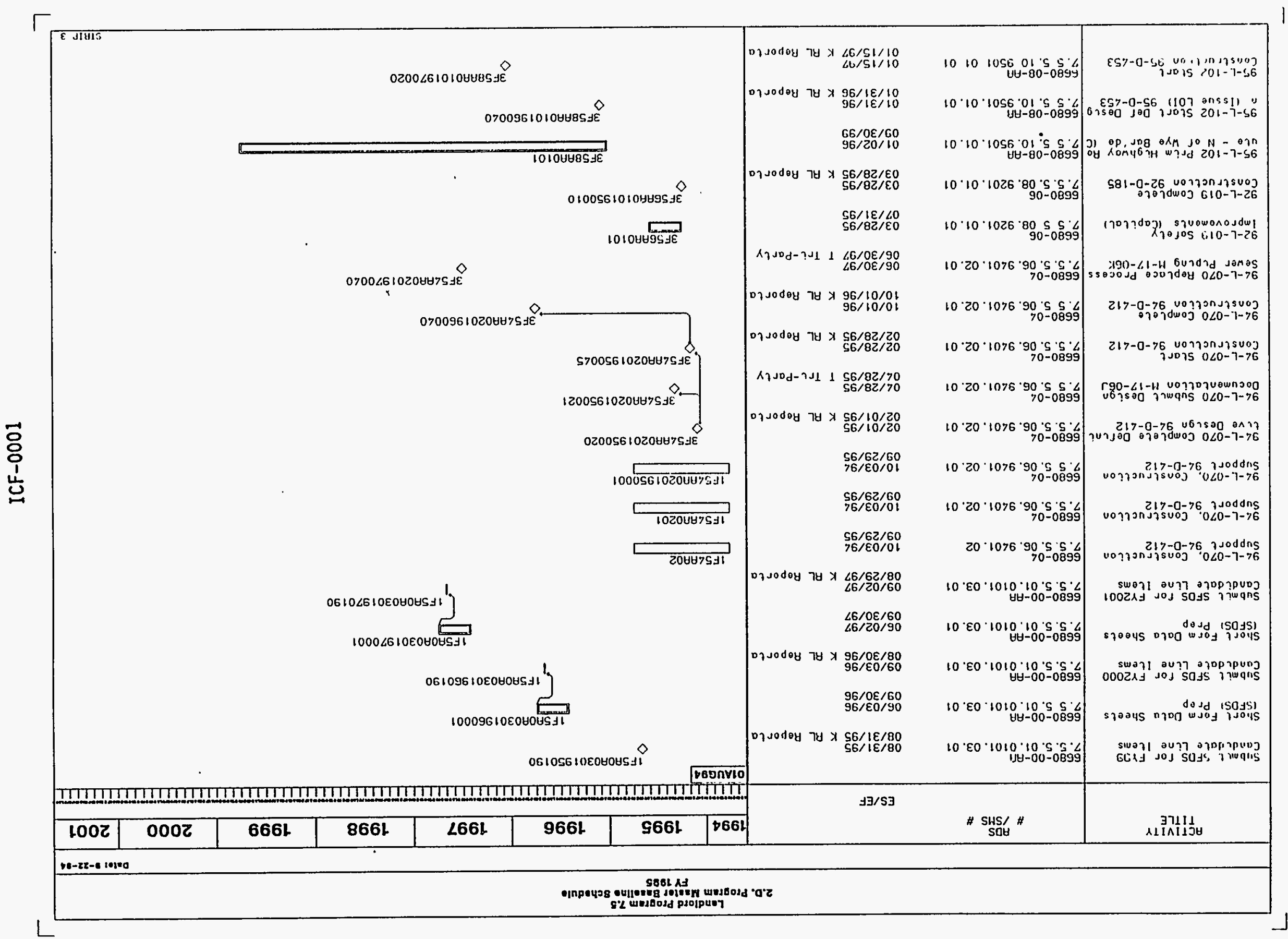




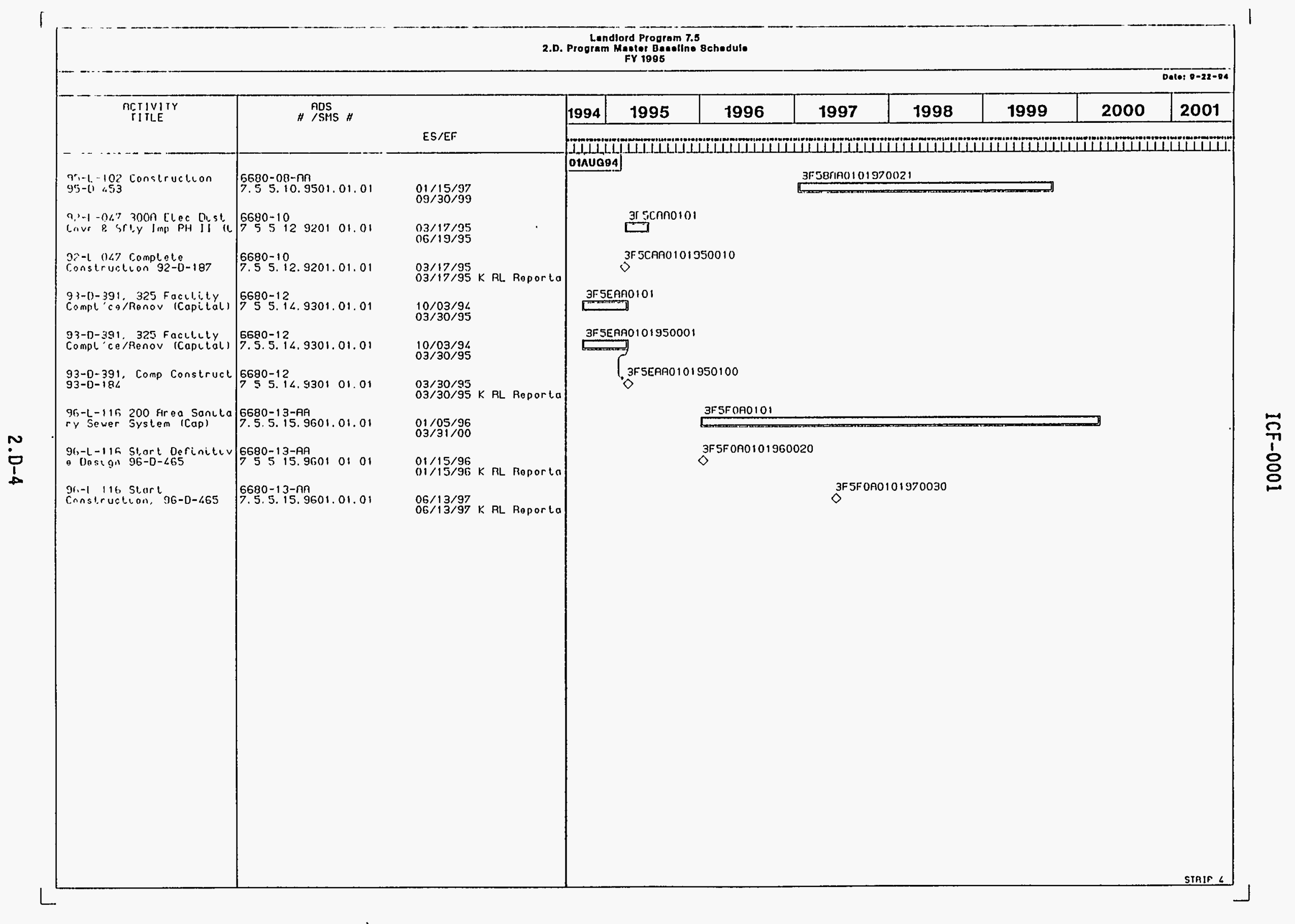




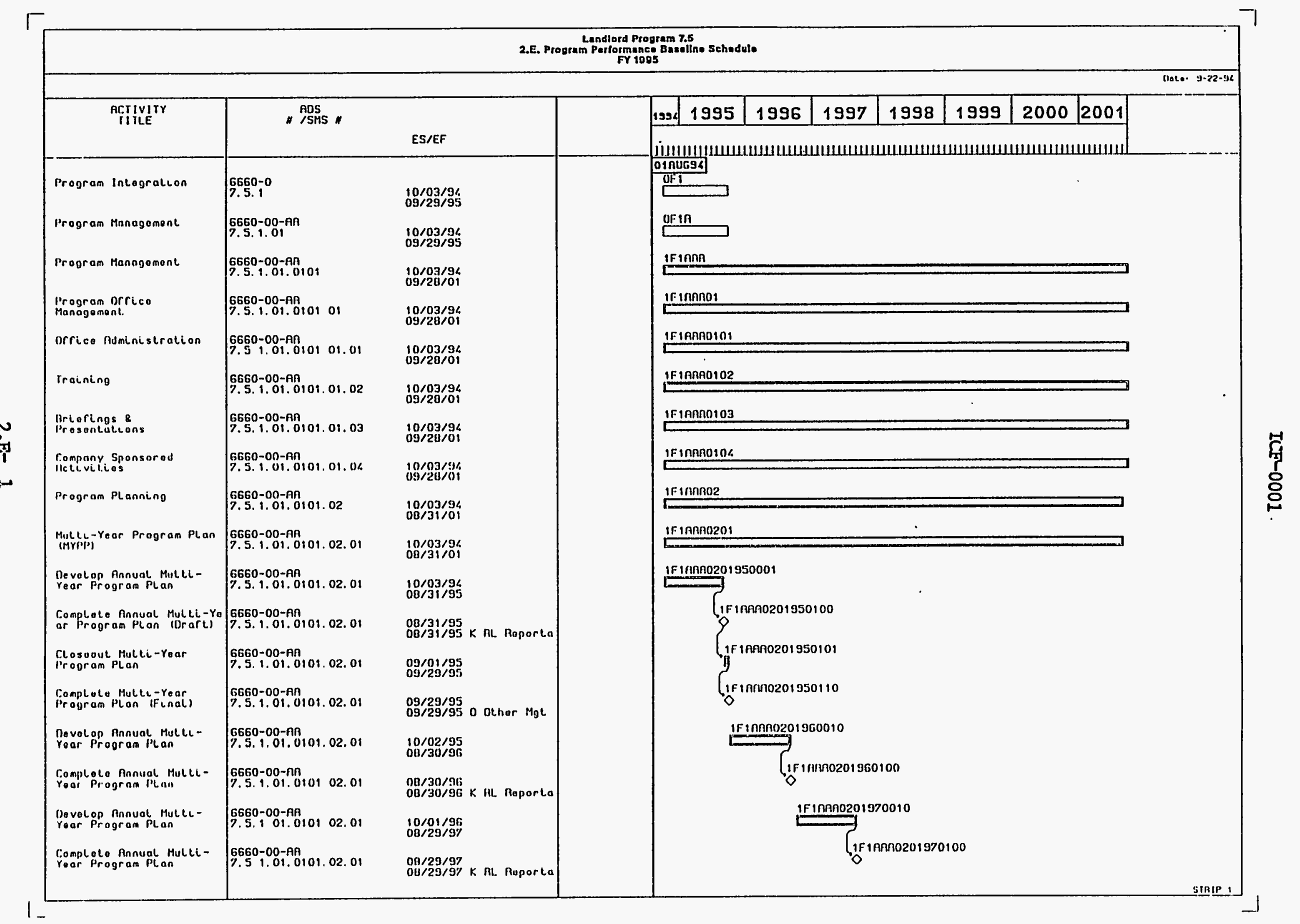


ICF-0001

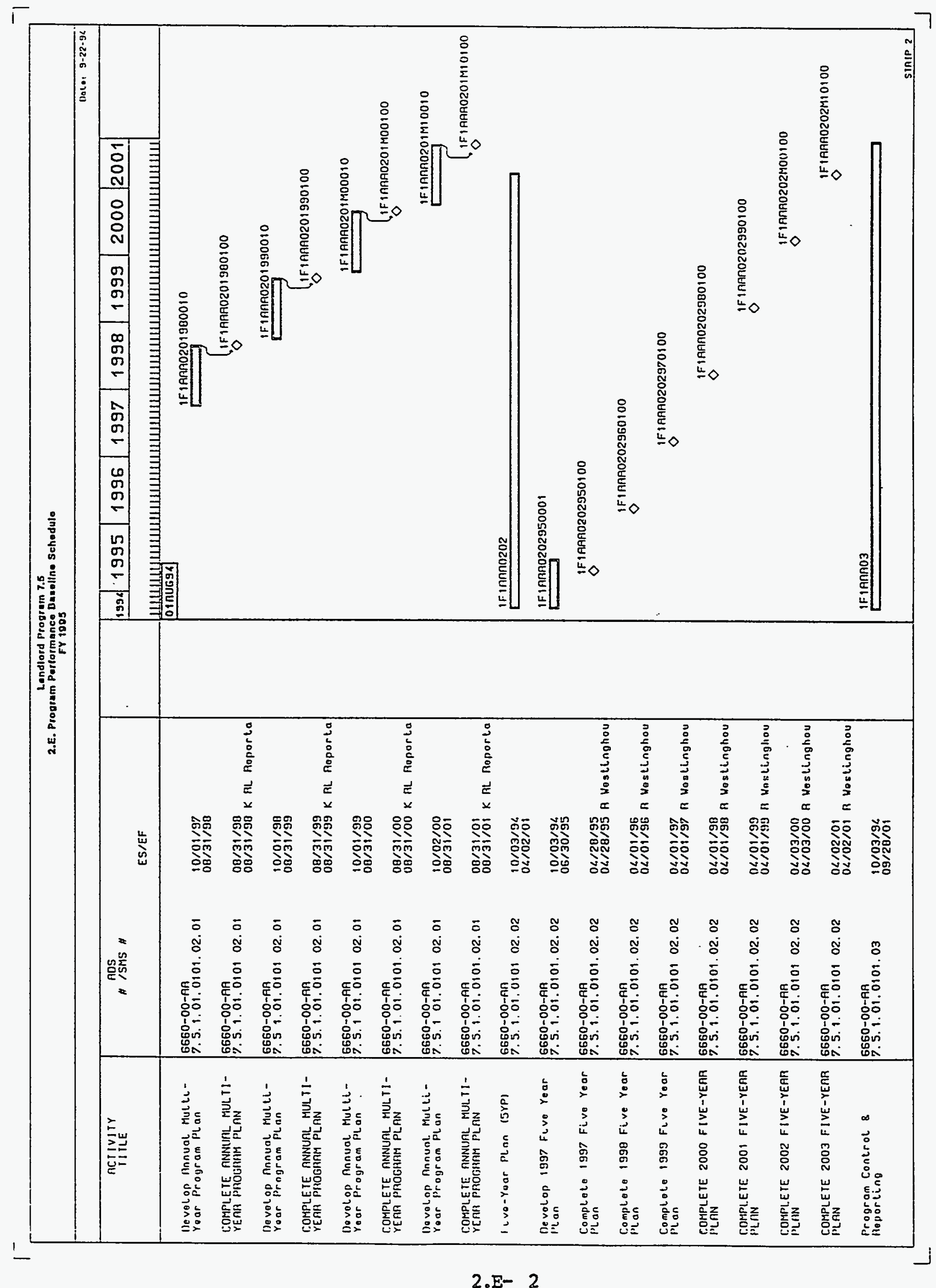




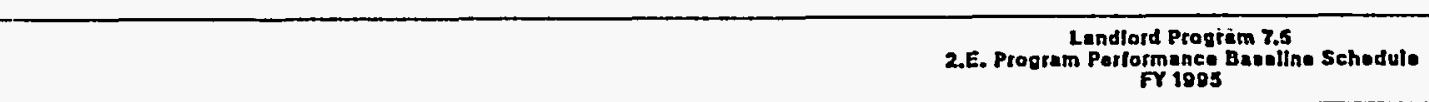

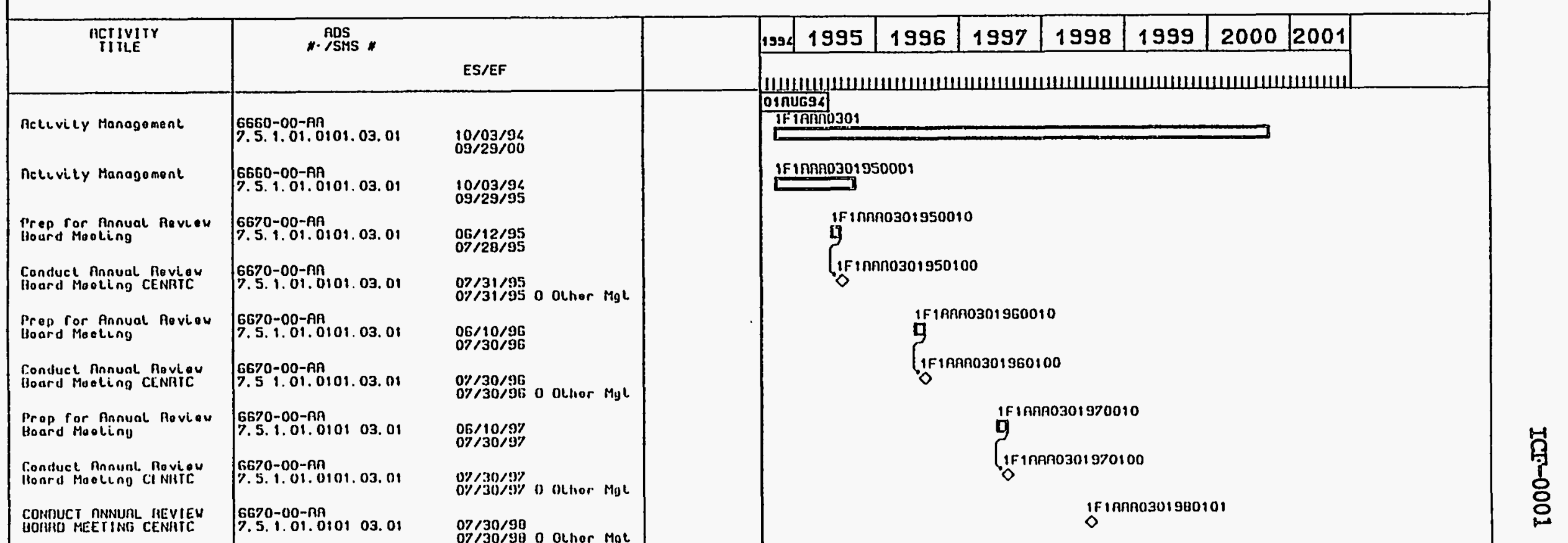

CONDUC. MONIHLY STATUS IINGS CENHIC

6670-00-9A
7.5 .1 .01 .0101 .03 .01

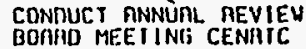
CONDUC, T MONIMLY STATUS MELIINGS CENRIC

6670-00-A9
$7.5,1.01 .0101 .03 .01$ 30/98 o Olhor Mge $09 / 30 / 9 B$
$09 / 30 / 9 B$ $0 \% / 30 / 99$ o olhor Mgl 6670-00-An
7.5 .1 .01 .0101 .03 .01 CONDUC T ANNURL REVIEN

CONDUCT MONIHLY STATUS MEL IINGS EENRTC

6670-00-คก

6670-00-คn

6670-00-An
7.5 .1 .01 .0101 .09 .01 SHS/PIS

$6660-00-A 9$
7.5 .1 .01 .0101 .03 .02

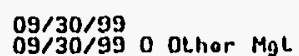

$07 / 28 / 00$
$0 \% / 28 / 00$ o ol.hor Mgt. $09 / 29 / 00$
$09 / 29 / 00$ SHS/PIS $6660-00-$ ค月
7.5 1.01.0101.03.02 $10 / 09 / 94$
$09 / 28 / 01$

10/03/94 Complote Fy 1995 Monthly SMS Roporls 6660-00-คค
7.5 .1 .01 .0101 .03 .02 Complot.e FY
PIS Reports 995 Montialy 6660-00-

7. 5. 1.01.0101.03. 02

03/29/95 o othor Mgt

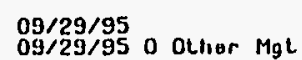




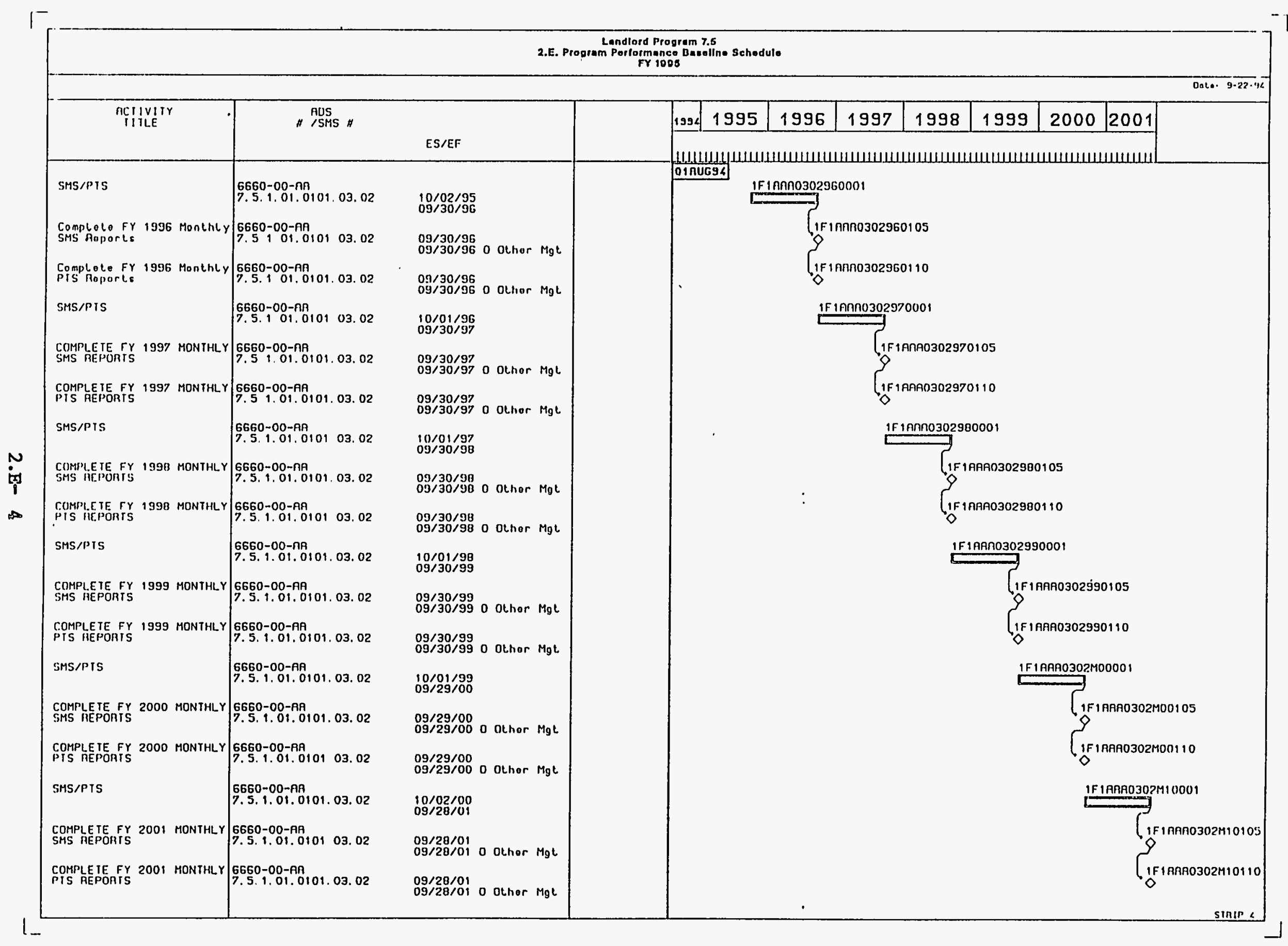




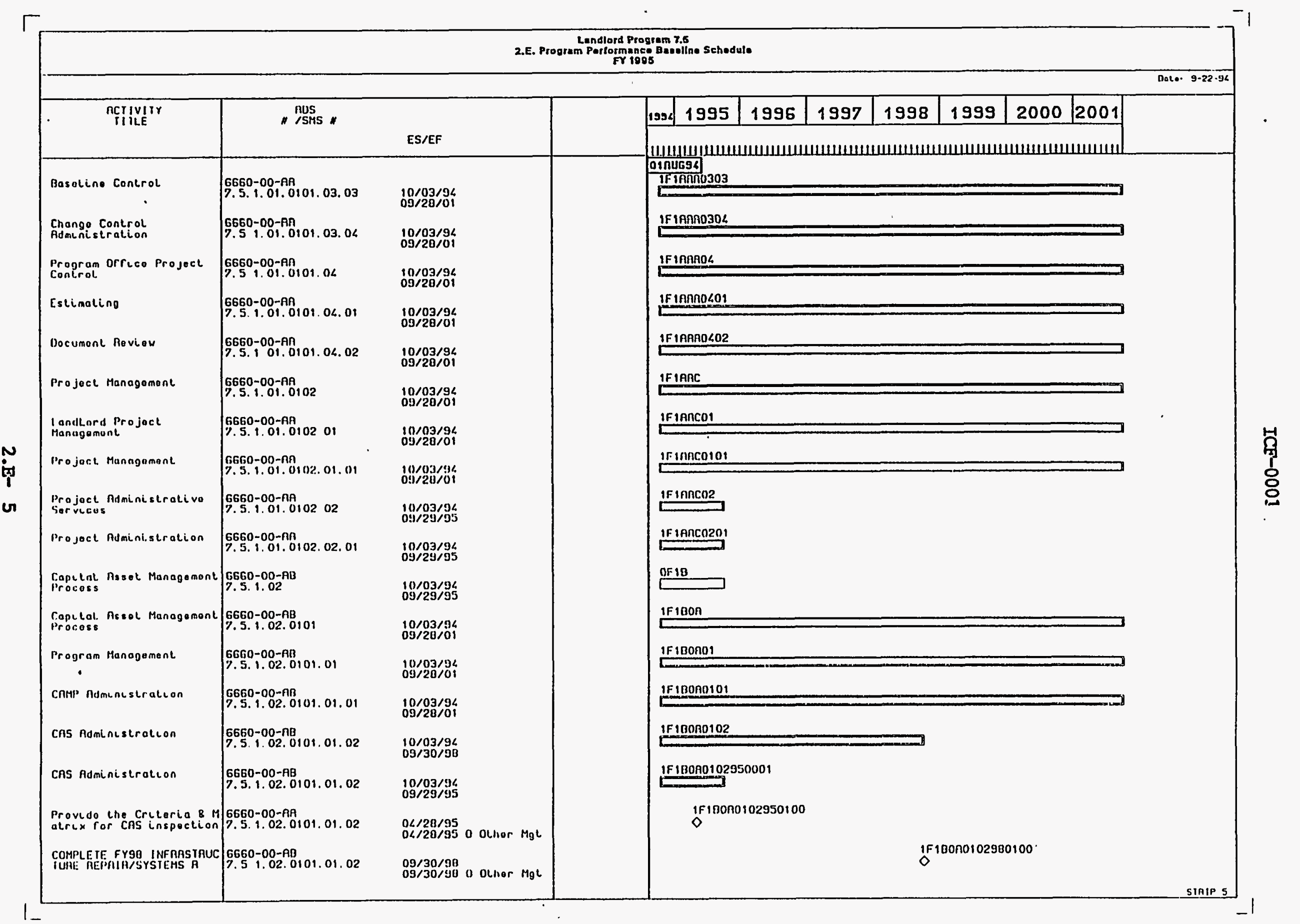




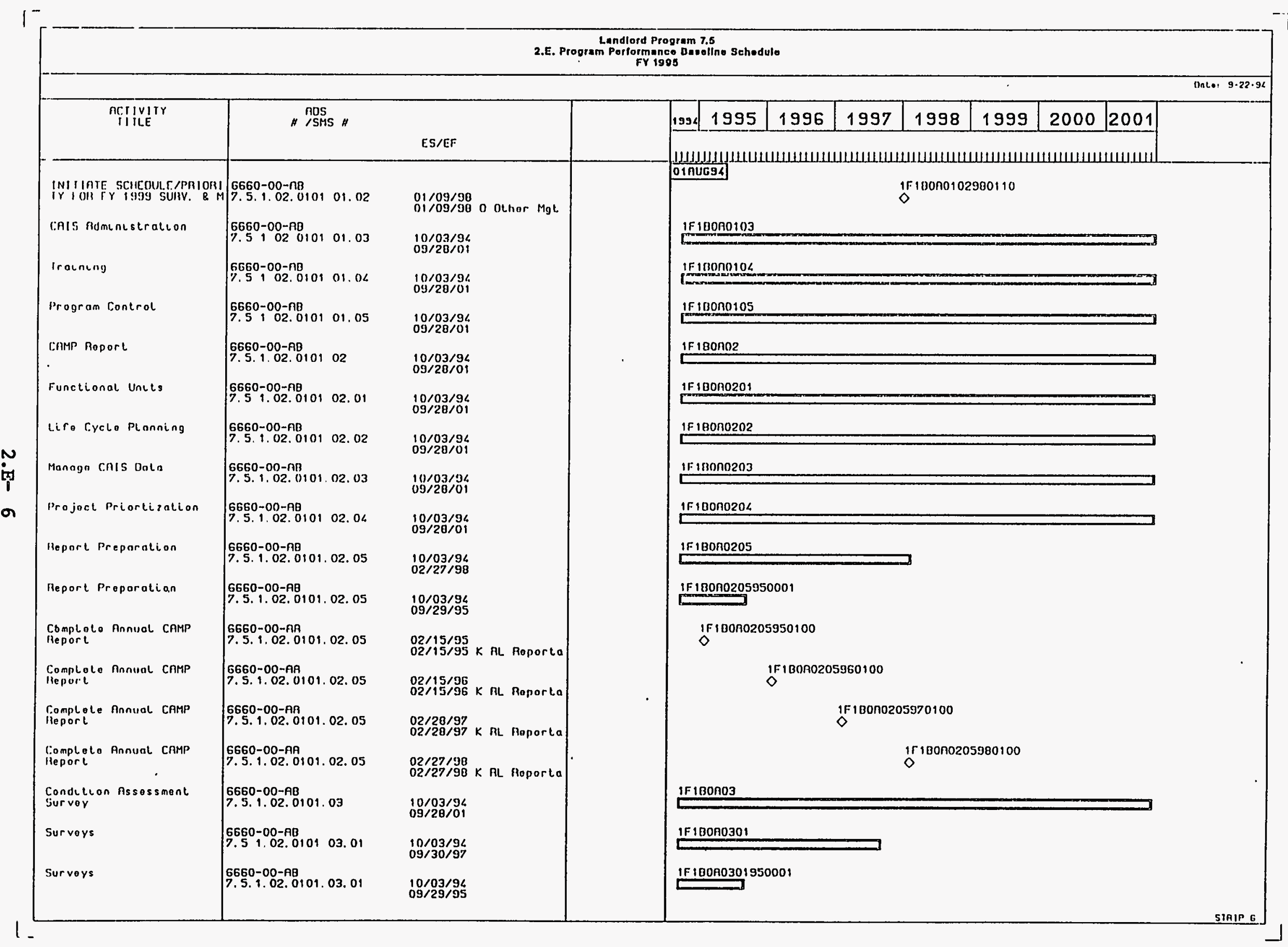




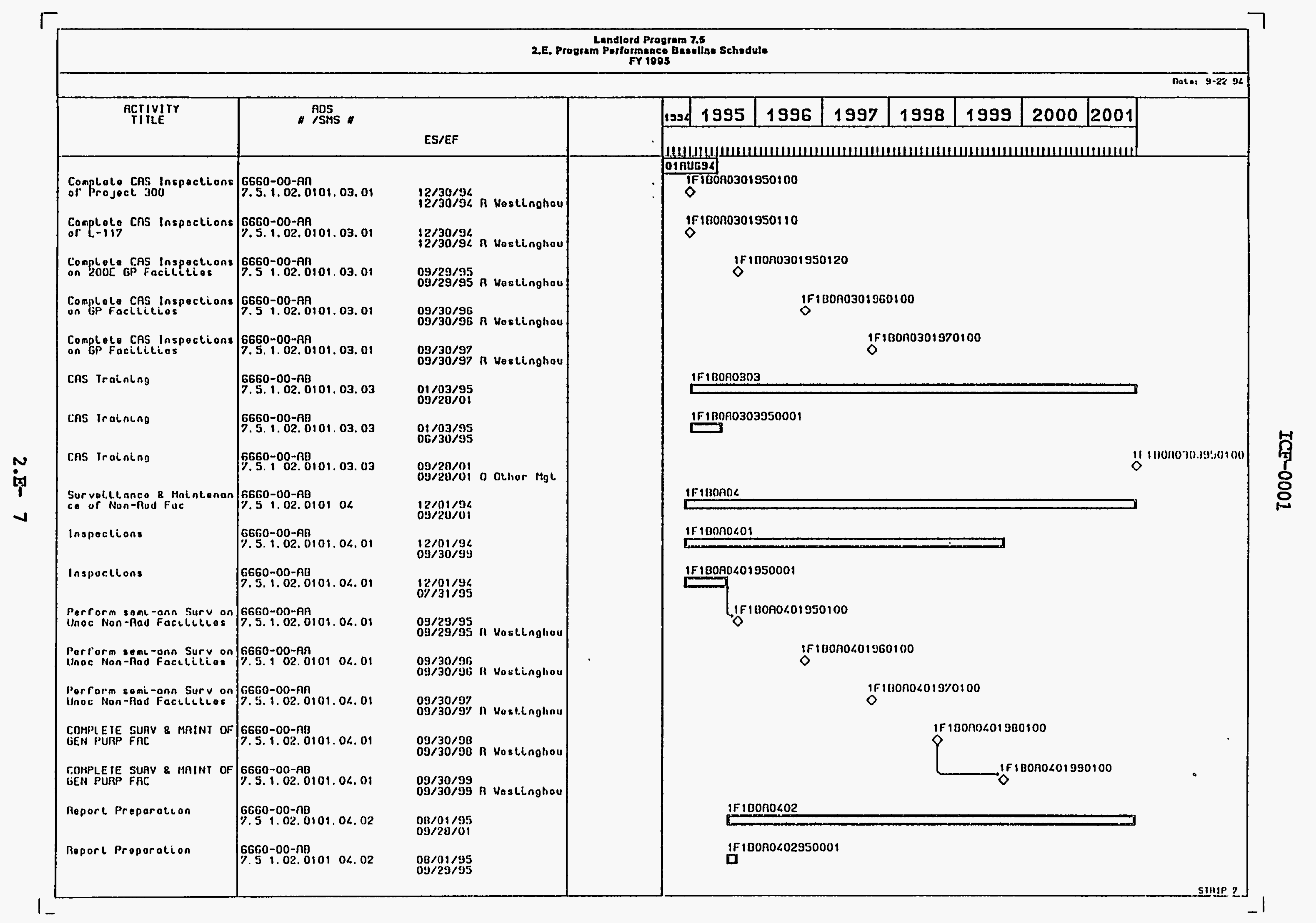




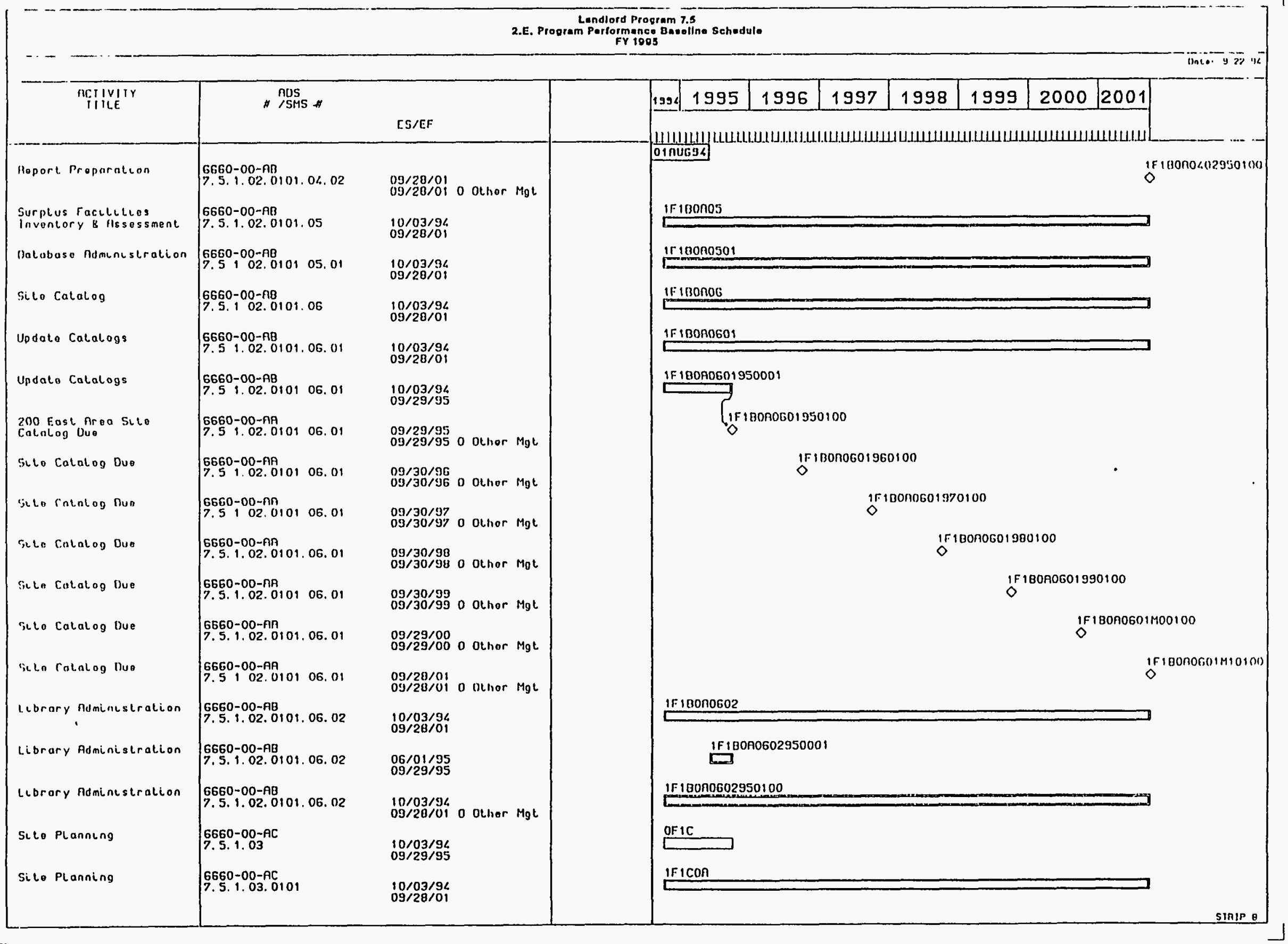


ICF-0001

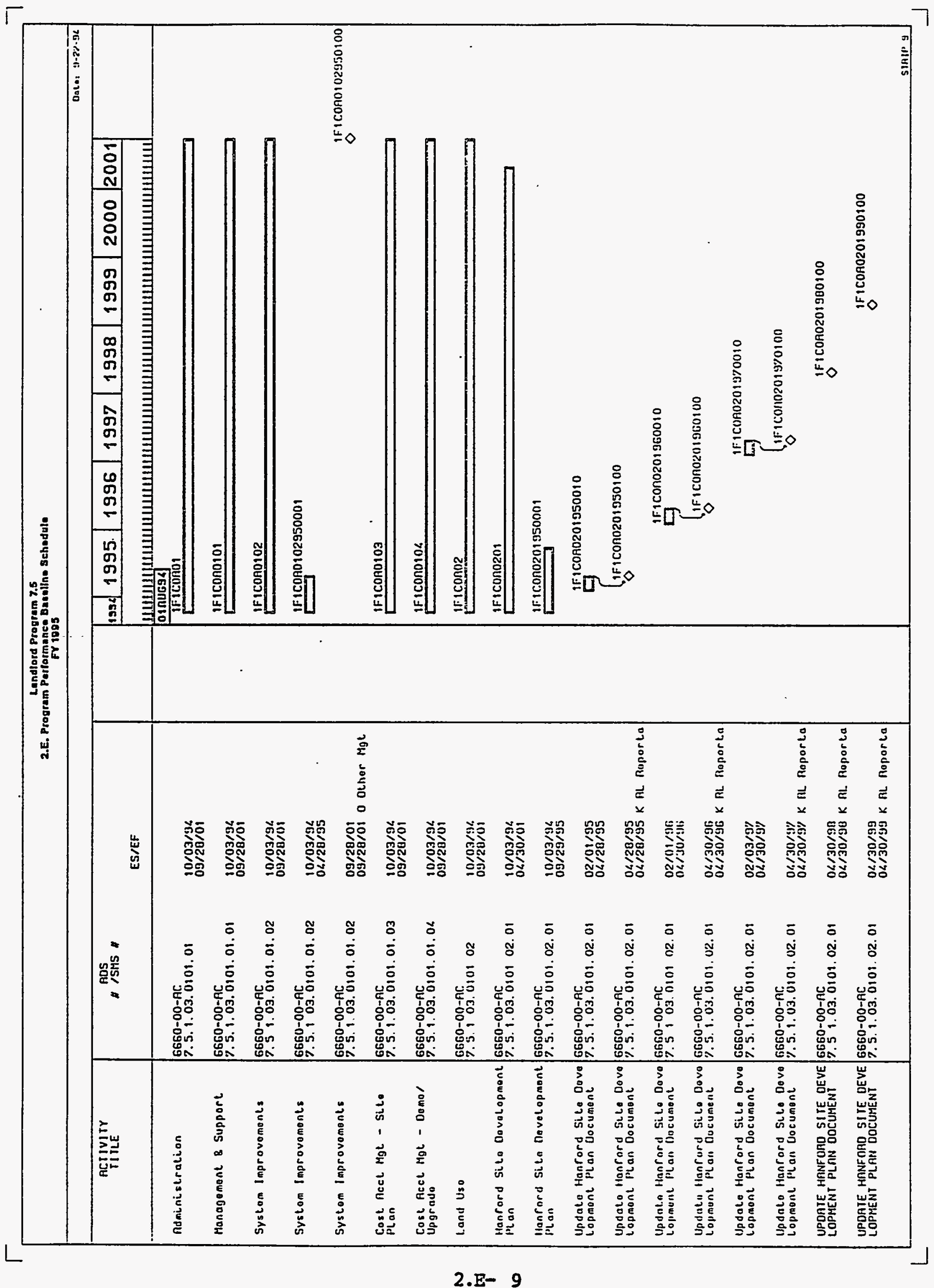




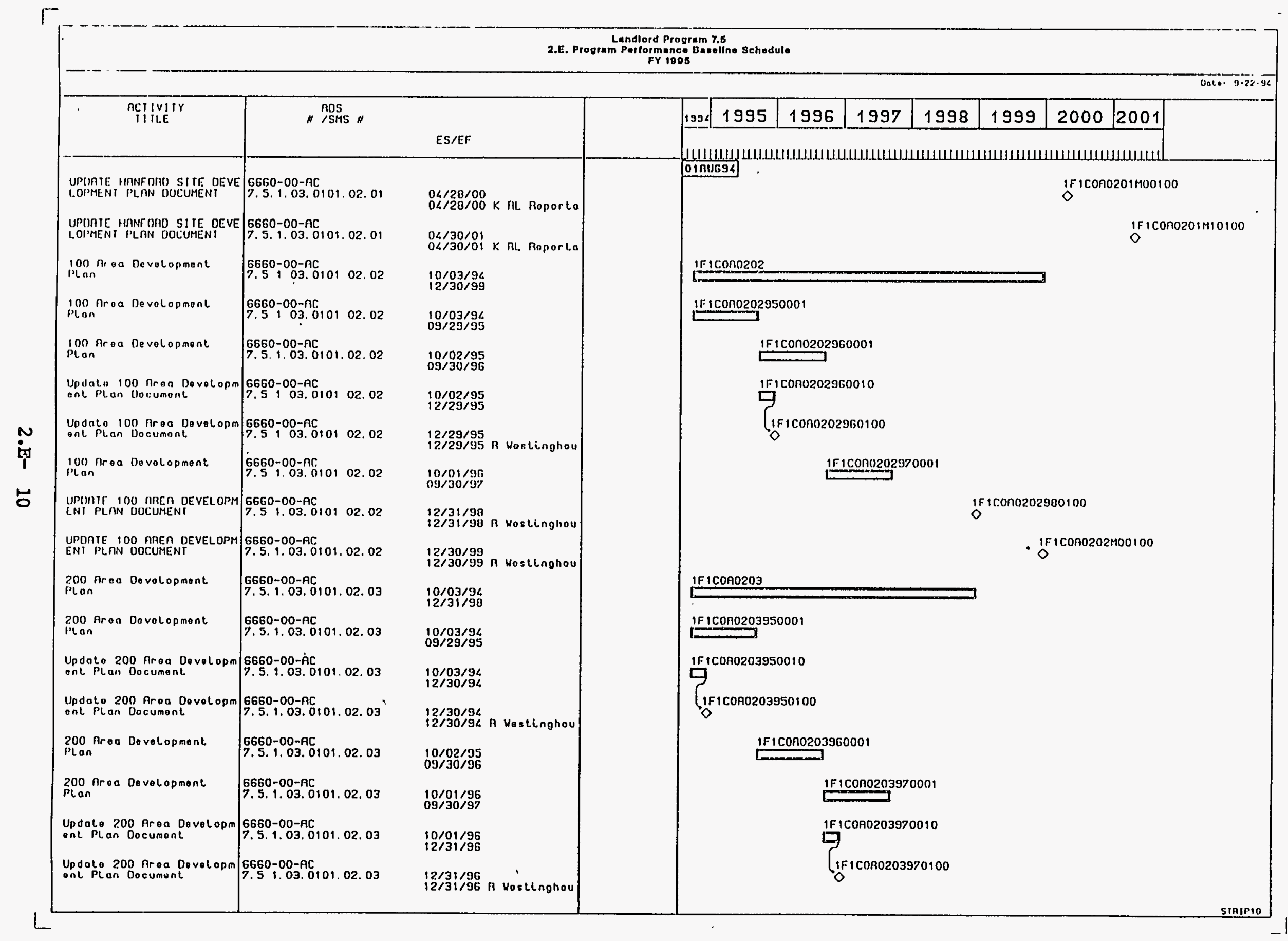




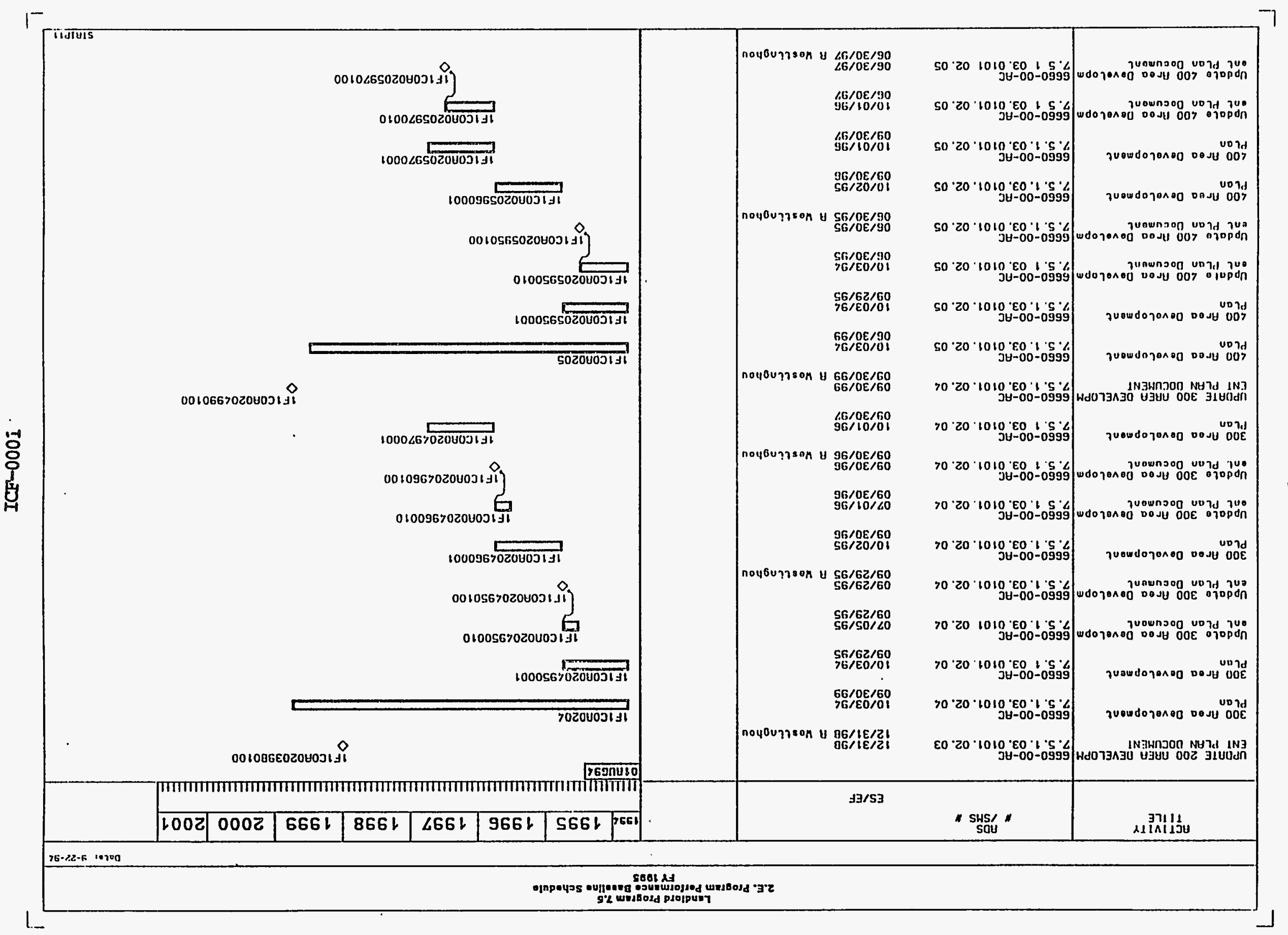




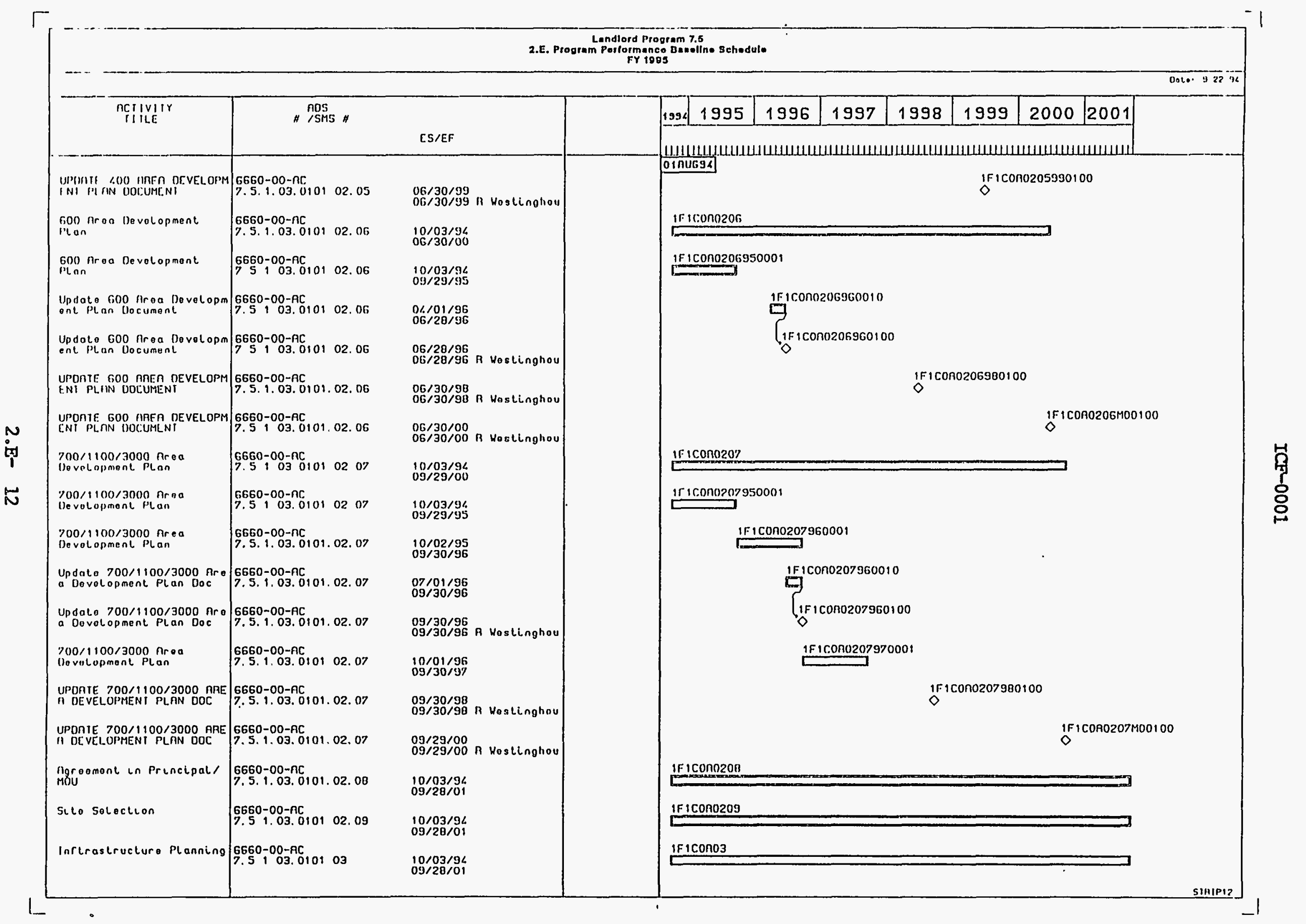




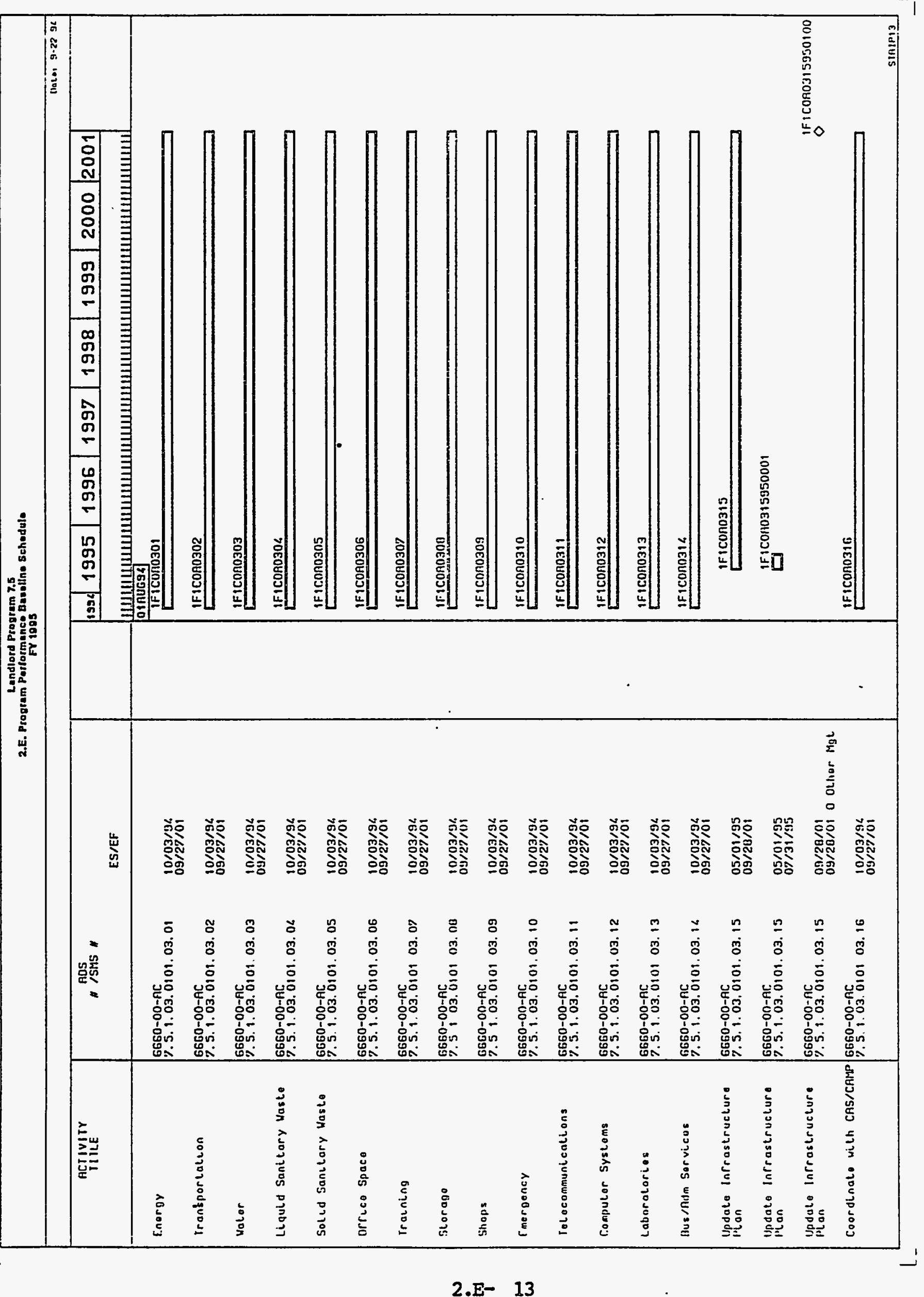




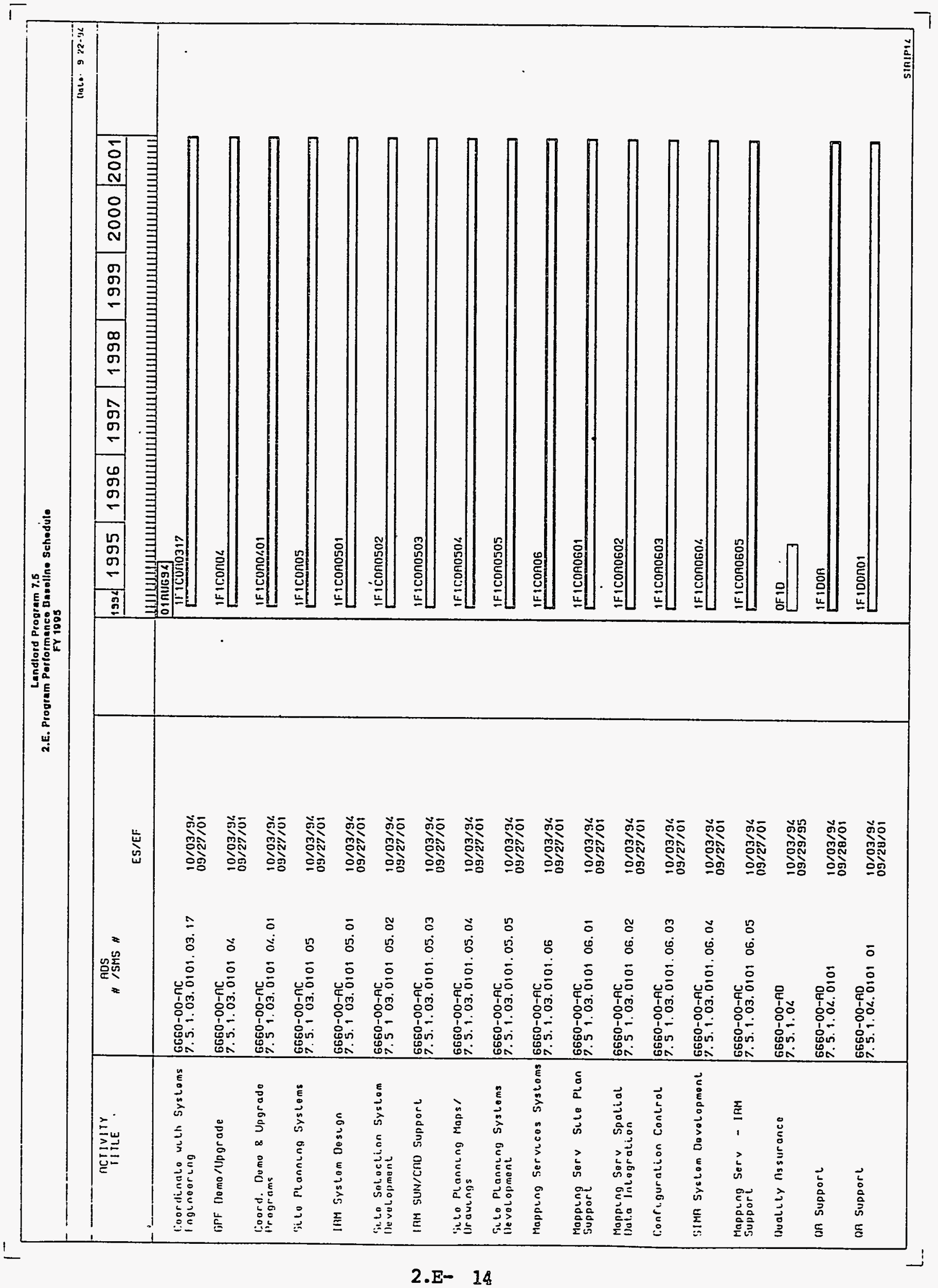




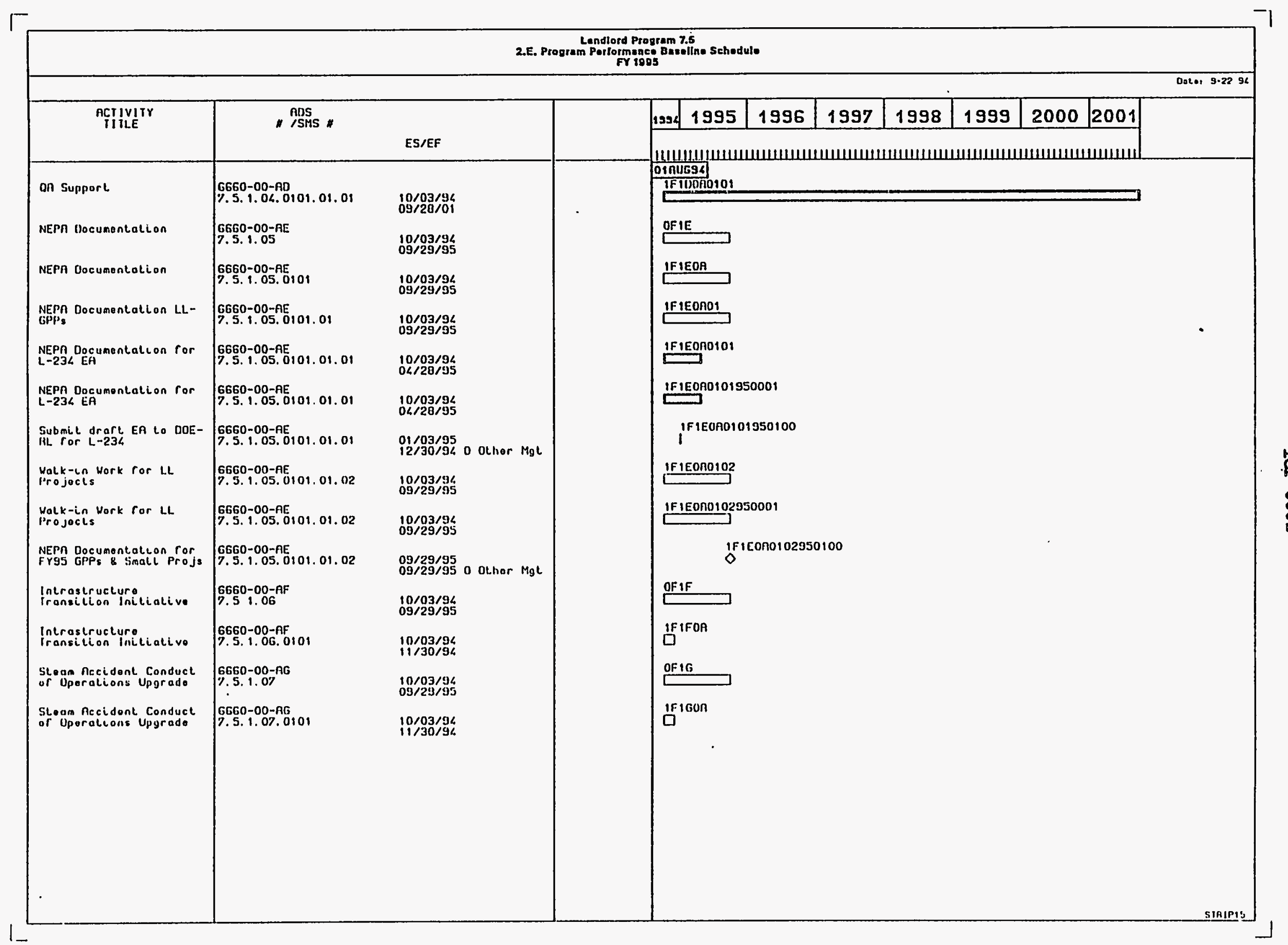




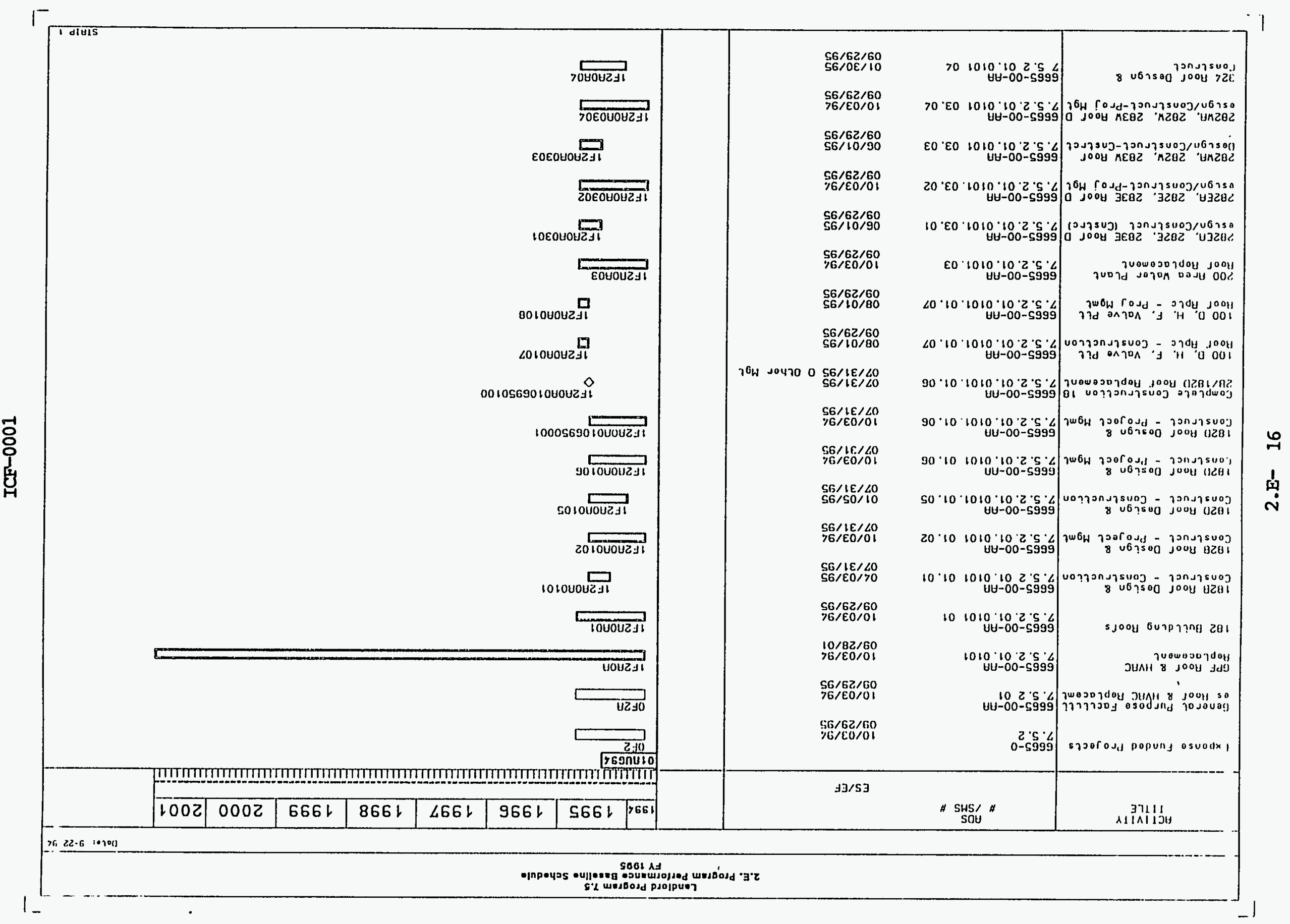




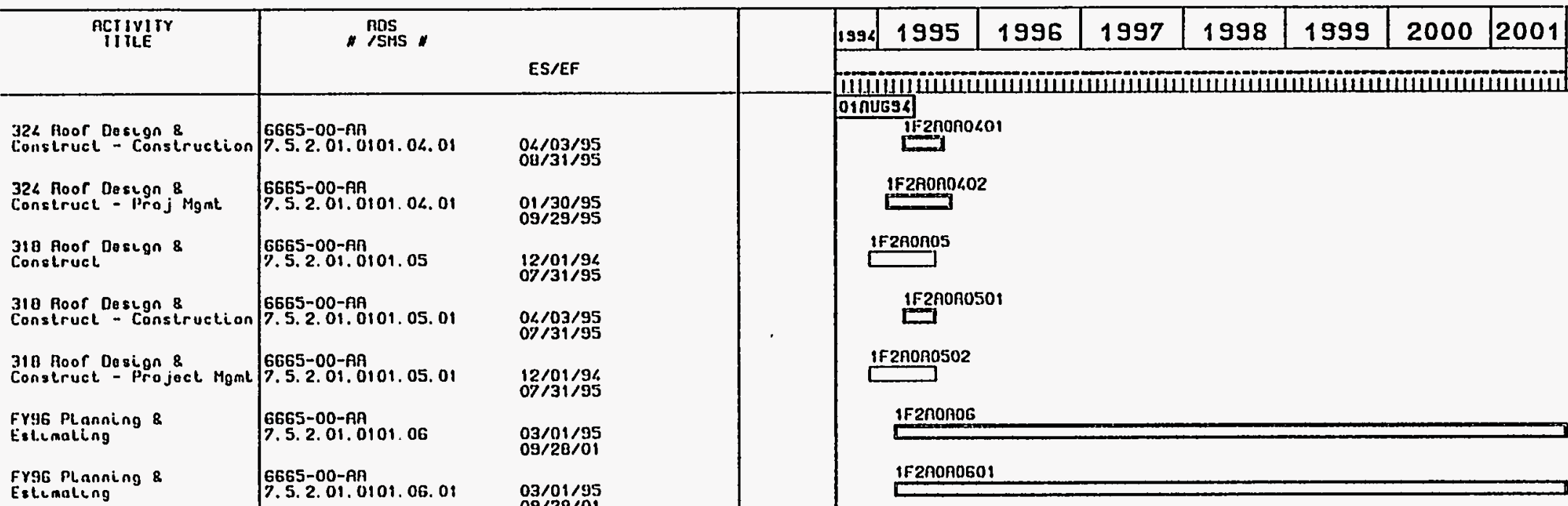

$\begin{array}{ll}\text { Comp FYgG Projocts PLnin } & 6665-00-A \text { A } \\ 9 \text { \& sub schoriostimatos } & \text { 7.5.2.01.0101,06.01 }\end{array}$

$06 / 30 / 95$
$06 / 30 / 95$ o outior Mol

Comp Frg7 Projocts PL nen
$9665-00-A n$
8 sub schod\%ostimalos
7.5 .2 .01 .0101 .06 .01

Complute Roor \& hyac Pro ges5-00-0

Complate Roor R HVAC Pra
js por planneng for FY965-00-AR
7.5 .2 .01 .0101 .06 .01

Complete noof 8 HVAC Pro 6665-00-An

is por planneng ror Frg7
7.5.2.01.0101, 06. 01

Compluto noor 2 IVAC Pro

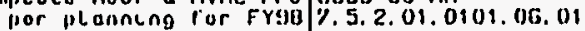

$06 / 28 / 96$ o olher $\mathrm{Mgl}$

$09 / 30 / 965$ o olhar Mgt

$09 / 30 / 97$
$09 / 30 / 97$ o olher Mol

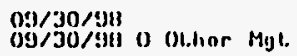

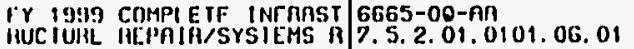

FY 2000 COMPLE TE INFARST
RUCIURE REPRIR/SYSTEMS
6.565-00-AR
7.5 .01 .0101 .06 .01

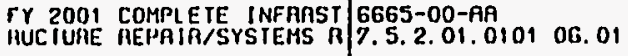

$\begin{array}{lll}\text { General Purpose FacllLLL } & 6665-00-A B \\ \text { os Maintenance \& Repalrs } & 7.5 .2 .02\end{array}$

GPF Ropair 8 Minor

$6665-00-A B$

7.5. 202.0101

09/30/99 o othor Mgt

09/29/00 o ollier MgL

$09 / 23 / 01$

Og/aOrOH O OLhar MgL

$10103 / 94$

Upgrados

$10 / 03 / 96$
$09 / 29 / 95$

\section{F2A0R0601950001 \\ प \\ IF2ROAOGO1950110}

\section{IF2AOROG01960100 \\ If2ROR0601960190}

1r2nonog01970190

1F2n0ก0601300190

$\stackrel{\text { TF 2AOกOG019Y01:10 }}{8}$

IF $_{O}$ AOAOGOIMOOISO

If 2nonotsosmerso

OF21

IF2BOO 


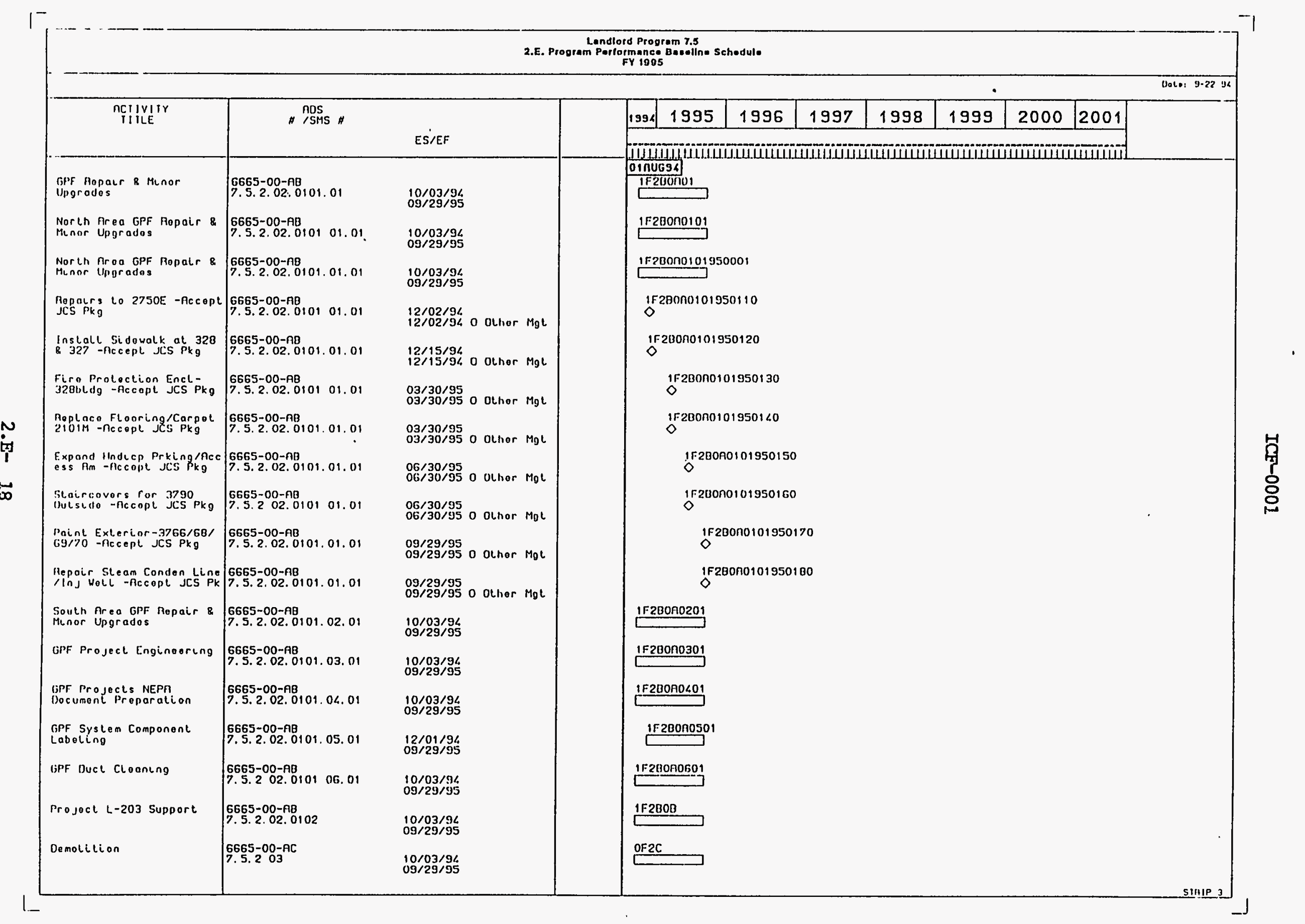




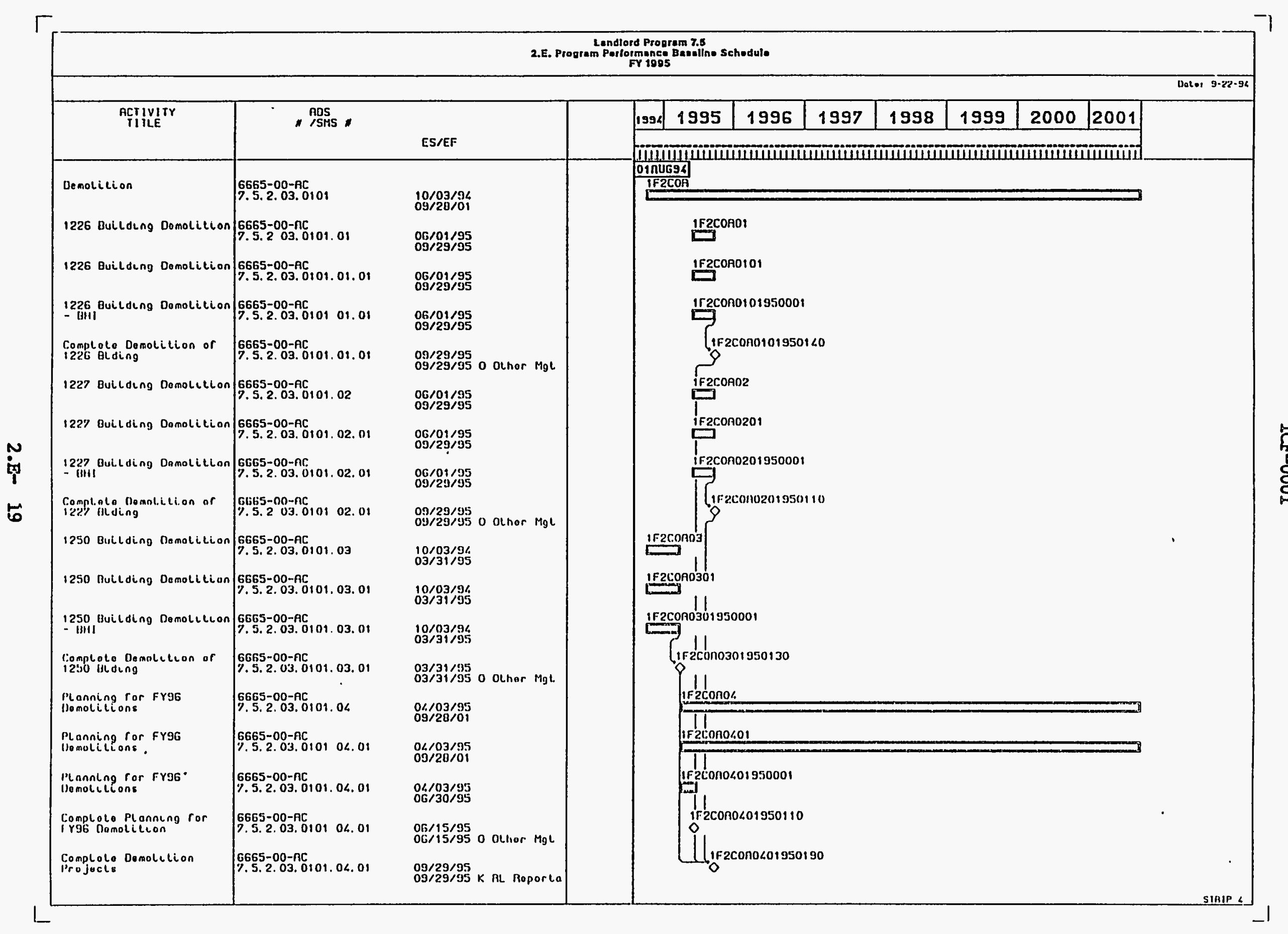




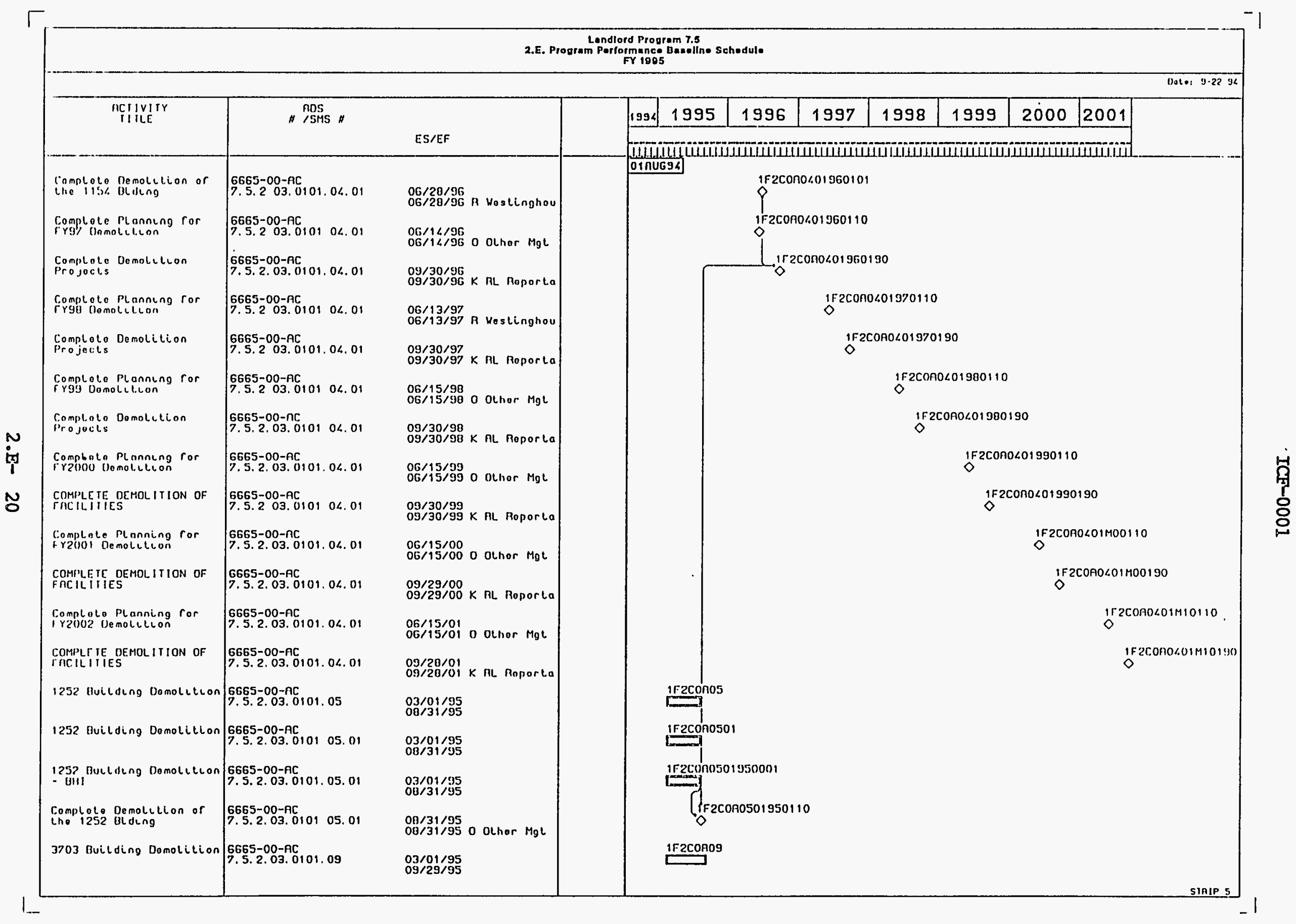


2.E. Program Porformance FY 1905

Dutor 9-22-96

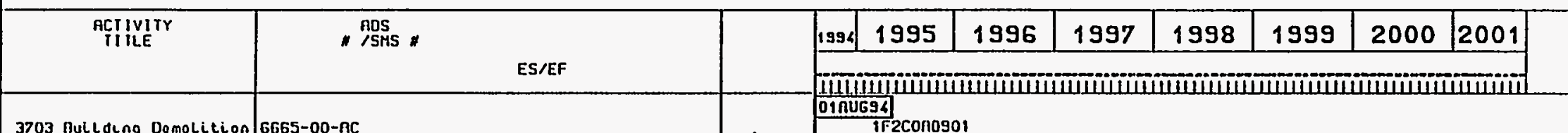

3703 Bulldeng Domolition $\begin{aligned} & \text { 6565-00-AC } \\ & 7.5 .2 .03 .0101 .09 .01\end{aligned}$

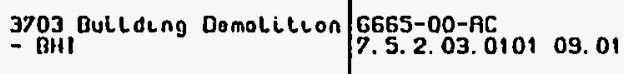

Complete Demolition or
Line j\%03 oldung

271 gun Buil dung
demolittion

271940 Building
Oemolition

27igua Bucloseng
Domolition - yHi

Complute nomolition or

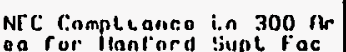

NEC

$03 / 01 / 95$
$09 / 29 / 95$

03/01/95

G665-00-AC
7.5.2.03.0101.09.01

6665-00-AC 10

6665-00-AC
7.5.2.03.0101.10.01

6665-00-AC
$7.5 .2 .03 .0101,10.01$

6665-00-AC

7.5.2.03.0101 10.01

(5)G55-00-80)

. 2.04

6665 -00-An
7.5. 2.04.0101

6665-00-A0
7.5.2.04.0101.01

NEC Compliance

NEC Compllance 300 Aron

NEC Camplianco 300 Aroa

$6665-00-A D$
5. 2.04.0101.01. 01

6665-00-AD

7.5. 2.04.0101.01.01

Compluto NEC Coda Corroc

Lion Nork in tho 324 7.5. 2.04.0101.01.01

Comp 63X or NEC Code Cor 6665-00-AD

eclion work in 325 Btdo 7.5.2.04.0101.01.01

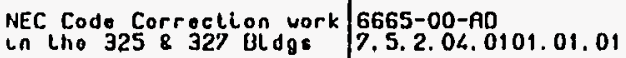

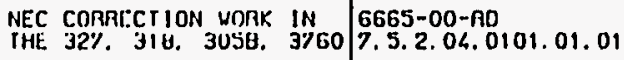

COMPLE IE NEC CORRECTION

$6665-00-70$
7.5 .204 .0101 .01 .01

Saroly Rnalysle Roporls

$6665-00-A E$
7.5 .2 .05
$09 / 29 / 95$
$09 / 23 / 95$

$03 / 01 / 95$
$07 / 31 / 95$

$03 / 01 / 95$
$07 / 31 / 95$

$03 / 01 / 95$
$07 / 31 / 95$

$07 / 31 / 95$ o olhor MgL

$10 / 0.3 / 9)$
$09 / 29 / 513$

$10 / 03 / 94$
$05 / 30 / 99$

$10 / 03 / 94$
$03 / 30 / 99$

$10 / 03 / 94$
$09 / 30 / 99$

$10 / 03 / 94$
$09 / 29 / 95$

$09 / 29 / 95$
$09 / 29 / 95$ o othor Mgt

$09 / 30 / 96$

09/30/97 n wostlinghou

$09 / 30 / 98$

/30/90 in Wogllnghou

$09 / 30 / 99$

is Heslingliou

$10 / 03 / 94$
$09 / 29 / 95$
01RUG94

IF2COnOSO

1F2COR0901950001

(

IF2COROSO1950110

1F2Con10

IF2COA100

1F2CON100195000

$\longrightarrow$

yraconto019501 to

OF?

IF\%UOA

1 F200no1

IF200ADIOI

IF200R0101950001

IF2DOR0101350100

$\stackrel{1 F 20000101960110}{8}$

1F200no101970110

$15200 n 0101980110$

$\stackrel{1 F 200 A 0101990110}{8}$

OF2E 


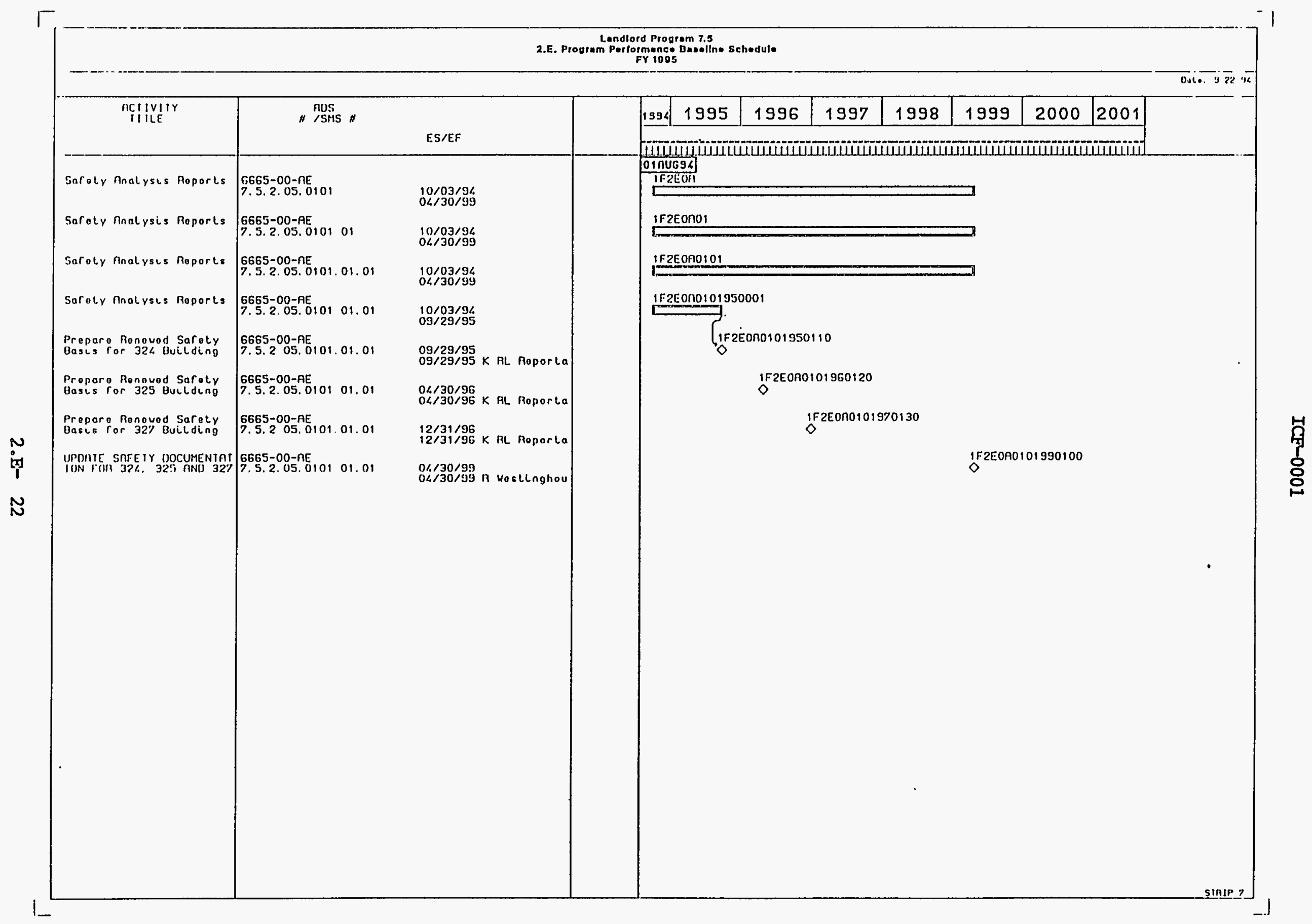




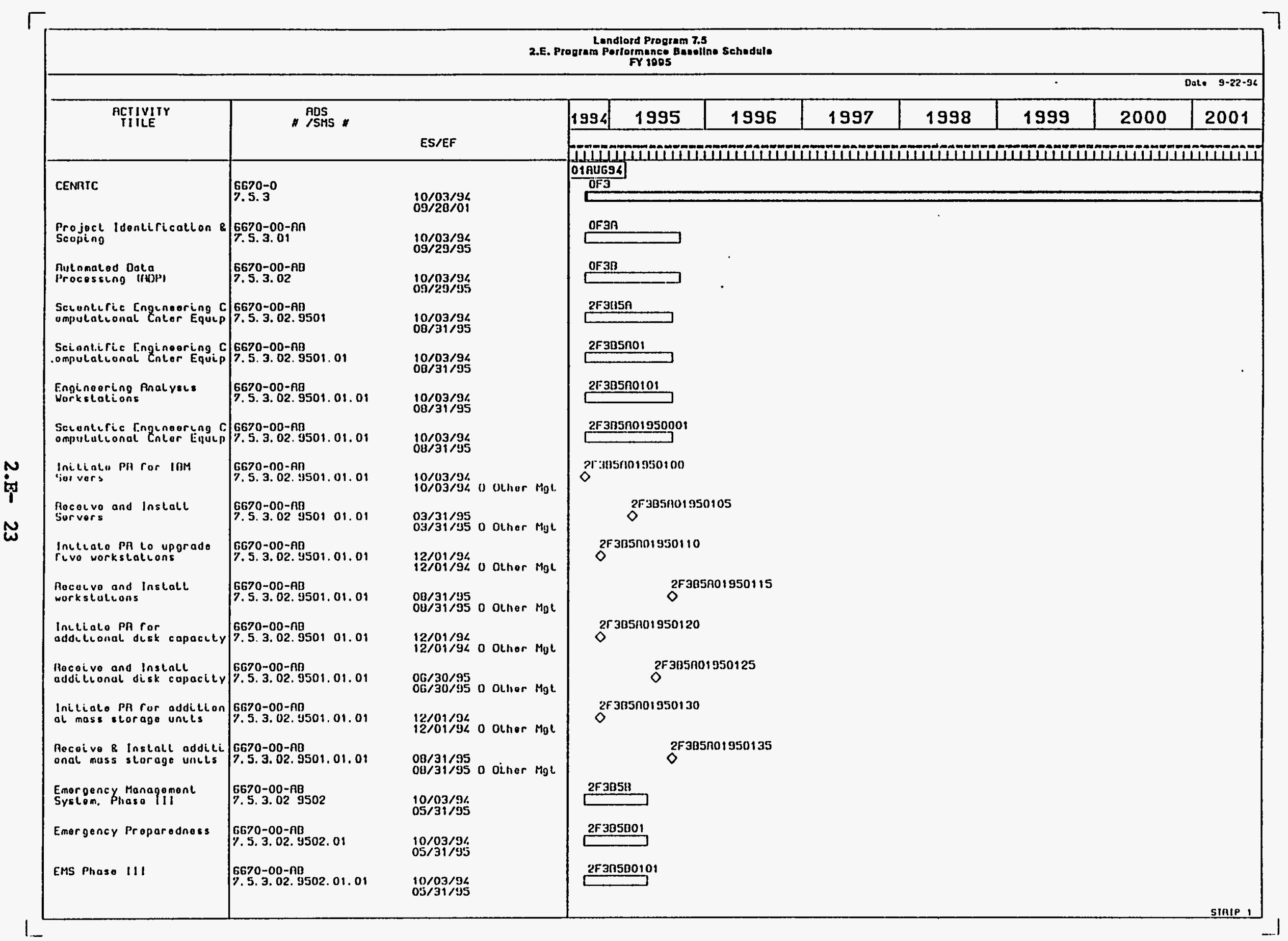




\begin{tabular}{|c|c|c|c|c|c|c|c|c|c|}
\hline \multicolumn{10}{|c|}{ 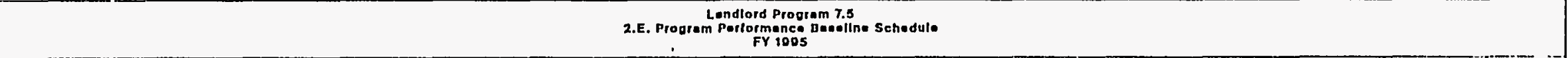 } \\
\hline \multicolumn{10}{|c|}{ Docio :1223 } \\
\hline \multirow[t]{2}{*}{ neilvitr } & \multirow[t]{2}{*}{ * ADS } & \multirow[b]{2}{*}{ ES/EF } & $1994 \quad 1995$ & 1996 & 1997 & 1998 & 1999 & 2000 & 2001 \\
\hline & & & \multicolumn{7}{|c|}{ 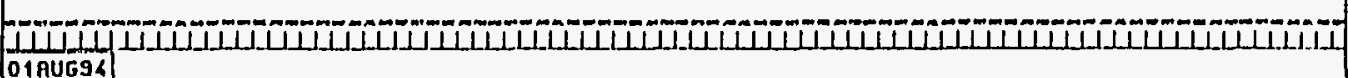 } \\
\hline TME Phaso III & $\begin{array}{l}6670-00-n \theta \\
7.5 .3 .02 .9502 .01 .01\end{array}$ & $\begin{array}{l}\text { 10/03/94 } \\
05 / 31 / 95\end{array}$ & 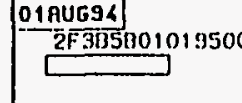 & & & & & & 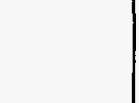 \\
\hline 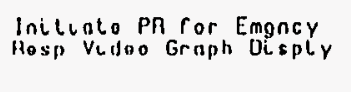 & 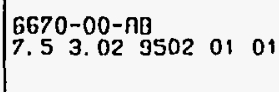 & 11/01/94 o other Mgt & $\stackrel{\diamond}{O}^{2 F 38580101950}$ & & & & & & \\
\hline $\begin{array}{l}\text { Racolvo \& Install Emgney } \\
\text { noso Vidoo Graph Disply }\end{array}$ & $\begin{array}{l}6670-00-n B \\
7.5 .3 .02 .9502 .01 .01\end{array}$ & $\begin{array}{l}03 / 01 / 95 \\
03 / 01 / 95 \text { o other } \mathrm{Mgl}\end{array}$ & $\delta_{0}^{2 F 3858010}$ & & & & & & \\
\hline $\begin{array}{l}\text { InLliale PR for Monctors } \\
\text { Wrkstations \& Sortuaro }\end{array}$ & $\begin{array}{l}6670-00-\text { - } 98 \\
7.5 .3 .02 .9502 .01 .01\end{array}$ & $\begin{array}{l}12 / 01 / 94 \\
12 / 01 / 94 \text { o other MgL }\end{array}$ & $\diamond^{2 F 3853010195}$ & & & & & & \\
\hline $\begin{array}{l}\text { Rocolve \& instanll Monito } \\
\text { rs. Wrkstalions \& Sriuro }\end{array}$ & 7.53 .029502 .01 .01 & $05 / 01 / 95$ o olher MgL & $\delta^{2 F 385 B C}$ & 30115 & & & & & \\
\hline $\begin{array}{l}\text { Goographic } \ln _{\text {Grormation }} \\
\text { Sysiom, Phaso }\end{array}$ & $\begin{array}{l}\text { 6670-00-คB } \\
7.5 .3 .02 .9503\end{array}$ & $\begin{array}{l}10 / 03 / 96 \\
05 / 31 / 95\end{array}$ & $\stackrel{2 F 3 B 5 C}{\longleftarrow}$ & & & & & & \\
\hline $\begin{array}{l}\text { Spatial Dato lnfo Mgl } \\
\text { System }\end{array}$ & $\begin{array}{l}6670-00-\text {-A8 } \\
7.5 .3 .02 .9503 .01\end{array}$ & $\begin{array}{l}\text { 10/03/9/6 } \\
05 / 31 / 95\end{array}$ & & & & & & & \\
\hline $\begin{array}{l}\text { Spalial Dal., informalion } \\
\text { Mrymt siystom }\end{array}$ & $\begin{array}{l}6670-00-\text { - } 19 \\
7.5 .3 .02 .0503 .01 .01\end{array}$ & $\begin{array}{l}10 / 03 / 94 \\
055 / 31 / 95\end{array}$ & $\stackrel{2539560109}{=}$ & & & & & & \\
\hline $\begin{array}{l}\text { racillelios nLr } \\
\text { condulionors } 121\end{array}$ & 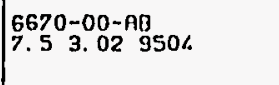 & $\begin{array}{l}12 / 30 / 96 \\
04 / 28 / 95\end{array}$ & $\stackrel{2 F 3450}{\longleftarrow}$ & & & & & & \\
\hline $\begin{array}{l}\text { Data Conter nur } \\
\text { Condliconers (2) }\end{array}$ & $\begin{array}{l}6670-00-n g \\
7.5 .3 .02 .9504 .01\end{array}$ & $\begin{array}{l}11 / 30 / 96 \\
0</ 28 / 95\end{array}$ & & & & & & & \\
\hline $\begin{array}{l}\text { Duta Corter ALr } \\
\text { Condulioners (2) }\end{array}$ & $\begin{array}{l}6670-00-\text { R日 } \\
7.5 .3 .02 .9504 .01 .01\end{array}$ & $\begin{array}{l}11 / 30 / 94 \\
0</ 28 / 95\end{array}$ & & & & & & & \\
\hline Bucldengs & $\begin{array}{l}6670-00-\mathrm{AC} \\
7.5 .3 .03\end{array}$ & $\begin{array}{l}\text { 10/03/96 } \\
09 / 29 / 95\end{array}$ & $\stackrel{\mathrm{OF} 3 \mathrm{C}}{\longleftarrow}$ & & & & & & \\
\hline Mobile orrucos & $\begin{array}{l}6670-00-A C \\
7.5 .3 .03 .9501\end{array}$ & $\begin{array}{l}10 / 03 / 94 \\
09 / 29 / 95\end{array}$ & $\stackrel{2 F 3 C 5 A}{\longleftarrow}$ & & & & & & \\
\hline Procuro Mobllo orrices & $\begin{array}{l}\text { 6670-00-AC } \\
7.5 .3 .03 .9501 .01\end{array}$ & $\begin{array}{l}\text { 10/03/96 } \\
09 / 29 / 95\end{array}$ & & & & & & & \\
\hline Procure Mobile orficos & $\begin{array}{l}6670-00-\mathrm{AC} \\
7.53 .03 \mathrm{9501} \\
7.51 .09\end{array}$ & $\begin{array}{l}\text { 10/03/94 } \\
09 / 29 / 95\end{array}$ & 2F 3C5R0101 & & & & & & \\
\hline Business Equepmene & $\begin{array}{l}\text { 6670-00-90 } \\
7.5 .3 .04\end{array}$ & $\begin{array}{l}\text { 10/03/92 } \\
09 / 29 / 95\end{array}$ & $\stackrel{0 F 30}{\longleftarrow}$ & & & & & & \\
\hline $\begin{array}{l}\text { Ouscuness \& Informallon } \\
\text { Sorvecos Equipment }\end{array}$ & $\begin{array}{l}\text { 6670-00-A0 } \\
7.5 .3 .04 .9501\end{array}$ & $\begin{array}{l}10 / 31 / 94 \\
09 / 29 / 95\end{array}$ & 2 2F305A & & & & & & \\
\hline 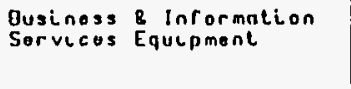 & $\begin{array}{l}6670-00-\mathrm{AD}^{6} \\
\text { 7.5.3.06 } 950101\end{array}$ & $\begin{array}{l}\text { 10/31/96 } \\
09 / 23 / 95\end{array}$ & 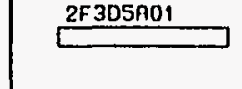 & & & & & & \\
\hline
\end{tabular}




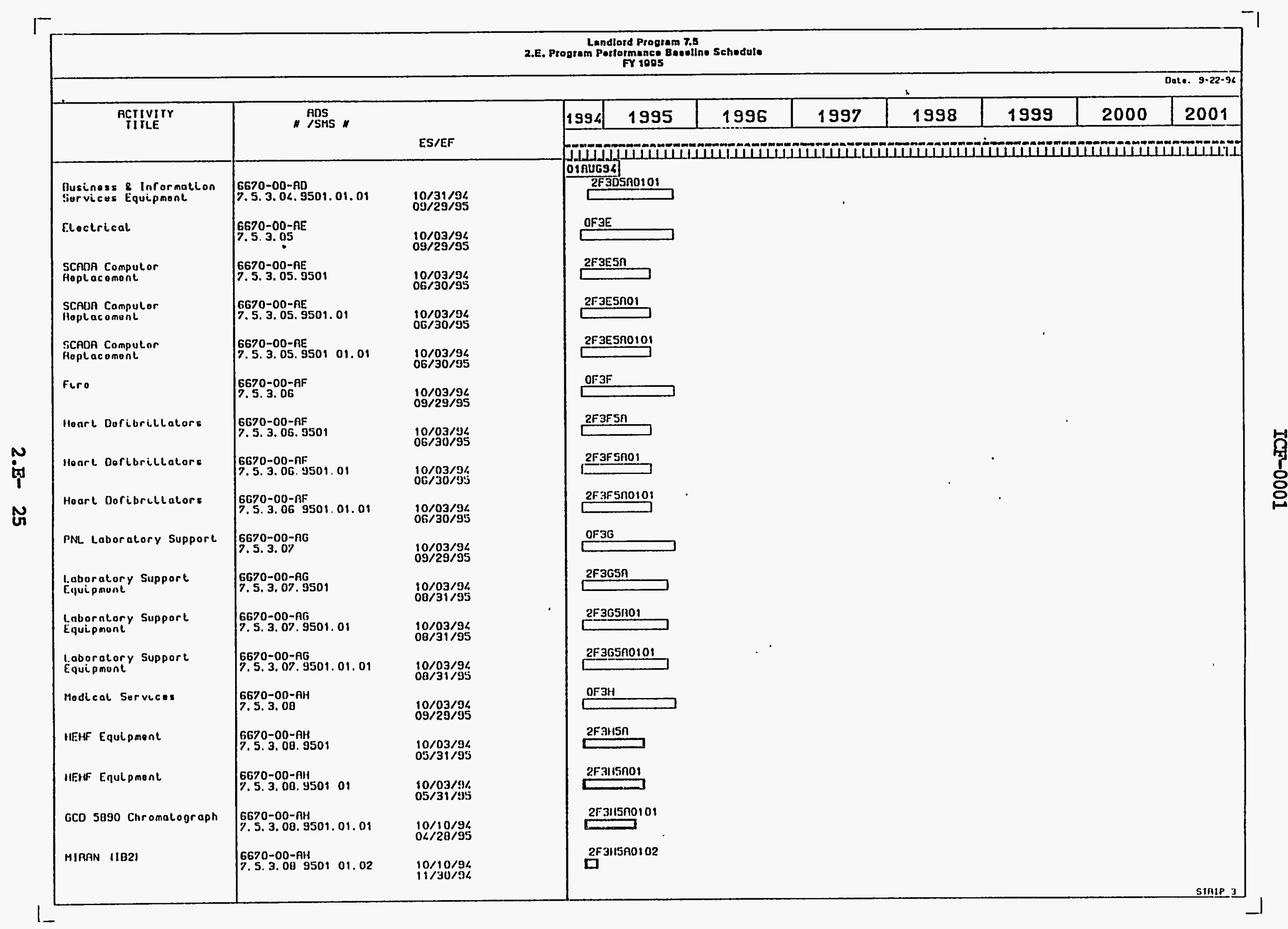




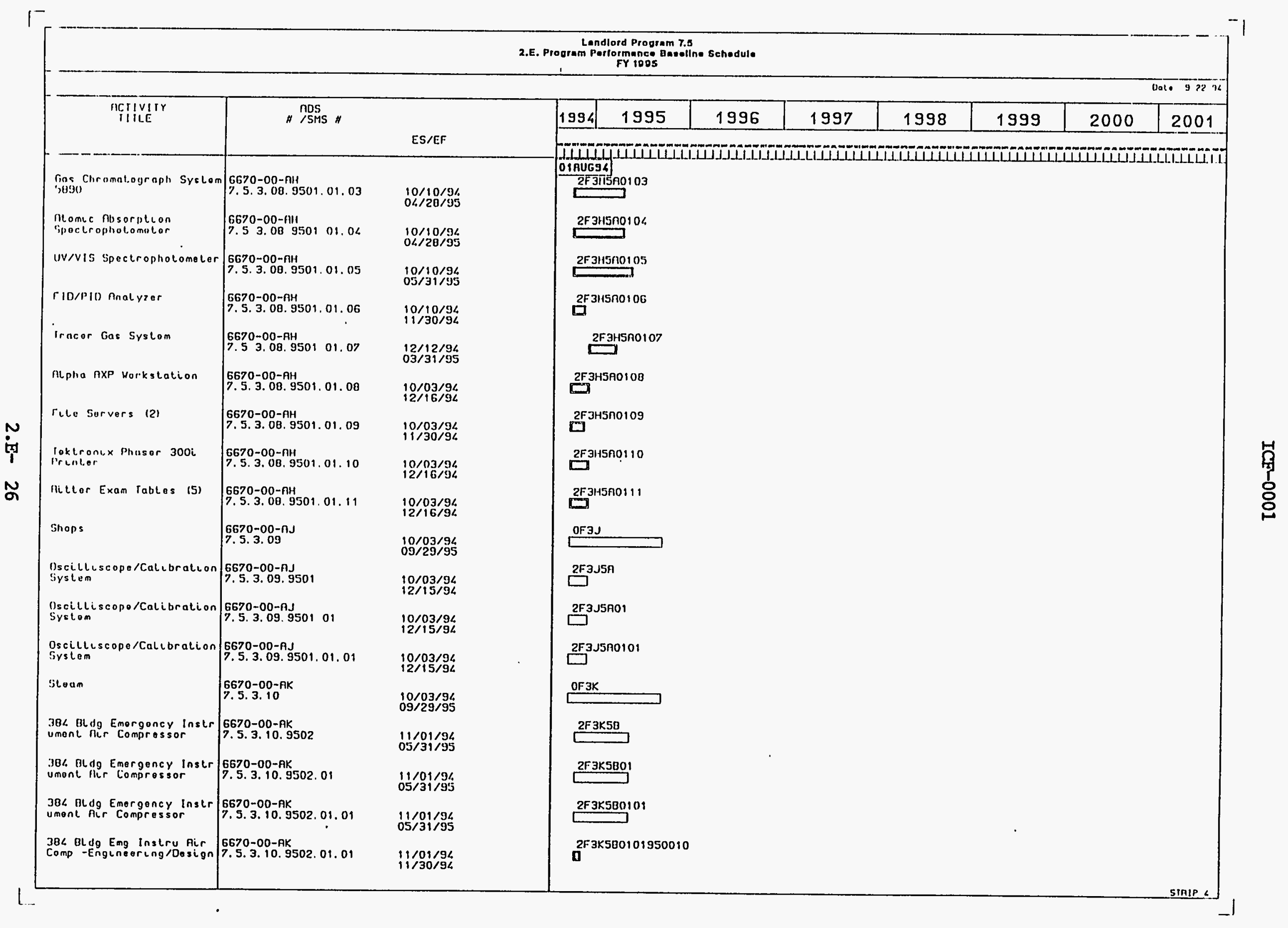




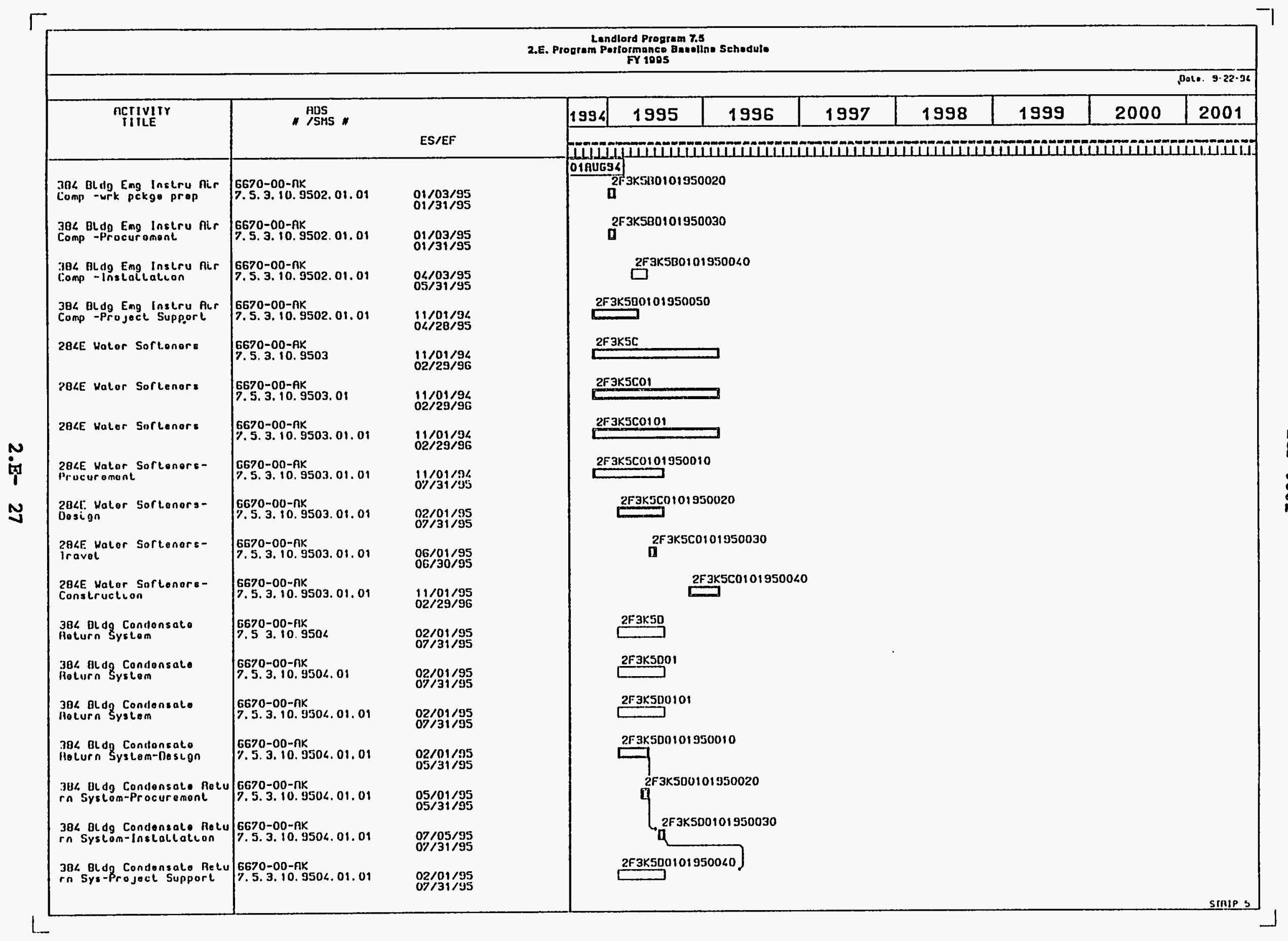




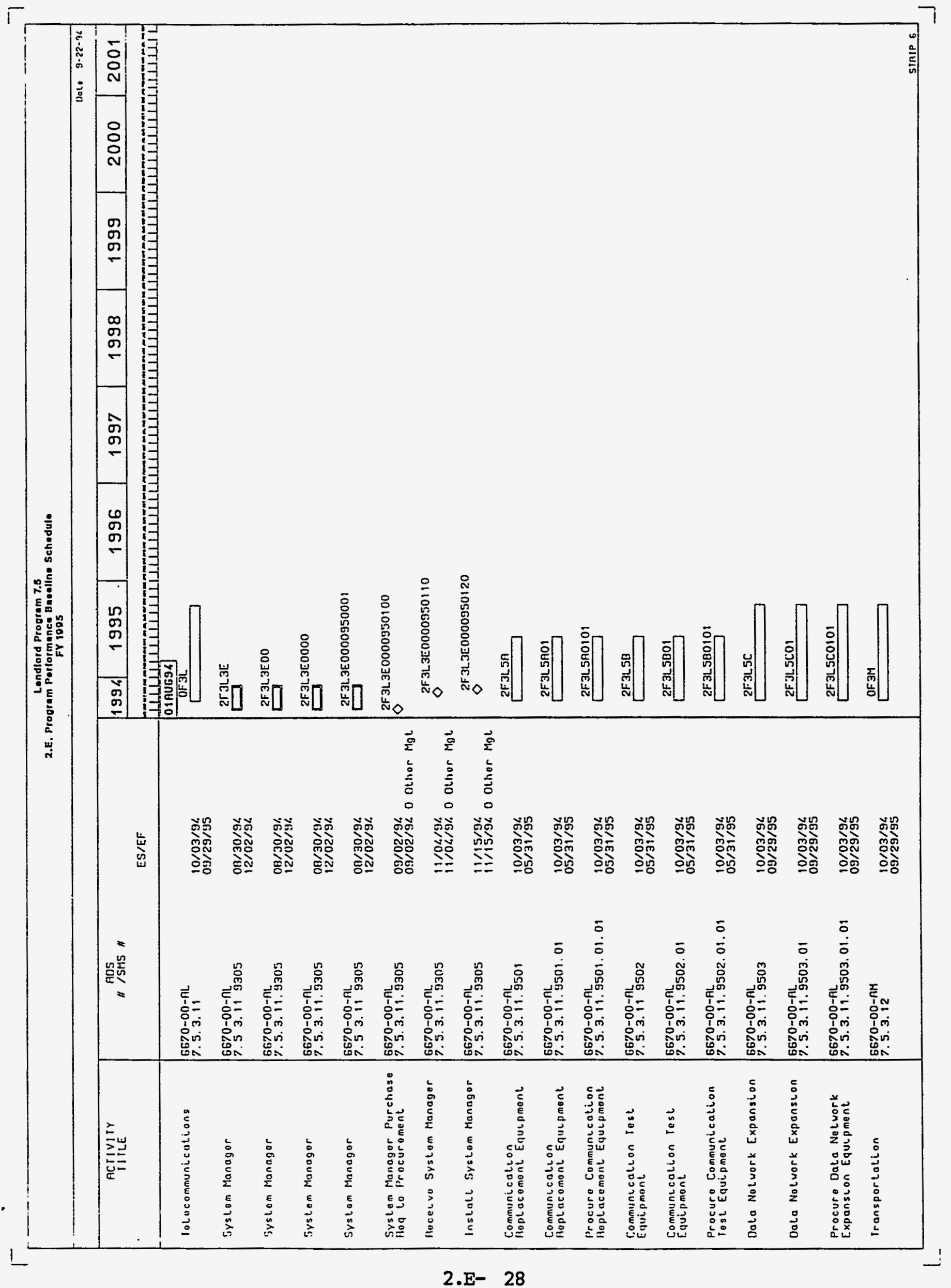




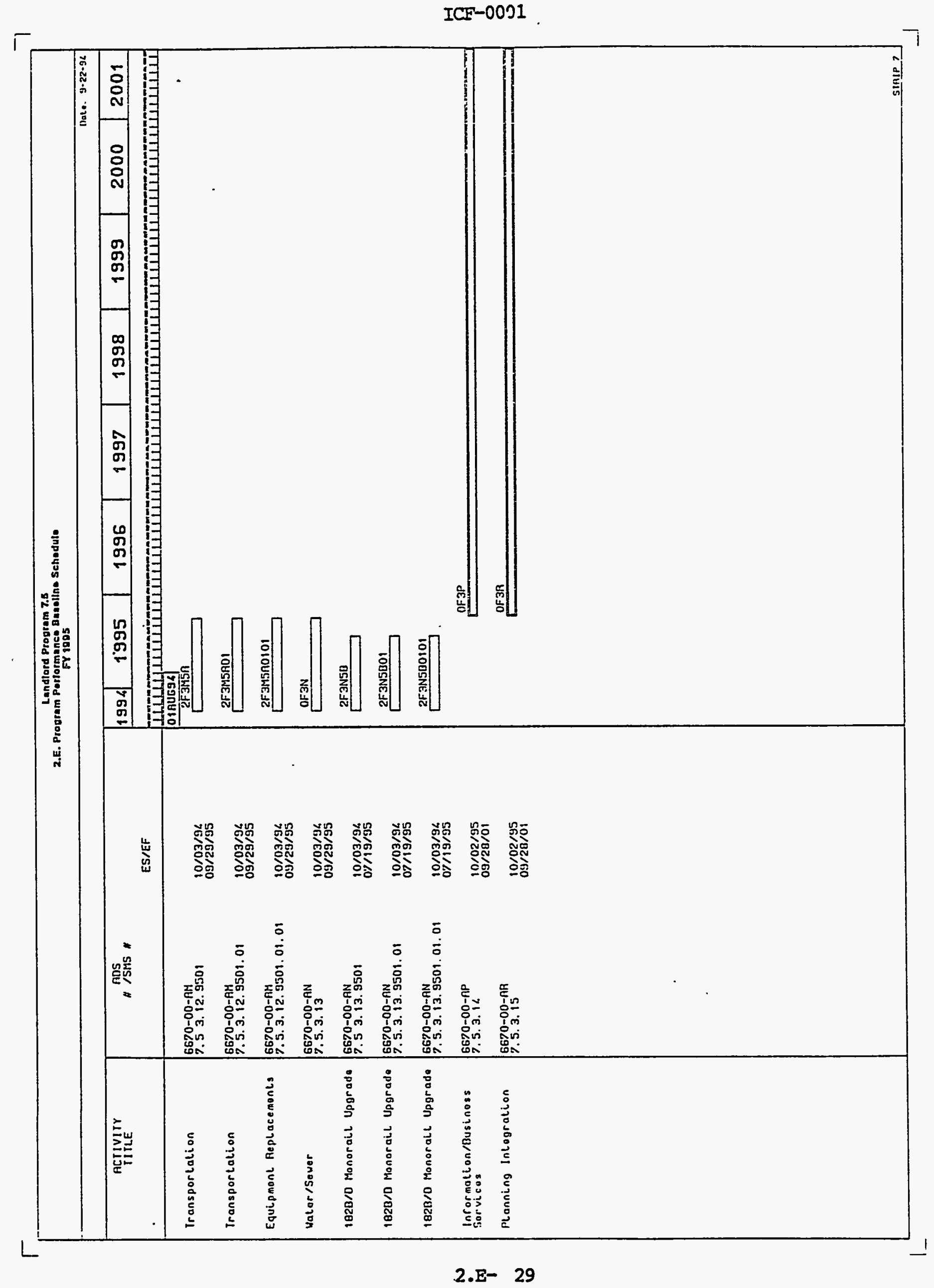




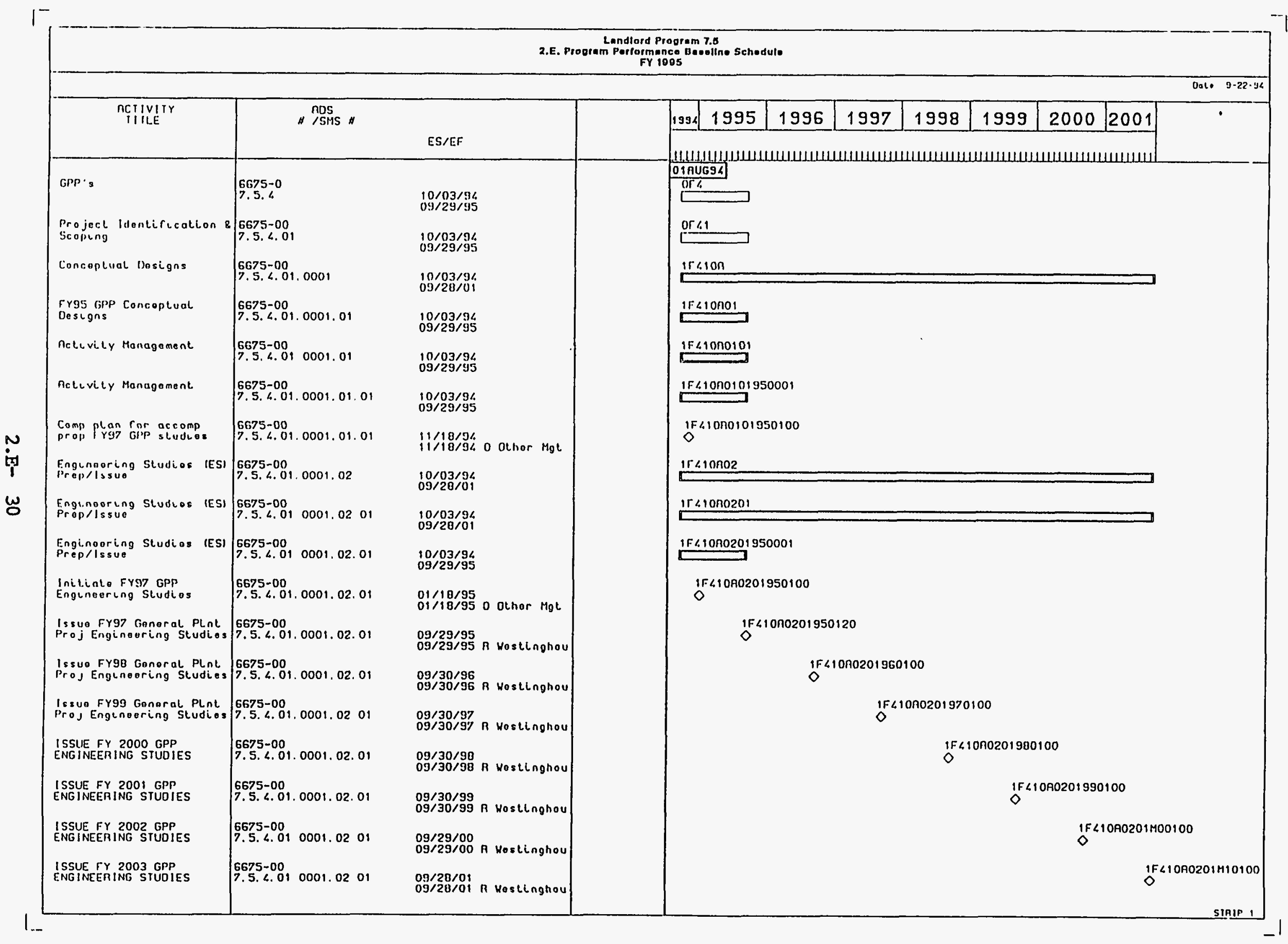




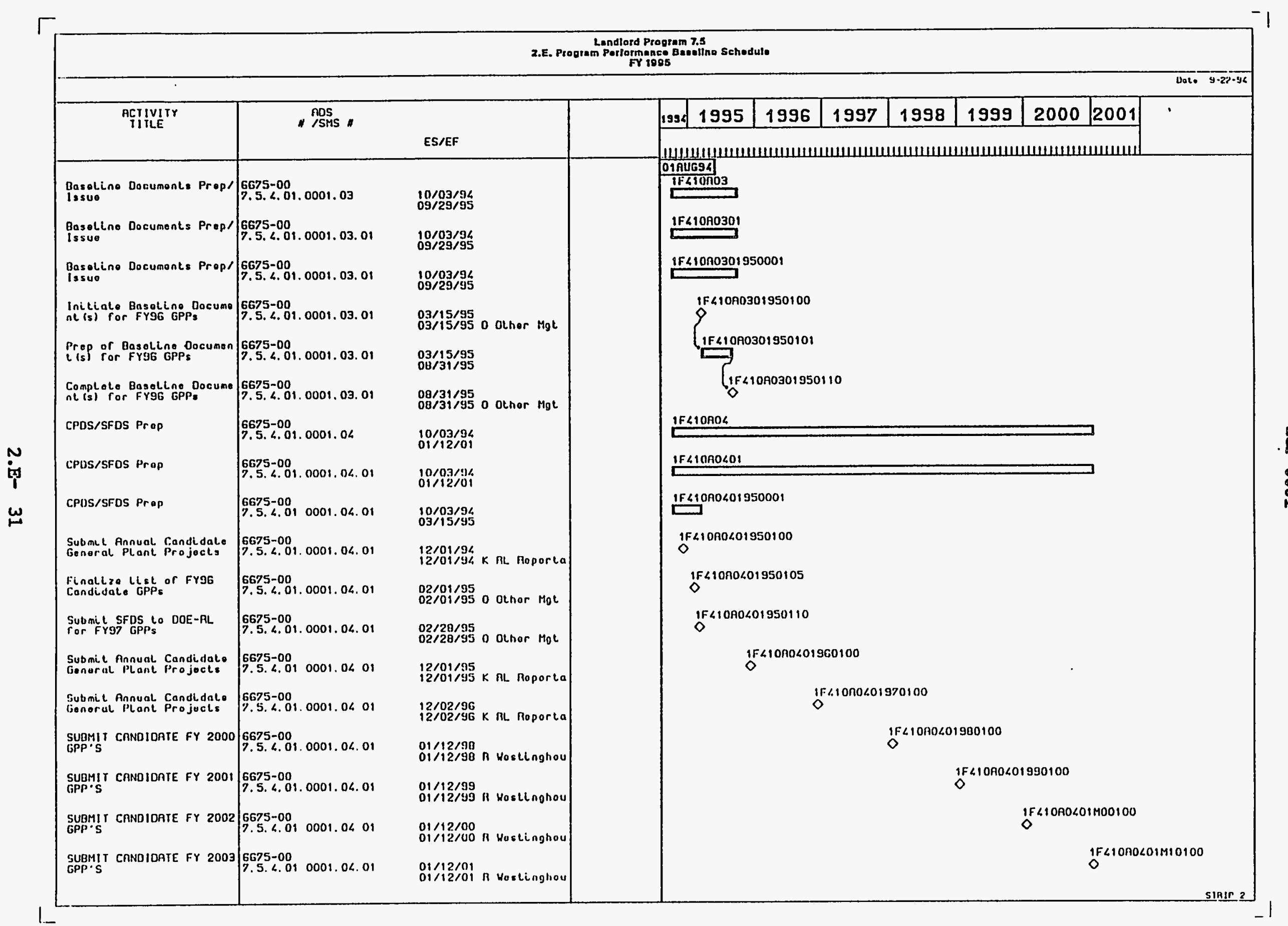




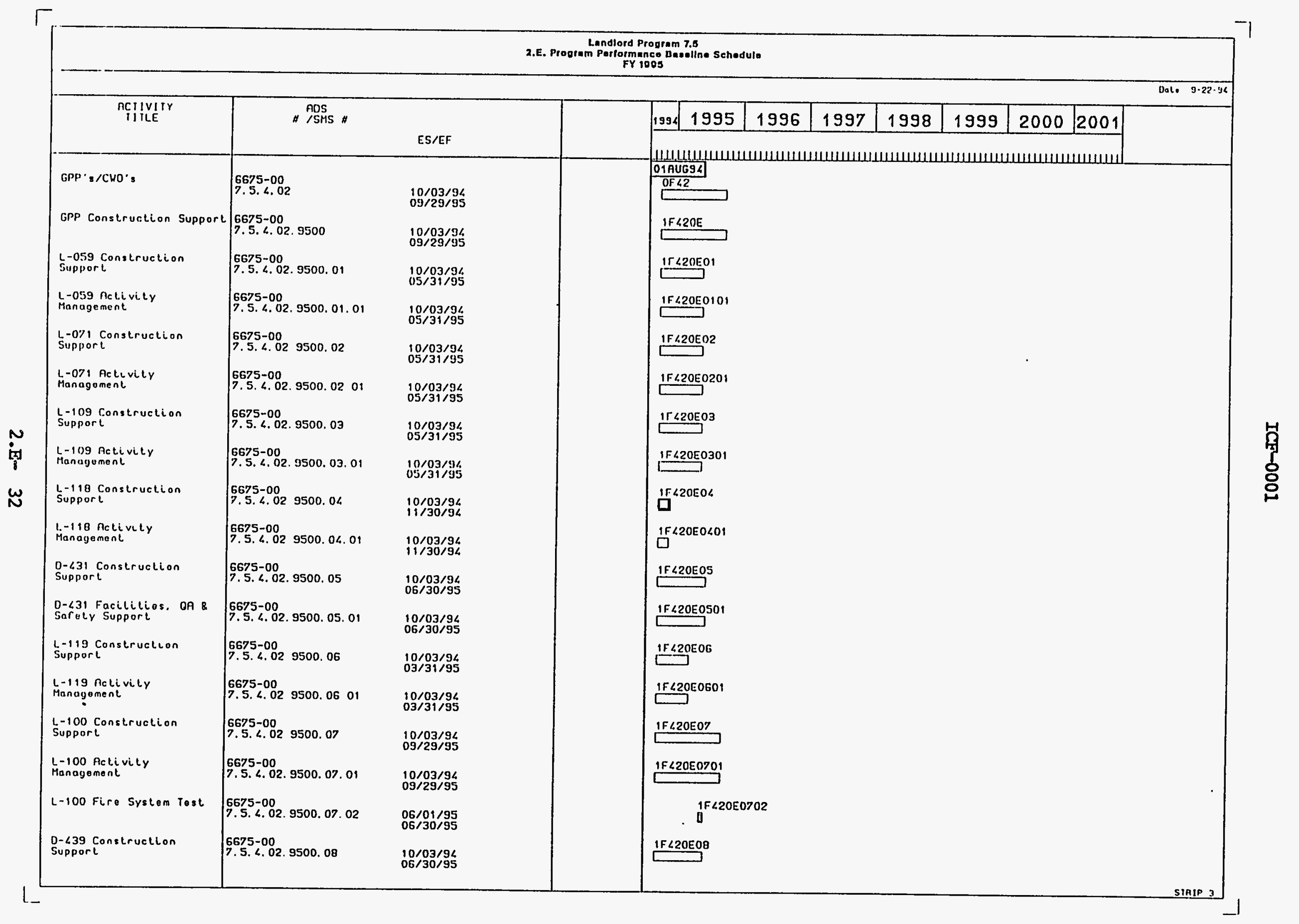




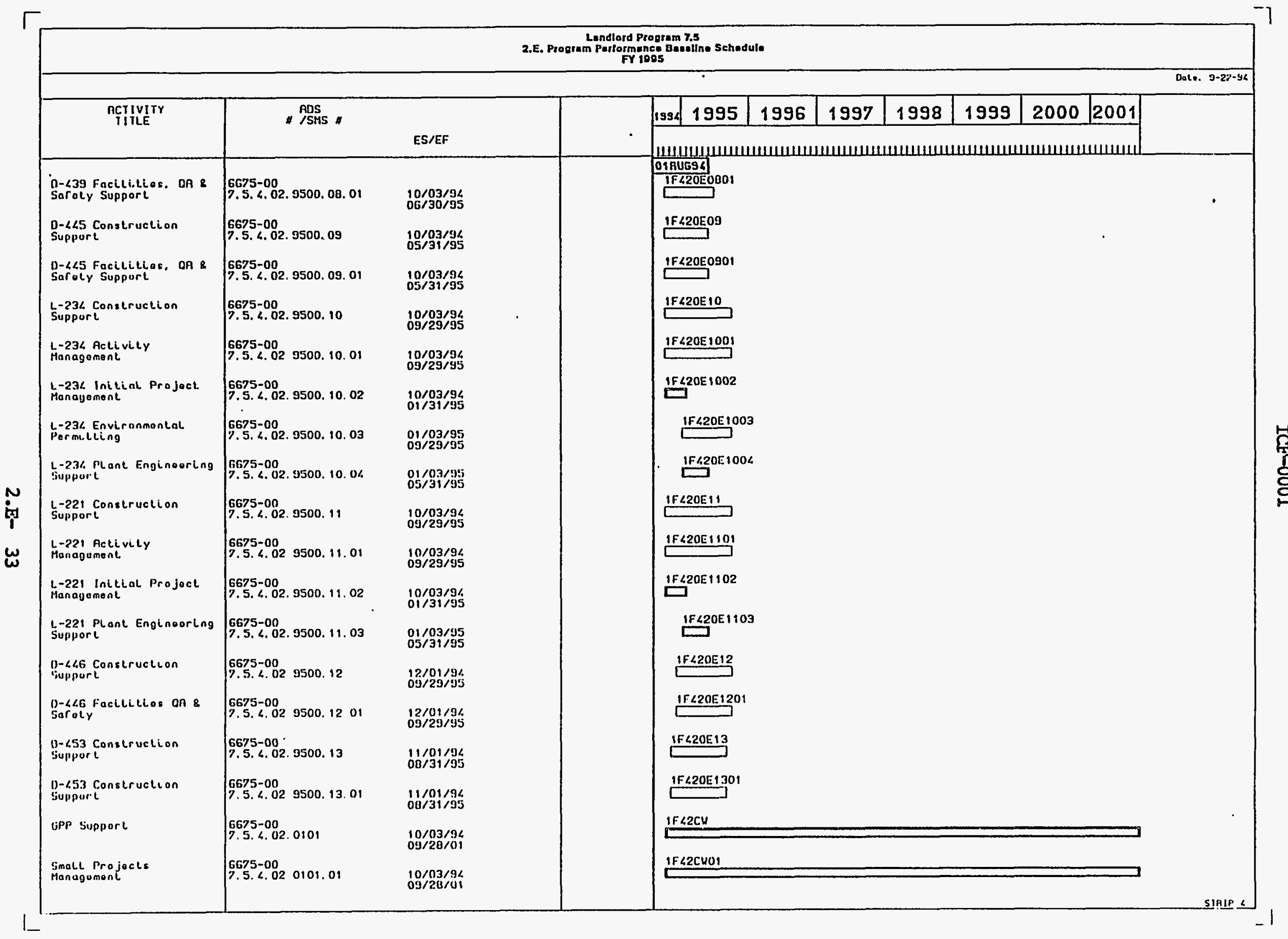




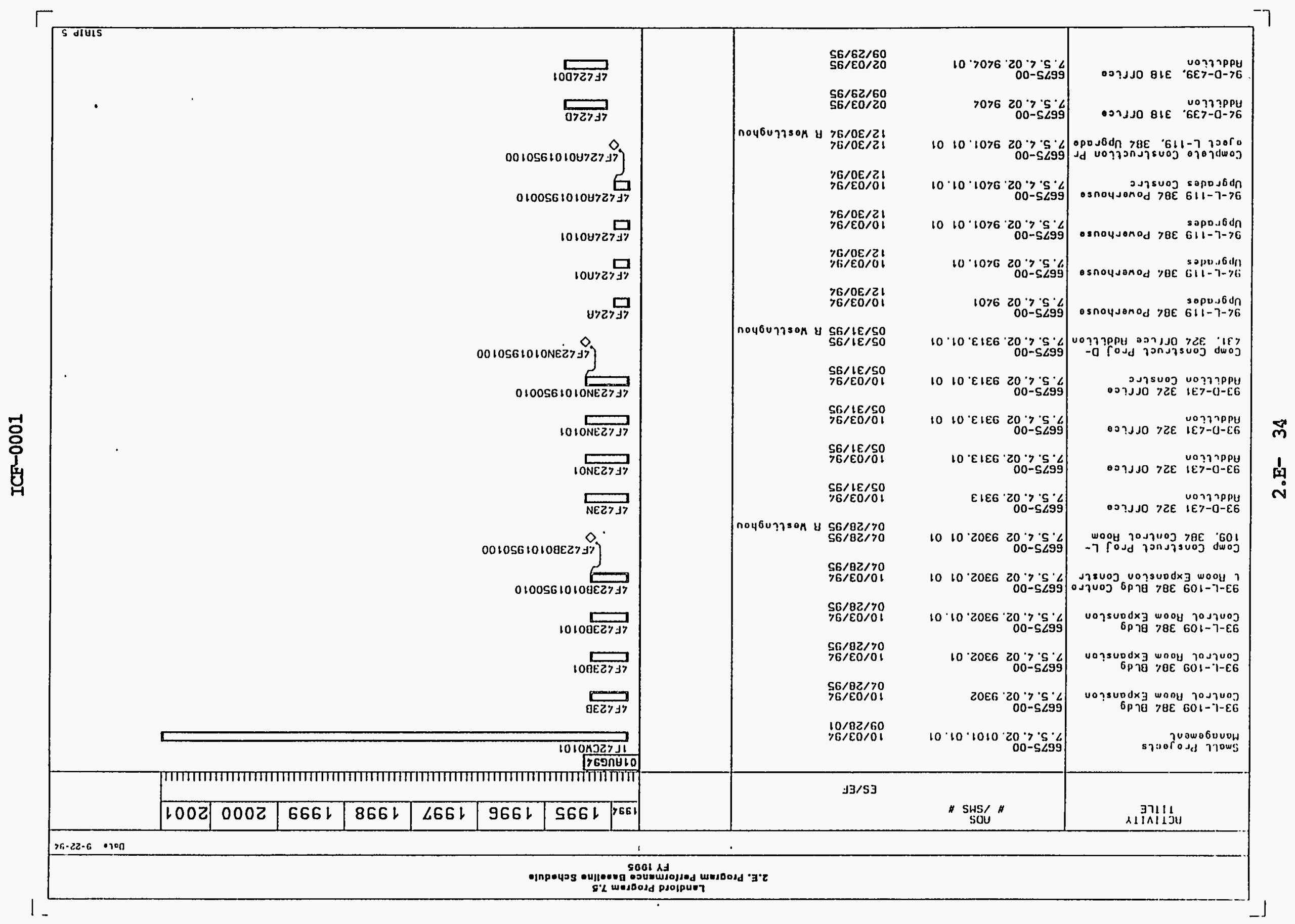




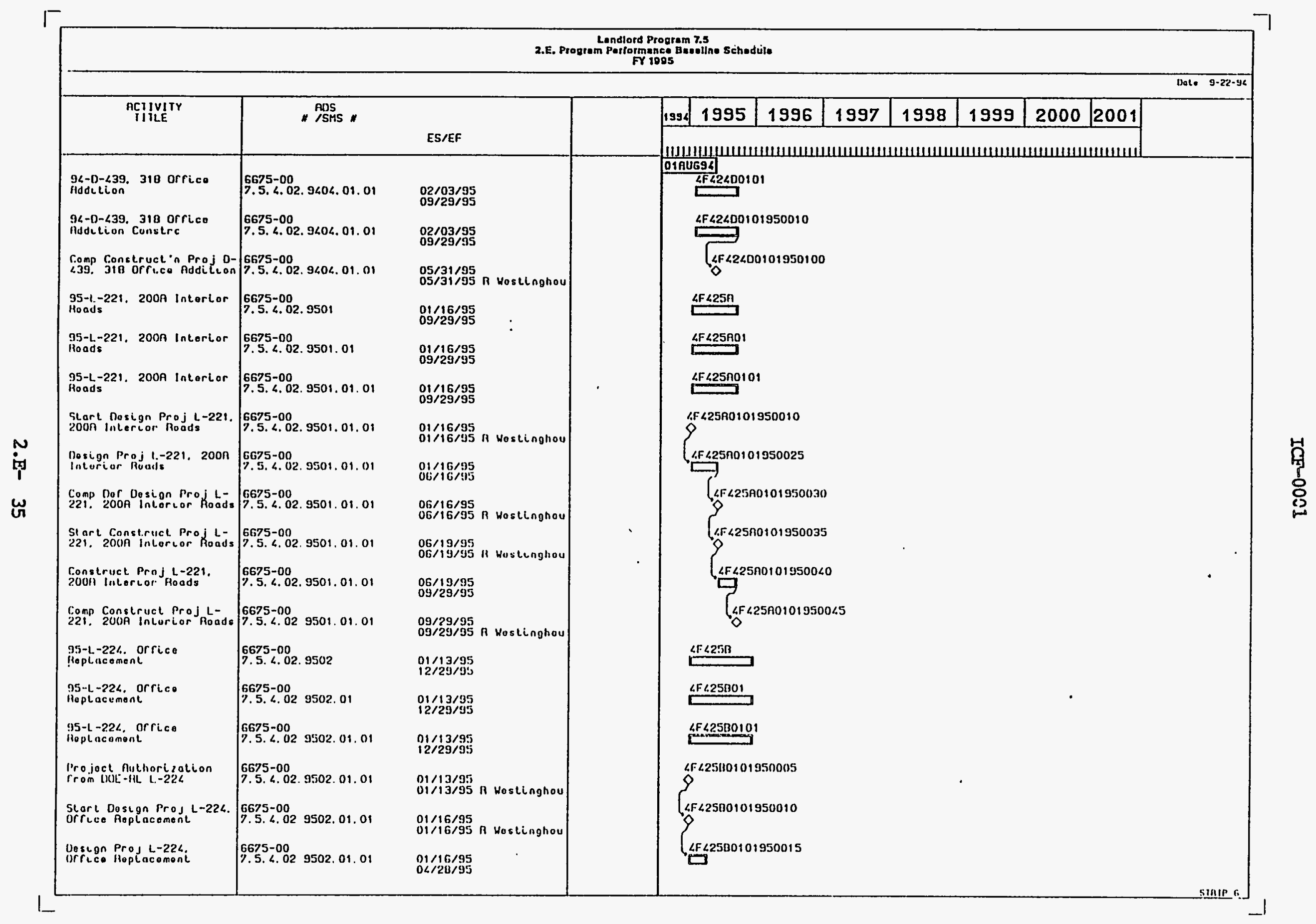




\begin{tabular}{|c|c|c|}
\hline$\substack{\text { ACTIVITY } \\
\text { IITLE }}$ & ADS \\
\hline
\end{tabular}

Comp Dosegn Pros L-224. $6675-00$
Orreco Hoplacomone

$04 / 28 / 95$
$04 / 28 / 95$ a Hostlinghou

$\begin{array}{lll}\text { Start Conslruct Proj L- } & \text { 6675-00 } \\ 226, \text { of fuce Heplacoment } & \text { 7.5.4.02.3502.01 }\end{array}$

Construct Proj L-22L.
ior reto Paplacomont

6675-00
7.5.4.02.9502.01.01

$05 / 01 / 95$
$05 / 01 / 95$ n Hostinghou

Comp Construcl Proj L-

$6675-00$
7.5.4.02.9502.01.01

95-L-234. LO0A Sanilary
Lagoon

95-L-234. LOOA Sanitary

$6675-00$

7.5.4.02. 3503.01

$05 / 01 / 95$

$12 / 29 / 95$
$12 / 29 / 95$ Nostinghou

$01 / 16 / 95$
$07 / 16 / 96$

95-L-234, 400 Sanilary

$6675-00$
7.5.4.02. 9503.0101

$\begin{array}{lll}\text { SLart Dosign Project L-2 } & 6675-00 \\ \text { 34. } 1.001 \text { Sant Lary Lagoon } & \text { 7.5. } 4.02 .9503 .01 .01\end{array}$

$\begin{array}{ll}\text { Wosign ProjocL L-234. } & 6675-00 \\ \text { LoOA SancLary Lagoon } & 7.5 .4 .02 .3503 .01 .0\end{array}$

Comp Dor Design Proj L-2 6675-00

Comp Dor Design Proj L-2
34. Loon Sanctary Lagoon

$\begin{array}{lll}\text { Start Construct Proj L-2 } & 6675-00 \\ \text { 34. LOOA Sanctary Lagoon } & \text { 7.5.4.02. } 5503.01 .01\end{array}$

Construet Proj t-23L. 6675-00

Construet Praj L-23L. 65675-00

Comp Construct Proj L-23 6675-00

95-D-446. 324 HLQhbay

Ardition

7. 5. 4. 02. 3503. 01.0

6675-00 92.9504

95-D-LL6. 32L Highbay
Mddulion.

7675-00 9504.01

95-D-L46, 324 Highbay

nddition

$6675-00$
$7.5 .4 .02 .950<.01 .01$

Start Dostgn $95-0-466$.
322 Milihbay Addition

$6675-00$
7.5 .4 .02 .9504 .01 .01

Dosign Proj D-<L6. 326

$6675-00$
$7.5 .4 .02 .950<.01 .01$

\begin{tabular}{|l|l|}
\hline 994 & 1995 \\
\hline
\end{tabular}

1996

1997

1998

1999

\begin{tabular}{|l|l|}
2000 & 2001 \\
\hline
\end{tabular}

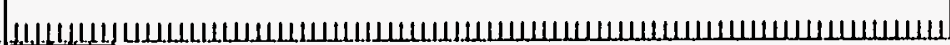
01 AUG95

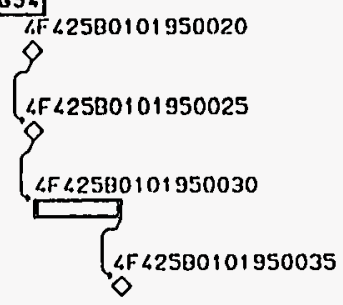

$01 / 16 / 95$
$07 / 16 / 96$

$01 / 16 / 95$
$07 / 16 / 96$

$01 / 16 / 95$
$01 / 16 / 95$ nostinghow

$01 / 16 / 95$
$06 / 30 / 95$

$06 / 30 / 95$

66/30/95 n Nostingliou

$07 / 05 / 95$

R Yostlonghou

$07 / 05 / 95$

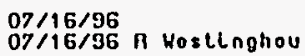

$01 / 16 / 95$

$01 / 16 / 95$
$01 / 31 / 96$

$01 / 16 / 95$
$01 / 31 / 96$

01/16/95 R Hestinghou

01/16/95 


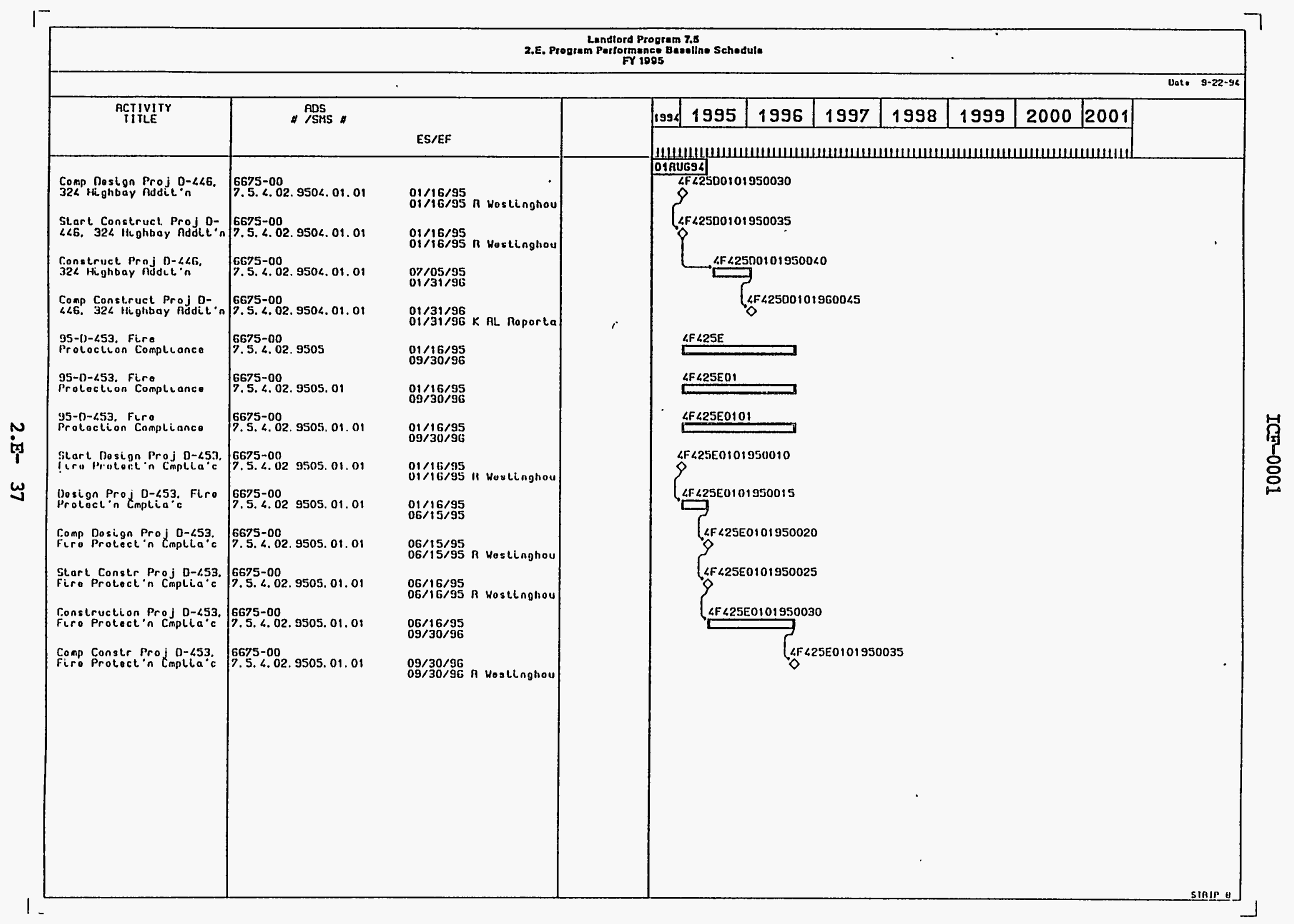




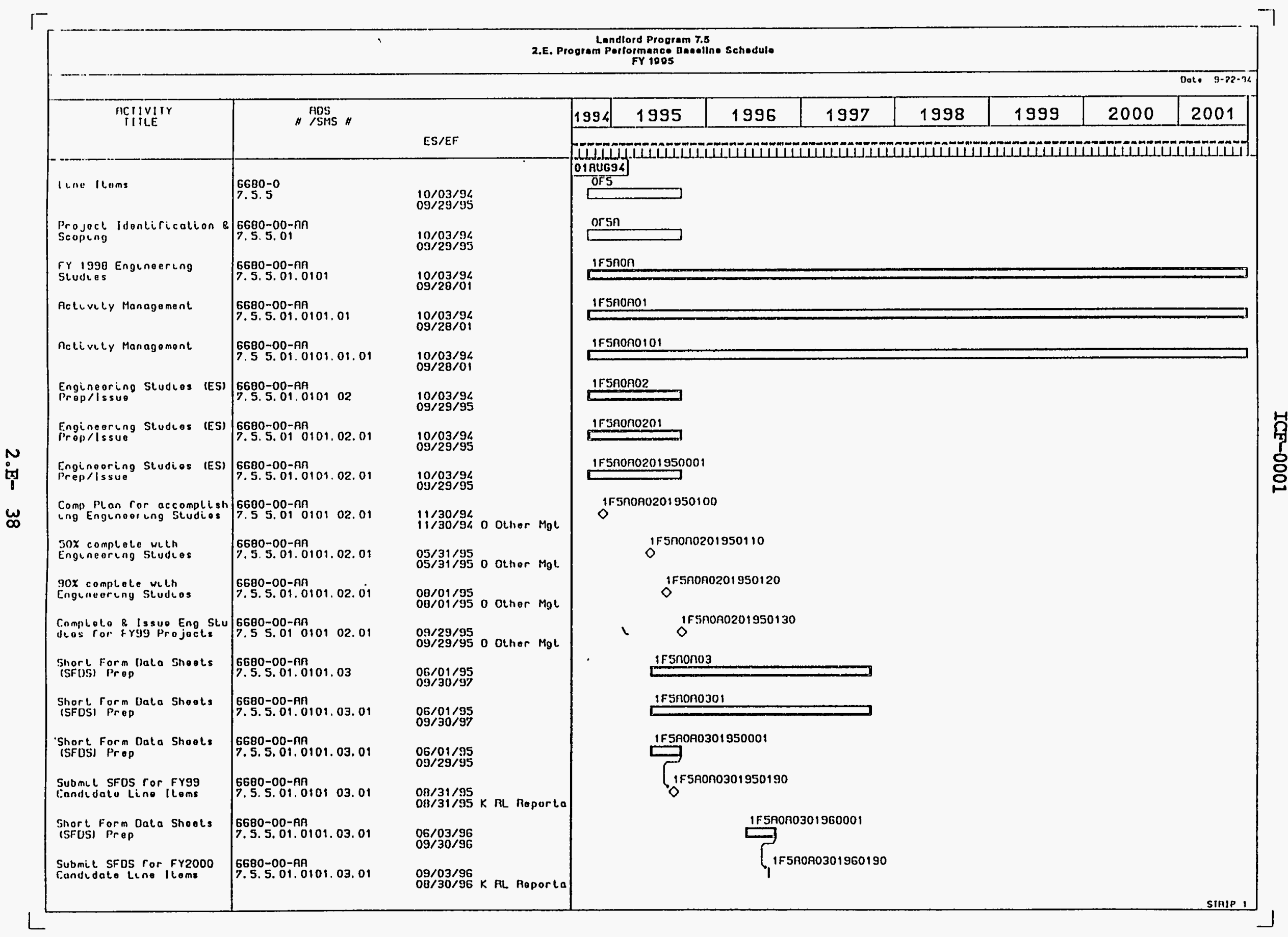




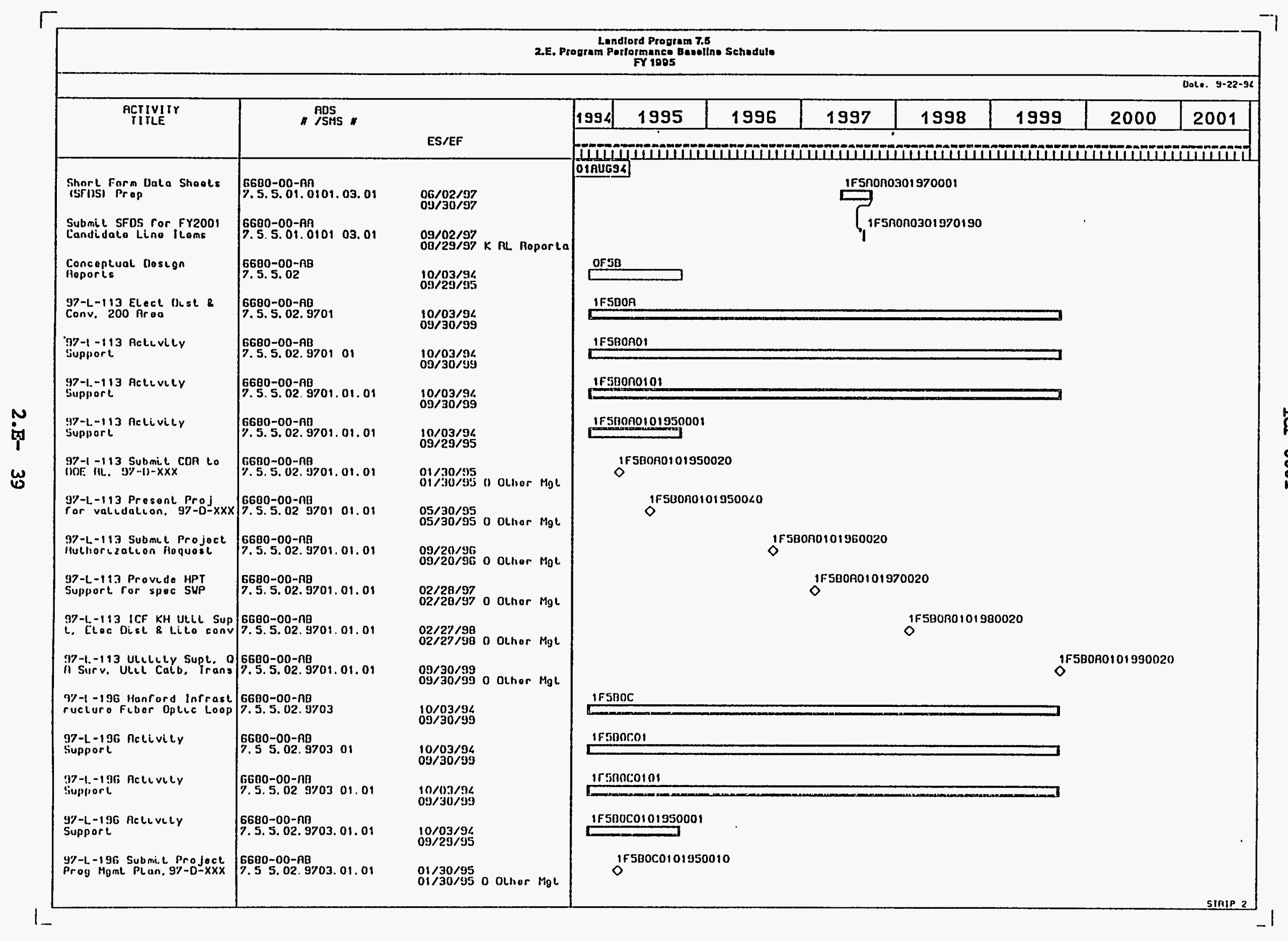




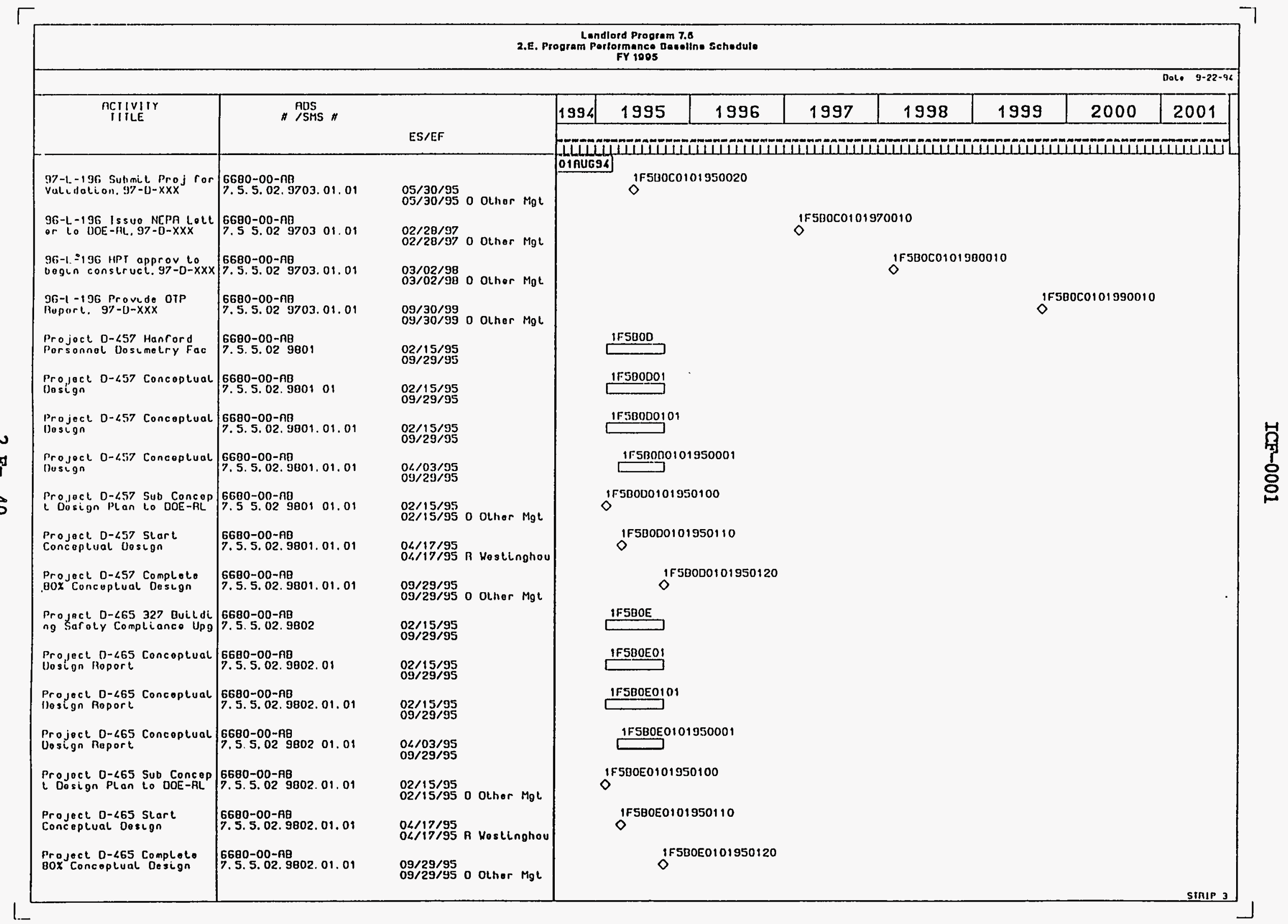




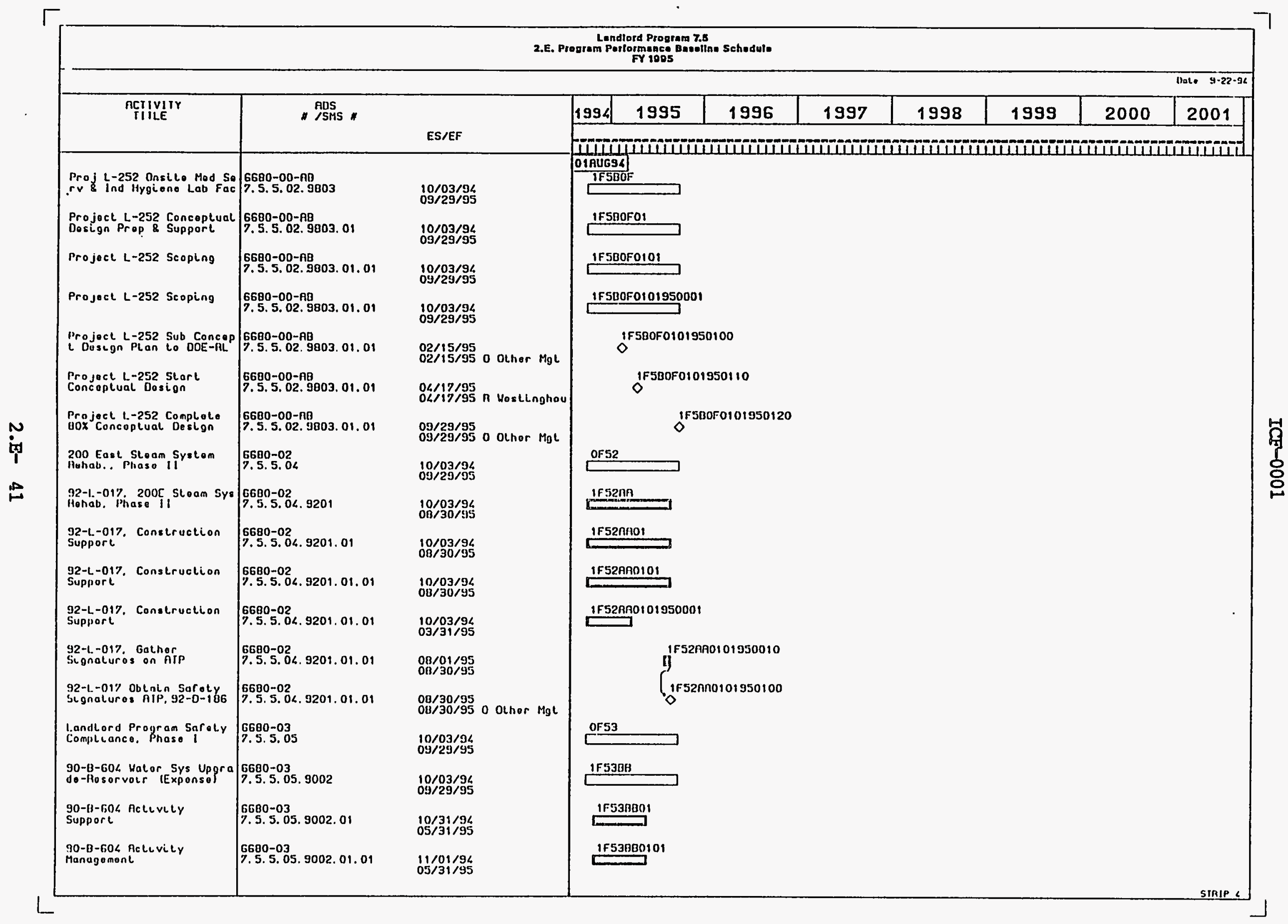




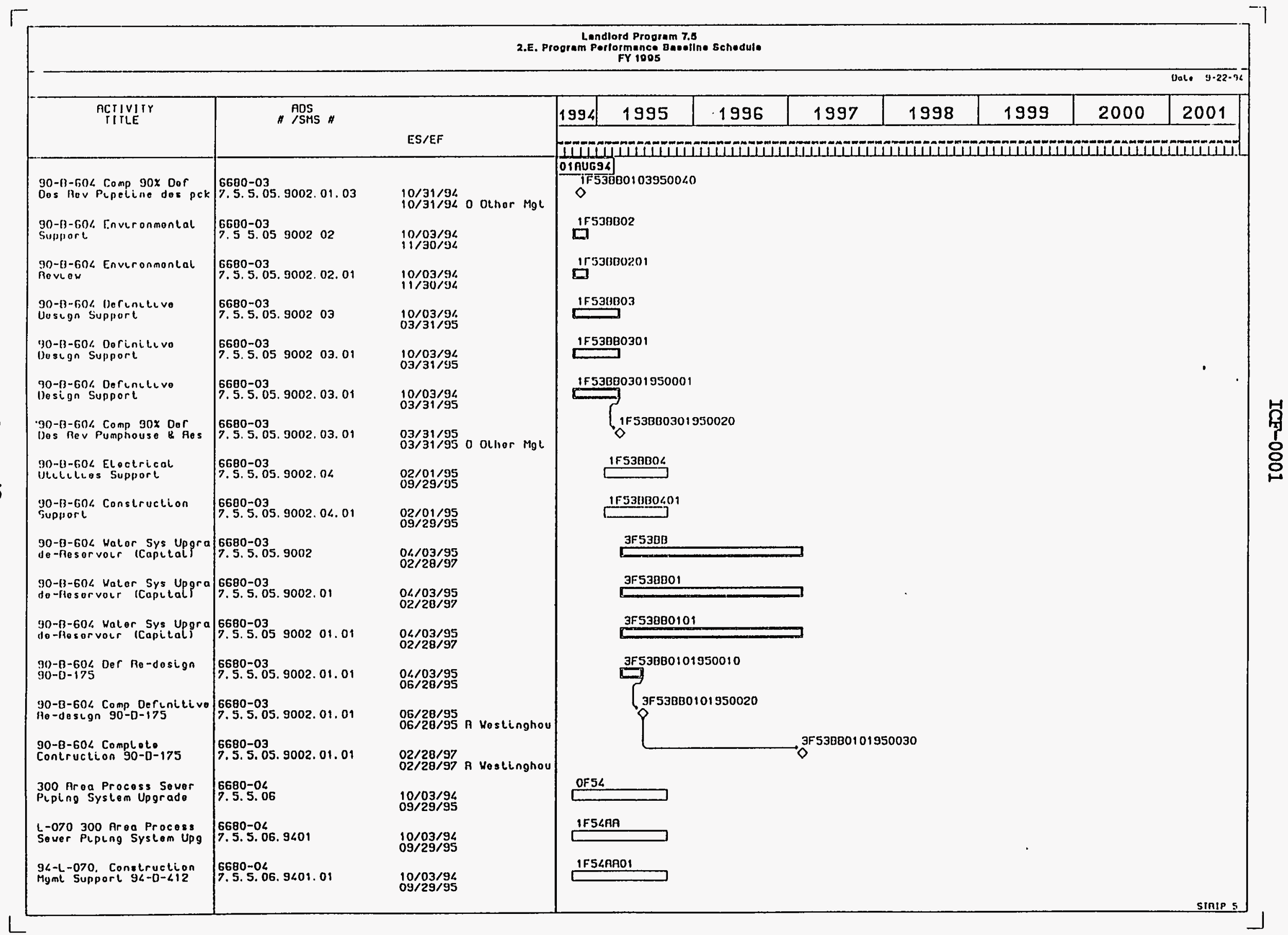




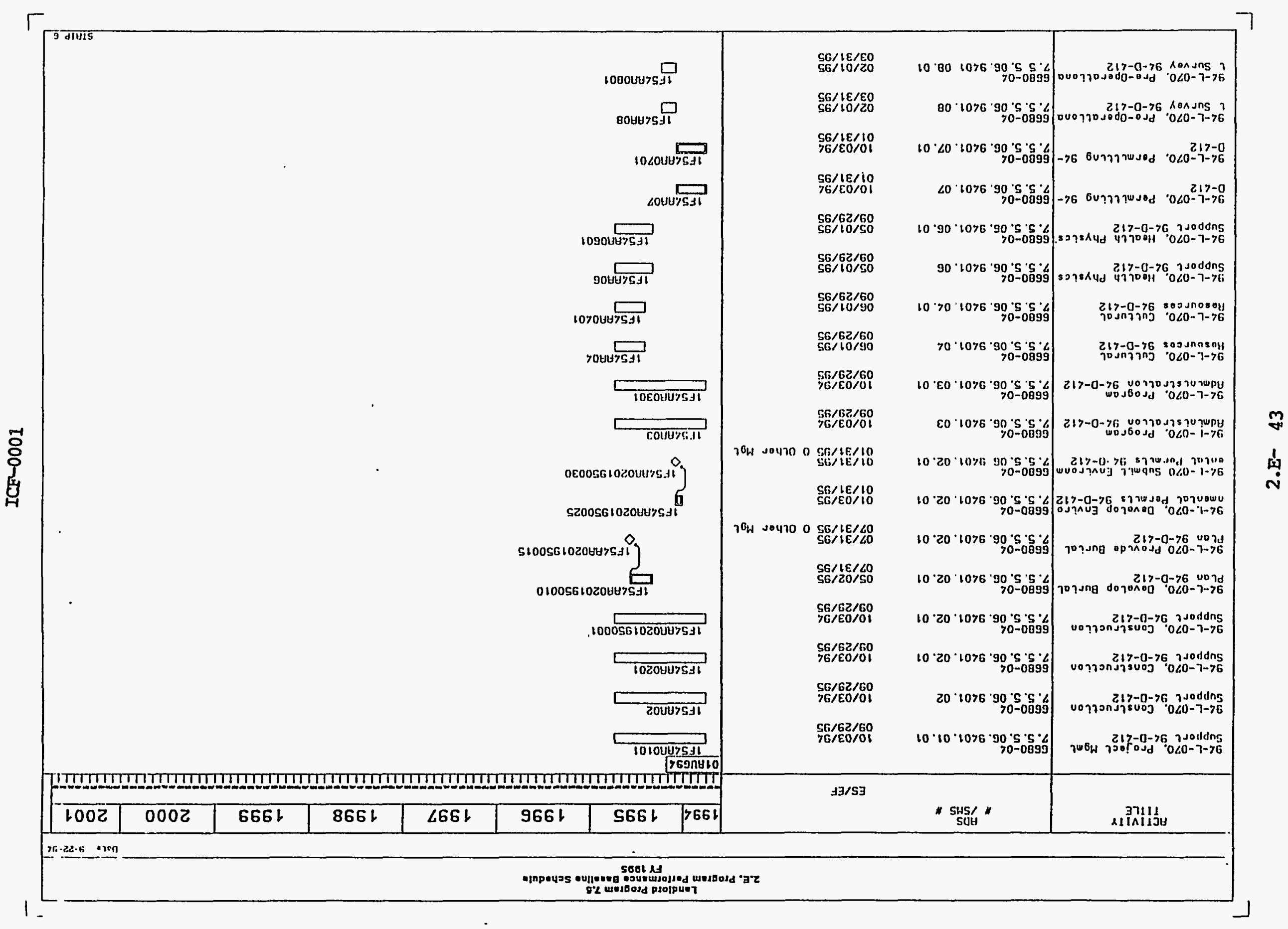




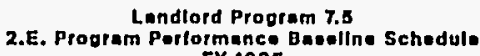

FY 1005

\begin{tabular}{|c|c|c|c|c|c|c|c|c|c|}
\hline & & & & & & & & & Dute $9-22-36$ \\
\hline \multirow[t]{2}{*}{ ACIIVIIY } & \multirow[t]{2}{*}{ nos $\stackrel{\text { nSMS }}{*}$} & 1994 & 1995 & 1996 & 1997 & 1998 & 1999 & 2000 & 2001 \\
\hline & & \multicolumn{8}{|c|}{ - } \\
\hline
\end{tabular}

94.-L.-070 Propare Pre-0p
Enviro noqs $9 \%-(0-612$

$9 \angle-L-070$. Propare Pre-Op
Fnviro Meqs $94-0-412$

$6680-04$
7.55 .06940108 .01

6600-04 9406.9401 .08 .0

$02 / 01 / 95$

olher MoL

300 Aroa Procoss Sover

$6680-04$
$7.5 .5,06.940$

$02 / 01 / 95$
$03 / 31 / 95$

300 Aroa Procosss Sever

6680-04

6680-04 9401.01

$10 / 03 / 94$

Poping syocon Upravor

PLping System Upgrado

9L-L-070 Camplele Derin

ULve Deslgn 9L-0-412

$6680-04$
$7.5 .5 .06 \quad 9401 \quad 01.01$

6680-02 9.5 5. 06. 9<01.02.01

96.-L-070 Submit Dosign
Documantation M-17-06.J

$34-L-070$ Slart

6680-0. 969.01 .02 .0

6680-04

5.5. 05940102.01

3L-L-070 SLarl Propare

6600-04

7.5.5.06. 9401.02.01

9L-L-070. Prepare ATP/

6680-06. 9401.02 .01

$6680-04$

$94-L-070$ Complele Cullur
aL Resourcos $9 \angle-0-\angle 12$

$94-\mathrm{L}-070$ Complet.

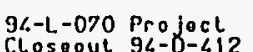

$6680-04$

5. 5, 06, 9401,02.01.

6680-04. 74.561 .02 .01

GL-LL-070 CompLets ATP/
OIP PLan $9<-D-612$

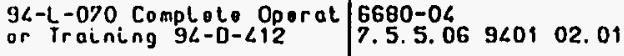

9L-L-070 Replace Process

6680-04 96.54 .06 .02 .01

Hancord Infrascruclura

$6680-05$

Underground Storago Iniks

32-L-0<4 Hanford Infragt

$6680-05$
7.5 .5 .07 .9201

$10 / 03 / 91$
$09 / 29 / 95$

$10 / 03 / 94$

$09 / 29 / 35$

$02 / 01 / 95$

95 K RL Roporta

04/28/35 T TrL-Parly

02/28/95 K RL Hoporta

$06 / 28 / 96$

$06 / 28 / 96$ a Hosllinghou

$07 / 01 / 96$
$06 / 30 / 97$

09/30/96 $09 / 30 / 96$ \& Westlughou

10/01/96 $10 / 01 / 96$ RL Roporla

12/31/96 \& vostinghou

$06 / 30 / 97$

97 n Nosllnghou

$06 / 30 / 97$

a voslinghou

$06 / 30 / 97$

I Tri-Party

$10 / 03 / 94$
$09 / 29 / 95$

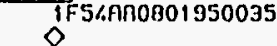

If54nn0001950040

3F $54 ., \mathrm{nn}$

3F54AnO

3 54AnO1

3F5LAnO101

3F54An0201350020

8

3FSLAnO201950021

3F54กA02019500L5

3rsunaoror 


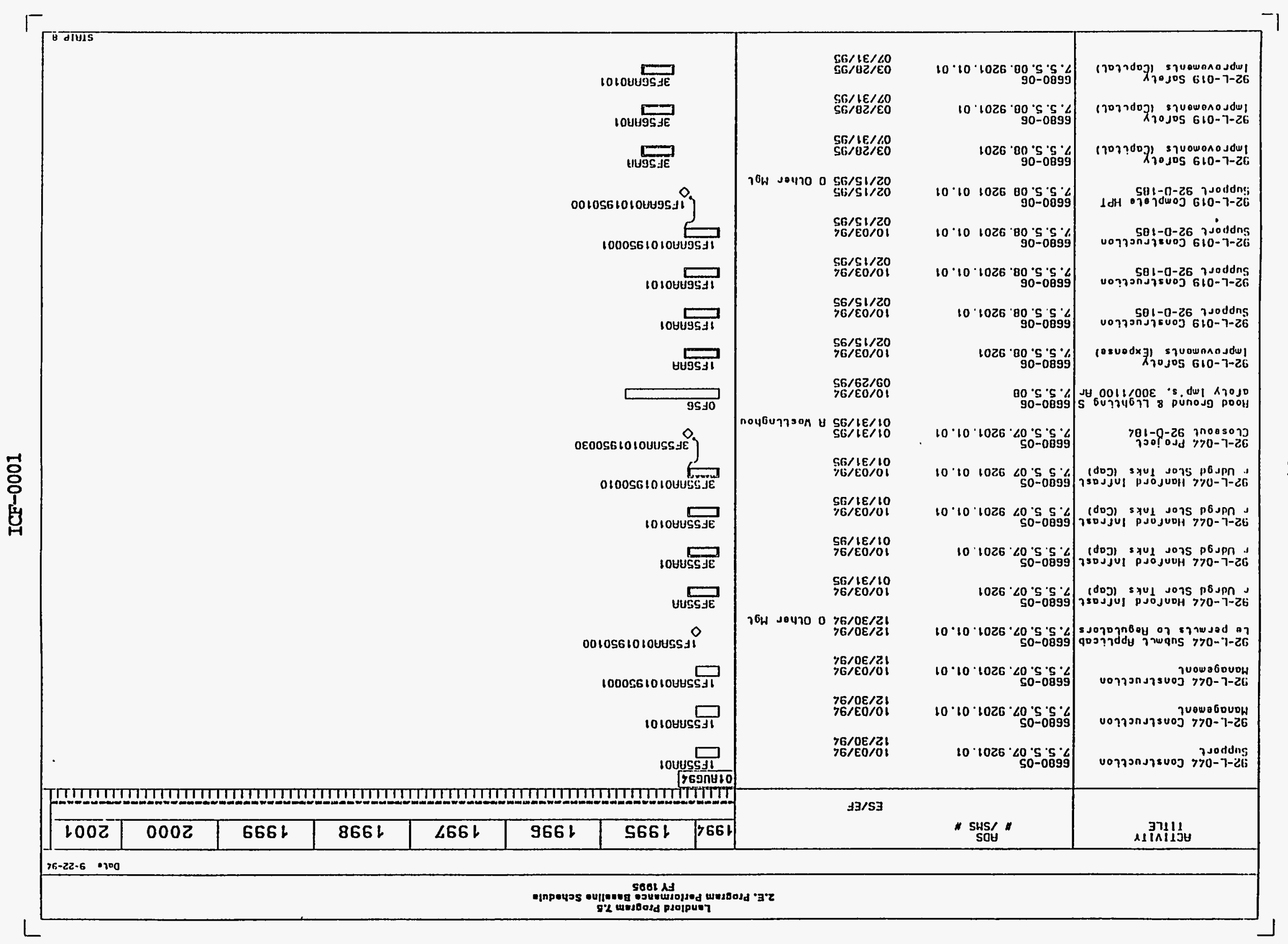




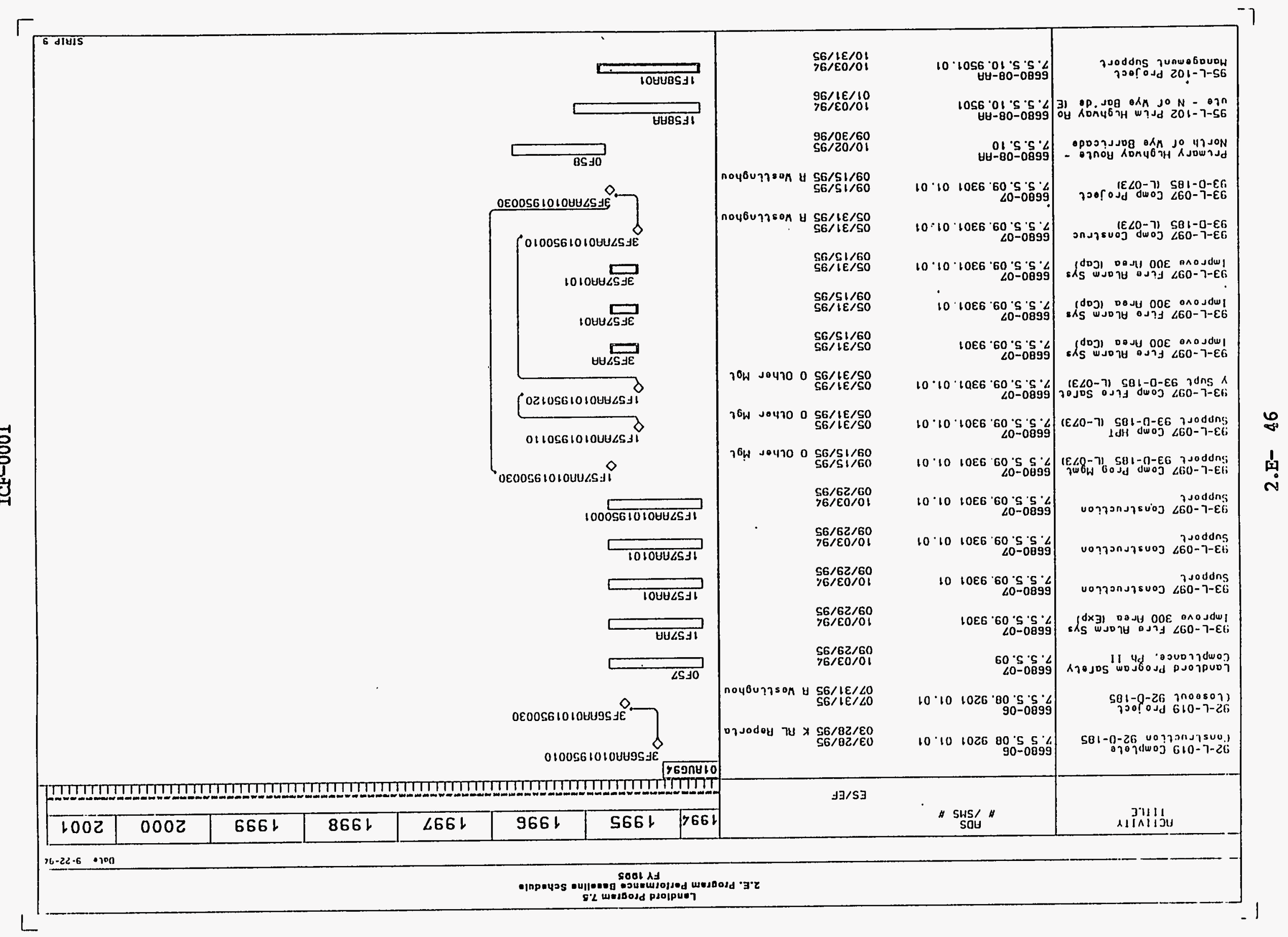




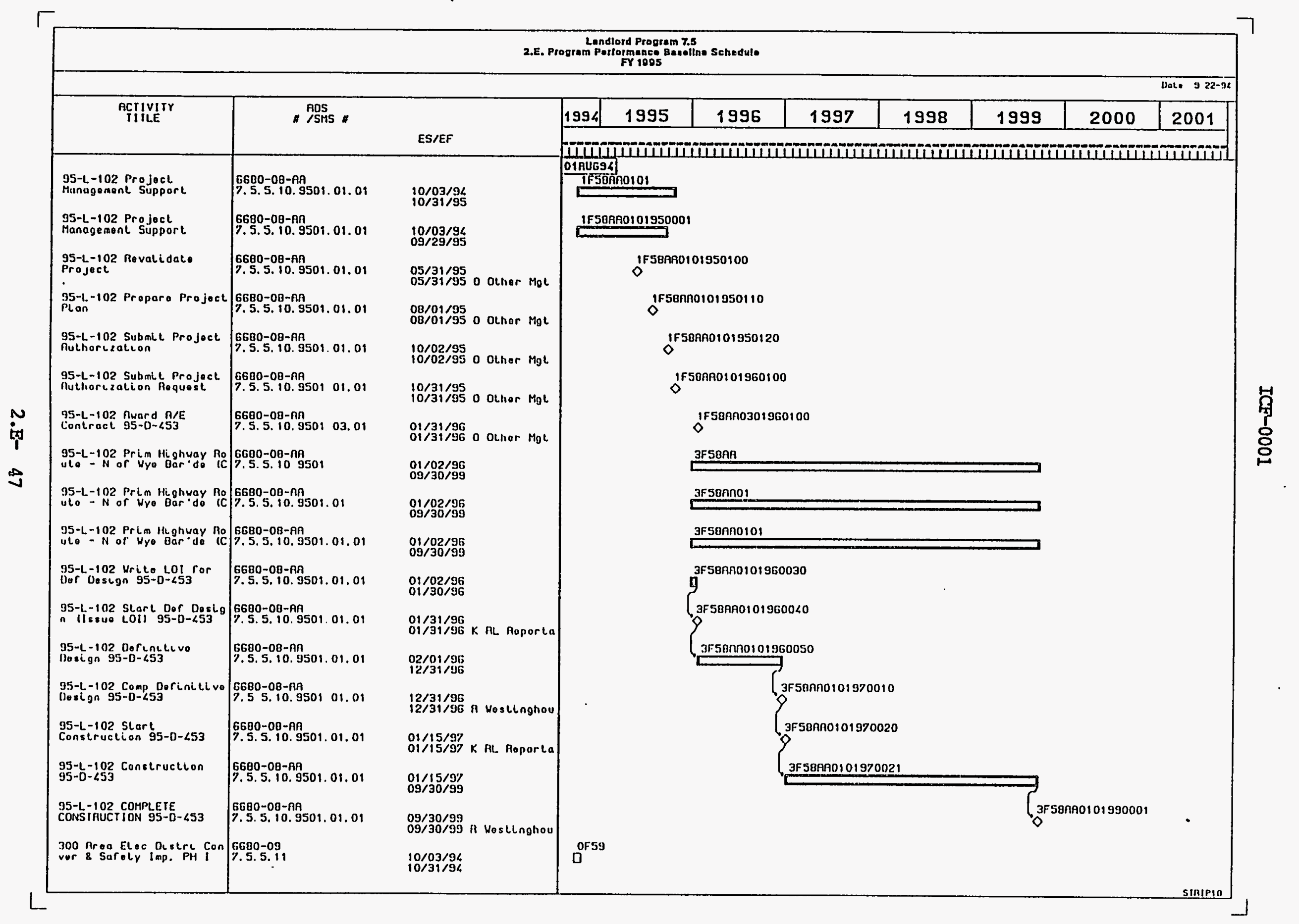




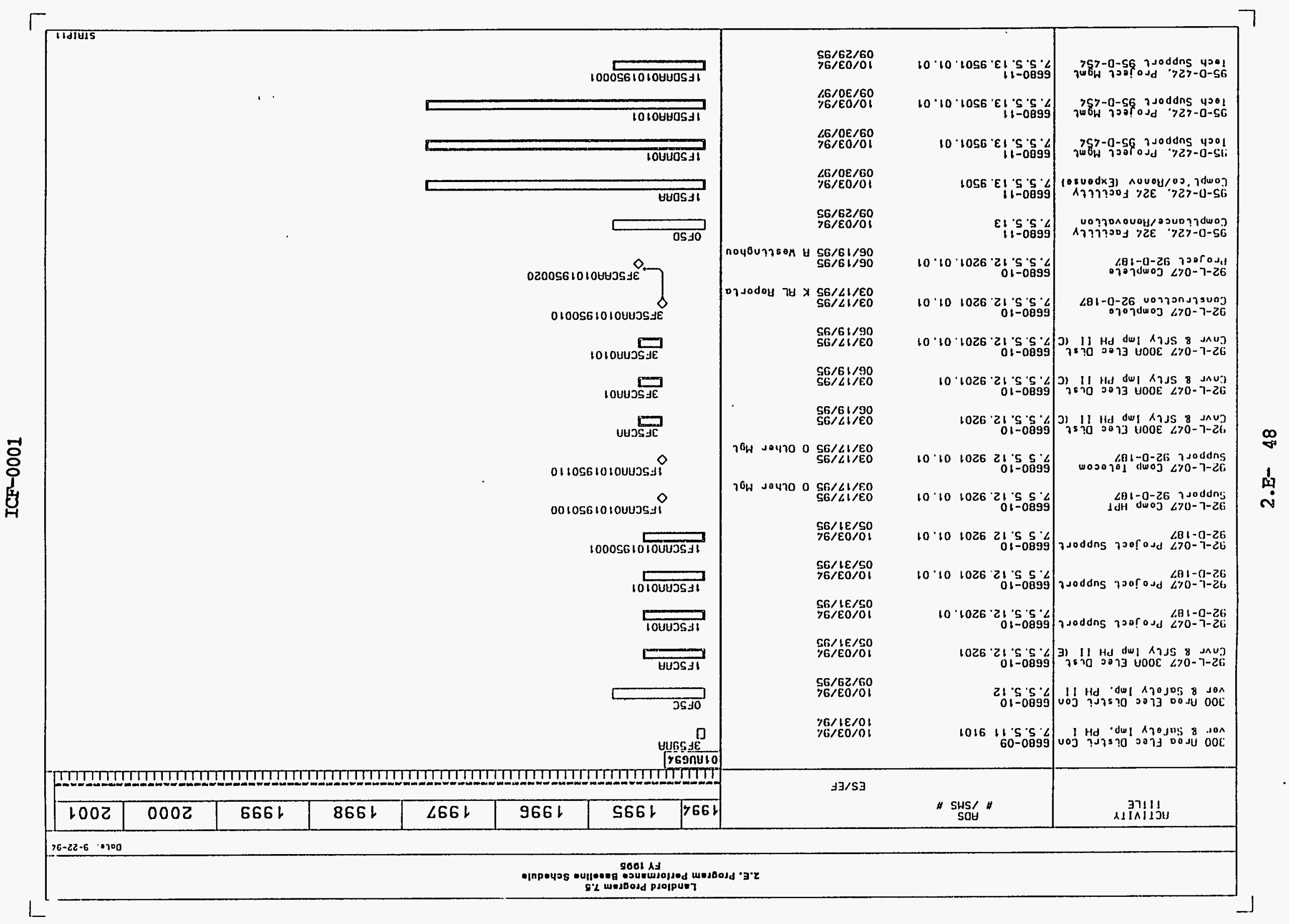




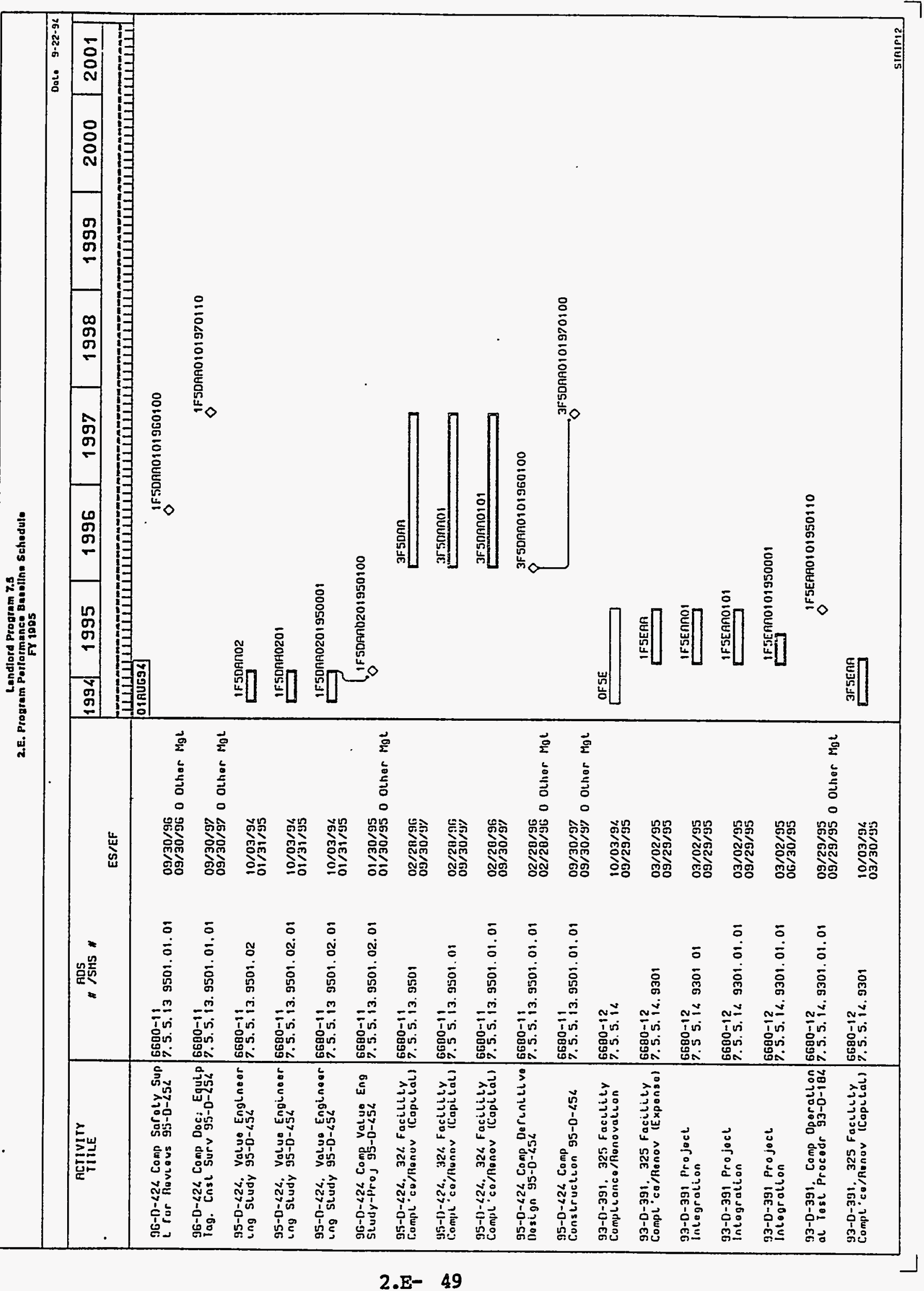




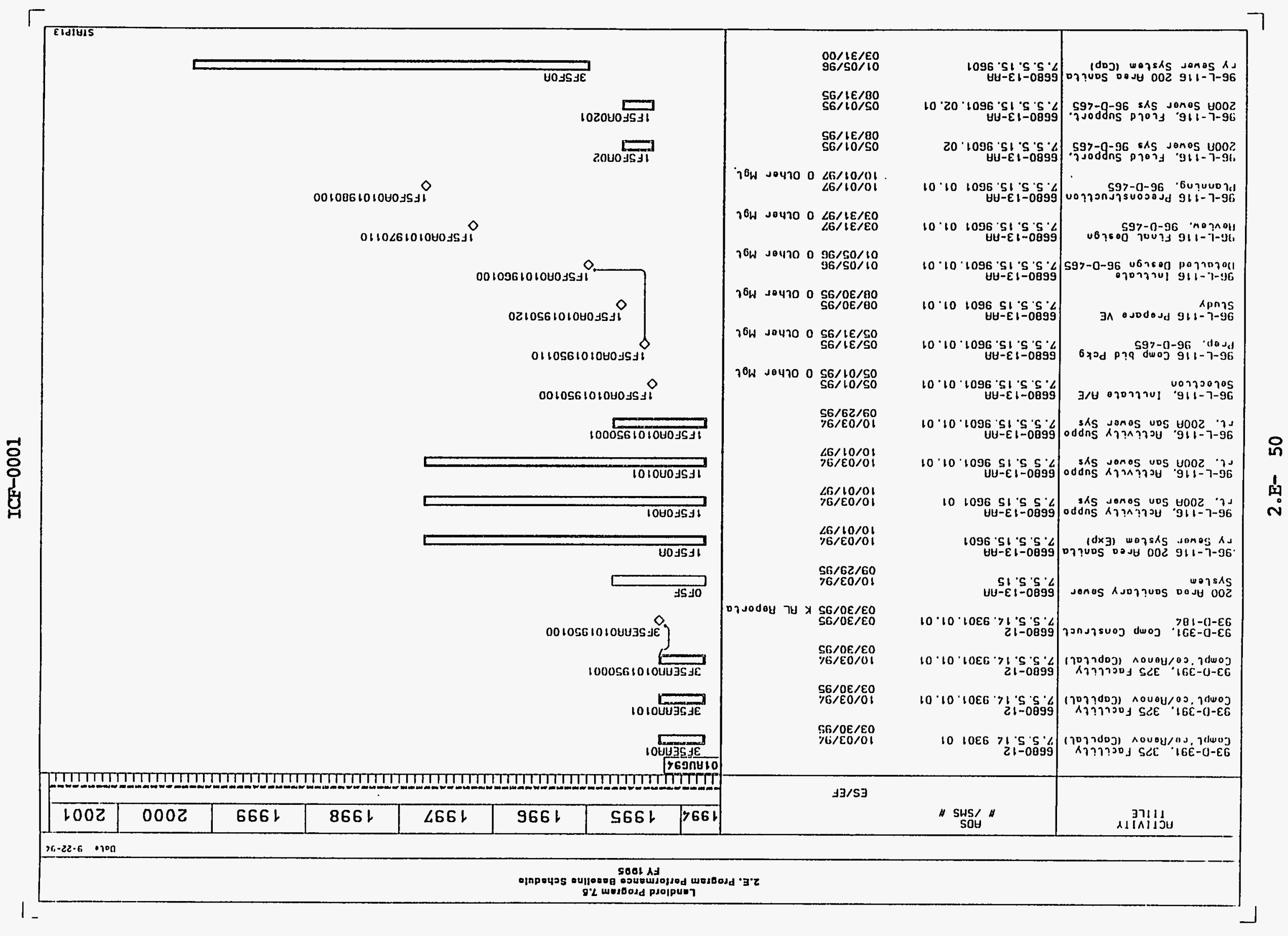




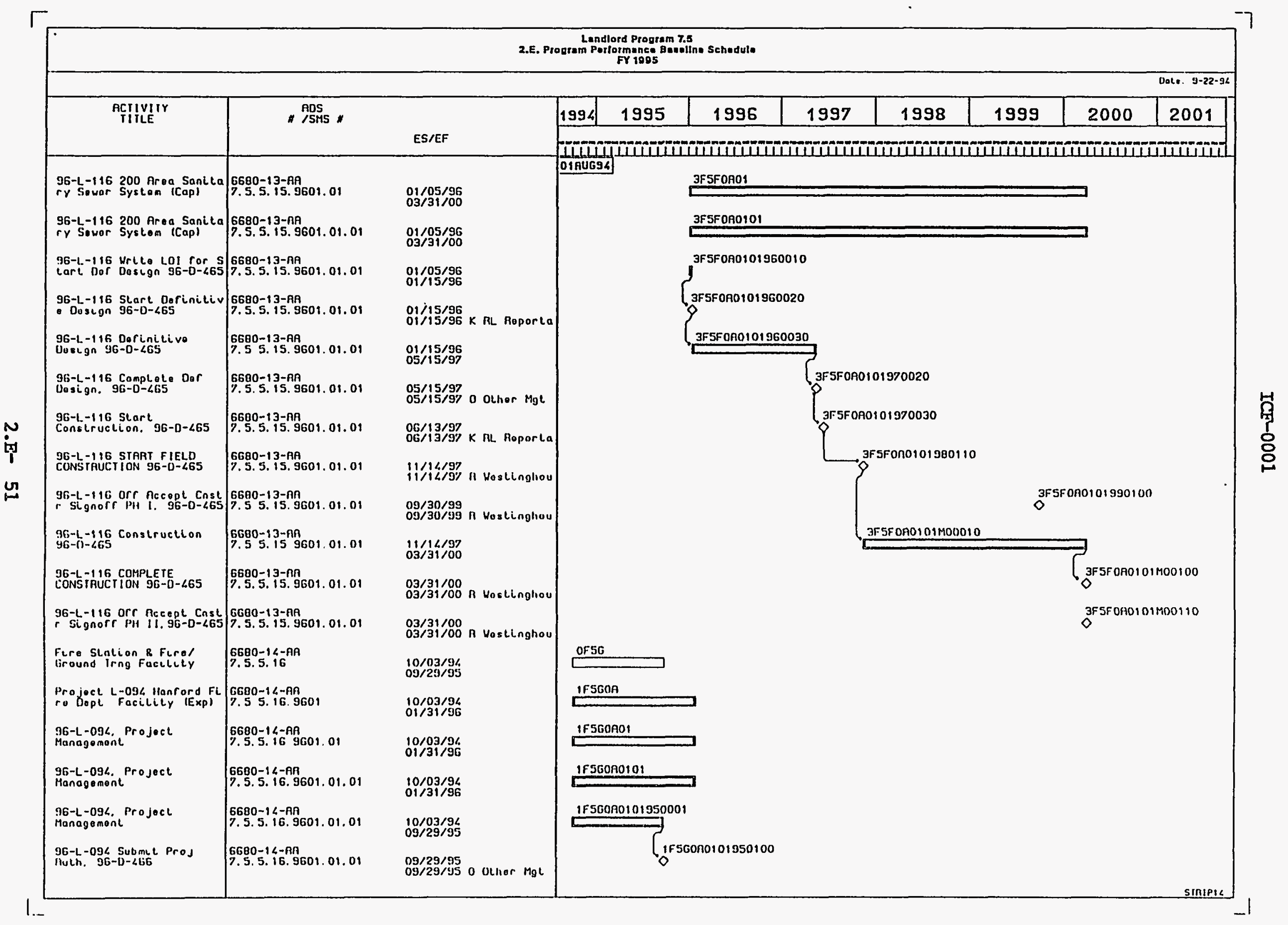




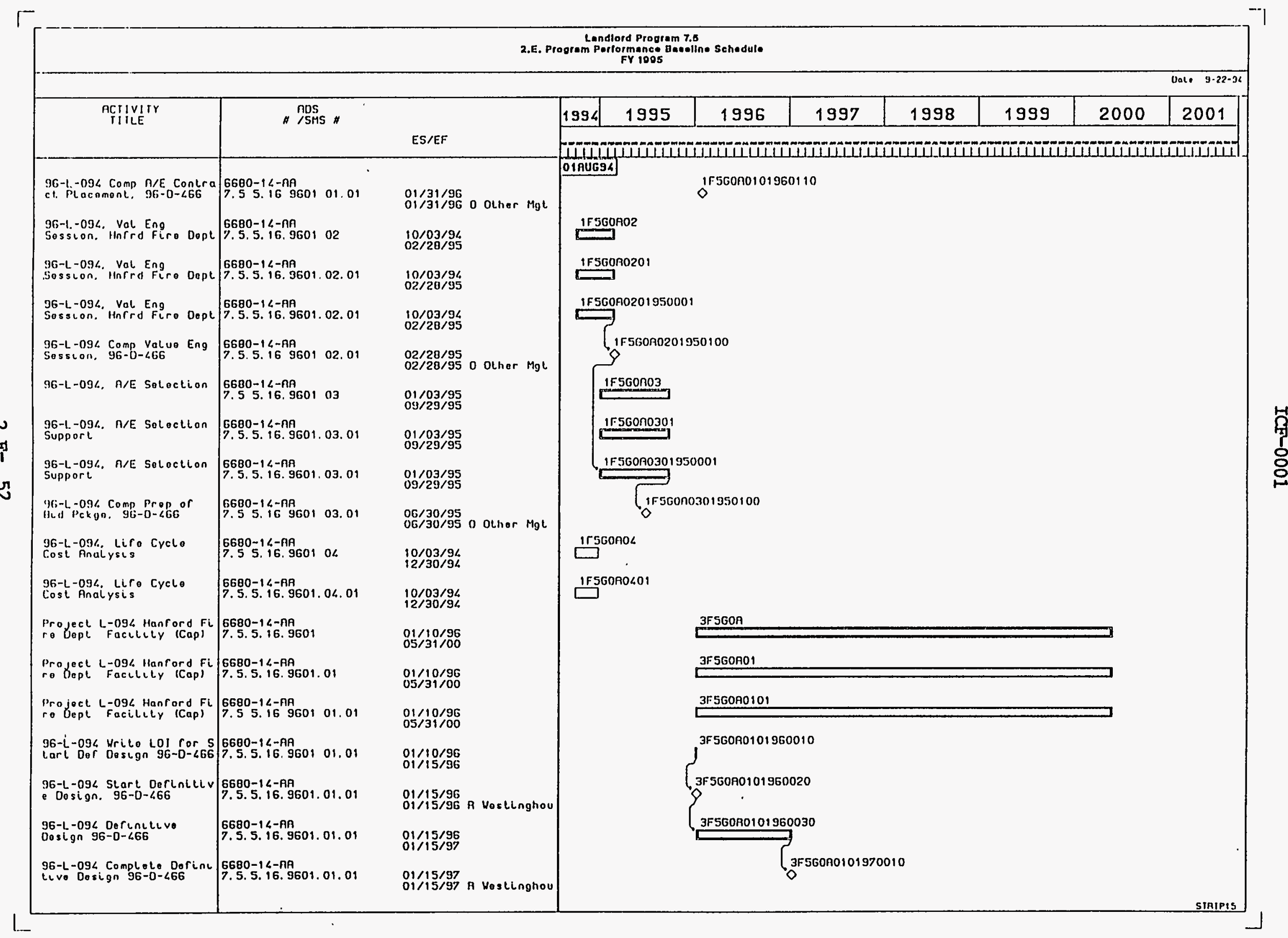




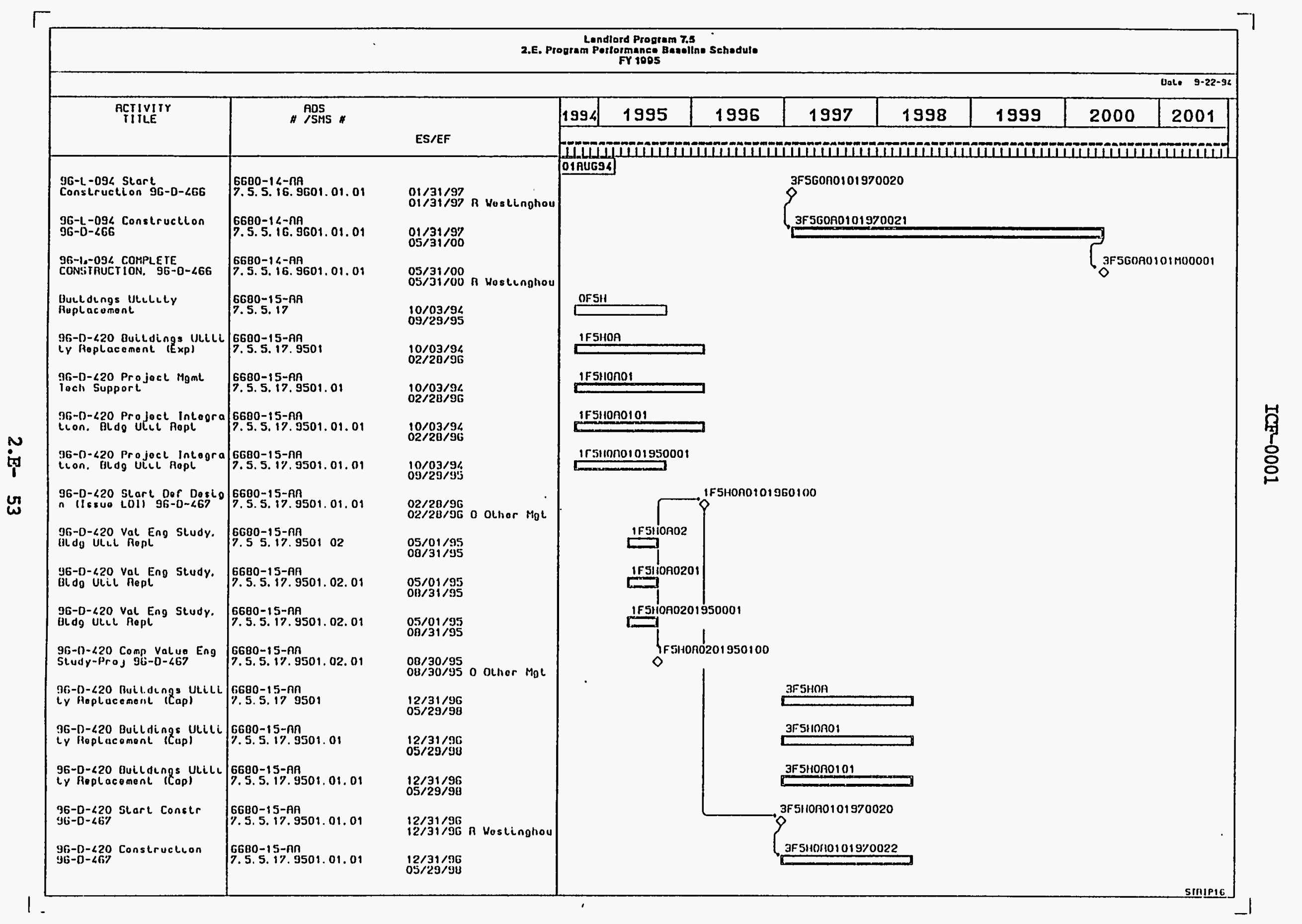




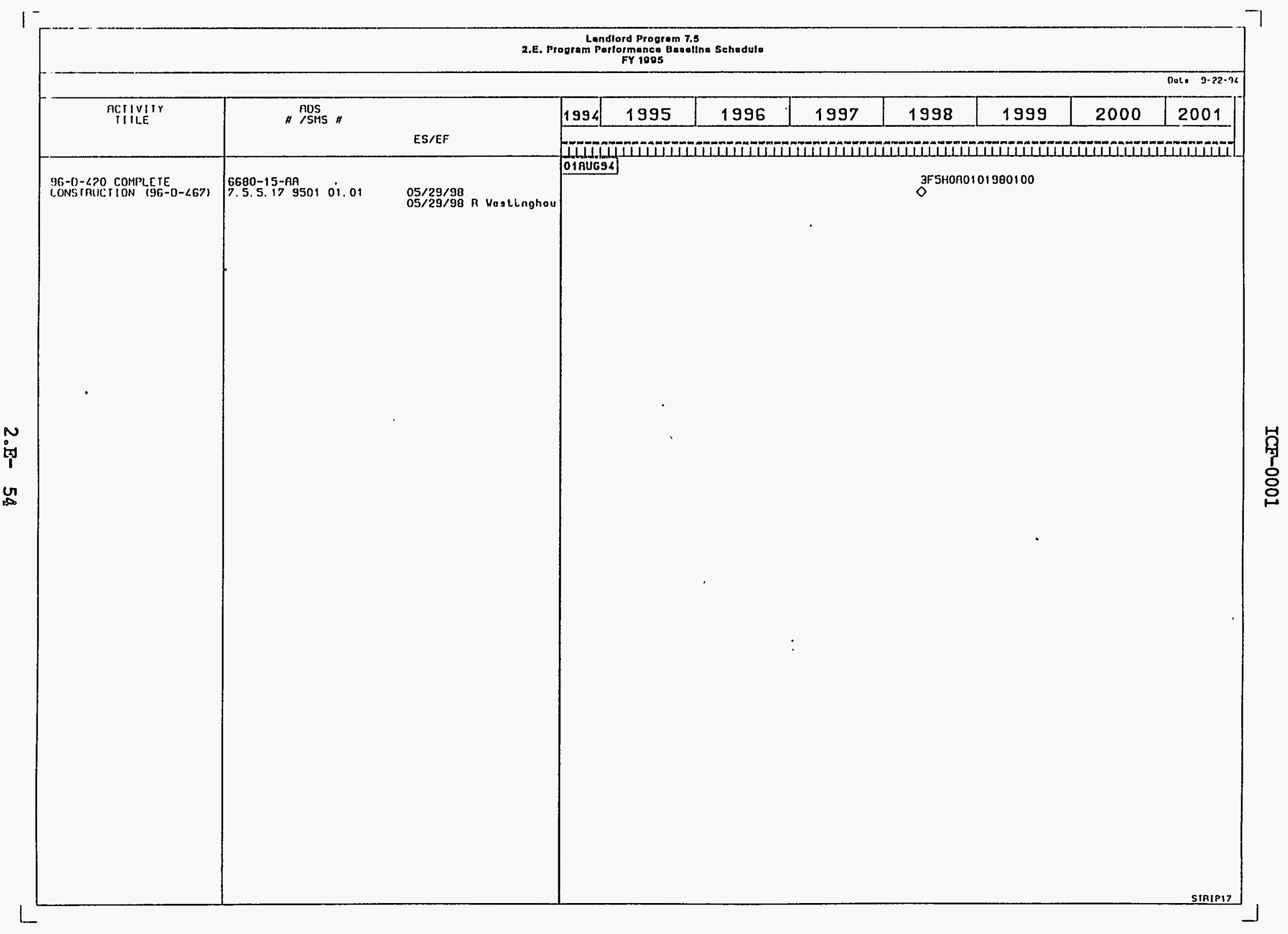




\begin{tabular}{|c|c|c|c|c|c|c|}
\hline \multicolumn{3}{|c|}{ 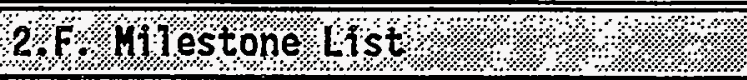 } & \multicolumn{4}{|c|}{ ২, } \\
\hline $\begin{array}{l}\text { Hilestone } \\
\text { (H/S) } \\
\text { Type }\end{array}$ & $\begin{array}{l}\text { M/s Control } \\
\text { Humber }\end{array}$ & WBS Number & $\begin{array}{l}\text { ADS } \\
\text { Number }\end{array}$ & Milestone Description & Due Date & $\begin{array}{l}\text { Comple- } \\
\text { tion } \\
\text { Date }\end{array}$ \\
\hline RL & LLP-95-006 & 7.5 .4 .01 .0001 .04 .01 & $6675-00$ & $\begin{array}{l}\text { Subnit Annual Candidate General } \\
\text { Plant Projects }\end{array}$ & $12 / 01 / 94$ & \\
\hline RL & LLP-95-008 & 7.5 .5 .06 .9401 .01 .01 & $6680-04$ & $\begin{array}{l}94-L-070 \text { Complete Definitive } \\
\text { Design (HQ } 94-0-412 \text { ) }\end{array}$ & $02 / 01 / 95$ & \\
\hline RL & LLP-95-002 & 7.5 .1 .02 .0101 .02 .05 & $6660-00$ & Complete Annual CAMP Report & $02 / 15 / 95$ & \\
\hline $\mathbf{R L}$ & LLP-95-009 & 7.5 .5 .06 .9401 .01 .01 & $6680-04$ & $\begin{array}{l}94-L-070 \text { Start Construction } \\
\text { (HQ } 94-0-412)\end{array}$ & $02 / 28 / 95$ & \\
\hline RL & LLP-95-012 & 7.5 .5 .12 .9201 .01 .01 & $6680-10$ & $\begin{array}{l}\text { 92-L-047 Complete Construction } \\
\text { (HQ 92-D-187) }\end{array}$ & $03 / 17 / 95$ & \\
\hline RL & LLP-95-011 & 7.5 .5 .08 .9201 .01 .01 & $6680-06$ & $\begin{array}{l}92-L-019 \text { Complete Construction } \\
\text { (He } 92-0-185 \text { ) }\end{array}$ & $03 / 28 / 95$ & \\
\hline RL & LLP-95-013 & 7.5 .5 .14 .9301 .01 .01 & $6680-12$ & $\begin{array}{l}93-D-391 \text { Complete Construction } \\
\text { (HQ } 93-D-184)\end{array}$ & $03 / 30 / 95$ & \\
\hline $\begin{array}{l}\text { HQ } \\
\text { TPA }\end{array}$ & $\begin{array}{l}\text { LLP-95-010 } \\
M-17-60 \mathrm{~J}\end{array}$ & 7.5 .5 .06 .9401 .01 .01 & $6680-04$ & $\begin{array}{l}94-L-070 \text { Submit Design } \\
\text { Documentation (HQ } 94-D-412 \text { ) }\end{array}$ & $04 / 28 / 95$ & \\
\hline RL & LLP-95-003 & 7.5 .1 .03 .0101 .02 .01 & $6660-00$ & $\begin{array}{l}\text { Update Hanford Site Development } \\
\text { Plan }\end{array}$ & $04 / 28 / 95$ & \\
\hline RL & LLP-95-007 & 7.5 .5 .01 .0101 .03 .01 & $6680-00$ & $\begin{array}{l}\text { Submit Short Form Data Sheets for } \\
\text { FY } 1999 \text { Candidate Line Items }\end{array}$ & $08 / 01 / 95$ & \\
\hline RL & LLP-95-001 & 7.5 .1 .01 .0101 .02 .01 & $6660-00$ & $\begin{array}{l}\text { Complete Annual Multi-Year Program } \\
\text { Plan }\end{array}$ & $08 / 31 / 95$ & \\
\hline RL & LLP-95-004 & 7.5.2.03.0101.01.00 & $6665-00$ & Complete Demolition Projects & $09 / 29 / 95$ & \\
\hline RL & LLP-95-005 & 7.5.2.05.0101.01.01 & $6665-00$ & $\begin{array}{l}\text { Prepare Renewed Safety Basis for } \\
324 \text { Building }\end{array}$ & $09 / 29 / 95$ & \\
\hline RL & LLP-96-006 & 7.5 .4 .01 .0001 .04 .01 & $66 \sqrt{5}-00$ & $\begin{array}{l}\text { Submit Annual Candidate General } \\
\text { Plant Projects }\end{array}$ & $12 / 01 / 95$ & \\
\hline RL & LLP-96-011 & 7.5 .5 .15 .9601 .01 .01 & $6680-13$ & $\begin{array}{l}96-L-116 \text { Start Definitive Design } \\
\text { (HQ } 96-D-465 \text { ) }\end{array}$ & $01 / 15 / 96$ & \\
\hline RL & LLP-96-007 & 7.5 .4 .02 .9504 .01 .01 & $6675-00$ & $\begin{array}{l}\text { Complete Construction Project } \\
0-446,324 \mathrm{High} \text { Bay Addition }\end{array}$ & $01 / 31 / 96$ & \\
\hline RL & LLP-96-010 & 7.5 .5 .10 .9501 .01 .01 & $6680-08$ & $\begin{array}{l}\text { 95-L-i02 Start Definitive Design } \\
\text { (Issue Letter of Instruction) } \\
\text { (Ha 95-D-453) }\end{array}$ & $01 / 31 / 96$ & \\
\hline $\mathbf{R L}$ & LLP-96-002 & 7.5 .1 .02 .0101 .02 .05 & $6660-00$ & Complete Annual CAMP Report & $02 / 15 / 96$ & \\
\hline RL & LLP-96-003 & 7.5 .1 .03 .0101 .02 .01 & $6660-00$ & $\begin{array}{l}\text { Update Hanford Site Development } \\
\text { Plan }\end{array}$ & $04 / 30 / 96$ & \\
\hline RL & LLP-96-005 & 7.5.2.05.0101.01.01 & $6665-00$ & $\begin{array}{l}\text { Prepare Renewed Safety Basis for } \\
325 \text { Building }\end{array}$ & $04 / 30 / 96$ & \\
\hline
\end{tabular}


FY 1995 MYPP

\section{LANDLORD PROGRAM}

WBS 7.5

\begin{tabular}{|c|c|c|c|c|c|c|}
\hline \multicolumn{7}{|c|}{ 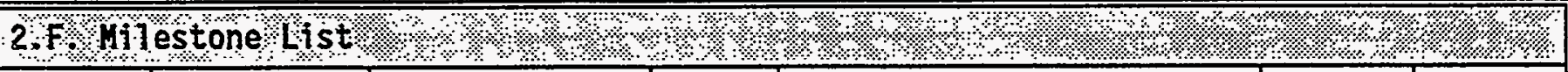 } \\
\hline $\begin{array}{l}\text { Milestone } \\
\text { (M/S) } \\
\text { Type }\end{array}$ & $\begin{array}{l}\text { M/S Control } \\
\text { Number }\end{array}$ & WBS Number & $\begin{array}{l}\text { ADS } \\
\text { Number }\end{array}$ & Milestone Description & Due Date & $\begin{array}{l}\text { Comple- } \\
\text { tion } \\
\text { Date }\end{array}$ \\
\hline RL & LLP-96-008 & 7.5 .5 .01 .0101 .03 .01 & $6680-00$ & $\begin{array}{l}\text { Submit Short Form Data Sheets for } \\
\text { Fy } 2000 \text { Candidate Line I tems }\end{array}$ & $08 / 01 / 96$ & \\
\hline RL & LLP-96-001 & 7.5 .1 .01 .0101 .02 .01 & $6660-00$ & $\begin{array}{l}\text { Complete Annual Multi-Year Program } \\
\text { Plan }\end{array}$ & $08 / 30 / 96$ & \\
\hline RL & $L L P-96-004$ & 7.5 .2 .03 .0101 .01 .00 & $6665-00$ & Complete Demolition Projects & $09 / 30 / 96$ & \\
\hline RL & LLP-96-009 & 7.5 .5 .06 .9401 .01 .01 & $6680-04$ & $\begin{array}{l}\text { 94-L-070 Complete Construction } \\
\text { (HQ } 94-0-412)\end{array}$ & $10 / 01 / 96$ & \\
\hline$R L$ & LLP-97-006 & 7.5 .4 .01 .0001 .04 .01 & $6675-00$ & $\begin{array}{l}\text { Submit Annual Candidate General } \\
\text { Plant Projects }\end{array}$ & $12 / 02 / 96$ & \\
\hline RL & LLP-97-005 & 7.5 .2 .05 .0101 .01 .01 & $6665-00$ & $\begin{array}{l}\text { Prepare Renewed Safety Basis for } \\
327 \text { Building }\end{array}$ & $12 / 31 / 96$ & \\
\hline RL & LLP-97-009 & 7.5 .5 .10 .9501 .01 .01 & $6680-08$ & $\begin{array}{l}95-L-102 \text { Start Construction } \\
\text { (Ha } 95-D-453 \text { ) }\end{array}$ & $01 / 15 / 97$ & \\
\hline RL & LLP-97-002 & 7.5 .1 .02 .0101 .02 .05 & $6660-00$ & Complete Annual CAMP Report & $02 / 28 / 97$ & \\
\hline RL & LLP-97-003 & 7.5 .1 .03 .0101 .02 .01 & $6660-00$ & $\begin{array}{l}\text { Update Hanford Site Development } \\
\text { Plan }\end{array}$ & $04 / 30 / 97^{\circ}$ & \\
\hline $\mathbf{R L}$ & LLP-97-010 & 7.5 .5 .15 .9601 .01 .01 & $6680-13$ & $\begin{array}{l}96-L-116 \text { Start Construction } \\
\text { (HQ } 96-0-465)\end{array}$ & $06 / 13 / 97$ & \\
\hline $\begin{array}{l}\text { HQ } \\
\text { TPA }\end{array}$ & $\begin{array}{l}\text { LLP }-97-008 \\
H-17-06 K\end{array}$ & 7.5 .5 .06 .9401 .09 .01 & $6680-04$ & $\begin{array}{l}\text { 94-L-070 Replace Process Sewer } \\
\text { Piping (HO } 94-D-412 \text { ) }\end{array}$ & $06 / 30 / 97$ & \\
\hline $\mathbf{R L}$ & LLP $-97-007$ & 7.5 .5 .01 .0101 .03 .01 & $6680-00$ & $\begin{array}{l}\text { Submit Short Form Data Sheets for } \\
\text { FY } 2001 \text { Candidate Line Items }\end{array}$ & $08 / 01 / 97$ & \\
\hline $\mathbf{R L}$ & LLP-97-001 & 7.5 .9 .01 .0101 .02 .01 & $6660-00$ & $\begin{array}{l}\text { Complete Annual Multi-Year Program } \\
\text { Plan }\end{array}$ & $08 / 29 / 97$ & \\
\hline RL & LLP-97-004 & 7.5 .2 .03 .0101 .01 .00 & $6665-00$ & Complete Demolition Projects & $09 / 30 / 97$ & \\
\hline
\end{tabular}

Note: Interim milestones in support of HQ and RL type milestones can be found in Section 2.E, "Program Performance Basel ine Schedule." 
$2 G$.

ICF Kaiser Hanford Company MILESTONE DESCRIPTION SHEET

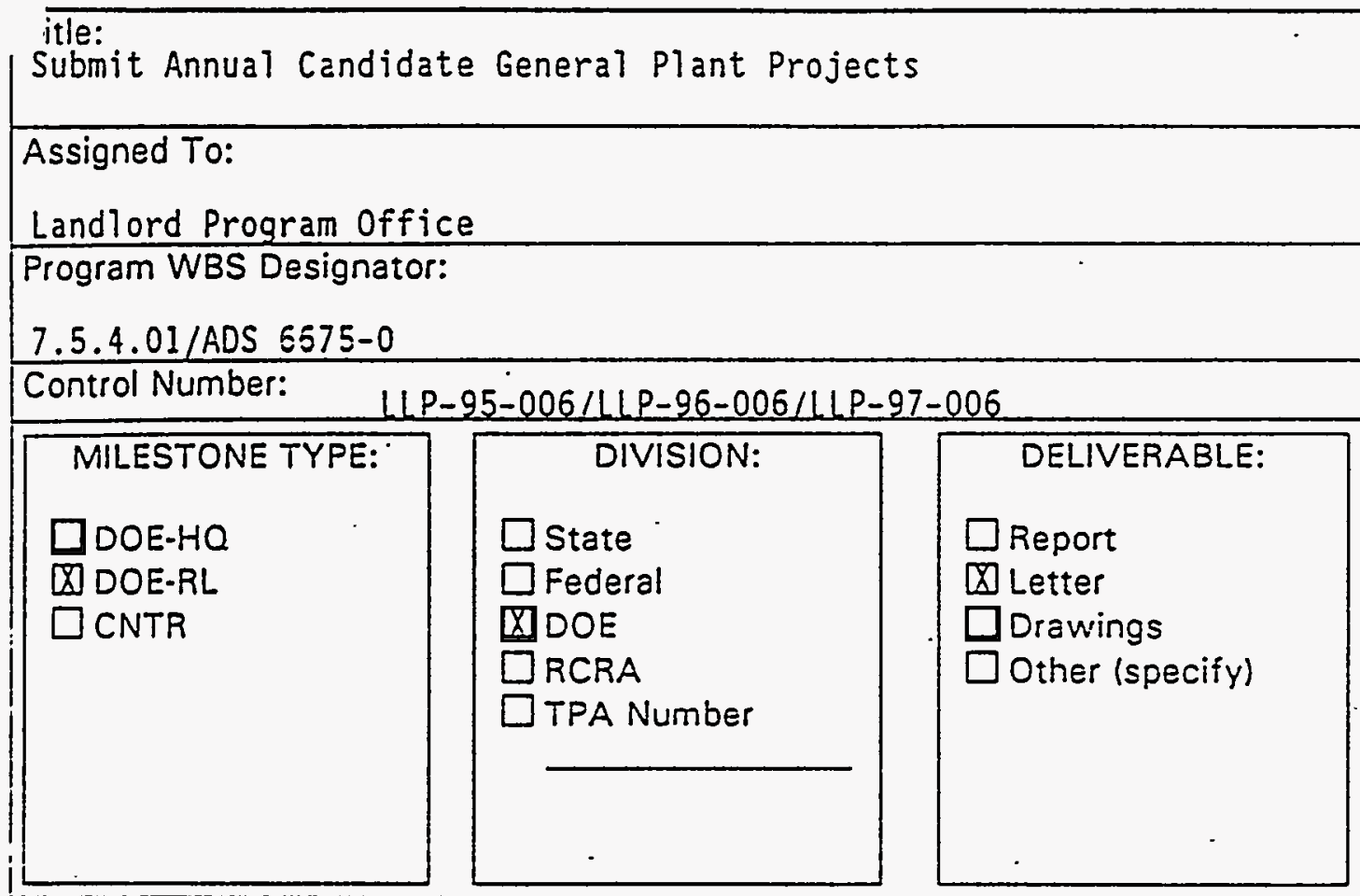

Date: $08 / 31 / 94$

CIN:

Due Date: Dec. Rev.: 0

Milestone description:

Submit Annual Candidate Project Data Sheets (CPDS) to DOE-RL for General Plant Projects (GPP).

Start Date: October Annually Description of what constitutes completion of this milestone:

Prepare CPDS for each GPP and transmit to DOE-RL by formal letter at the begining of December annually.

Cost Account Nianager

O. Q. Oanch A. Danch Program Elemenit. fianager

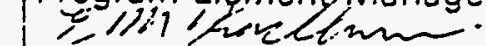
E. M. Koellermeier

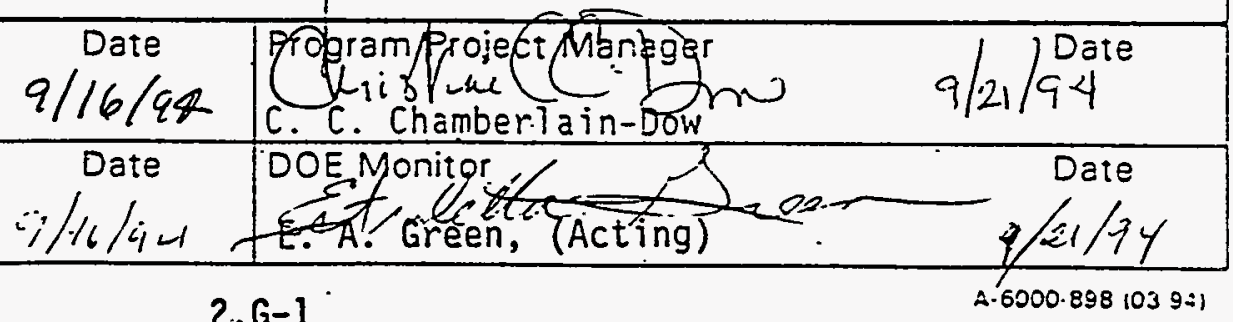


$2 G$.

ICF Kaiser Hanford Company MILESTONE DESCRIPTION SHEET

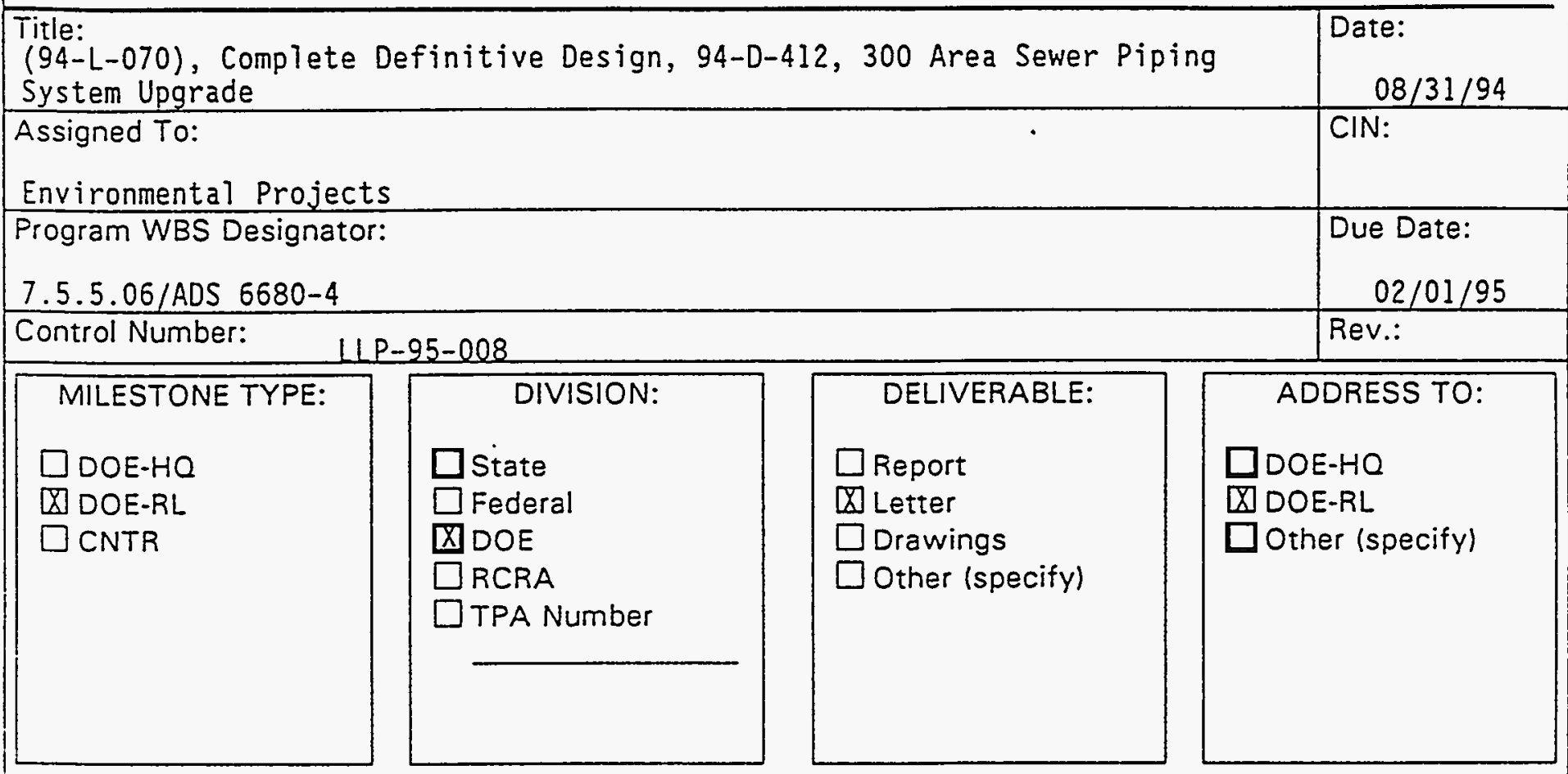

Milestone description:

Complete definitive design.

Description of what constitutes completion of this milestone:

WHC approval of all drawings and specifications.

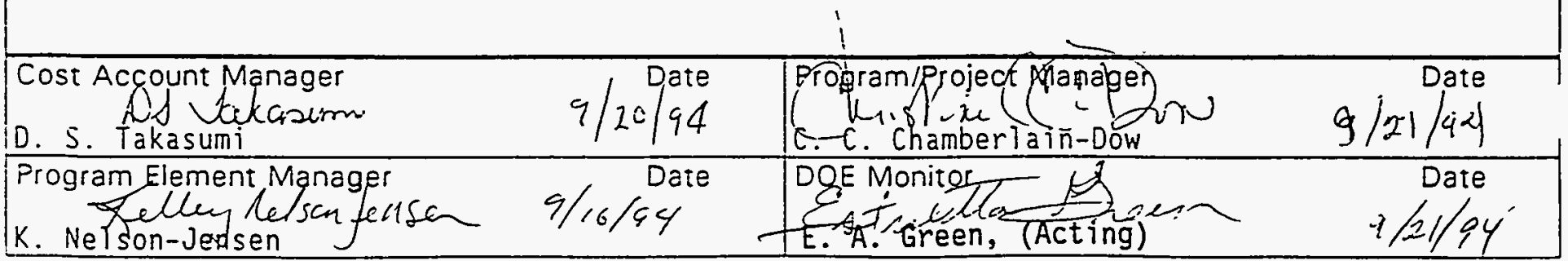


$2 G$.

ICF Kaiser Hanford Company MILESTONE DESCRIPTION SHEET

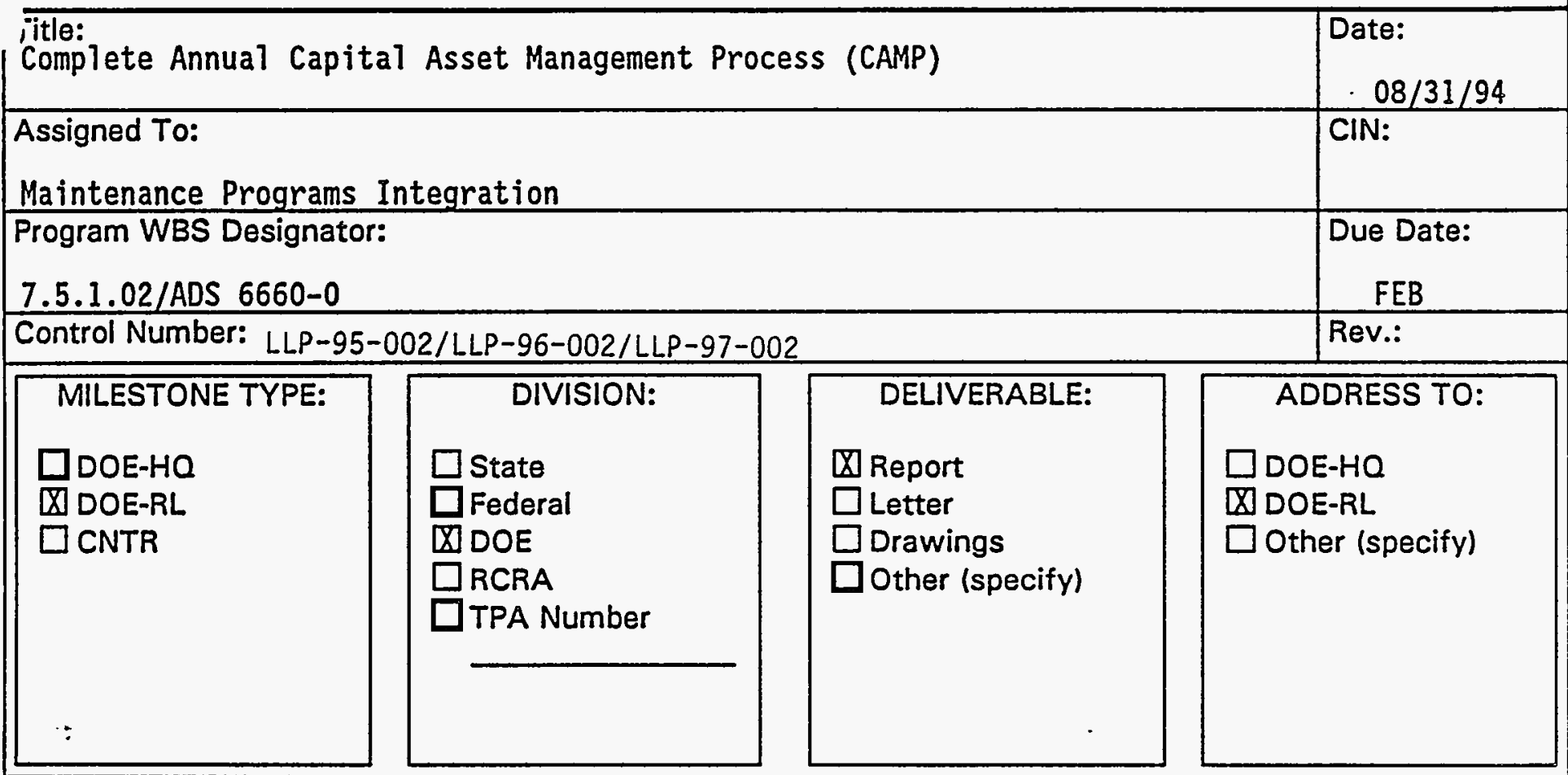

Milestone description:

CAMP defines a credible and defendable process to identify the funding required to maintain a capital asset in working condition over the life of the asset. The funding requirements are produced in a planning process that takes into account the current condition of the asset, its intended function and what. actions are required to maintain the asset. It produces a $\mathrm{planning}$ document, the CAMP report, to support, justify, and prioritize budget and project requests. The CAMP report is a snapshot of the ongoing cycle.

Start Date:

October, Annually

Description of what constitutes completion of this milestone:

CAMP report submitted annually to DOE-RL in February.

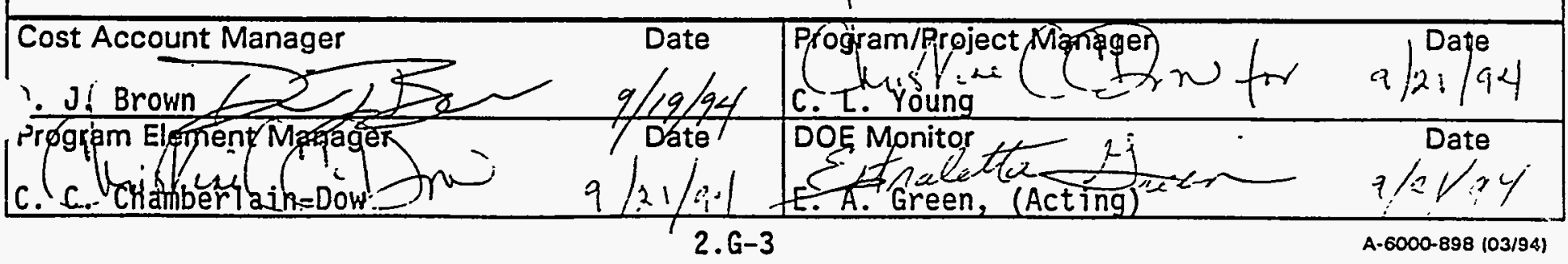


Title:

(94-L-070), Start Construction, 94-D-412, 300 Area Process Sewer Piping System Upgrade

Assigned To:

Environmental Projects

Program WBS Designator:

7.5.5.06/ADS 6E80-4

Control Number:

\begin{tabular}{|c|c|}
\hline $\begin{array}{l}\text { MILESTONE TYPE: } \\
\square \text { DOE-HQ } \\
\text { 囚 DOE-RL } \\
\square \text { CNTR }\end{array}$ & $\begin{array}{l}\text { DIVISION: } \\
\square \text { State } \\
\square \text { Federal } \\
\text { XDOE } \\
\square \text { RCRA } \\
\square \text { TPA Number }\end{array}$ \\
\hline & \\
\hline
\end{tabular}

Nilestone description:

Start Construction.
Description of what constitutes completion of this milestone:

HHC issue a letter of instruction to ICF KH to initiate construction procurement activities.

\begin{tabular}{|c|c|c|c|}
\hline $\begin{array}{l}\text { Cost Account Menager } \\
\text { J. S. Takasumi Ad dakedemi }\end{array}$ & $\begin{array}{l}\text { Date } \\
9 / \mathrm{s} / \mathrm{s} ; 4\end{array}$ & $\begin{array}{l}\text { Progripm/Project Mapre } \\
\text { c. E. Chamber ain-t }\end{array}$ & $9 / 21 / 94$ \\
\hline $\begin{array}{l}\text { Frogram Element Nienager } \\
\because \text { Hel son-Jense felleythetson fens }\end{array}$ & $\begin{array}{l}\text { Date } \\
9 / 19 / 94\end{array}$ & 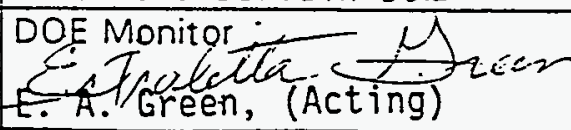 & Date \\
\hline
\end{tabular}


$2 G$;

\section{ICF Kaiser Hanford Company \\ MILESTONE DESCRIPTION SHEET}

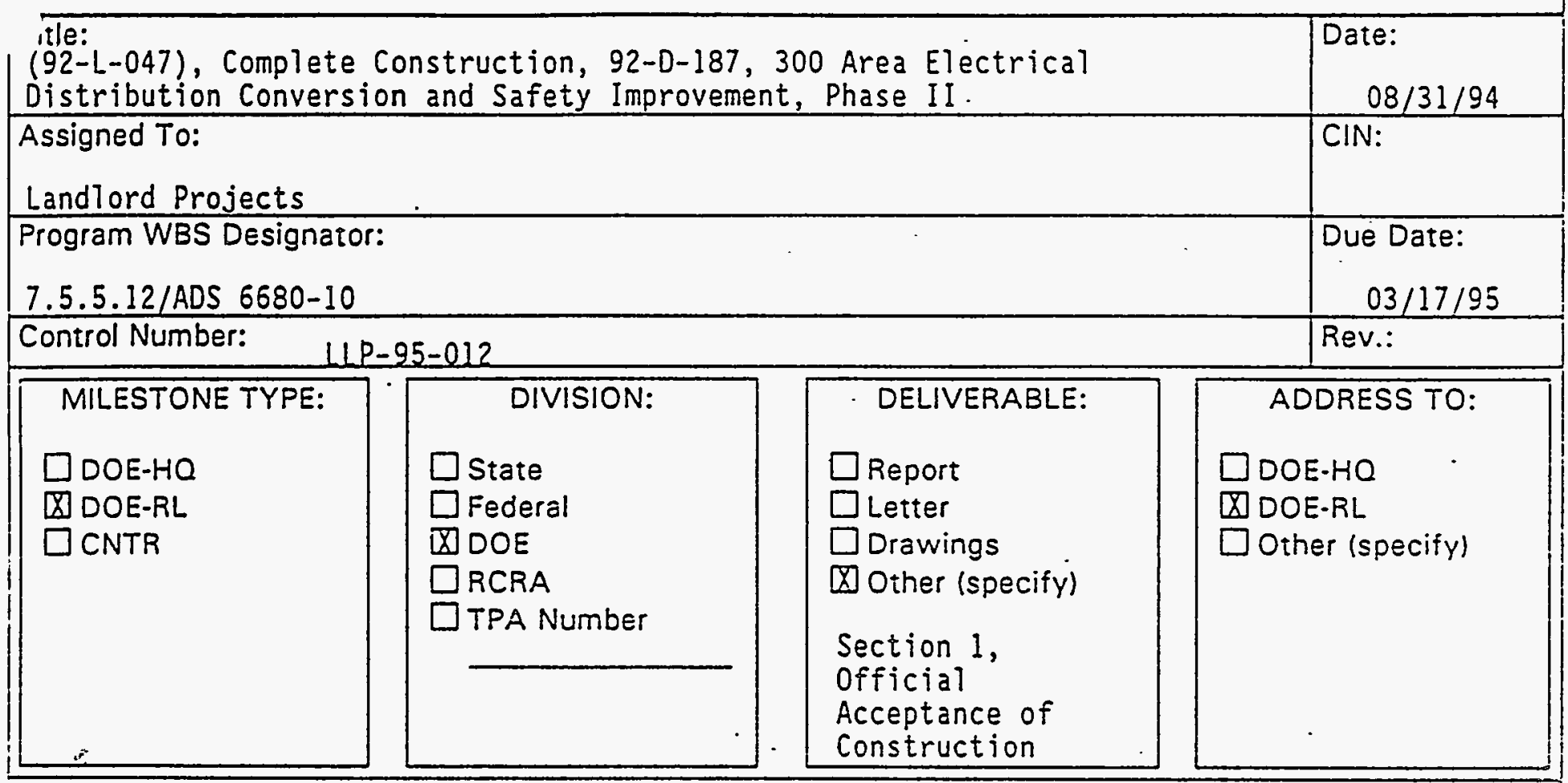

Milestone description:

Complete construction.

Description of what constitutes completion of this milestone:

DOE-RL and field engineering approval of Official Acceptance of

Construction, Section 1 .

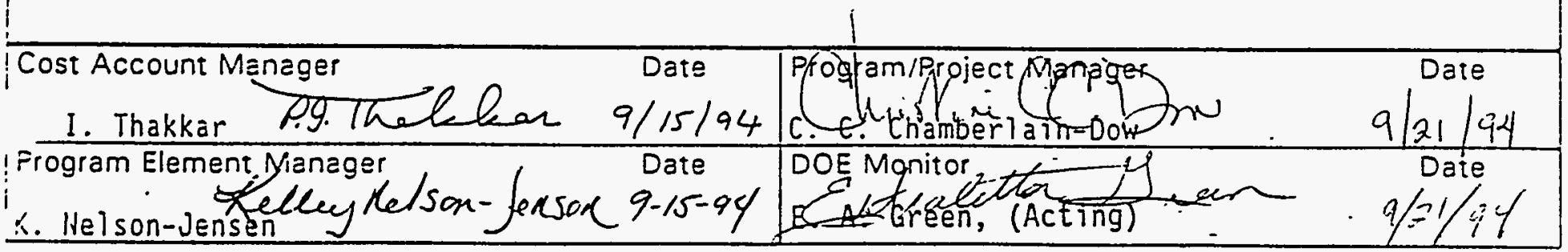


$2 G$.

ICF Kaiser Hanford Company

MILESTÓNE DESCRIPTIÖN SHEETT

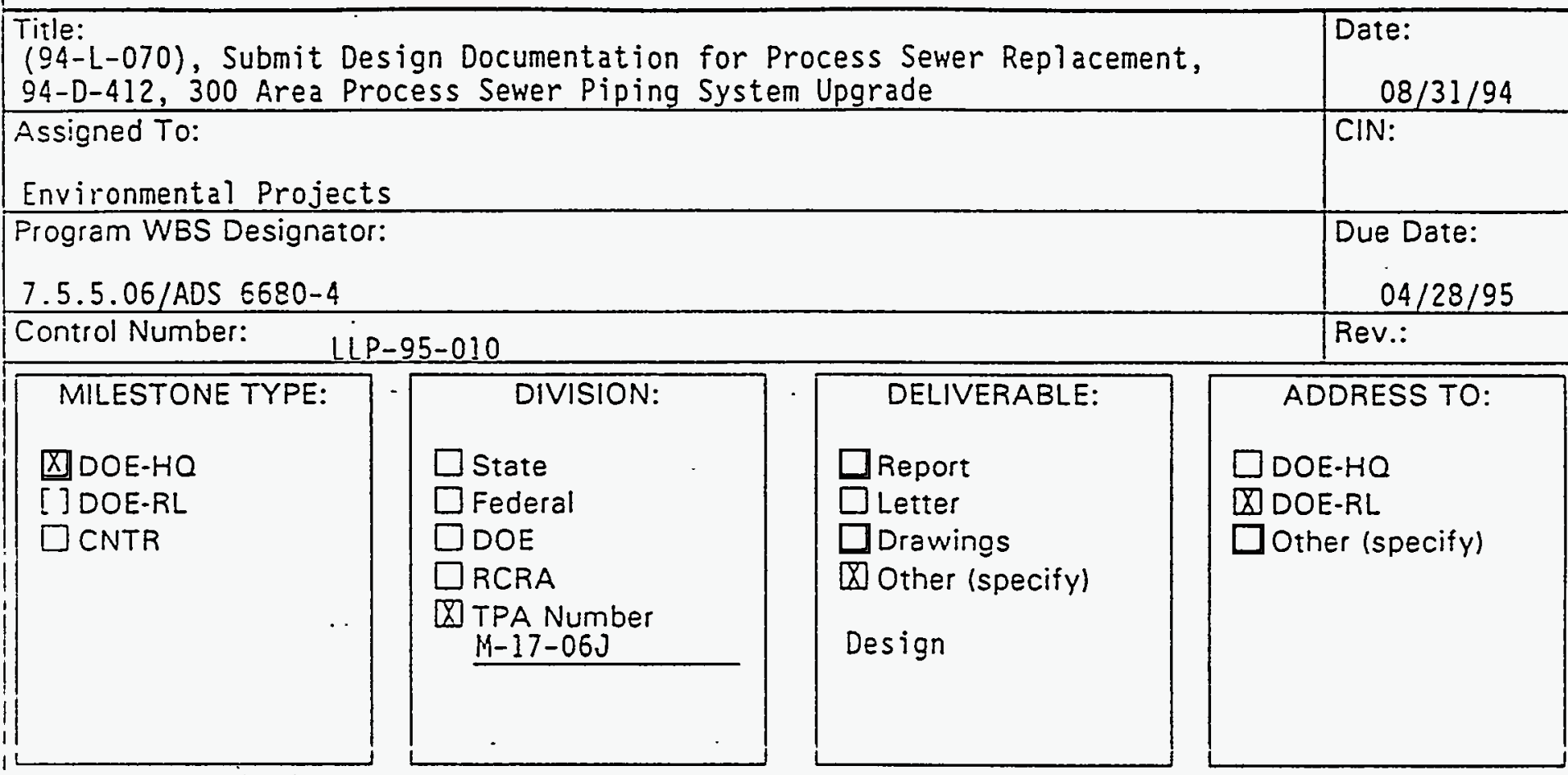

Milestone description:

Submit design documentation to Washington Department of Ecology (WDOE) and to the Environmental Protection Agency (EPA).

Description of what constitutes completion of this milestone:

WHC to transmit design documentation to RL for their submittal to WDOE and EPA.

\begin{tabular}{|c|c|c|c|}
\hline $\begin{array}{l}\text { Mienager } \\
\text { umi } \hat{L}, y \text { Uakcosum }\end{array}$ & $\begin{array}{l}\text { Date } \\
-\$ 9 / 94\end{array}$ & Mil-Dort & $9 / 21 / 94$ \\
\hline $\begin{array}{c}\text { ogram Element Vianager } \\
\text { *elleydelsen fen } \\
\text { Hel son-Jens?n }\end{array}$ & $\begin{array}{l}\text { Date } \\
9 / 19 / 44 \mid\end{array}$ & $\begin{array}{l}\text { DOE Monitor th H } \\
\text { E. At Geen, (Acting) }\end{array}$ & $9 / 21 / 94$ \\
\hline
\end{tabular}


$2 G$.

ICF Kaiser Hanford Company

MILESTÓNE DESCRIPTION SHEET

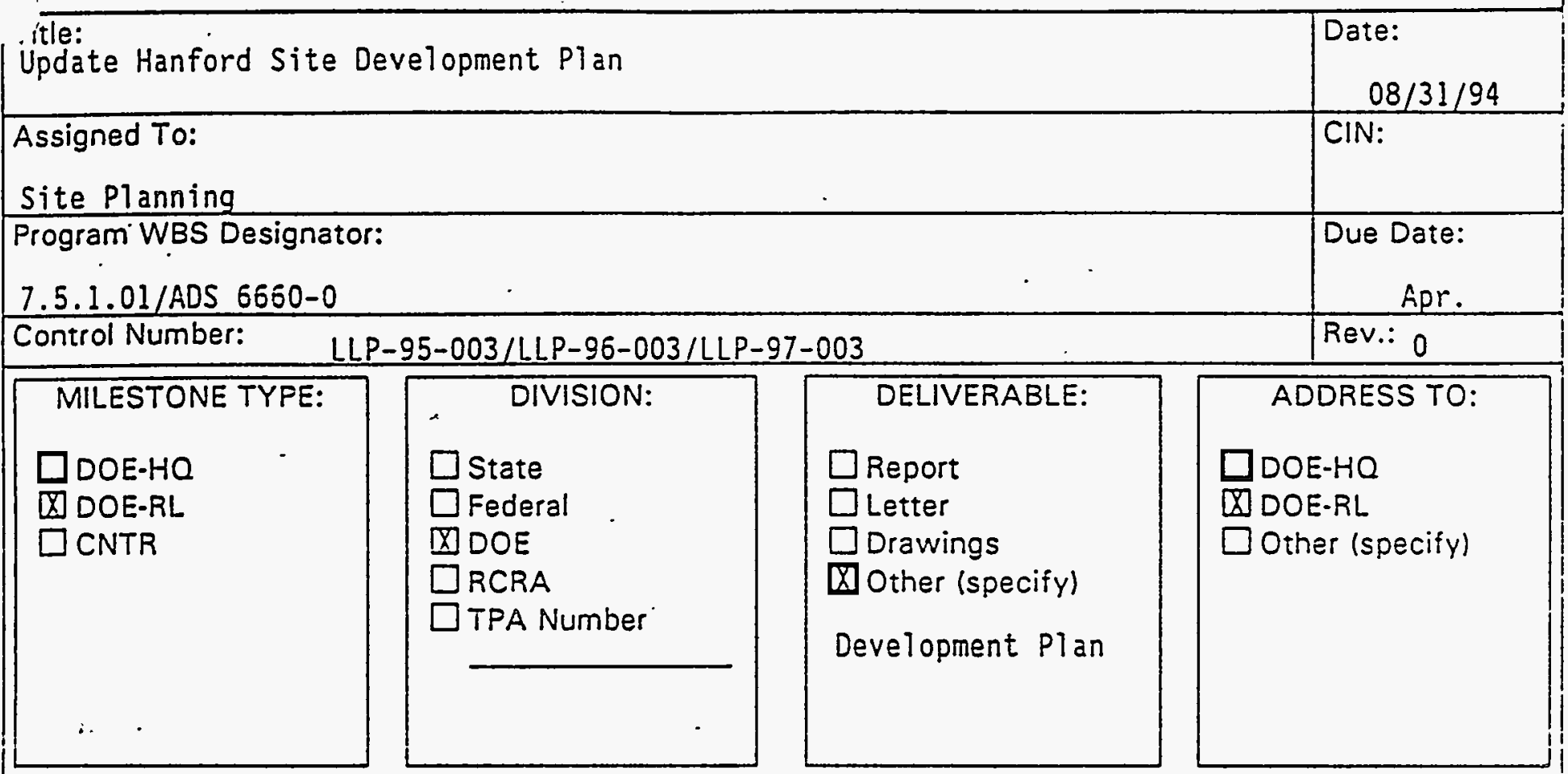

Milestone description:

The Development Plan for the Hanford Site is reviewed and revised annually based on current information.

Start date: February Annually

Description of what constitutes completion of this milestone:

Deliver updated final draft to DOE-RL at the end of April, annually.

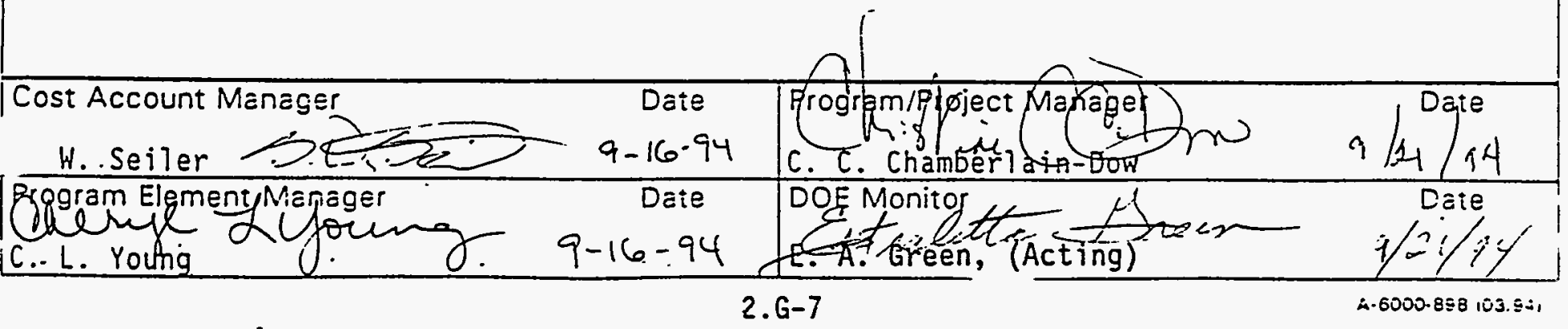


Tiile:

(92-L-019), Complete Construction, 92-D-185, Road, Ground, and Lighting Safety Improvements, $300 / 1100$ Areas

Assigned To:

Lendlord Projects

Program WBS Designator:

7.5.5.08/ADS 6680-6

Due Date:

Control Number:

UP $-25-0] 1$

$03 / 28 / 95$

\begin{tabular}{|c|c|c|c|}
\hline $\begin{array}{l}\text { MILESTONE TYPE: } \\
\square \text { DOE-HO } \\
\text { వ DOE-RL } \\
\square \text { CNTR }\end{array}$ & $\begin{array}{l}\text { DIVISION: } \\
\square \text { State } \\
\square \text { Federal } \\
\square \text { DOE } \\
\square \text { RCRA } \\
\square \text { TPA Number }\end{array}$ & $\begin{array}{l}\text { DELIVERABLE: } \\
\square \text { Report } \\
\square \text { Letter } \\
\square \text { Drawings } \\
\text { O Other (specify) } \\
\text { Section 1, } \\
\text { Official } \\
\text { Acceptance of } \\
\text { Construction }\end{array}$ & $\begin{array}{l}\text { ADDRESS TO: } \\
\square \text { DOE-HO. } \\
\square \text { DOE-RL } \\
\square \text { Other (specify) } \\
\text { ICF KH }\end{array}$ \\
\hline
\end{tabular}

Milestone descripition:

Complete construction.

Description of what constitutes completion of this milestone:

DOE-RL and ICF KH field engineering approval of Official Acceptance of

Construction, Section 1.

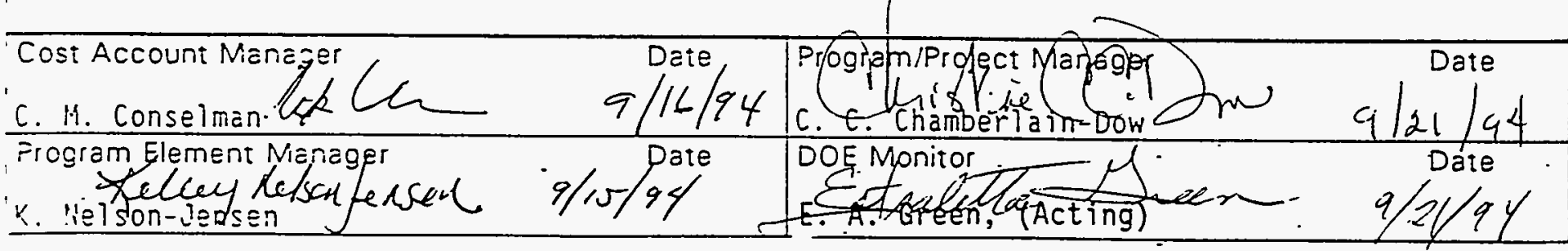


$2 G$

ICF Kaiser Hanford Company MILESTONE DESCRIPTION SHEET

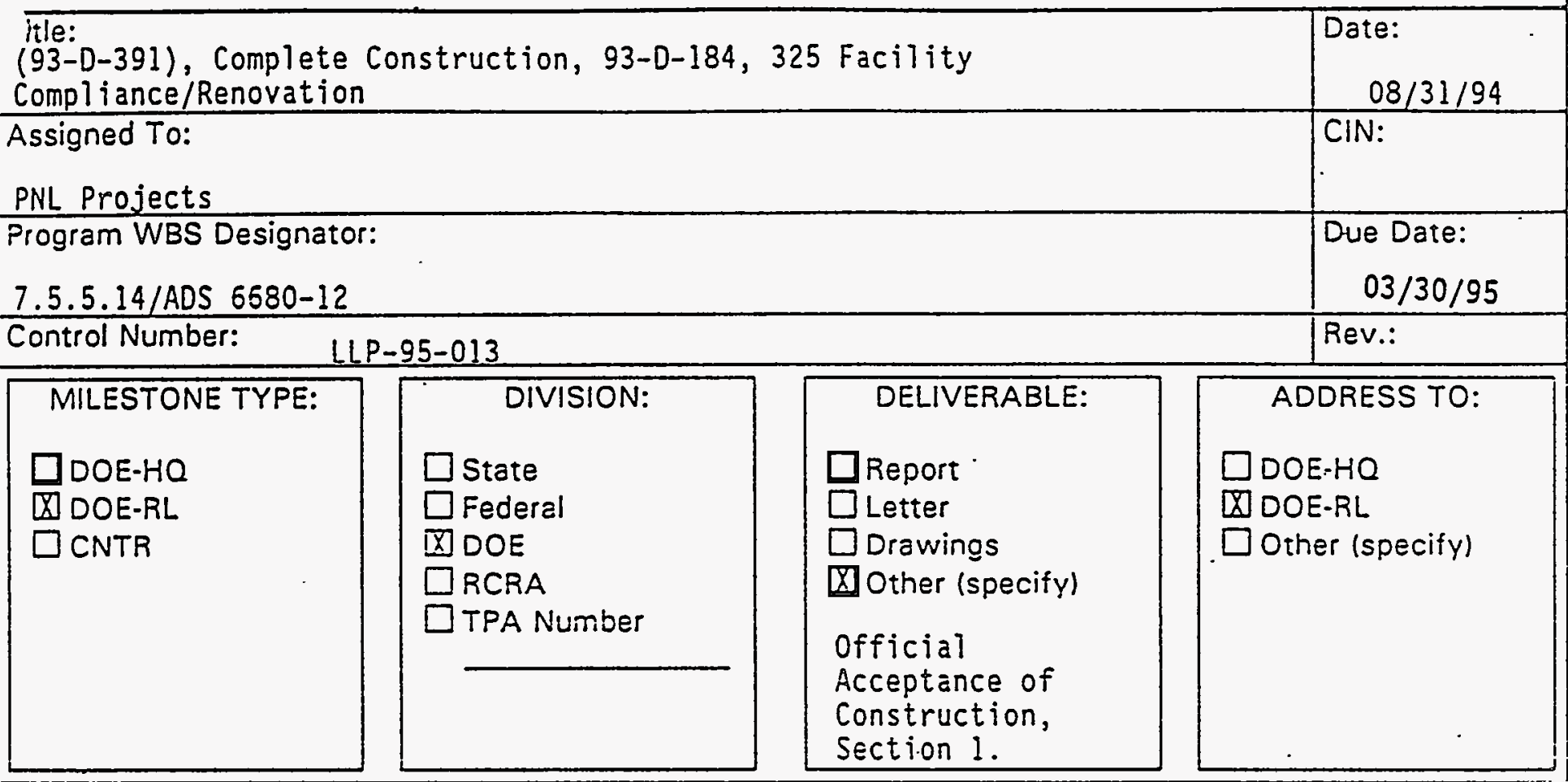

Milestone description:

Complete construction for Project D-391, 325 Facility Compliance/Renovation.

Description of what constitutes completion of this milestone:

DOE-RL and PNL approval of Official Acceptance of Construction, Section 1.

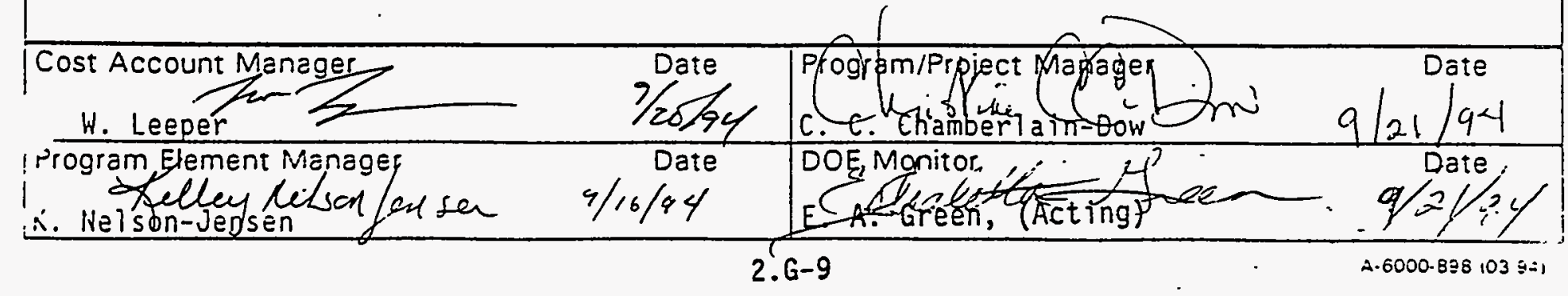




\title{
ICF Kaiser Hanford Company MILESTONE DESCRIPTION SHEET
}

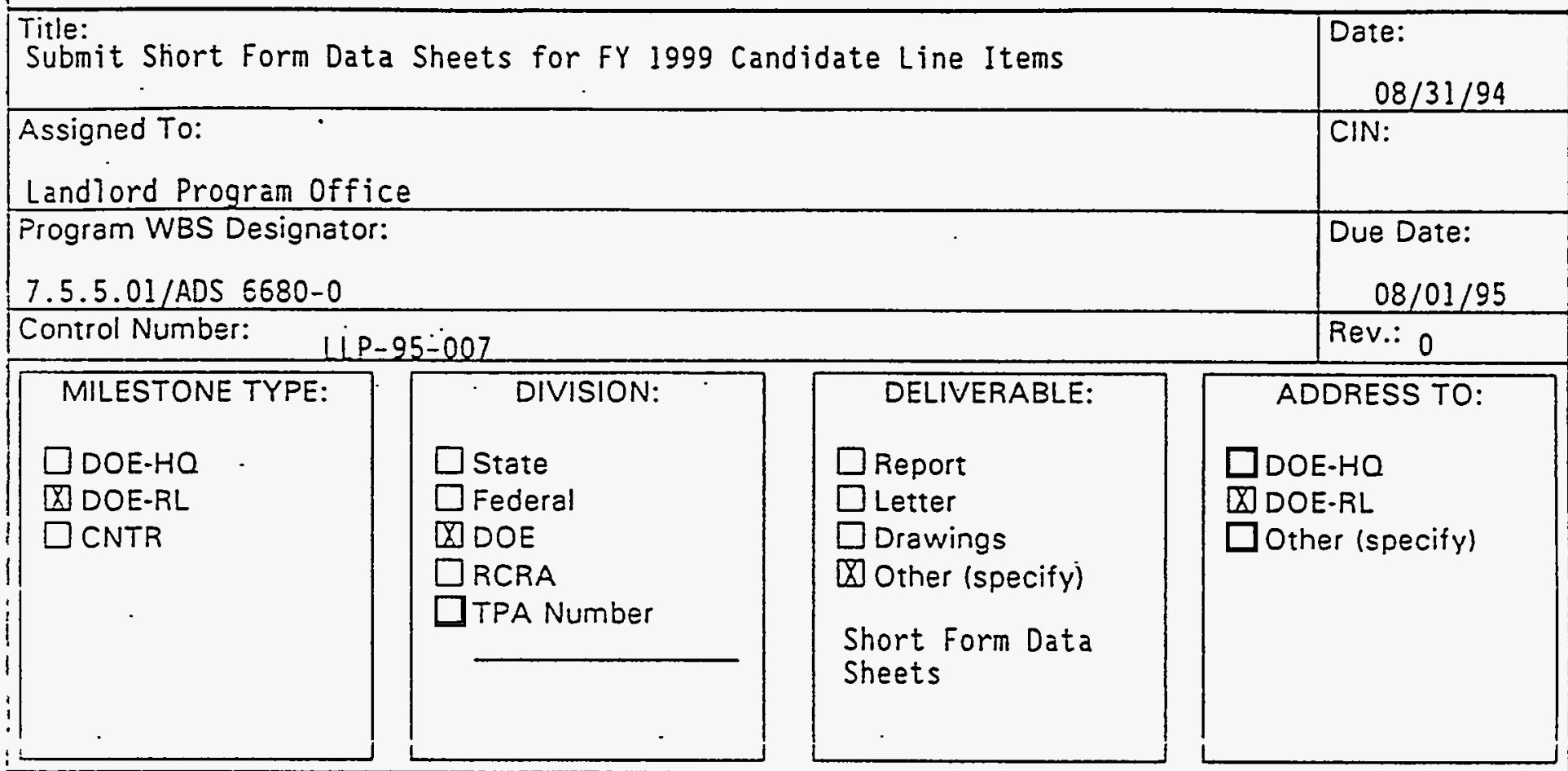

\footnotetext{
Milestone description:

Transmit Short Form Data Sheets for FY 1999 Candidate Line Items to DOE-RL.
}

\begin{abstract}
Description of what constitutes completion of this milestone:
Issue transmittal letter to DOE-RL with completed Short Form Data Sheets for FY 1999 Candidate Line Item projects.
\end{abstract}

Cost Account Manager D. A. Danch Daie
Danchel 9 . Oauch
Danch i Program Element Manager

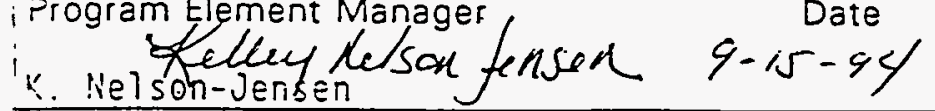


$2 G$.

ICF Kaiser Hanford Company

MILESTONE DESCRIPTION SHEET

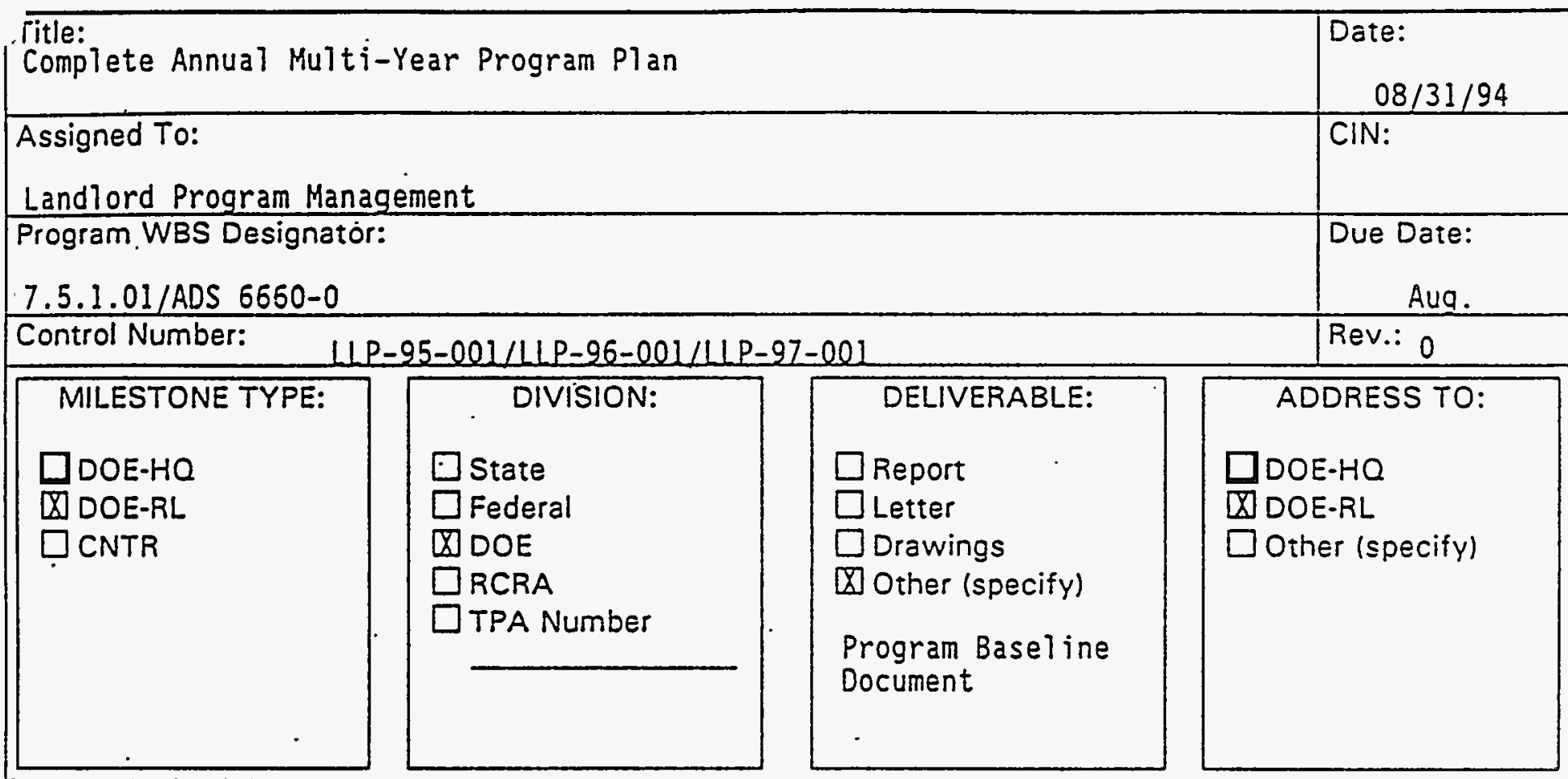

Milestone description:

The purpose of the Muiti-Year Program Plan is to establish the program's

technical, schedule, and cost baseline for the life of the program. Logic

diagrams, basis and assumptions, and scope statements are also developed.

The docunient also includes current year execution information.

Start Date: May Annually

Description of what constitutes completion of this milestone:

Submit document to DOE-RL by the required due date. Obtain DOE-RL approval prior to the beginning of the fiscal year. This milestone will be completed annually at the end of August.

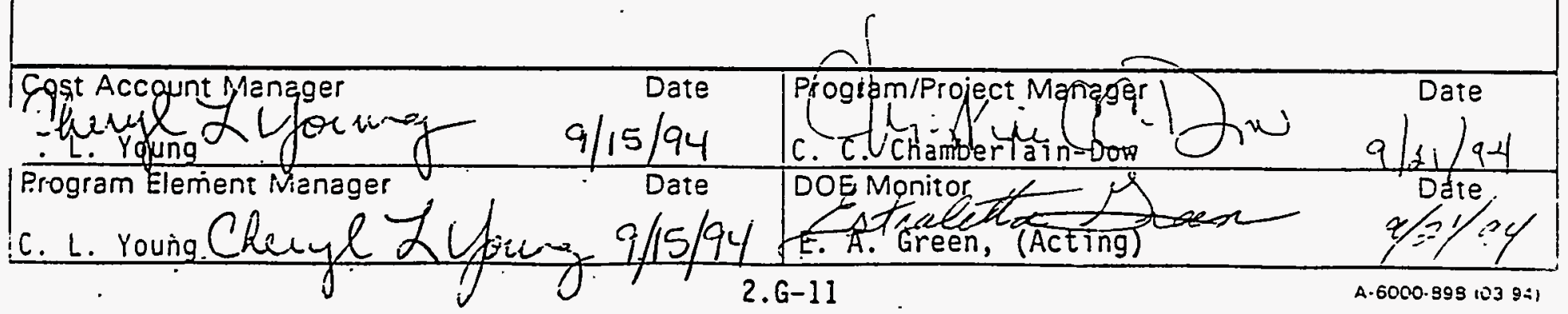


$2 G$.

ICF Kaiser Hanford Company MILESTONE DESCRIPTION SHEET

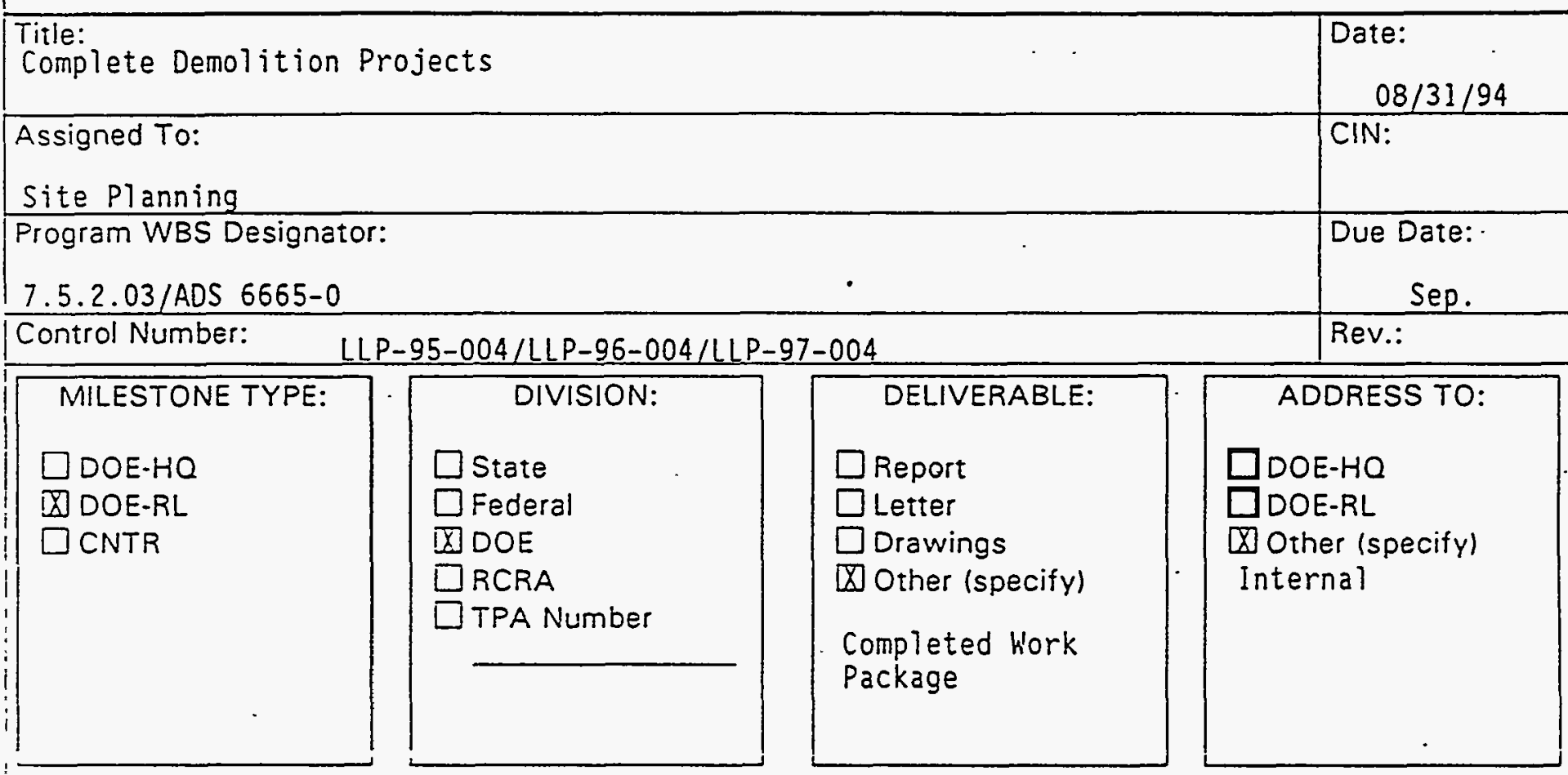

Milestone description:

This milestone signifies annual completion of all demolition projects per the Multi-Year Program Plan (MYPP). The MYPP defines the structures and schedules for demolition by this WBS.

Description of what constitutes completion of this milestone:

The milestone is considered complete when all JCS packages for demolitions are signed-off as complete. The milestone will be compléted annually by the end of September.

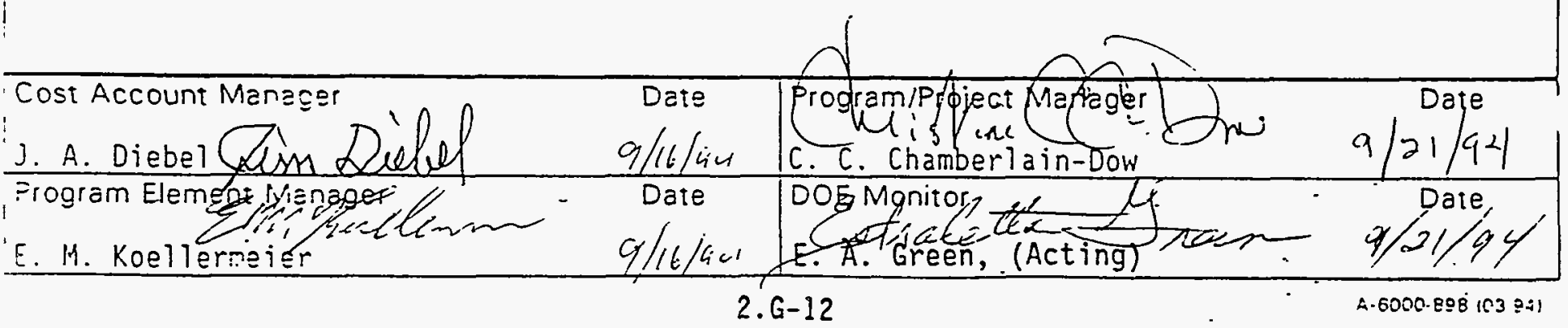


$2 G$.

ICF Kaiser Hanford Company MILESTONE DESCRIPTION SHEET

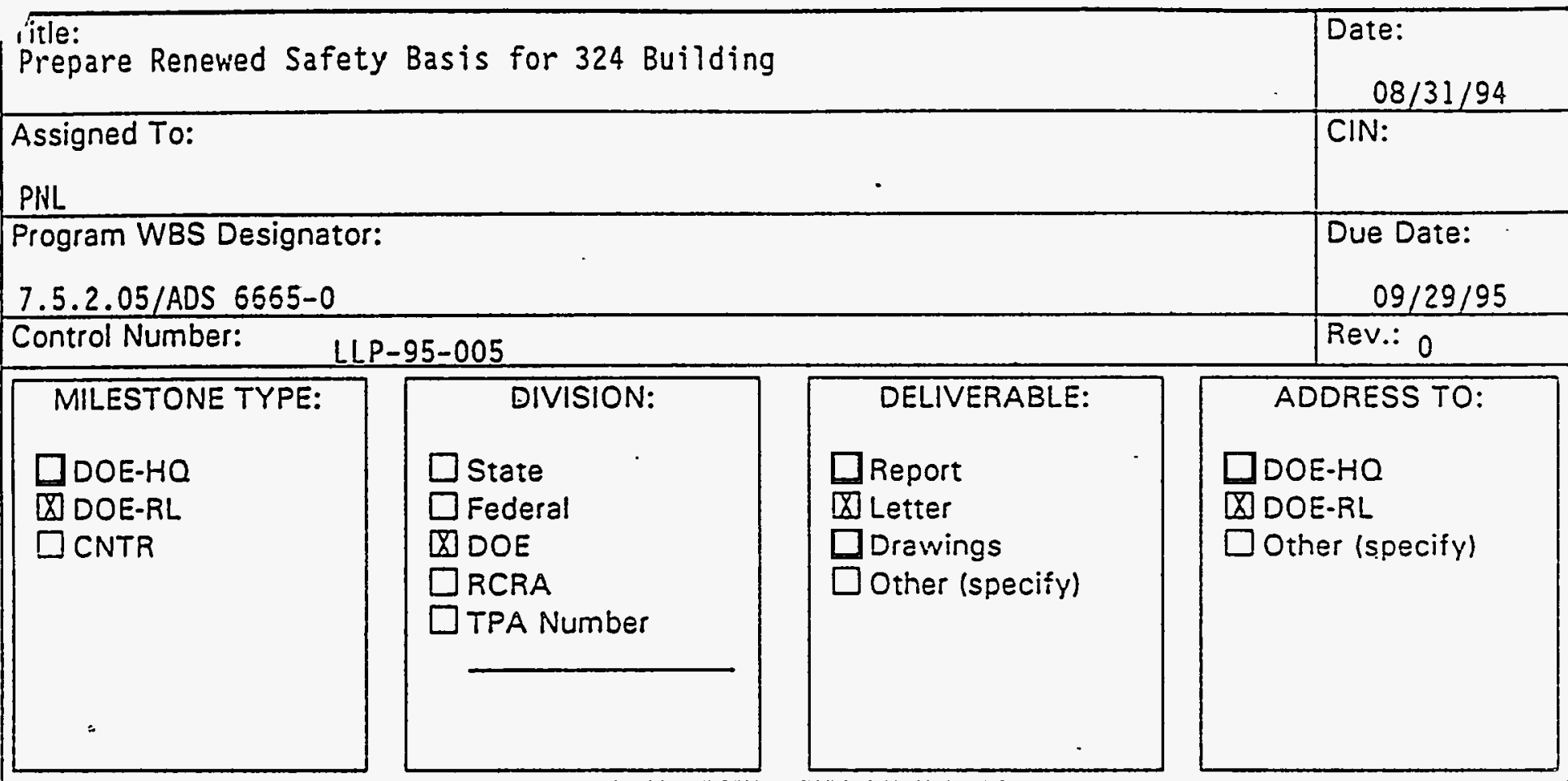

Milestone description:

Prepare renewed safety basis for 324 Building in support of preparing updated facility Technical Safety Requirements and Safety Analysis Reports.

Start Date: $09 / 30 / 94$

Description of what constitutes completion of this milestone:

Prepare renewed safety basis in accordance with PNL's "Transition Plan for Achieving Compliance with DOE 5480.22 and DOE 5480.23," Revision 1, August 1993. Obtain PNL independent technical reviews and resolve comments. Obtain approval from PNL's Safety Review Council. Submit document to DOE-RL for approval.

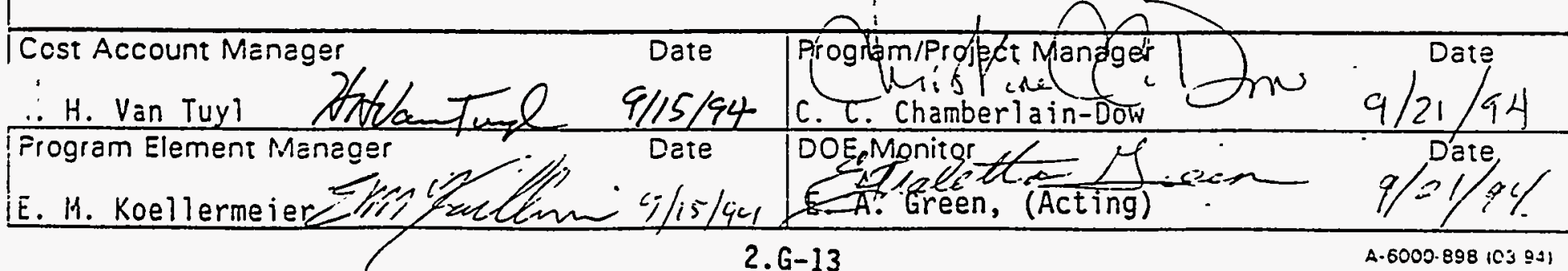


$2 G$.

ICF Kaiser Hanford Company

MILESTONE DESCRIPTION SHEET

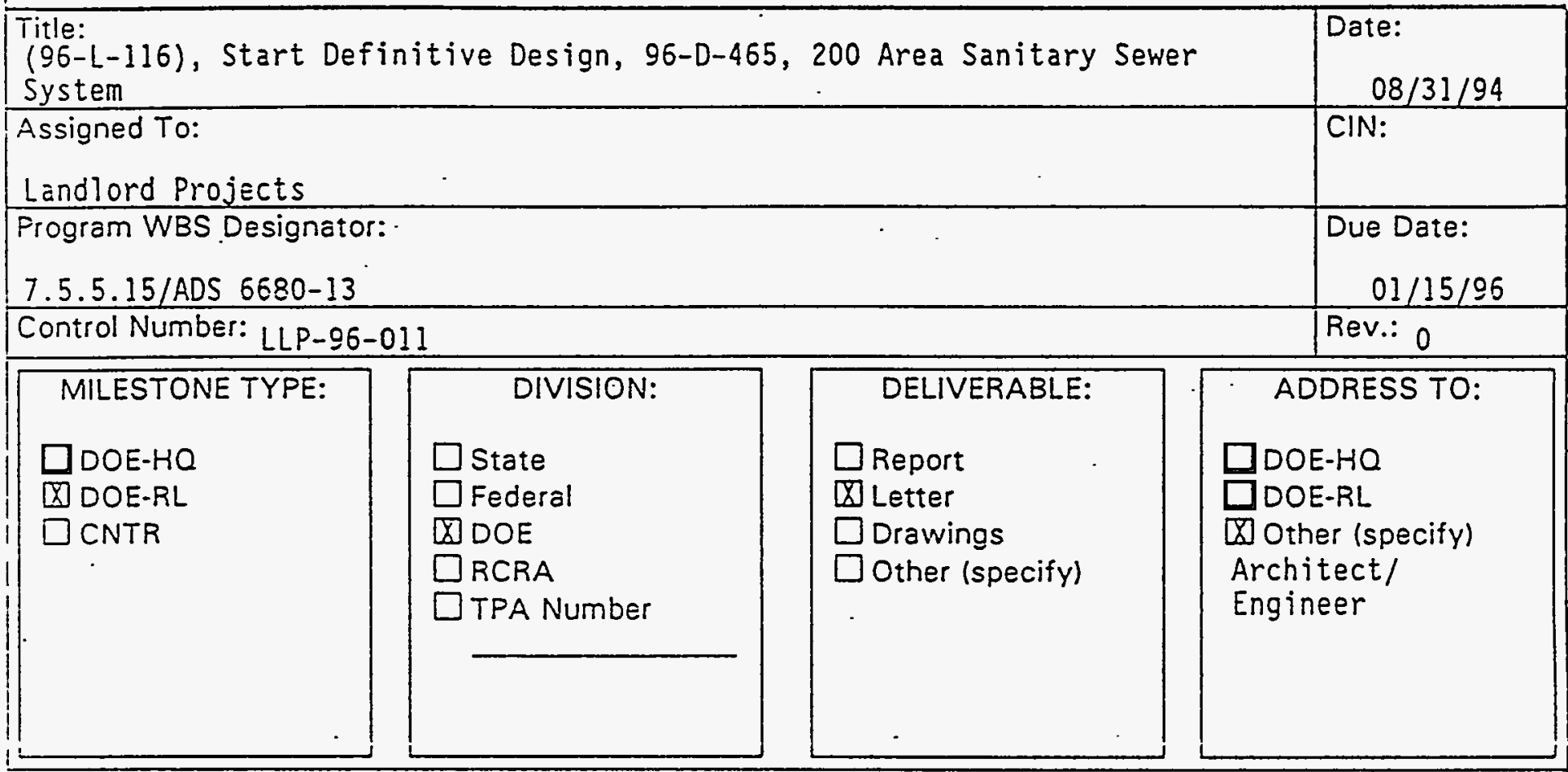

Milestone description:

Start definitive design.

Description of what constitutes completion of this milestone:

Issue letter of instruction to architect/engineer requesting definitive design start.

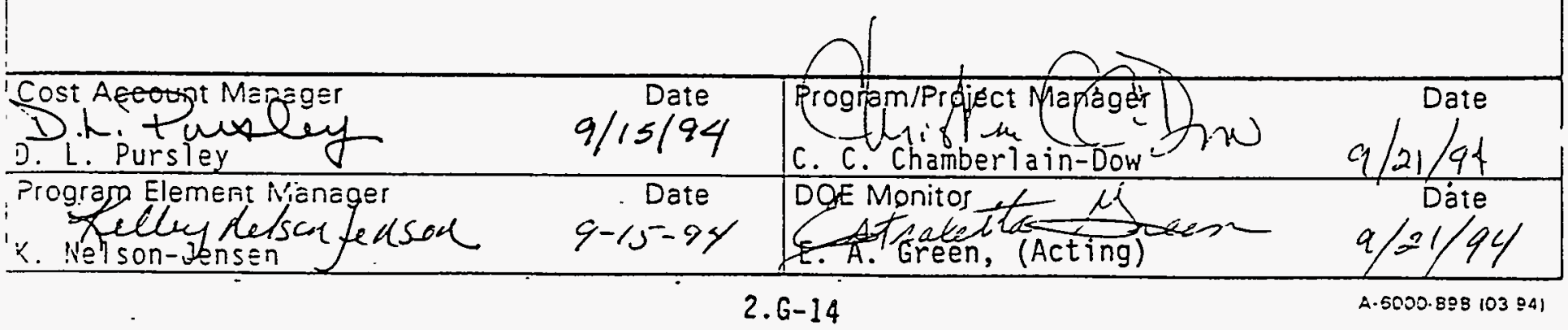


$2 G$.

\section{ICF Kaiser Hanford Company}

MILESTONE DESCRIPTION SHEET

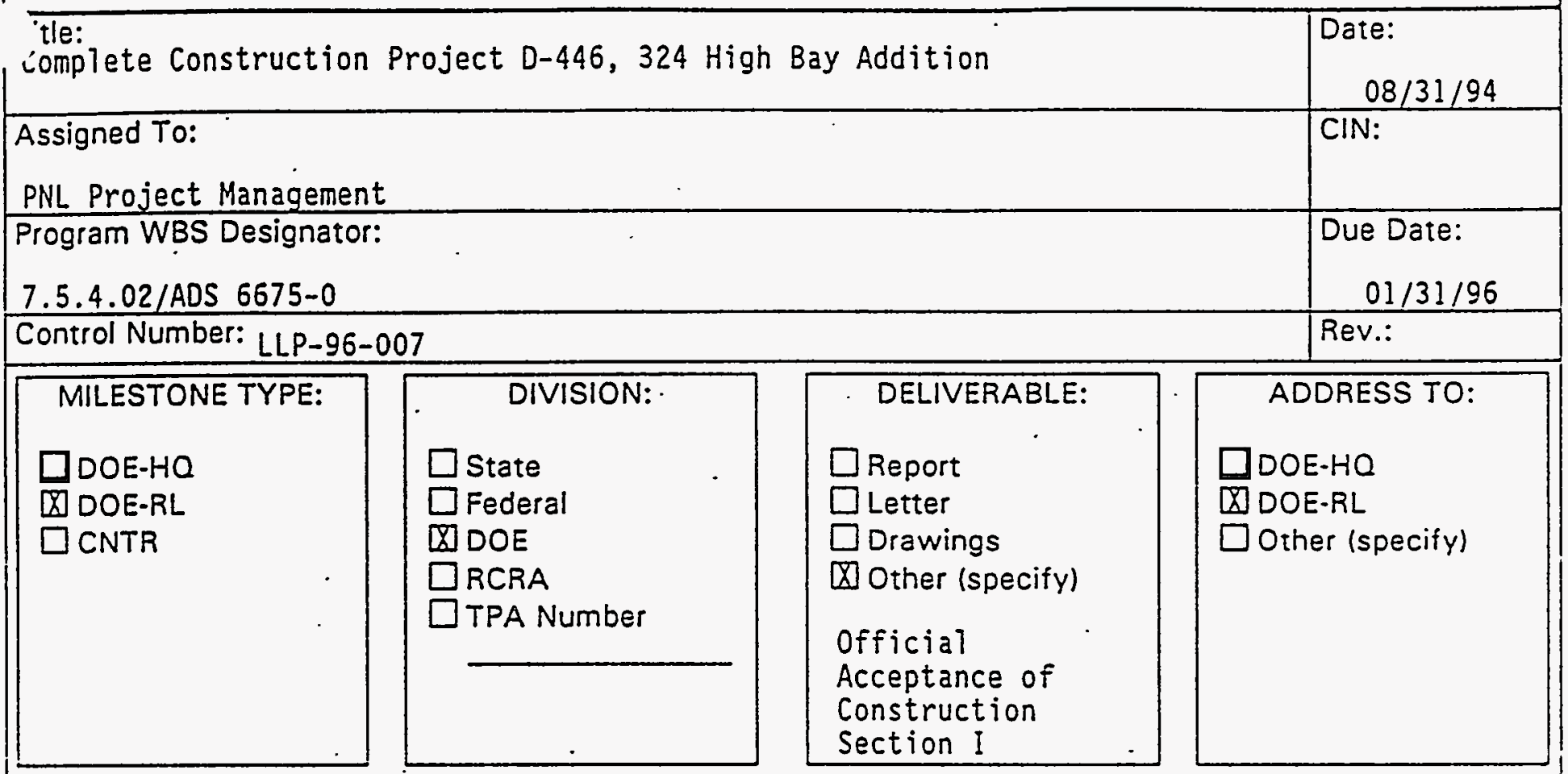

\section{Milestone description:}

Construction is completed and accepted per the design documents.

Description of what constitutes completion of this milestone:

This milestone is attained when Section I of the official Acceptance of

Construction is approved by DOE-RL.

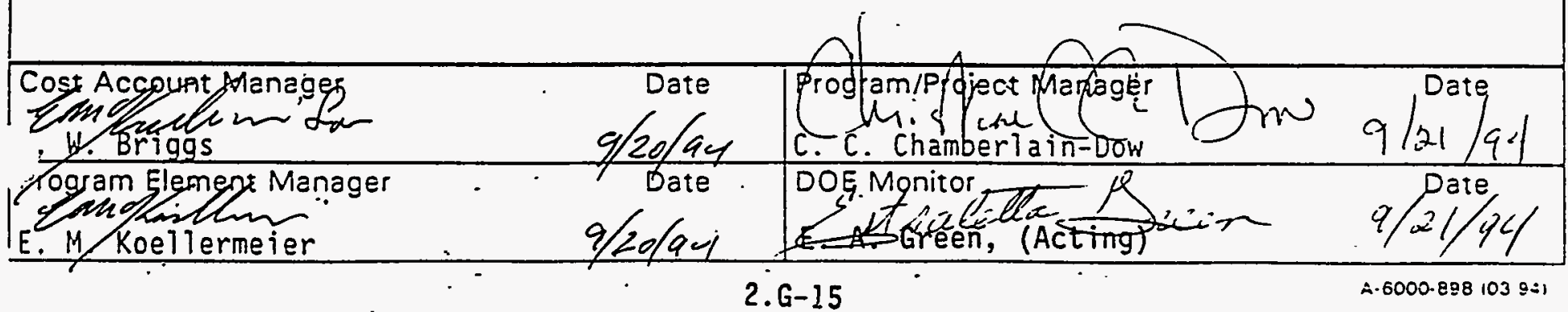


$2 G$.

ICF Kaiser Hanford Company .

MILESTÓNE DESCRIPTION SHEET

Title:

(95-L-102), Start Definitive Design, 95-D-453, Primary Highway Route North

of Wye Barricade

Assigned To:

Landiord Projects

Program WBS Designator:

7.5.5.10/ADS 6680-8

Control Number: LLP-96-010

\begin{tabular}{|c|c|c|}
\hline $\begin{array}{l}\text { MILESTONE TYPE: } \\
\square \text { DOE-HQ } \\
\square \text { DOE-RL } \\
\square \text { CNTR }\end{array}$ & $\begin{array}{l}\text { DIVISION: } \\
\square \text { State } \\
\square \text { Federal } \\
\square \text { DOE } \\
\square \text { RCRA } \\
\square \text { TPA Number }\end{array}$ & $\begin{array}{l}\text { DELIVERABLE: } \\
\square \text { Report } \\
\square \text { Letter } \\
\square \text { Drawings } \\
\square \text { Other (specify) }\end{array}$ \\
\hline
\end{tabular}

DELIVERABLE:

ADDRESS TO:

$\square$ DOE-HO

DDOE-RL

[X] Other (specify)

Architect/

Engineer

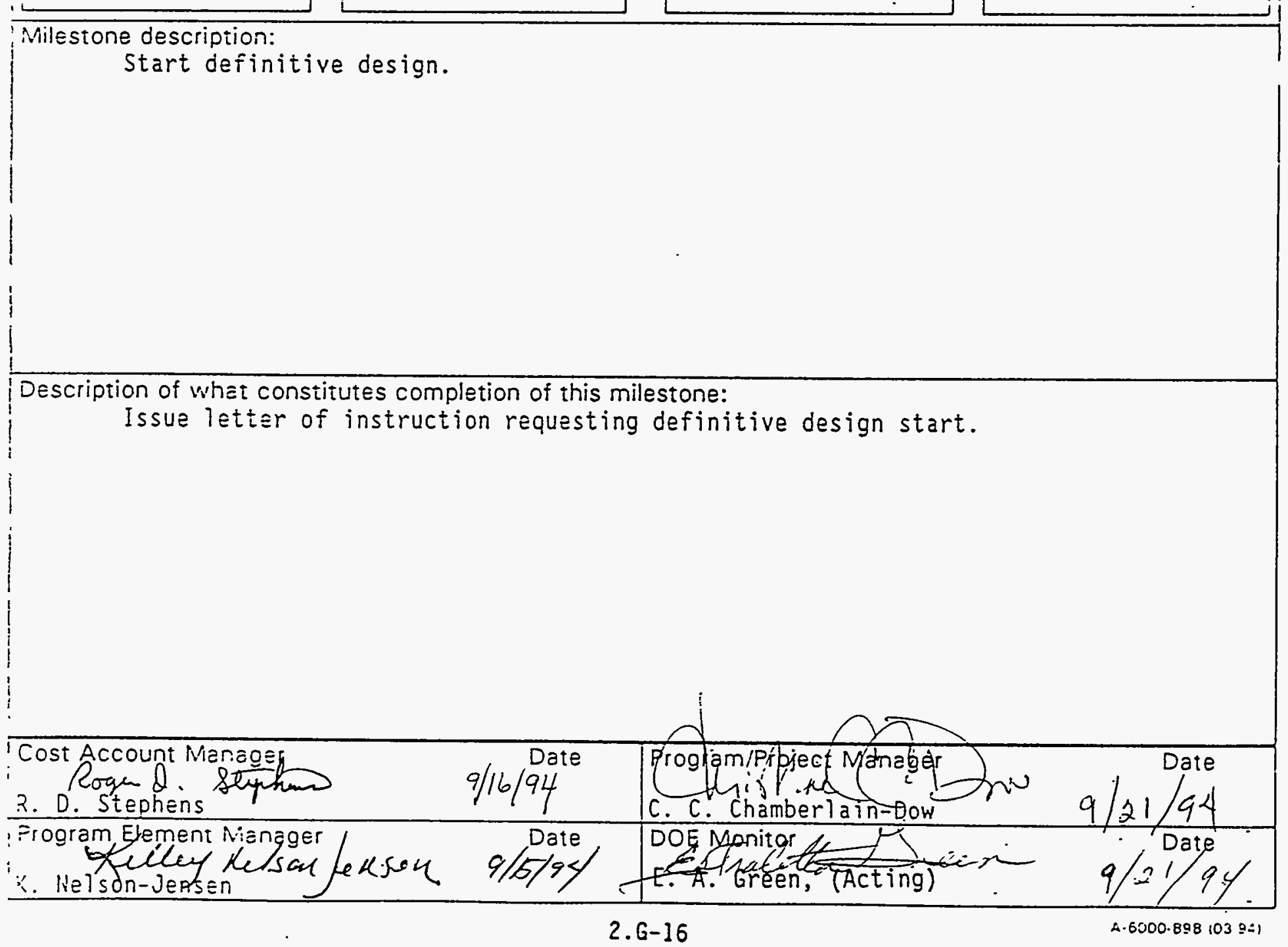


$2 G$.

ICF Kaiser Hanford Company

MILESTONE DESCRIPTION SHEET

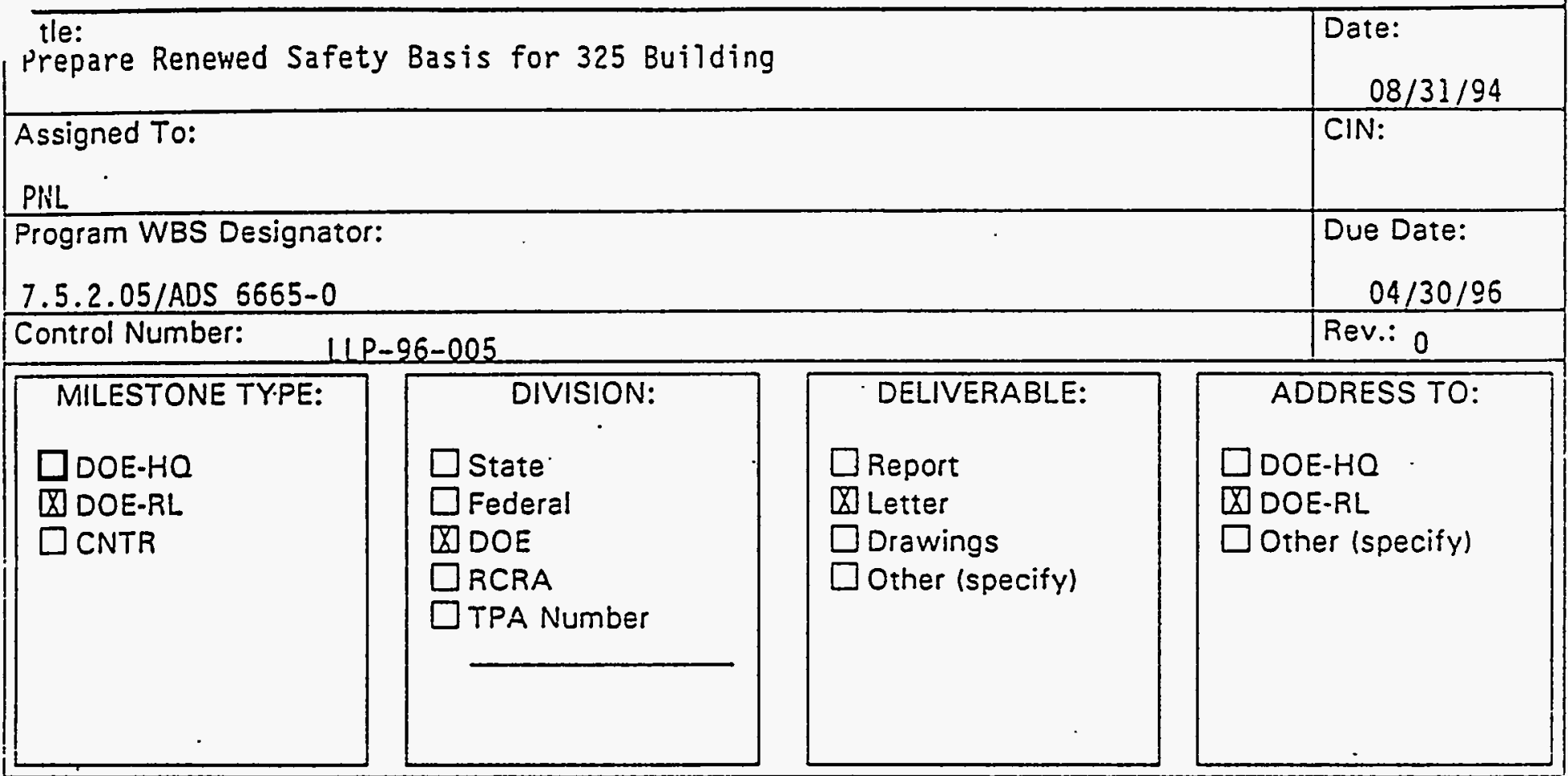

Milestone description:

Prepare renewed safety basis for 325 Building in support of preparing

updated facility Technical Safety Requirements and Safety Analysis Reports.

Start Date: $02 / 28 / 95$

Description of what constitutes completion of this milestone:

Prepare renewed safety basis in accordance with PNL's "Transition PIan for

Achieving Compliance with DOE 5480.22 and DOE 5480.23," Revision 1, August

1993. Obtain PNL independent technical reviews and resolve comments. Obtain approval irom PNL's Safety Review Council. Submit document to DOE-RL for approval.

Cost Account Mianager Date Frograp/Project Mangagé

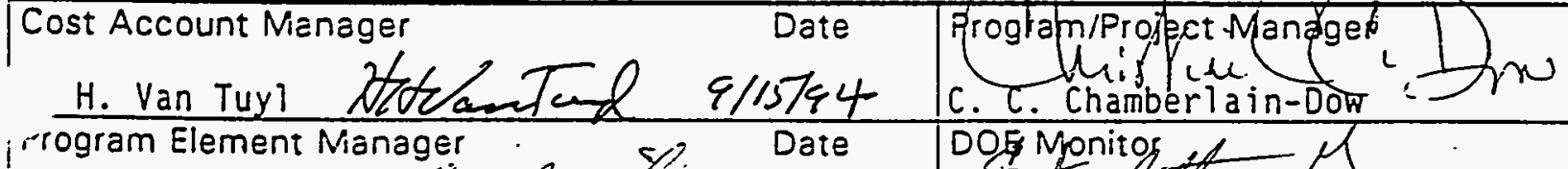
E. M. Koellermeier - I/:3

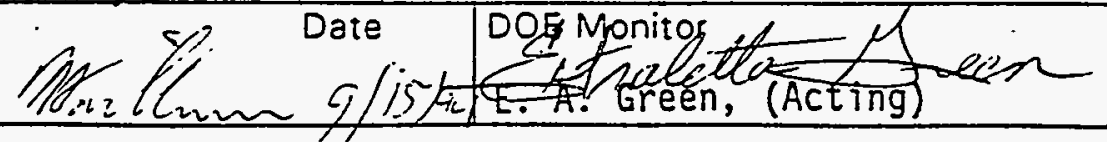


$2 G$.

ICF Kaiser Hanford Company

MILESTONE DESCRIPTION SHEET

Title:

Submit Short Form Data Sheets for FY 2000 Candidate Line Items

Assigned To:

Landlord Program Office

Frogram WBS Designator:

7.5.5.01/ADS 6680-0

Control Number:

$11 \mathrm{P}-96-008$

\begin{tabular}{|c|c|c|c|}
\hline $\begin{array}{l}\text { MILESTONE TYPE: } \\
\square \text { DOE-HO } \\
\text { 囚 DOE-RL } \\
\square \text { CNTR }\end{array}$ & $\begin{array}{l}\text { DIVISION: } \\
\square \text { State } \\
\square \text { Federal } \\
\text { DDOE } \\
\square \text { RCRA } \\
\square \text { TPA Number }\end{array}$ & $\begin{array}{l}\text { DELIVERABLE: } \\
\square \text { Report } \\
\square \text { Letter } \\
\square \text { Drawings } \\
\square \text { Other (specify) } \\
\text { Short Form Data } \\
\text { Sheet }\end{array}$ & $\begin{array}{l}\text { ADDRESS TO: } \\
\square \text { DOE-HO } \\
\text { D DOE-RL } \\
\square \text { Other (specify) }\end{array}$ \\
\hline
\end{tabular}

Milestone description:

Transmit Short Form Data Sheets for FY 2000 Candidate Line Items to DOE-RL.

Description of what constitutes completion of this milestone:

Issue transmittal letter to DOE-RL with completed Short Form Data Sheets for FY 2000 Candidate Line Item projects. 
$2 G$

ICF Kaiser Hanford Company

MILESTONE DESCRIPTION SHEET

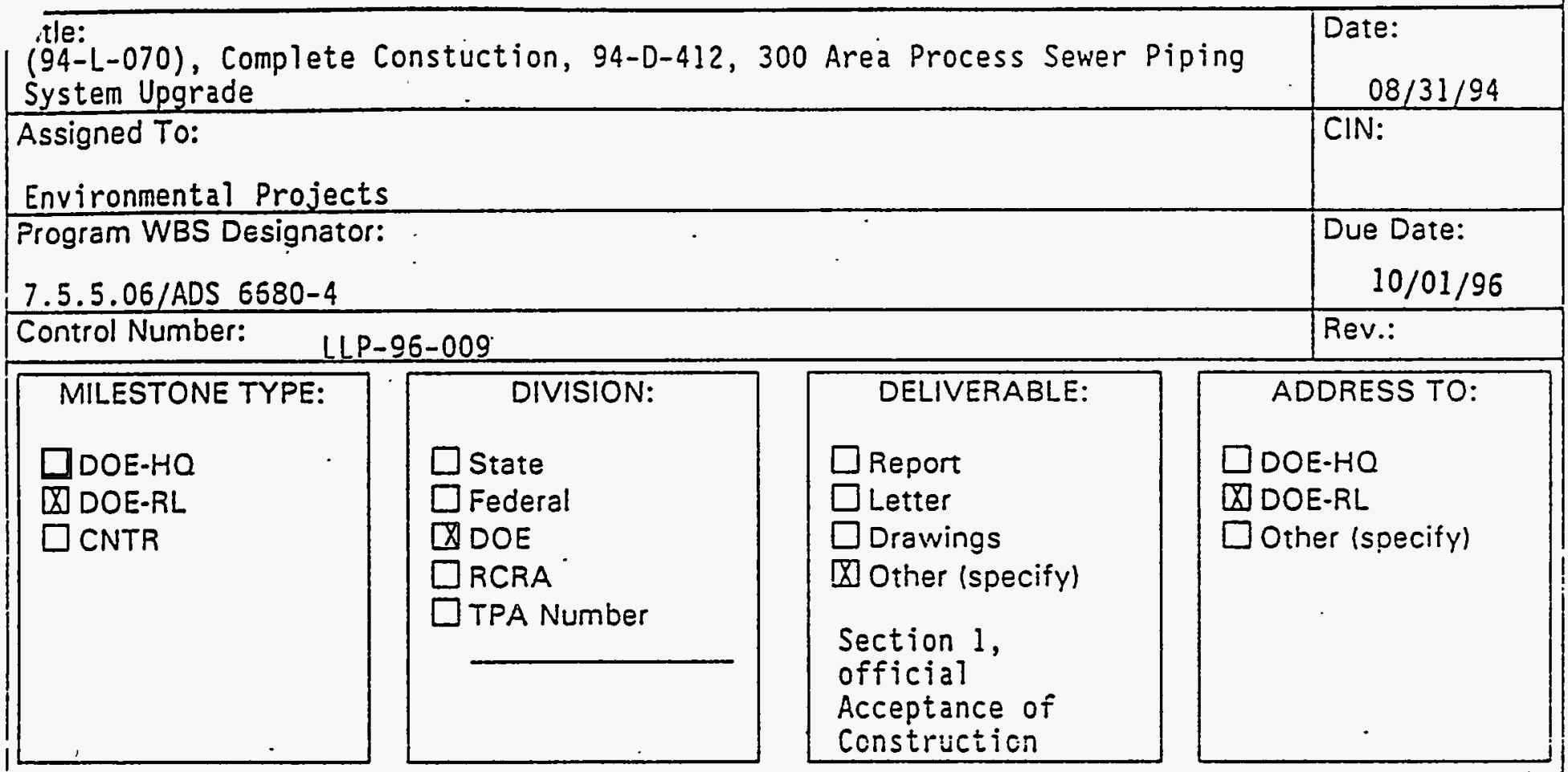

Milestone description:

Complete Construction.

Description of what constitutes completion of this milestone:

DOE-RL, WHC, and ICF KH approval of Official Acceptance of Construction

Section 1.

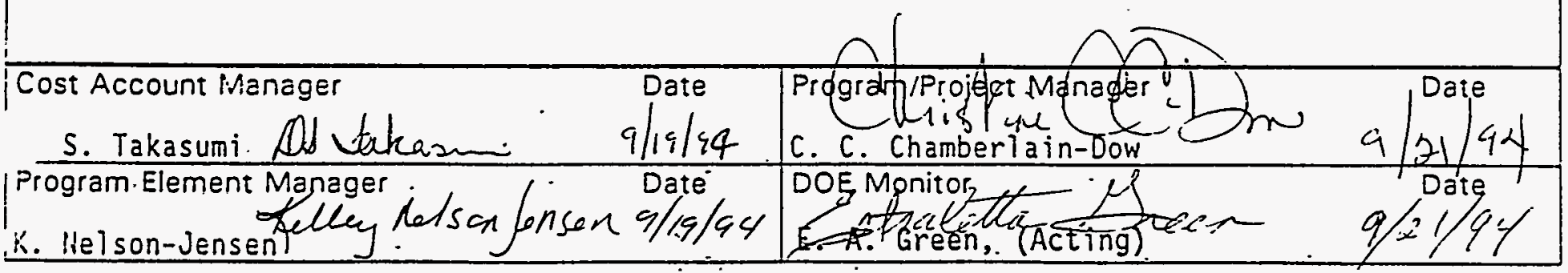




\begin{tabular}{|c|c|c|c|}
\hline \multicolumn{4}{|c|}{$\begin{array}{l}\text { ICF Kaiser Hanford Company } \\
\text { MILESTONE DESCRIPTION SHEET }\end{array}$} \\
\hline \multicolumn{3}{|c|}{$\begin{array}{l}\text { Title: } \\
\text { Complete Renewed Safety Basis for } 327 \text { Building }\end{array}$} & $\begin{array}{l}\text { Date: } \\
08 / 31 / 94\end{array}$ \\
\hline \multicolumn{3}{|l|}{$\begin{array}{l}\text { Assigned To: } \\
\text { PNL }\end{array}$} & CIN: \\
\hline \multicolumn{3}{|c|}{ Frogram WBS Designator: } & \begin{tabular}{|l} 
Due Date: \\
$12 / 31 / 96$
\end{tabular} \\
\hline \multicolumn{3}{|c|}{ Control Number: $\quad$ LLP-97-005 } & Rev.: 0 \\
\hline $\begin{array}{l}\text { MILESTONE TY.PE: } \\
\square \text { DOE-HQ } \\
\text { 囚 DOE-RL } \\
\square \text { CNTR }\end{array}$ & $\begin{array}{l}\text { DIVISION: } \\
\square \text { State } \\
\square \text { Federal } \\
\text { 囚DOE } \\
\square \text { RCRA } \\
\square \text { TPA Number }\end{array}$ & $\begin{array}{l}\text { DELIVERABLE: } \\
\square \text { Report } \\
\square \text { Letter } \\
\square \text { Drawings } \\
\square \text { Other (specify) }\end{array}$ & $\begin{array}{l}\text { ADDRESS TO: } \\
\square \text { DOE-HO } \\
\text { Q DOE-RL } \\
\square \text { Other (specify) }\end{array}$ \\
\hline
\end{tabular}

Milestone description:

Complete renewed safety basis for 327 Building in support of preparing updated facility Technical Safety Requirements and Safety Analysis Reports.

Start Date:04/30/95

Description of what constitutes completion of this milestone:

Perform Preliminary Hazards Analysis to evaluate risks associated with operation of the 327 Building. Select potentially bounding accidents for detailed analysis. Perform detailed analysis of selected analyses and determine risks. Prepare renewed safety basis in accordance with PNL's "Transition PIan for Achieving Compliance with DOE 5480.22 and DOE 5480.23," Revision 1, August 1993. Obtain PNL independent technical reviews and resolve comments. Obtain approval from PNL's Safety Review Council. Submit document to DOE-RL for approval.

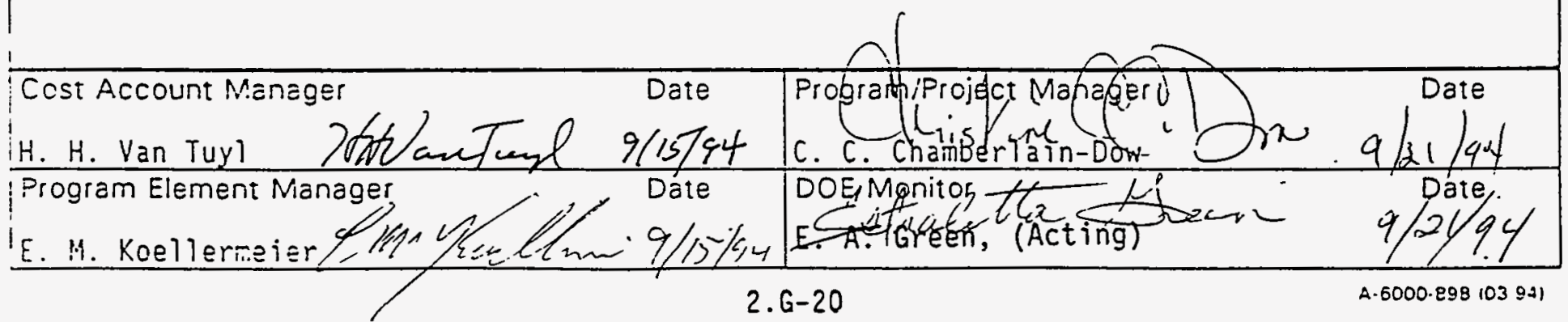


$2 G$.

\section{ICF Kaiser Hanford Company} MILESTONE DESCRIPTION SHEET

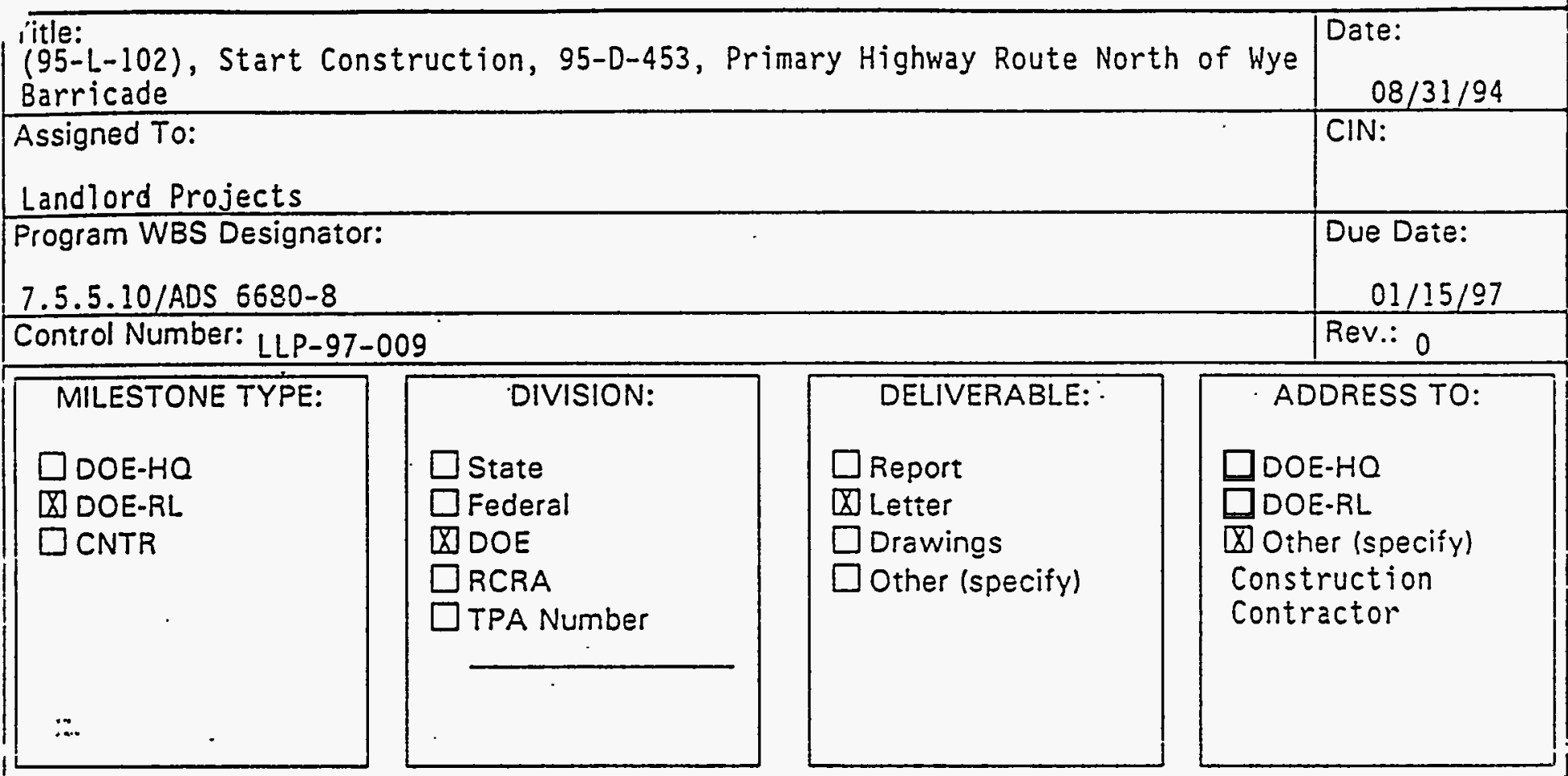

Milestone description:

Start Constuction.

Description of what constitutes completion of this milestone:

Issue letter of instruction to initiate construction activities.

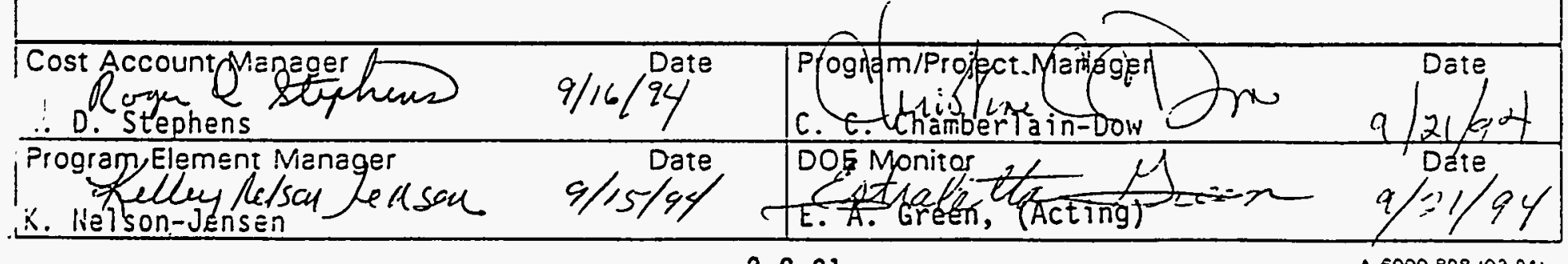


$2 G$.

ICF Kaiser Hanford Company MILESTONE DESCRIPTION SHEE்T

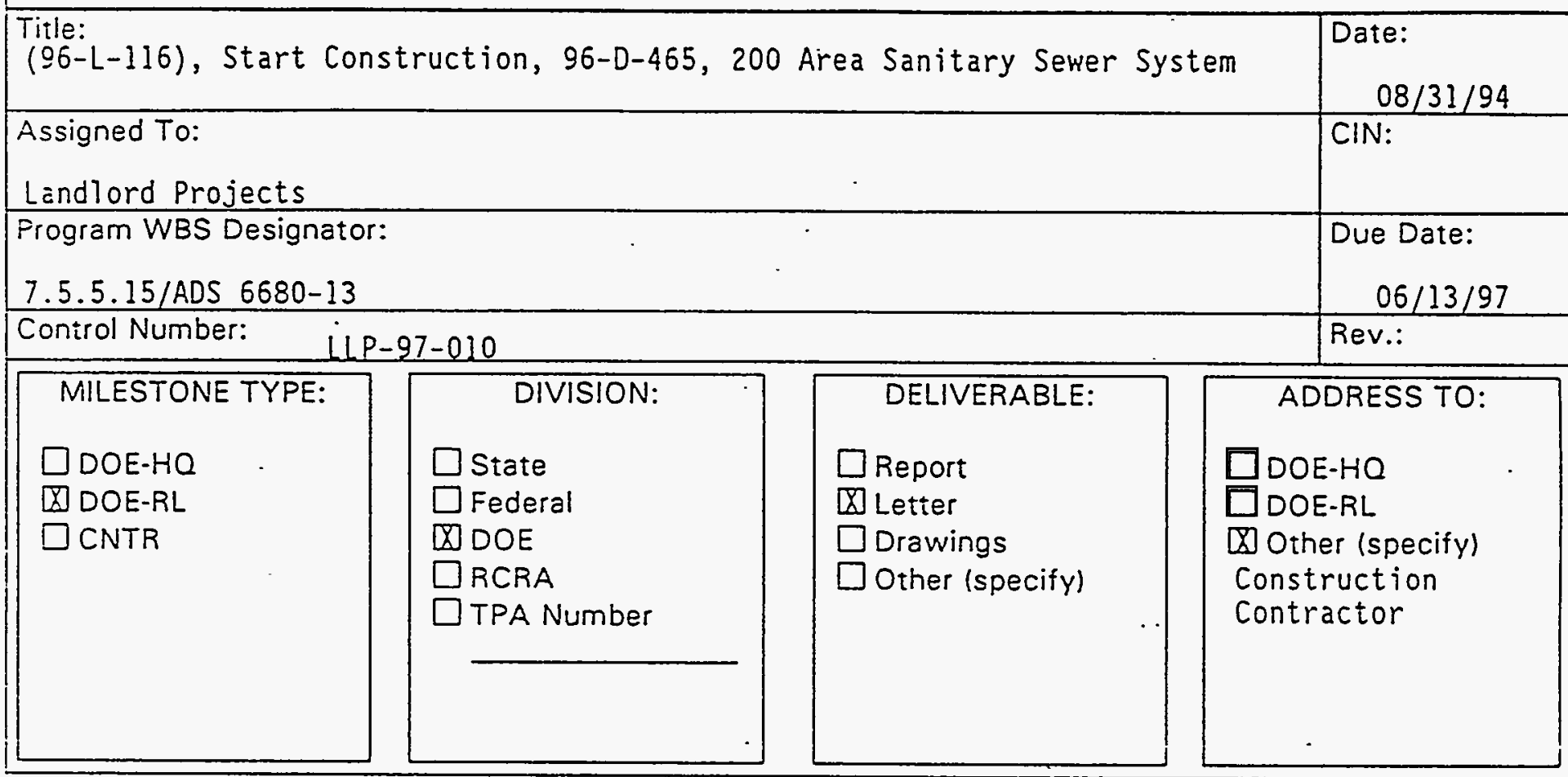

Milestone description:

Start construction.

Description of what constitutes completion of this milestone:

Issue letter of instruction to initiate construction activities.

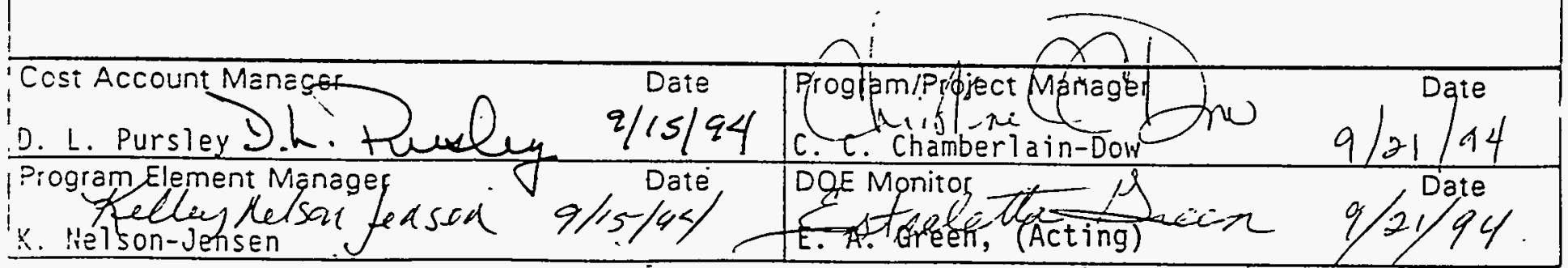


$2 G$.

\section{ICF Kaiser Hanford Company} MILESTONE DESCRIPTION SHEET

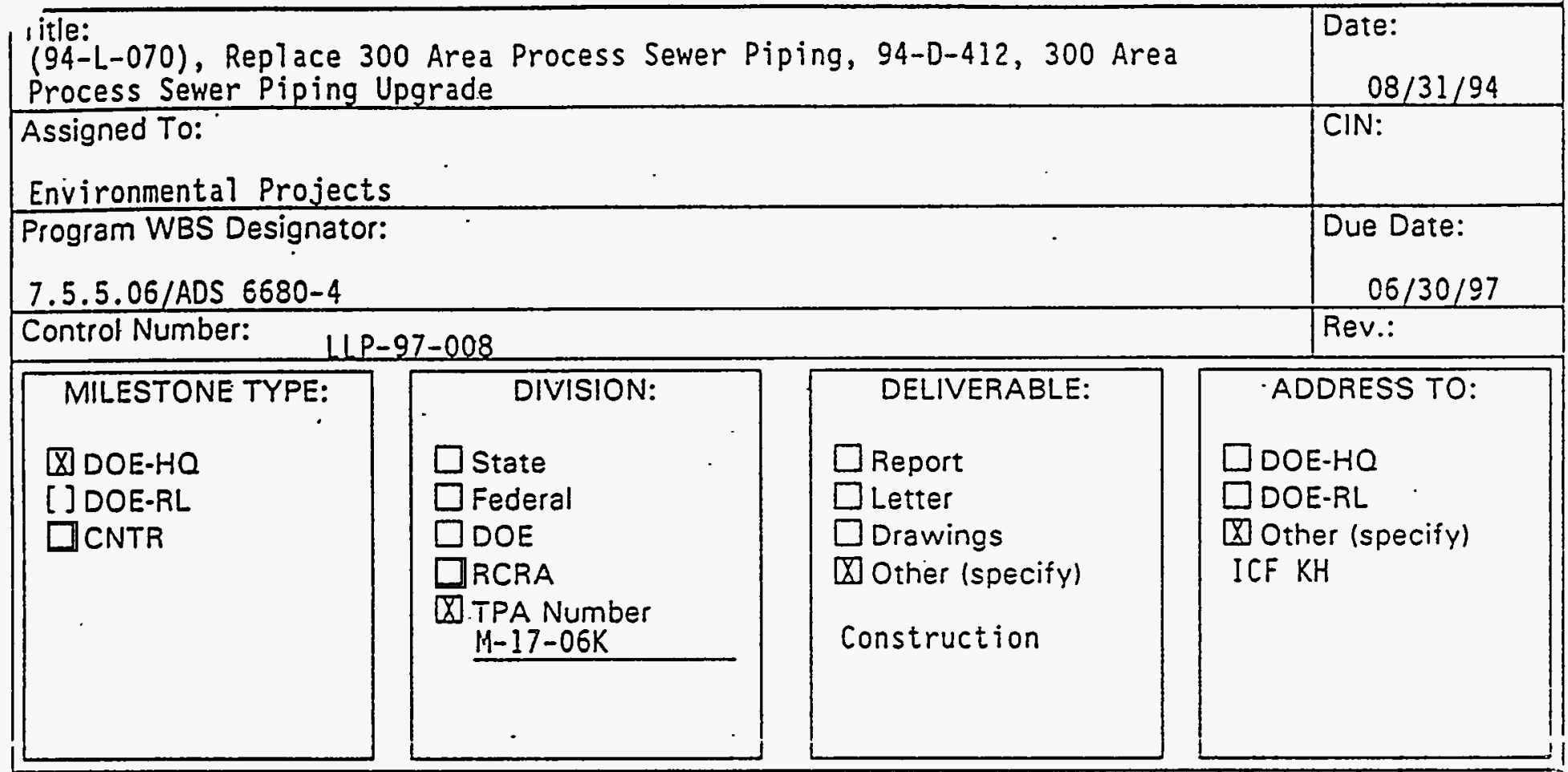

Milestone description:

Replace 300 Area process sewer piping.

\section{Description of what constitutes completion of this milestone:}

Replace 300 Area process sewer piping beginning at five feet outside the buildings and replacing the piping up to interface point into the 300 Area TEDF.

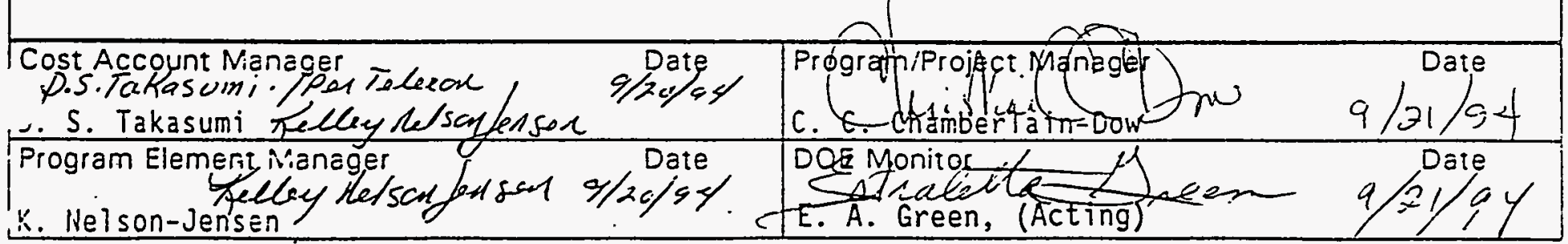


$2 G$.

ICF Kaiser Hanford Company MILESTONE DESCRIPTION SHEETT

Title:

Submit Short Form Data Sheets for FY 2001 Candidate Line Items

Assigned To:

Landlord Program Office

Program WBS Designator:

7.5.5.01/ADS 6680-0

Control Number:

$1 L P-97-007$

\begin{tabular}{|l|l|}
\hline MILESTONE TYPE: & \multicolumn{1}{c|}{ DIVISION: } \\
DDOE-HO \\
$\square$ DOE-RL \\
$\square$ CNTR \\
$\square$ State \\
$\square$ Federal \\
$\square$ DOE \\
$\square$ RCRA \\
$\square$ TPA Number \\
\\
\end{tabular}

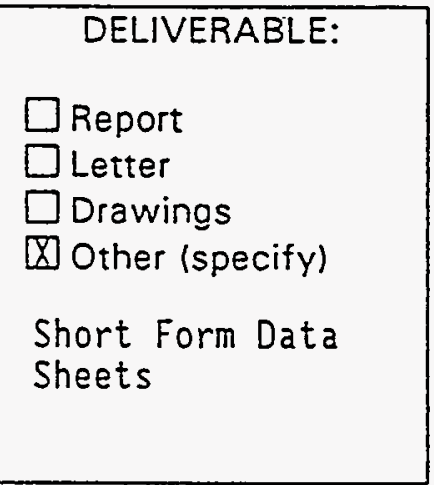

Date:

$08 / 31 / 94$

CIN:

Due Date:

$08 / 01 / 97$

Rev.: 0

Milestone description:

Transmit Short Form Data Sheets for FY 2001 Candidate Line Items to DOE-RL.

Description of what constitutes completion of this milestone:

Issue transmittal letter to DOE-RL with completed Short Form Data Sheets for

FY 2001 Candidate Line Item projects.

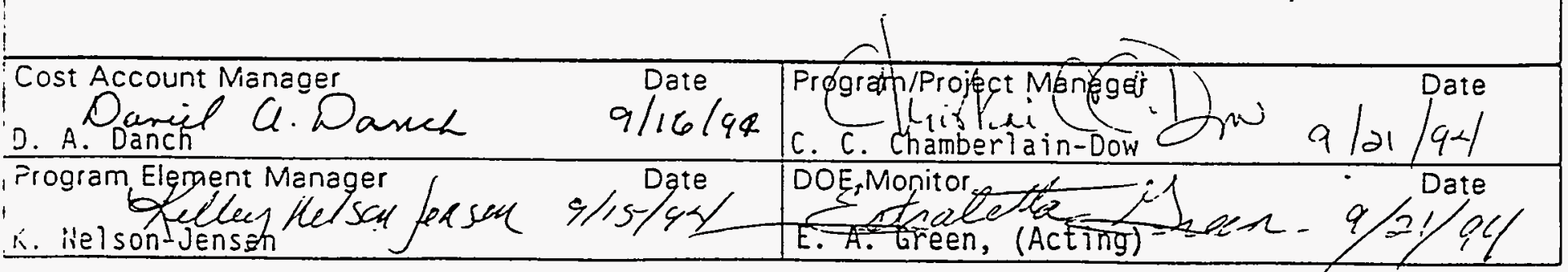




\section{LANDLORD PROGRAM}

7.5

\begin{tabular}{|c|c|c|c|c|c|c|c|c|}
\hline $\begin{array}{c}\text { Program } \\
\text { Element/ADS * }\end{array}$ & $\begin{array}{l}\text { Fund Type } \\
\text { (OP, CE, GPP, } \\
\text { LI, ADS) }\end{array}$ & $\begin{array}{l}\text { FY } 1995 \text { SMS } \\
\text { Guidance }\end{array}$ & $\begin{array}{l}\text { FY } 1996 \\
\text { Target }\end{array}$ & $\begin{array}{l}\text { FY } 1997 \\
\text { Target }\end{array}$ & $\begin{array}{l}\text { FY } 1998 \\
\text { Target }\end{array}$ & $\begin{array}{l}\text { FY } 1999 \\
\text { Target }\end{array}$ & $\begin{array}{r}\text { FY } 2000 \\
\text { Target }\end{array}$ & $\begin{array}{c}\text { FY } 2001 \text { * } \\
\text { Target }\end{array}$ \\
\hline $\begin{array}{l}6660-0 / \text { Program } \\
\text { integration }\end{array}$ & $\begin{array}{l}\text { OP } \\
\text { TOTAL }\end{array}$ & $\begin{array}{l}5070 \\
5070\end{array}$ & $\begin{array}{l}5234 \\
5234\end{array}$ & $\begin{array}{l}6665 \\
6665\end{array}$ & $\begin{array}{l}10677 \\
10677\end{array}$ & $\begin{array}{l}10319 \\
10319\end{array}$ & $\begin{array}{l}11065 \\
11065\end{array}$ & $\begin{array}{l}11397 \\
11397\end{array}$ \\
\hline $\begin{array}{l}6665-0 / \text { Expense } \\
\text { Funded } \\
\text { Projects }\end{array}$ & $\begin{array}{l}\text { OP } \\
\text { TOTAL }\end{array}$ & $\begin{array}{l}7427 \\
7427\end{array}$ & $\begin{array}{l}8930 \\
8930\end{array}$ & $\begin{array}{l}96156 \\
16156\end{array}$ & $\begin{array}{l}45792 \\
45792\end{array}$ & $\begin{array}{l}46962 \\
46962\end{array}$ & $\begin{array}{l}49028 \\
49028\end{array}$ & $\begin{array}{l}50499 \\
50499\end{array}$ \\
\hline $\begin{array}{l}\text { 6670-0/Capital } \\
\text { Equipment }\end{array}$ & $\begin{array}{l}\text { OP } \\
\text { CE } \\
\text { TOTAL }\end{array}$ & $\begin{array}{r}0 \\
13912 \\
13912\end{array}$ & $\begin{array}{r}0 \\
9360 \\
9466\end{array}$ & $\begin{array}{r}0 \\
6088 \\
6197\end{array}$ & $\begin{array}{r}0 \\
15353 \\
95465\end{array}$ & $\begin{array}{r}0 \\
10877 \\
10992\end{array}$ & $\begin{array}{r}0 \\
15496 \\
15615\end{array}$ & $\begin{array}{r}0 \\
15960 \\
15960\end{array}$ \\
\hline $\begin{array}{l}6675-0 / \text { General } \\
\text { Plant Projects }\end{array}$ & $\begin{array}{l}\text { OP } \\
\text { GPP } \\
\text { TOTAL }\end{array}$ & $\begin{array}{l}1195 \\
7200 \\
8395\end{array}$ & $\begin{array}{l}1330 \\
7300 \\
8630\end{array}$ & $\begin{array}{l}9294 \\
6150 \\
7444\end{array}$ & $\begin{array}{r}1401 \\
9500 \\
10901\end{array}$ & $\begin{array}{r}1443 \\
9500 \\
10943\end{array}$ & $\begin{array}{r}1487 \\
9500 \\
10987\end{array}$ & $\begin{array}{r}1532 \\
9500 \\
11032\end{array}$ \\
\hline $\begin{array}{l}\text { 6680-0/Line } \\
\text { Items }\end{array}$ & $\begin{array}{l}\text { OP } \\
\text { LI } \\
\text { TOTAL }\end{array}$ & $\begin{array}{l}1216 \\
1216\end{array}$ & $\begin{array}{l}728 \\
728\end{array}$ & $\begin{array}{l}940 \\
7200 \\
8140\end{array}$ & $\begin{array}{r}1398 \\
12050 \\
13448\end{array}$ & $\begin{array}{r}1570 \\
18800 \\
20370\end{array}$ & $\begin{array}{r}1512 \\
27500 \\
29012\end{array}$ & $\begin{array}{r}1557 \\
37000 \\
38557\end{array}$ \\
\hline $\begin{array}{l}6680-1 / 300 \\
\text { Area, Multi } \\
\text { Purpose } \\
\text { Facility } \\
\text { (Canceled) }\end{array}$ & $\begin{array}{l}\text { OP } \\
\text { LI } \\
\text { TOTAL }\end{array}$ & $\begin{array}{r}0 \\
4000 \\
4000\end{array}$ & $\begin{array}{l}200 \\
500 \\
700\end{array}$ & $\begin{array}{l}49 \\
41\end{array}$ & & & & \\
\hline $\begin{array}{l}\text { 6680-2/200 } \\
\text { East Steam } \\
\text { Sys. Rehab., } \\
\text { Ph it }\end{array}$ & $\begin{array}{l}\text { OP } \\
\text { LI } \\
\text { TOTAL }\end{array}$ & $\begin{array}{r}26 \\
600 \\
626\end{array}$ & & & & & & \\
\hline $\begin{array}{l}\text { 6680-3/ } \\
\text { Landlord } \\
\text { Safety Comp.. } \\
\text { Ph I }\end{array}$ & $\begin{array}{l}\text { OP } \\
\text { TOTAL }\end{array}$ & $\begin{array}{l}52 \\
52\end{array}$ & & & & & & • \\
\hline $\begin{array}{l}6680-4 / 300 \\
\text { Area Process } \\
\text { Sewer Piping } \\
\text { Sys Upgrade }\end{array}$ & $\begin{array}{l}\text { OP } \\
\text { LI } \\
\text { TOTAL }\end{array}$ & $\begin{array}{r}284 \\
7800 \\
8084\end{array}$ & $\begin{array}{r}395 \\
1000 \\
1395\end{array}$ & $\begin{array}{l}225 \\
225\end{array}$ & & & & \\
\hline $\begin{array}{l}6680-5 / \text { Hanford } \\
\text { Infrast. } \\
\text { Undgrnd } \\
\text { Storage Tanks }\end{array}$ & $\begin{array}{l}\text { OP } \\
\text { TOTAL }\end{array}$ & $\begin{array}{l}18 \\
18\end{array}$ & & & & & & \\
\hline
\end{tabular}


FY 1995 MYPP

\section{LANDLORD PROGRAM}

7.5

\begin{tabular}{|c|c|c|c|c|c|c|c|c|}
\hline $\begin{array}{c}\text { Program } \\
\text { El ement/ADS \# }\end{array}$ & $\begin{array}{l}\text { Fund Type } \\
\text { (OP, CE, GPP, } \\
\text { LI, ADS) }\end{array}$ & $\begin{array}{l}\text { FY } 1995 \text { SMS } \\
\text { Guidance }\end{array}$ & $\begin{array}{r}\text { FY } 1996 \\
\text { Target }\end{array}$ & $\begin{array}{l}\text { FY } 1997 \\
\text { rarget }\end{array}$ & $\begin{array}{r}\text { FY } 1998 \\
\text { Target }\end{array}$ & $\begin{array}{r}\text { FY } 1999 \\
\text { Target }\end{array}$ & $\begin{array}{r}\text { FY } 2000 \\
\text { Target }\end{array}$ & $\begin{array}{c}\text { FY } 2001 \text { * } \\
\text { Target }\end{array}$ \\
\hline $\begin{array}{l}6680-71 \\
\text { Landlord } \\
\text { safety Comp.. } \\
\text { Ph II }\end{array}$ & $\begin{array}{l}\text { OP } \\
\text { TOTAL }\end{array}$ & $\begin{array}{l}32 \\
32\end{array}$ & & & & & & \\
\hline $\begin{array}{l}\text { 6680-8/Primary } \\
\text { Highway Rte-H. } \\
\text { of Hye } \\
\text { Barricade }\end{array}$ & $\begin{array}{l}\text { OP } \\
\text { LI } \\
\text { TOTAL }\end{array}$ & $\begin{array}{l}.111 \\
2500 \\
2619\end{array}$ & $\begin{array}{r}56 \\
8100 \\
8156\end{array}$ & $\begin{array}{r}59 \\
5400 \\
5459\end{array}$ & $\begin{array}{r}60 \\
4100 \\
4160\end{array}$ & $\begin{array}{l}36 \\
36\end{array}$ & & \\
\hline $\begin{array}{l}6680-9 / 300 \mathrm{~A} \\
\text { Elec. Distr. } \\
\text { Conv.., \& } \\
\text { Safety } \\
\text { Improve., Ph I }\end{array}$ & $\mathrm{OP}$ & & & & & & & \\
\hline $\begin{array}{l}6680-11 / 324 \\
\text { Facility } \\
\text { Compli./Renov. }\end{array}$ & $\begin{array}{l}\text { OP } \\
\text { LI } \\
\text { TOTAL }\end{array}$ & $\begin{array}{r}157 \\
1500 \\
1657\end{array}$ & $\begin{array}{r}38 \\
3500 \\
3538\end{array}$ & $\begin{array}{l}64 \\
800 \\
864\end{array}$ & $\begin{array}{l}35 \\
35\end{array}$ & & & \\
\hline $\begin{array}{l}6680-12 / 325 \\
\text { Facility } \\
\text { Compli/Renov. }\end{array}$ & $\begin{array}{l}\text { OP } \\
\text { LI } \\
\text { TOTAL }\end{array}$ & $\begin{array}{r}12 \\
1000 \\
1012\end{array}$ & $\begin{array}{l}36 \\
36\end{array}$ & & & & & \\
\hline $\begin{array}{l}6680-13 / 200 \mathrm{~A} \\
\text { Sanitary Sewer } \\
\text { System }\end{array}$ & $\begin{array}{l}\text { OP } \\
\text { LI } \\
\text { TOTAL }\end{array}$ & $\begin{array}{l}122 \\
122\end{array}$ & $\begin{array}{r}71 \\
1800 \\
1871\end{array}$ & $\begin{array}{r}55 \\
4000 \\
4055\end{array}$ & $\begin{array}{r}92 \\
14200 \\
14292\end{array}$ & $\begin{array}{r}85 \\
13400 \\
13485\end{array}$ & & \\
\hline $\begin{array}{l}6680-14 / \\
\text { Hanford Fire } \\
\text { Depart. Fac. }\end{array}$ & $\begin{array}{l}\text { OP } \\
\text { LI } \\
\text { TOTAL }\end{array}$ & $\begin{array}{l}142 \\
142\end{array}$ & $\begin{array}{r}80 \\
1900 \\
1980\end{array}$ & $\begin{array}{r}80 \\
11600 \\
11680\end{array}$ & $\begin{array}{r}200 \\
10100 \\
10300\end{array}$ & $\begin{array}{r}65 \\
6100 \\
6165\end{array}$ & & \\
\hline
\end{tabular}


FY 1995 MYPP

\section{LANDLORD PROGRAM}

7.5

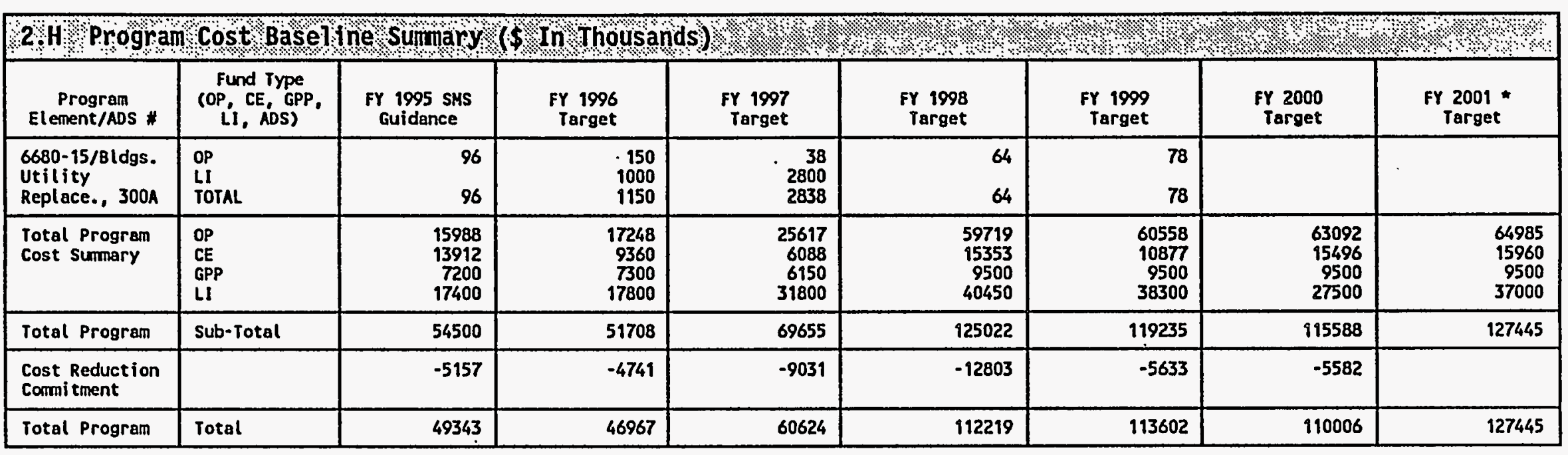

* Assumed 3\% escalation in FY 2001.

N Legend:

ir op - Operating

w CE - Capital Equipment

GPP - General Plant Projects

LI - Line I tems

ADS - Activity Data Sheet 


\section{LANDLORD PROGRAM}

\section{5}

\begin{tabular}{|c|c|c|c|c|}
\hline 2. I Basis of Estimate: & FY 1997 & FY 1996 & FY 1997 \\
\hline Activity/Cost Acct & & & & \\
\hline \multirow{2}{*}{ See below * } & S & & & \\
\hline & FTE & & & \\
\hline
\end{tabular}

Basis of Estimate (Methodology of estimate and justification of scope)

* The Basis of Estimate, i.e., estimate type and estimate risk, was assigned at the task package level, the level at which the work is charged. Task package cost detail summarizes at cost account level providing totals by cost account plan. The basis of estimate can be found on the detailed forms, "Scheduling Activity - Estimating Work Sheet, " retained in a detail estimate file in the Landlord Program Office and is available for review. The Landiord Program RL monitor approved of this format for this section of the MYPP.

There are three estimate types: 1) Budgetary - the cost estimate is based on extrapolations or analogies of other estimates similar in scope; 2) Preliminary - the cost estimate is based on parametric estimating or historical data from a similar project; 3) Definitive - the cost estimate is based on a bottoms up review from takeoff data with known rates or vendor quotes.

The estimate risk is divided into three levels: 1) $\mathrm{H}$ - high risk of estimate fluctuation; 2) M - moderate risk of estimate fluctuation; and 3) L - low risk of estimate fluctuation. 


\section{FY 1995 MYPP}

\section{LANDLORD PROGRAM \\ 7.5}

Program:

Quantify waste by appropriate waste quantity (e.g. cubic meters, gallons, kilograms, etc.) for the EXECUTION YEAR FY 1995 for the following WASTE TYPES for expected waste generation, receipt, transfer, and storage:

\begin{tabular}{|c|c|c|c|c|}
\hline Maste Type & $\begin{array}{c}\text { Maste Generated } \\
\text { (waste unit, e.g., } \\
\text { gallons) }\end{array}$ & $\begin{array}{l}\text { Waste Received } \\
\text { (waste unit, and name of } \\
\text { program received from) }\end{array}$ & $\begin{array}{l}\text { Maste Transferred } \\
\text { (waste unit and name of } \\
\text { program transferred from) }\end{array}$ & $\begin{array}{l}\text { Maste Stored } \\
\text { (waste unit) }\end{array}$ \\
\hline \multicolumn{5}{|l|}{ HLH } \\
\hline \multicolumn{5}{|l|}{ LLW (SOLID) } \\
\hline \multicolumn{5}{|l|}{ LLH (LIOUID) } \\
\hline \multicolumn{5}{|l|}{ LLMH (SOL.ID) } \\
\hline \multicolumn{5}{|l|}{ LLMH (LIRUID) } \\
\hline \multicolumn{5}{|l|}{ LLH (GTC3) } \\
\hline \multicolumn{5}{|l|}{$T R U$} \\
\hline \multicolumn{5}{|l|}{ TRUM } \\
\hline HAZARDOUS & $20 \mathrm{H}^{3}$ & & & \\
\hline SANITARY (LAHDFILL) & $9,000 \mathrm{M}^{3}$ & & & \\
\hline SANITARY (LANDFILL-ASBESTOS) & $75 H^{3}$ & & & \\
\hline
\end{tabular}




\section{LANDLORD PROGRAM}

7.5

FOR THE EXECUTION YEAR - FY 1995 PROVIDE A BRIEF NARRATIVE FOR EACH OF THE FOLLOWING:

PLANS FOR WASTE STORAGE:

The Landlord Program has no plans for storage of wastes in FY 1995.

PLANS FOR INTERPROGRAM WASTE TRANSFER AND/OR DISPOSITION:

Hazardous and sanitary wastes are generated as a result of building demolitions and miscellaneous construction activity. These wastes are transferred to approved disposal sites.

SUMMARY WASTE TYPE ISSUES:

- Adequate onsite disposal facilities are assumed to exist for the majority of sanitary wastes. These are inert wastes that do not require licensed on permitted disposal facilities.

- There is limited disposal space in the onsite licensed sanitary waste landfill. Alternative disposal facilities will have to be developed when the existing landfill reaches capacity. 


\section{Program:}

Quant ify waste by appropriate waste quantity (e.g. cubic meters, gallons, kilograms, etc.) for FY 1996 for the following WASTE TYPES for expected waste generation, receipt, transfer, and storage:

\begin{tabular}{|c|c|c|c|c|}
\hline Haste Type & $\begin{array}{c}\text { Haste Generated } \\
\text { (waste unit, e.g.. } \\
\text { gallons) }\end{array}$ & $\begin{array}{l}\text { Maste Received } \\
\text { (waste unit, and name of } \\
\text { program received from) }\end{array}$ & $\begin{array}{l}\text { Haste Transferred } \\
\text { (waste unit and name of } \\
\text { program transferred from) }\end{array}$ & $\begin{array}{l}\text { Haste Stored } \\
\text { (waste unit) }\end{array}$ \\
\hline \multicolumn{5}{|l|}{ HLW } \\
\hline \multicolumn{5}{|l|}{ LLH (SOLID) } \\
\hline \multicolumn{5}{|l|}{ LLH (LIOUID) } \\
\hline \multicolumn{5}{|l|}{ LLMH (SOLID) } \\
\hline \multicolumn{5}{|l|}{ LLMH (LIQUID) } \\
\hline \multicolumn{5}{|l|}{ LLH (GTC3) } \\
\hline \multicolumn{5}{|l|}{ TRU } \\
\hline \multicolumn{5}{|l|}{ TRUM } \\
\hline HAZARDOUS & $20 \mathrm{M}^{3}$ & & & \\
\hline SANITARY (LANDFILL) & $9,000 \mathrm{~m}^{3}$ & . & & \\
\hline SANITARY (LANDFILL-ASBESTOS) & $75 M^{3}$ & & & \\
\hline
\end{tabular}


FOR THE YEAR FY 1996, PROVIDE A BRIEF NARRATIVE FOR EACH OF THE FOLLOWING:

PLANS FOR WASTE STORAGE:

The Landlord Program has no plans for storage of wastes in FY 1996.

PLANS FOR INTERPROGRAM WASTE TRANSFER AND/OR DISPOSITION:

Hazardous and sanitary wastes are generated as a result of building demolitions and miscellaneous construction activity. These wastes are transferred to approved disposal sites.

SUMMARY WASTE TYPE ISSUES:

- Adequate onsite disposal facilities are assumed to exist for the majority of sanitary wastes. These are inert wastes that do not require licensed on permitted disposal facilities.

- There is limited disposal space in the onsite licensed sanitary waste landfill. Alternative disposal facilities will have to be developed when the existing landfill reaches capacity. 


\section{FY 1995 MYPP}

\section{LANDLORD PROGRAM}

7.5

\section{Program:}

Quantify waste by appropriate waste quantity (e.g. cubic meters, gallons, kilograms, etc:) for Fy 1997 for the following WASTE TYPES for expected waste generation, receipt, transfer, and storage:

\begin{tabular}{|c|c|c|c|c|}
\hline Waste Type & $\begin{array}{l}\text { Haste Generated } \\
\text { (Haste unit, e.g.. } \\
\text { gallons) }\end{array}$ & $\begin{array}{l}\text { Haste Received } \\
\text { (waste unit, and name of } \\
\text { program received from) }\end{array}$ & $\begin{array}{l}\text { Waste Transferred } \\
\text { (waste unit and name of } \\
\text { program transferred from) }\end{array}$ & $\begin{array}{l}\text { Maste Stored } \\
\text { (waste unit) }\end{array}$ \\
\hline \multicolumn{5}{|l|}{ HLH } \\
\hline \multicolumn{5}{|l|}{ LLH (SOLID) } \\
\hline \multicolumn{5}{|l|}{ LL.H (LIQUID) } \\
\hline \multicolumn{5}{|l|}{ LLMH (SOLID) } \\
\hline \multicolumn{5}{|l|}{ LLMH (LIQUTD) } \\
\hline \multicolumn{5}{|l|}{ LLH GTC3 } \\
\hline \multicolumn{5}{|l|}{ TRU } \\
\hline \multicolumn{5}{|l|}{ TRUM } \\
\hline HAZARDOUS & $20 \mathrm{M}^{3}$ & & & \\
\hline SANITARY (LANDFILL) & $9,000 \mathrm{M}^{3}$ & & & \\
\hline SANITARY (LANDFILL-ASBESTOS) & $75 M^{3}$ & & & \\
\hline
\end{tabular}




\section{LANDLORD PROGRAM}

7.5

FOR THE YEAR FY 1997. PROVIDE A BRIEF NARRATIVE FOR EACH OF THE FOLLOWING:

PLANS FOR WASTE STORAGE:

The Landlord Program has no plans for storage of wastes in FY 1997.

PLANS FOR INTERPROGRAM WASTE TRANSFER AND/OR DISPOSITION:

Hazardous and sanitary wastes are generated as a result of building demolitions and miscellaneous construction activity. These wastes are transferred to approved disposal sites.

SUMMARY WASTE TYPE ISSUES:

- Adequate onsite disposal facilities are assumed to exist for the majority of sanitary wastes. These are inert wastes that do not require licensed on permitted disposal facilities.

- The onsite sanitary waste landfill is projected to be full in FY 1997.

- Operation of the facilities constructed by Project 94-D-412, "300 Area Process Sewer Piping System Upgrade," will occur in FY 1997. The piping system will collect liquid hazardous wastes from 300 Area facilities and transport it to the 300 Area Treated Effluent Disposal Facility. 
FY 1995 MYPP

\section{LANDLORD PROGRAM}

7.5

\section{Program:}

Quantify waste by appropriate waste quantity (e.g. cubic meters, gallons, kilograms, etc:) for the years FY 1998 - 2025 for the following WASTE TYPES for expected waste generation, receipt, transfer, and storage:

\begin{tabular}{|c|c|c|c|c|}
\hline Haste Type & $\begin{array}{l}\text { Waste Generated } \\
\text { (waste unit, e.g., } \\
\text { gallons) }\end{array}$ & $\begin{array}{l}\text { Maste Received } \\
\text { (waste unit, and name of } \\
\text { program received from) }\end{array}$ & $\begin{array}{l}\text { Waste Transferred } \\
\text { (waste unit and name of } \\
\text { program transferred from) }\end{array}$ & $\begin{array}{l}\text { Waste Stored } \\
\text { (waste unit) }\end{array}$ \\
\hline HLW & & & s & \\
\hline LLW (SOL.ID) & & & & \\
\hline LLLH (LIQUID) & & & & \\
\hline LLMH (SOLID) & & . & & \\
\hline LLMH (LIOUID) & & & & \\
\hline LLL GTC3 & & & & \\
\hline TRU & & & & \\
\hline TRUM & & & & \\
\hline HAZARDOUS & $540 H^{3}$ & & & \\
\hline SANITARY (LAHDFILL) & $150,000 M^{3}$ & & & \\
\hline SANITARY (LANDFILL-ASBESTOS) & $1,000 \mathrm{~m}^{3}$ & & & \\
\hline
\end{tabular}




\begin{tabular}{|c|c|c|c|c|c|c|c|}
\hline & & & & & & & ןепләјеw \\
\hline & & & & & & & $201 ! 7$ \\
\hline & & & & & & & sts!60/009 \\
\hline & & & & & & & резиашиол!กиз \\
\hline & & & & & & & słs!ฺวบว \\
\hline & $\because$ & औ : : & $\therefore$ & $\because \%$ & & \% & SLSILAIIJS \\
\hline & & & & & 0.91 & $8 \cdot 8$ & د2430 \\
\hline & & & & & $L^{\circ}$ & $21 \cdot 0$ & $130 j \theta 5$ \\
\hline & & & & & & $52 \%$ & 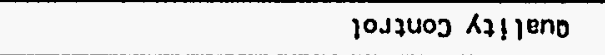 \\
\hline & & & & & 0.2 & $8 \varepsilon^{\circ}$ & 7uB id \\
\hline & & & & & & & 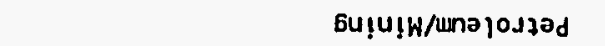 \\
\hline & & & & & $n^{\circ}$ & & Jeכןonk \\
\hline & & & & & $\Sigma .5$ & $99 \cdot 1$ & 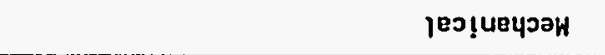 \\
\hline & & & & & $8 \cdot 7$ & $88^{\circ} \mathrm{b}$ & pe!ujsnpul \\
\hline & & & & & $\because \bullet 1$ & 1\%2 & 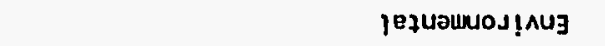 \\
\hline & & & & & $2 \cdot 7$ & $\varepsilon \Sigma: \downarrow$ & 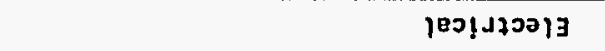 \\
\hline & & & & & $L^{\circ}$ & 0.1 & JajndwoJ \\
\hline & & & & & $6^{\circ}$ & & $1 ! \wedge ! J$ \\
\hline & & & & & & & 1во!шวчग \\
\hline $6 \% 88 \%$ & $848 \%$ & $188 \% ?$ & i. & $295+4 \%$ & 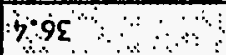 & 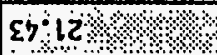 & ঋ \\
\hline & & & & & $s \cdot \mathbf{l}$ & & J24310 \\
\hline & & & & & $s \cdot \varepsilon$ & $6 \Sigma^{\circ} 5$ & 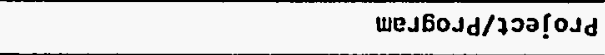 \\
\hline & & & & & & & әА! กпวәха/ןелачวэ \\
\hline & & & & & & $20^{\circ} \mathrm{L}$ & วu!! 2S.!! \\
\hline$s^{4} y 2 \cdot 3$ & $\operatorname{sic}$ & $0 \mathrm{sz} \% \%$ & $0 . \mathrm{s}_{4} \mathrm{Q}$ & $5 \%$ & $0.5 \% \% \%$ & $159,8<$ & 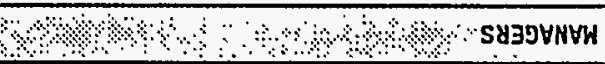 \\
\hline 1002 & 0002 & 6661 & 8661 & 2661 & 9661 & 5661 & RJo5วzeכ qor \\
\hline $14 \%$ & \%ः. & 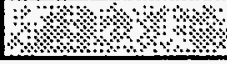 & \%४\%\% & $\because 2$ & \%४ & 1212 & 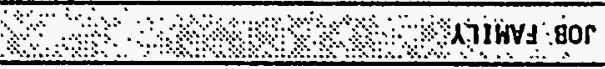 \\
\hline \multicolumn{5}{|c|}{ 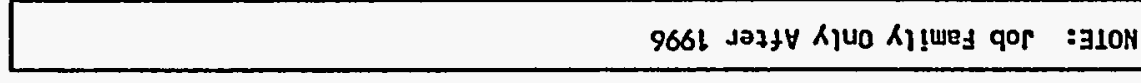 } & & 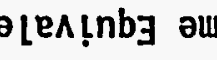 & 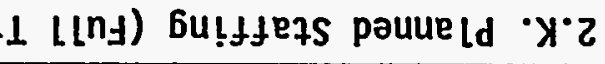 \\
\hline
\end{tabular}

S:L

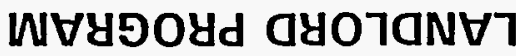

ddAW S66L ᄉJ 


\begin{tabular}{|c|c|c|c|c|c|c|c|}
\hline & & & & & & $50 \div$ & 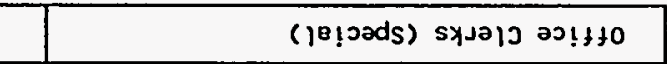 \\
\hline & & & & & 0.2 & $\Sigma 2 \cdot 1$ & 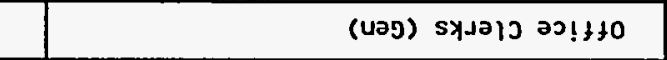 \\
\hline & & & & & & & S7ü7s!ssy u! upy \\
\hline$z^{\prime} \geqslant \varepsilon$ & $2^{\prime}+\sqrt{2}$ & $z^{2}+\varepsilon$ & $2^{2}+\xi \varepsilon$ & $\varepsilon^{* 01}$ & ₹थ2 & 56.05 & 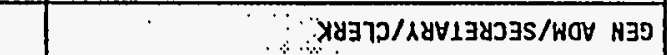 \\
\hline & & & & & 6.9 & $29^{\circ} \mathrm{sl}$ & 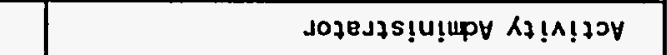 \\
\hline & & & & & & $1 \cdot 2$ & 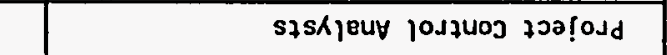 \\
\hline & & & & & & & 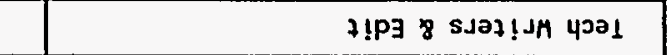 \\
\hline & & & & & & & Ał!」nวas \& p.enfajes \\
\hline & & & & & & & sunN/s!ssళ ue! ’ !s/4d \\
\hline & & & & & & & sue! ! \\
\hline & & & & & & & 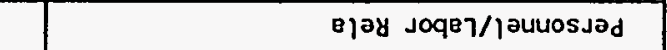 \\
\hline & & & & & & & SJaAme \\
\hline & & & & & & & 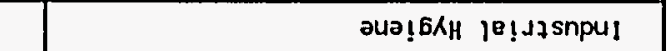 \\
\hline & & & & & & & 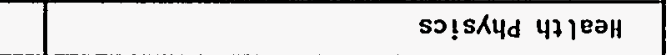 \\
\hline & & & & & $2 \cdot 2$ & 0.6 & 4วS/Jauue/d/2S引 7soJ \\
\hline & & & & & $\Sigma \cdot \downarrow$ & $50^{\circ} z$ & jeut wäs/s dänduoj \\
\hline & & & & & & & 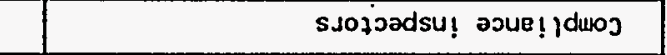 \\
\hline & & & & & & st. 0 & 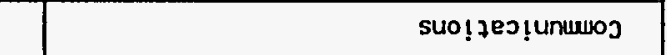 \\
\hline & & & & & $5^{\circ}$ & & 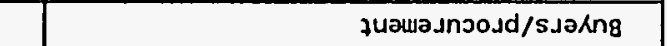 \\
\hline & & & & & & & $2002 ! 40.14$ \\
\hline & & & & & & & J07!pne/zuezunoכวy \\
\hline का & 8:09\%? & $8 \% \angle S$ & $8^{\circ} 09$. & 202 & 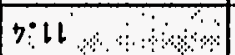 & 56.02 & 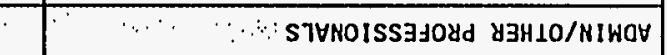 \\
\hline & & & & & & & Јаपзо \\
\hline & & & & & & & jeipos \\
\hline & & & & & & & s7s! I! $5 \times 4 d$ \\
\hline & & & & & & & sue!ว!วешачзен \\
\hline 1002 & 0002 & $666 \mathrm{t}$ & 8661 & 2661 & 9661 & 5661 & Aso5azeo qor \\
\hline & के $\because$ & & & $\therefore$ & 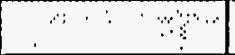 & $\because$ & A7TWV: gor \\
\hline \multicolumn{5}{|c|}{ 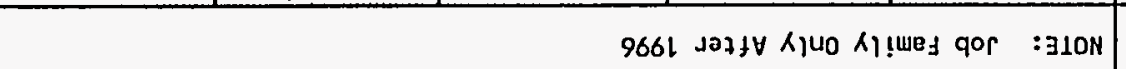 } & \multicolumn{3}{|c|}{ 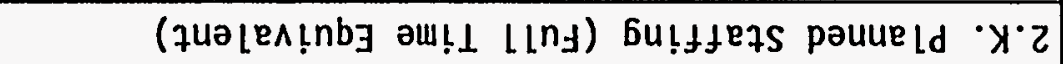 } \\
\hline
\end{tabular}




\section{LANDLORD PROGRAM}

7.5

\begin{tabular}{|c|c|c|c|c|c|c|c|}
\hline \multicolumn{3}{|c|}{ 2.K. Planned Staffing (Full Time Equivalent) } & \multicolumn{5}{|c|}{ NOTE: Job Family Only After 1996} \\
\hline J08 FAMILY YPA & 8\%? & ens & 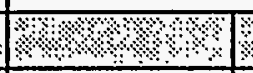 & बिए। & अ.४४ & ए०४ & Qै: \\
\hline Job category & 1995 & 1996 & 1997 & 1998 & 1999 & 2000 & 2001 \\
\hline Secretaries & 4.87 & 4.4 & & & & & \\
\hline \multicolumn{8}{|l|}{ Typist/Hord Process } \\
\hline Student Employee & 3.8 & & & & & & \\
\hline 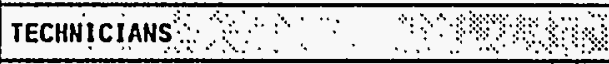 & ४थ:1:1:13 & ब1\% & ४४ & १०००: & ४४४४०: & 18.20 .8 & 0.8 .8 \\
\hline \multicolumn{8}{|c|}{\begin{tabular}{|l|l|l|} 
Computer Oper/Coder & \\
\end{tabular}} \\
\hline Drafters & 0.11 & .4 & & & & & \\
\hline \multicolumn{8}{|l|}{ Engrs/Tech } \\
\hline \multicolumn{8}{|l|}{ Envir. Sci Technicians } \\
\hline Heal th Phys. Technic. & 1.02 & .3 & & & & & \\
\hline \multicolumn{8}{|l|}{ Indus. Saf/Heal th Tech } \\
\hline \multicolumn{8}{|l|}{ Instru/Control Tech } \\
\hline \multicolumn{8}{|l|}{ Lab. Technicians } \\
\hline \multicolumn{8}{|l|}{ Media Technicians } \\
\hline \multicolumn{8}{|l|}{ Survey/Map Tech } \\
\hline \multicolumn{8}{|l|}{ Other } \\
\hline CRAFTS & & $\because 2$ & 8.0 & 145.2 & 145.2 & + 145.2 & 145.2 \\
\hline \multicolumn{8}{|l|}{ Carpenters } \\
\hline Electricians & 0.01 & & & & & & \\
\hline \multicolumn{8}{|l|}{ HVAC } \\
\hline \multicolumn{8}{|l|}{ Machinists } \\
\hline \multicolumn{8}{|l|}{ Masons } \\
\hline \multicolumn{8}{|l|}{ Millurights } \\
\hline \multicolumn{8}{|l|}{ Painters } \\
\hline \multicolumn{8}{|l|}{ Plumbers/Pipefit ters } \\
\hline \multicolumn{8}{|l|}{ Struct/Metal Horkers } \\
\hline Vehic./Mob Equip Mech & & & & & & & \\
\hline
\end{tabular}




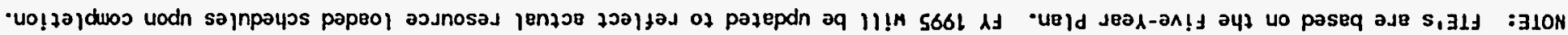

\begin{tabular}{|c|c|c|c|c|c|c|c|}
\hline i。osk & 7ösE $\because$ & $i+87 \varepsilon$ & 2'2ss $\cdots$ & 6.001 & ڤ? & $88 \cdot 09:-1$ & WHyooyd o807any $7 \forall 101$ \\
\hline & & & & & & & دәч70 \\
\hline & & & & & & & spjeng $\alpha_{7}$ ! Jnoas \\
\hline & & & & & & & SJaueajJ/sJoq! luer \\
\hline & & & & & & & כads qej dןән/pueн \\
\hline & & & & & & & uag qeר dןан/риен \\
\hline & & & & & & & Sد2) \\
\hline 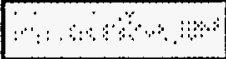 & ४ै। & $\because$, & $\begin{array}{c}\cdots \quad \cdots \\
\cdots\end{array}$ & अध一 & $\therefore \quad$ & कि & 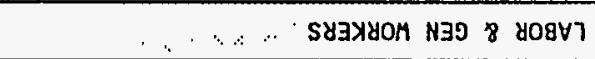 \\
\hline & & & & & & & дачғо \\
\hline & & & & & & & 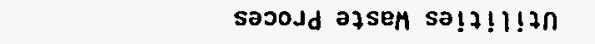 \\
\hline & & & & & & & Zue id Jeajonk \\
\hline & & & & & & & majsks jeכ!wayJ \\
\hline$\therefore$ & $0 \cdot 2 \%$ & $0 \%$ & $0^{\circ} 2$. & $\because \because \cdots$ & $\because \because \cdots: \cdots$ & $\because \because \cdots$ & SYOLYYIdO \\
\hline & & & & & & & Jayto \\
\hline & & & & & & & sıapjay \\
\hline 1002 & 0002 & 6661 & 8661 & 2661 & $966 \mathrm{t}$ & 5661 & 17.06כj8כ qOC \\
\hline$\because "$ & ४य। & $\ldots \ldots$ & $\therefore \cdots+\because ":$ & : : & . & $\because \because$ & 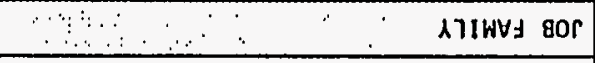 \\
\hline \multicolumn{5}{|c|}{9661 Jaz+V } & \multicolumn{3}{|c|}{ 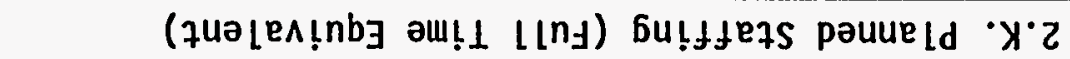 } \\
\hline
\end{tabular}




\section{LANDLORD PROGRAM}

\section{5}

\section{FY 1995 MYPP}

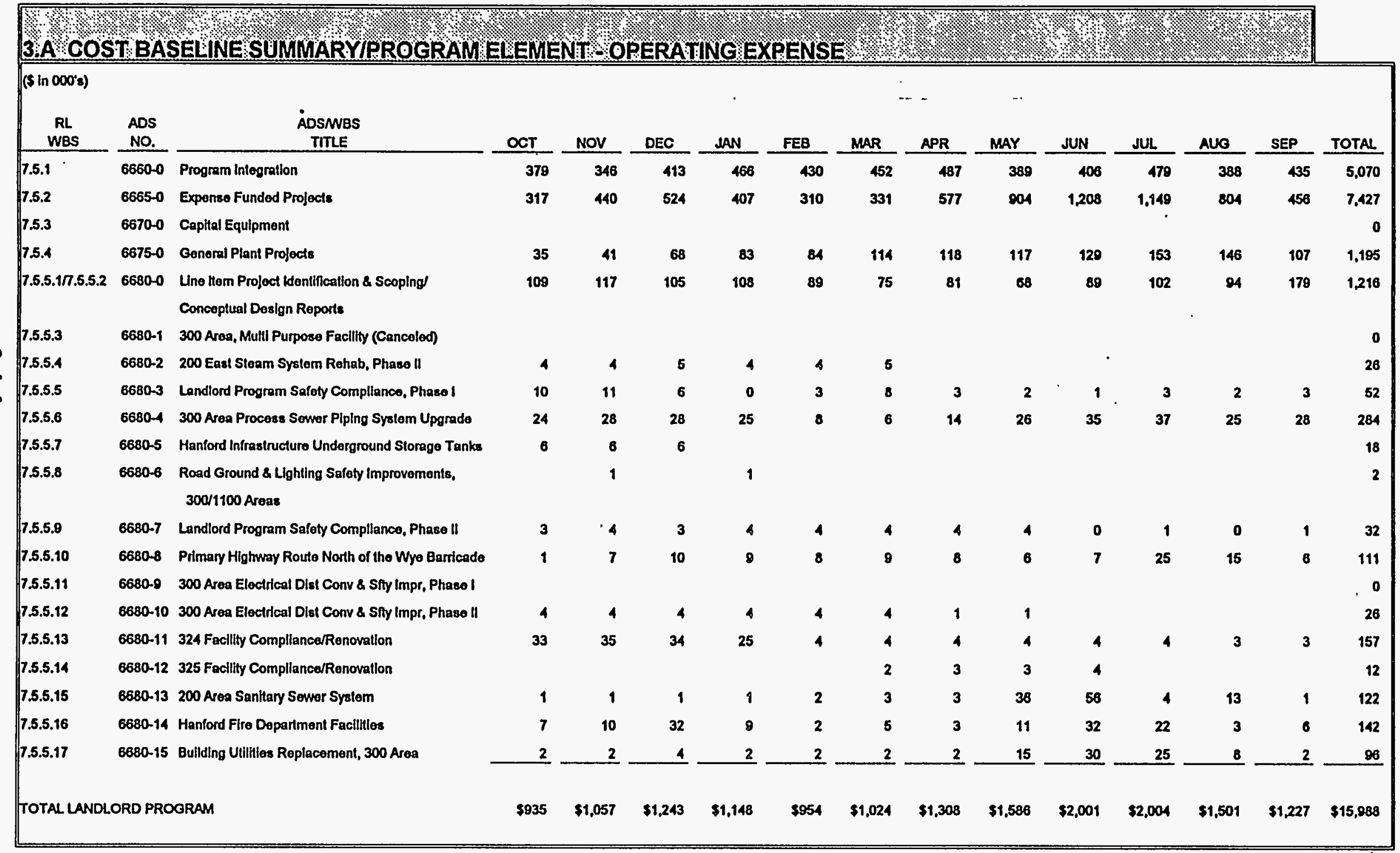




\section{RANDLORD PROGRAM}

\section{5}

\section{FY 1995 MYPP}

\section{A PROGRAM SUMMARY/COSTELEMENT OPERATING EXPENSE}

$\left(\$ \ln 000^{\circ} s\right)$

COST ELEMENT

o. LABOR

1. MATERIALS

2. PURCHASED SERVICES

3. OTHER CONTRACTORS

4- SITE SERVICES

5. INTERNAL CHARGES

6 - IRM

7. DEPARTMENTAL OVERHEAD

8. GAACSP
$\frac{O C T}{423} \frac{\text { NOV }}{400} \frac{\text { DEC }}{445} \frac{\text { JAN }}{413} \frac{\text { FEB }}{329} \frac{\text { MAR }}{381} \frac{\text { APR }}{421} \frac{\text { MAY }}{382} \frac{\text { JUN }}{410}-\frac{\text { JUL }}{393} \frac{\text { AUG }}{324} \frac{\text { SEP }}{355} \frac{\text { TOTAL }}{4,676}$

$\begin{array}{rrrrrrrrrrrrr}1 & 2 & 35 & 32 & 24 & 14 & 7 & 5 & 17 & 27 & 9 & 1 & 174 \\ 68 & 64 & 84 & 94 & 91 & 124 & 203 & 349 & 672 & 639 & 406 & 272 & 3,066\end{array}$

$\begin{array}{llll}68 & 64 & 84 & 94\end{array}$

223

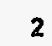

373

4

358

303

276

7

551

520

$\begin{array}{llll}6 & 7 & 7 & 9\end{array}$

$6 \quad 5 \quad 8$

$\begin{array}{lll}73 & 69 & 68\end{array}$

139

167

159

130
12

72 


\section{LANDLORD PROGRAM}

\section{5}

\section{FY 1995 MYPP}

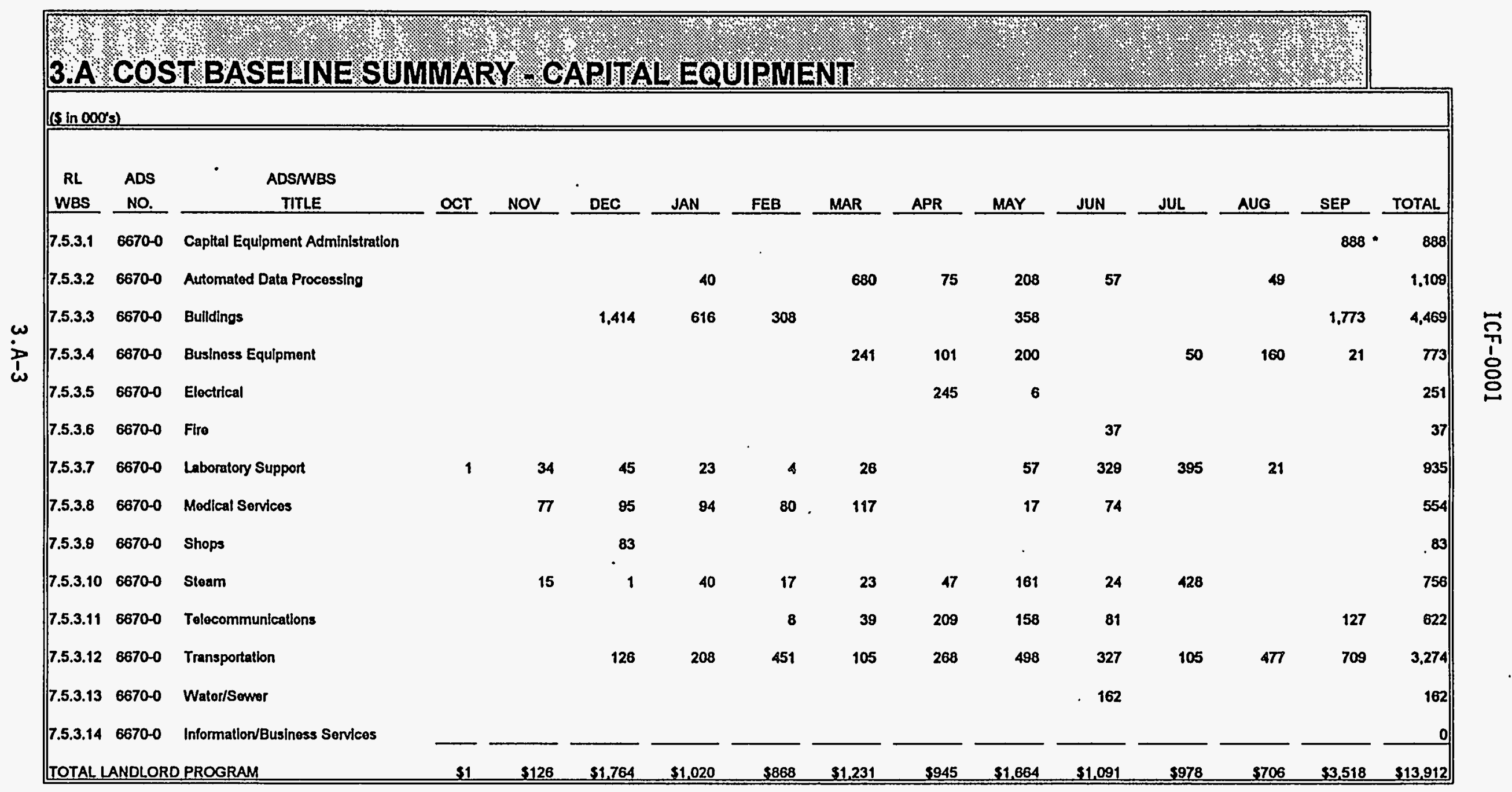

- These funds are for General \& Administrative (G\&A) adder on prior year workscope which will not be completed by yearend. These funds cannot be planned until after yearend. 


\section{LANDLORO PROGRAM}

\section{5}

\section{FY 1995 MYPP}

\section{A COST BASELINE SUMMARY GPP}

\section{( $\$$ in 000's) This illustrates when General Plant Projects will receive Project Authorization for FY 95 funds.}

RL ADS ADSNBS

WBS NO. TITLE

7.5.4 6675-0 General Plant Projects

OCT NOV DEC JAN FEB MAR

APR MAY

JUN

JUL AUG

SEP

TOTAL

$\omega$
$\vdots$
1
+

TOTAL LANDLORD PROGRAM

$\begin{array}{llll}3,350 & 1,100 & 1,700 & 1,050\end{array}$

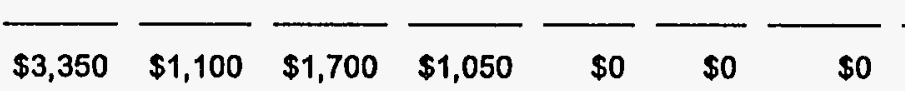

$\$ 0$

$\$ 0$

\$o

\$0

\$0 $\$ 7,200$ 


\section{LANDLORD PROGRAM}

\section{5}

\section{FY 1995 MYPP}

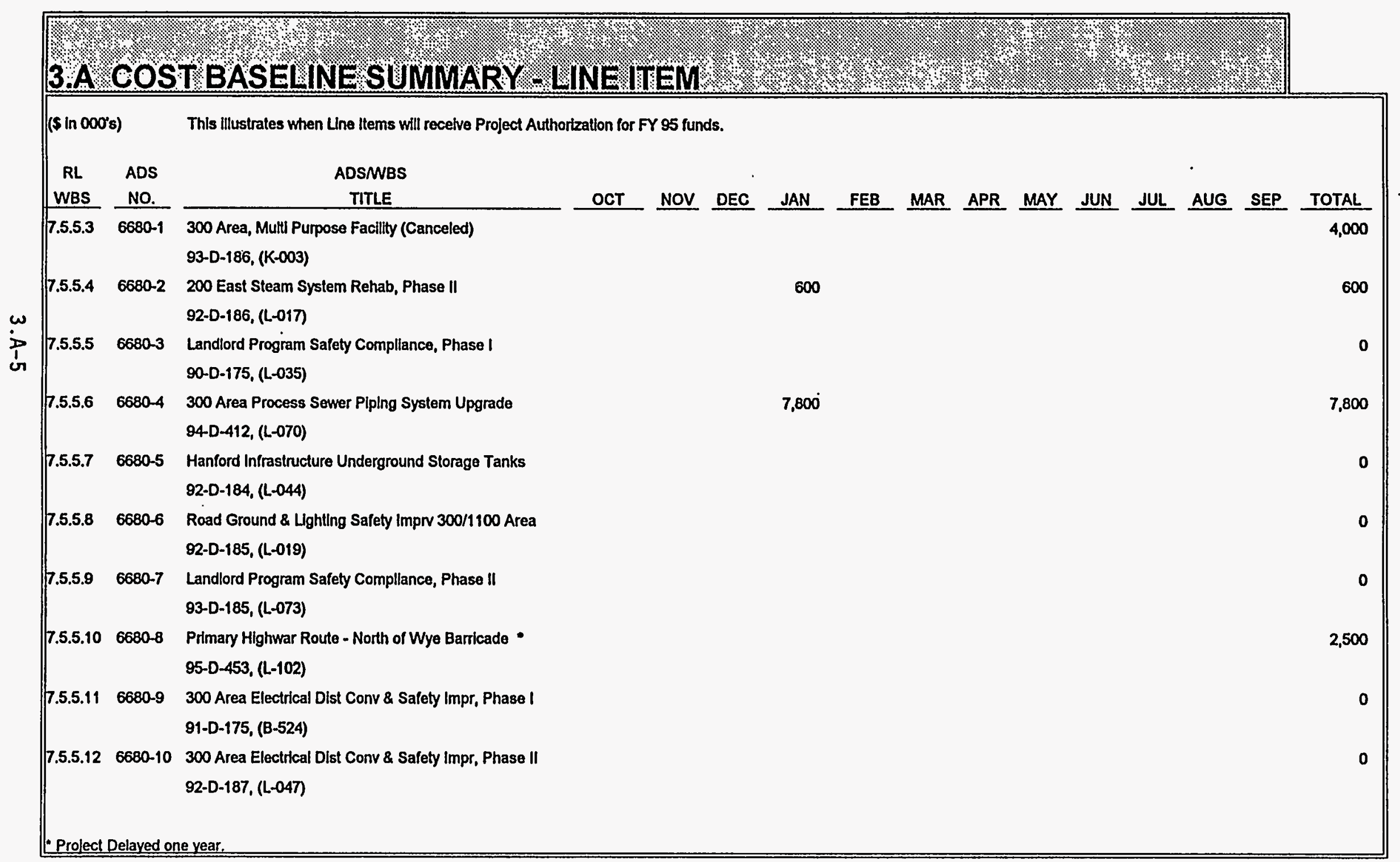




\section{LANDLORD PROGRAM}

\section{5}

\section{FY 1995 MYPP}

\section{A COST BASELINE SUMMARY - LINE ITEM}

( $\$$ in 000's) This Illustrates when Line Items will recelve Project Authorkzation for FY 85 funds.

RL ADS ADSNBS

WBS NO. TITLE

7.5.5.13 6680-11 324 Facility Compllance/Renovation

OCT NOV DEC JAN FEB MAR APR MAY JUN JUL AUG SEP TOTAL 95-D-454, (D-424)

7.5.5.14 6680-12 325 Facility Compllance/Renovation

1,000

1,500

93-D-184, (D-391)

1,000

7.5.5.15 6680-13 200 Area Sanitary Sewer System

96-D-456, (L-116)

7.5.5.16 6680-14 Hanford Fire Department Faclitiles

96-D-466, (L-094)

7.5.5.17 6680-15 Bullding Utillties Replacement, 300 Area

96-D-467, (D-420)

TOTAL LANDLORD PROGRAM

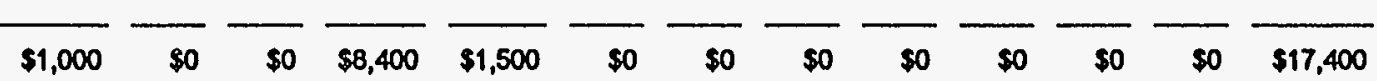




\section{LANDLORD PROGRAM}

\section{5}

\section{FY 1995 MYPP}

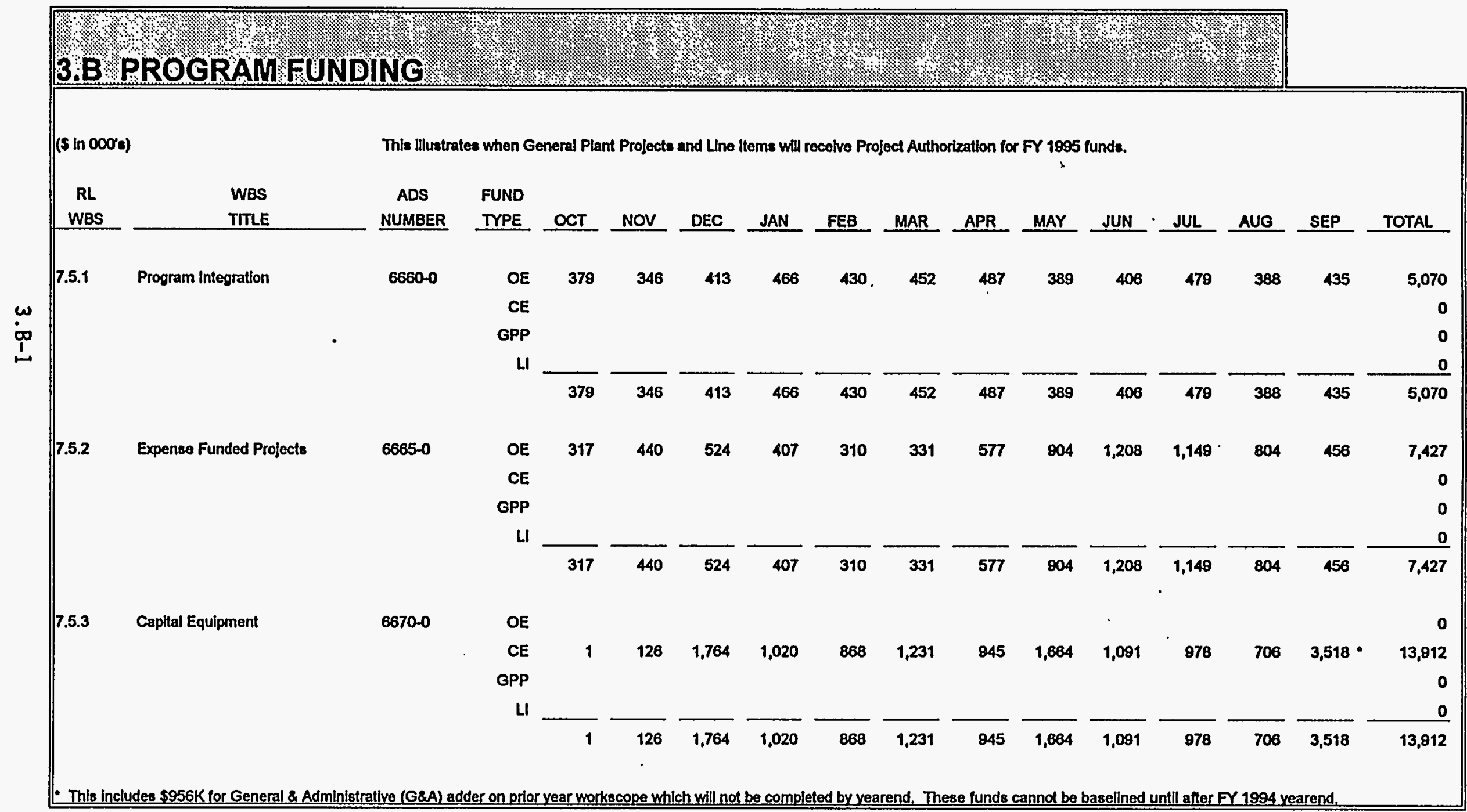




\section{LANDLORD PROGRAM}

\section{5}

\section{FY 1995 MYPP}

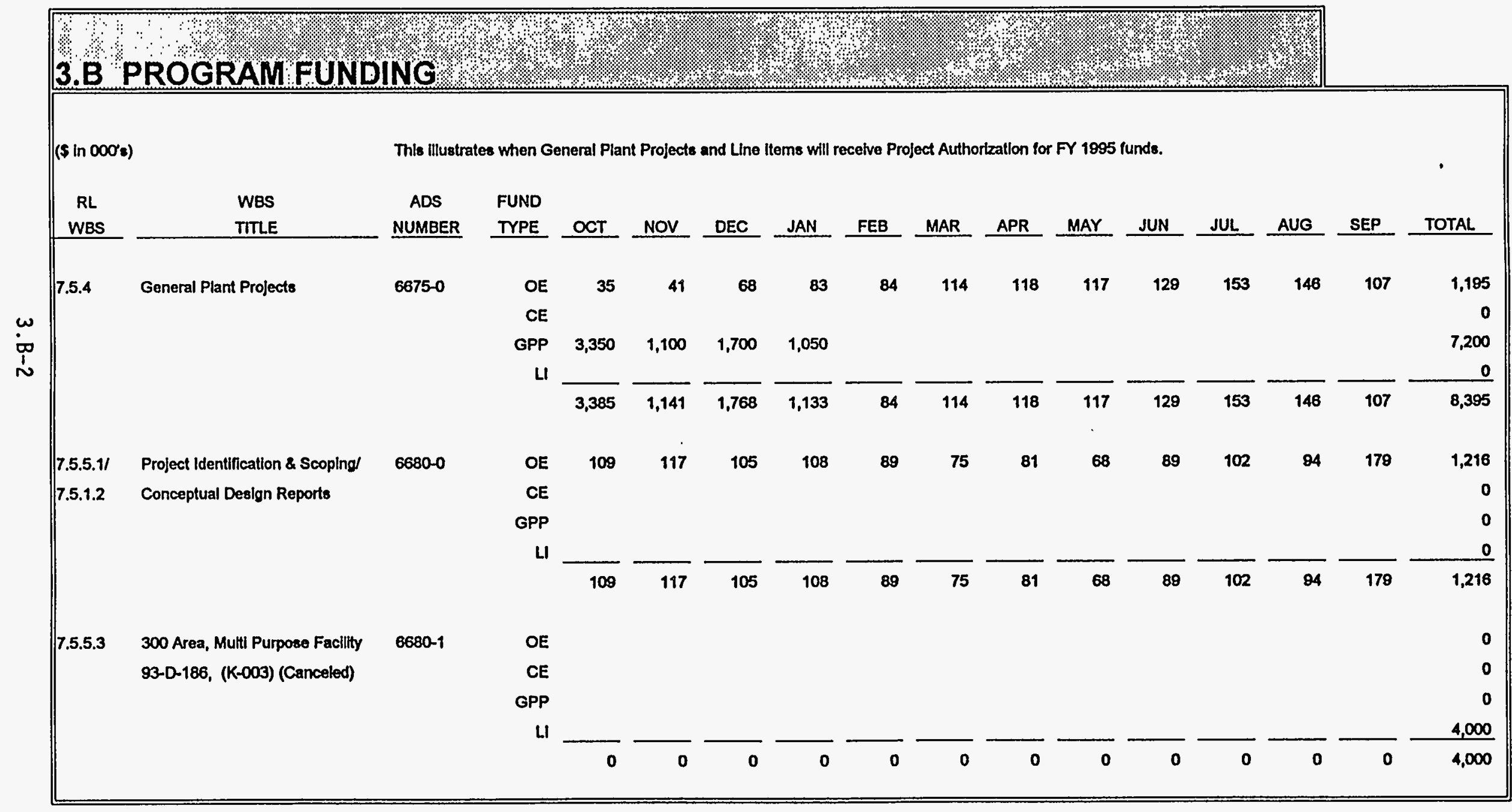




\section{LANDLORD PROGRAM}

\section{5}

\section{FY 1995 MYPP}

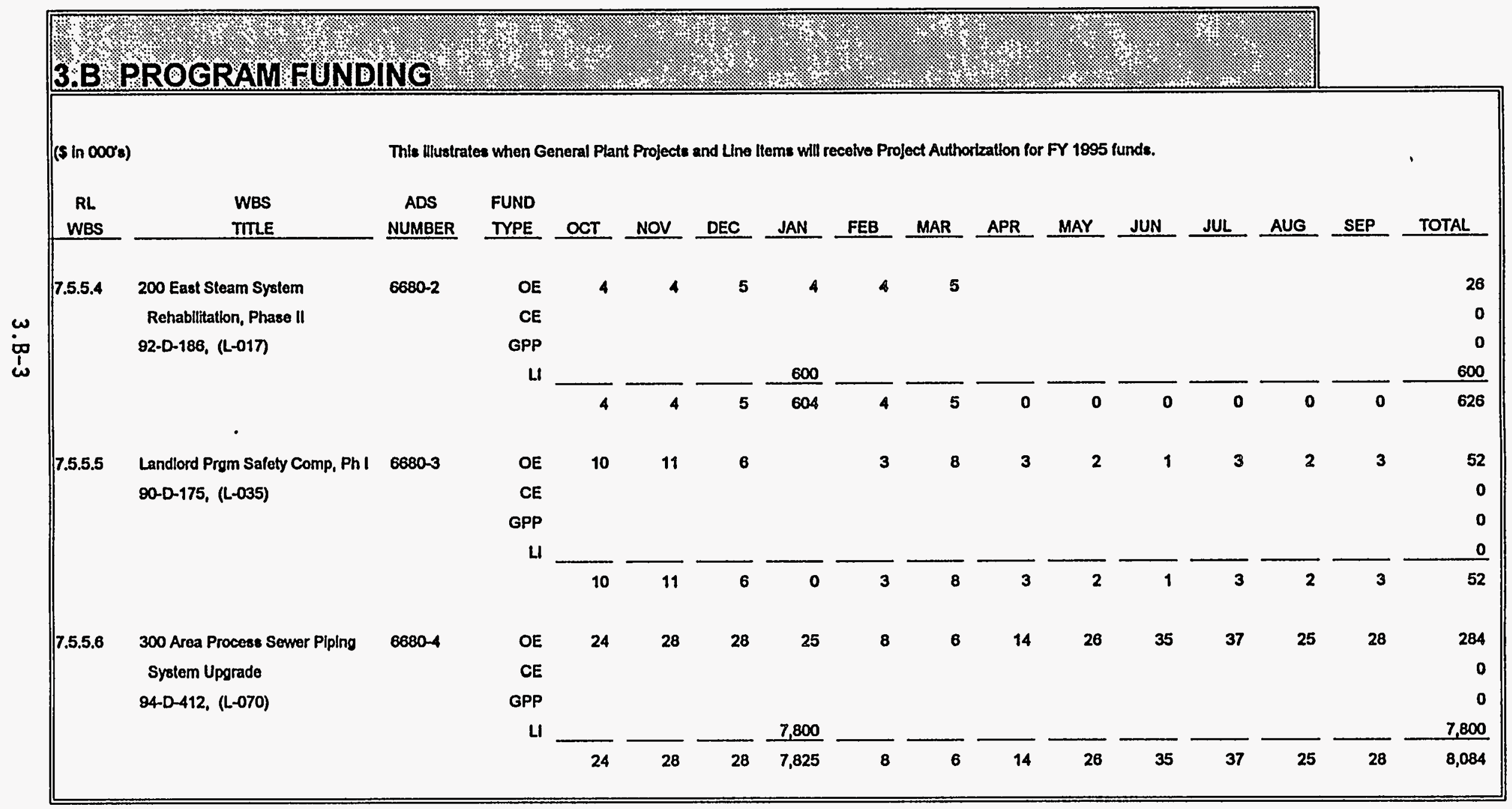




\section{LANDLORD PROGRAM}

\section{5}

\section{FY 1995 MYPP}

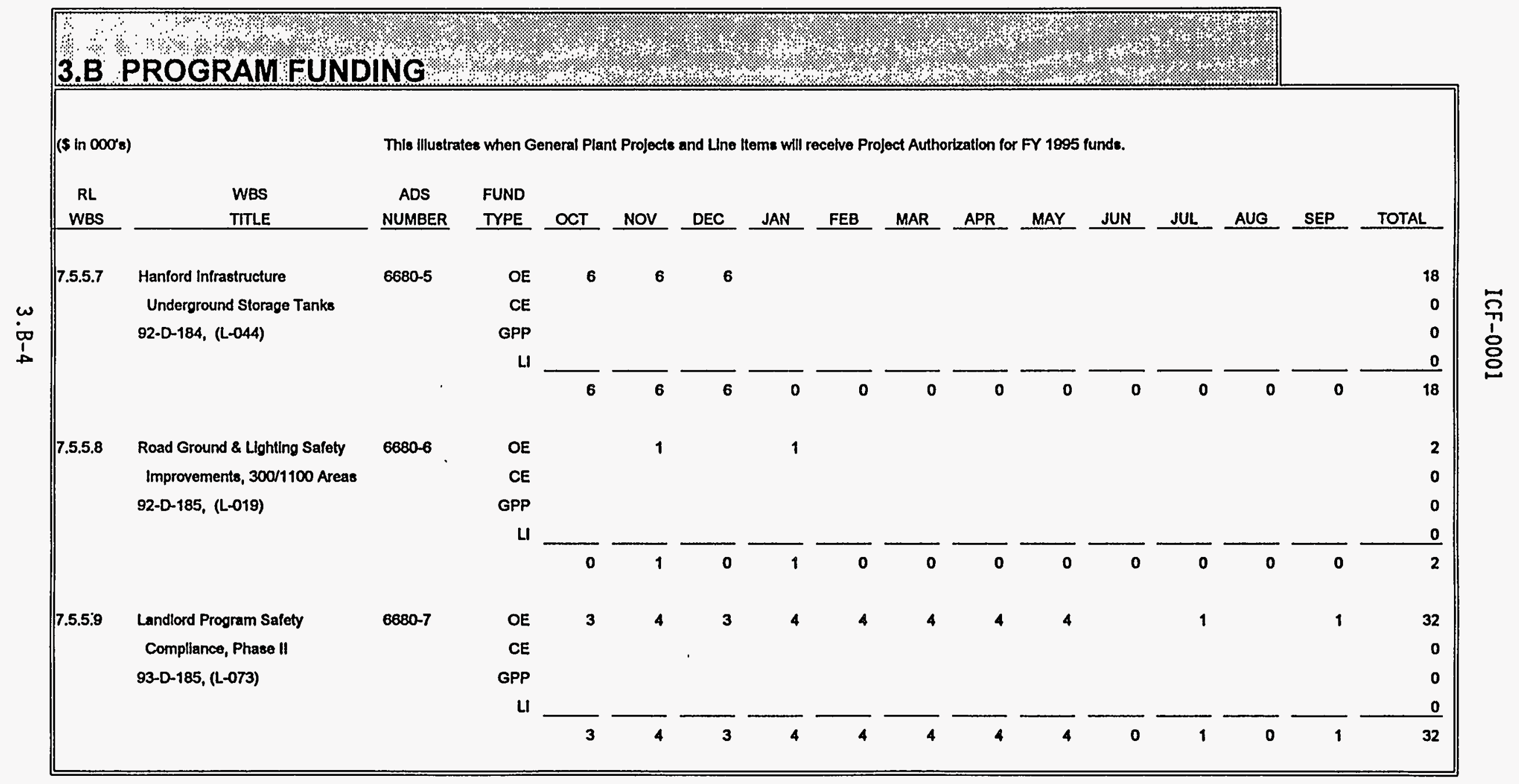




\section{LANDLORD PROGRAM}

\section{5}

\section{FY 1995 MYPP}

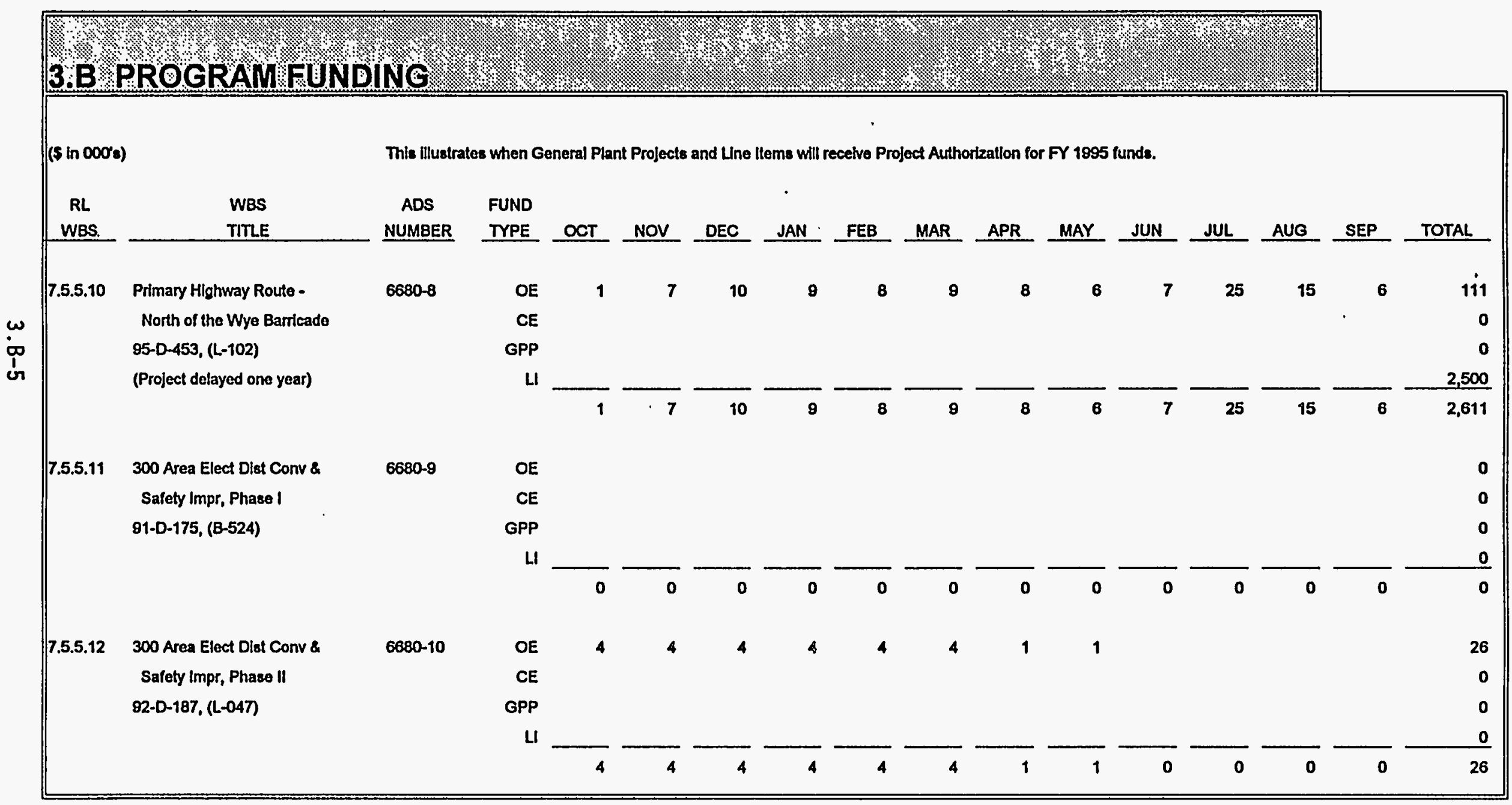




\section{LANDLORD PROGRAM}

\section{5}

\section{FY 1995 MYPP}

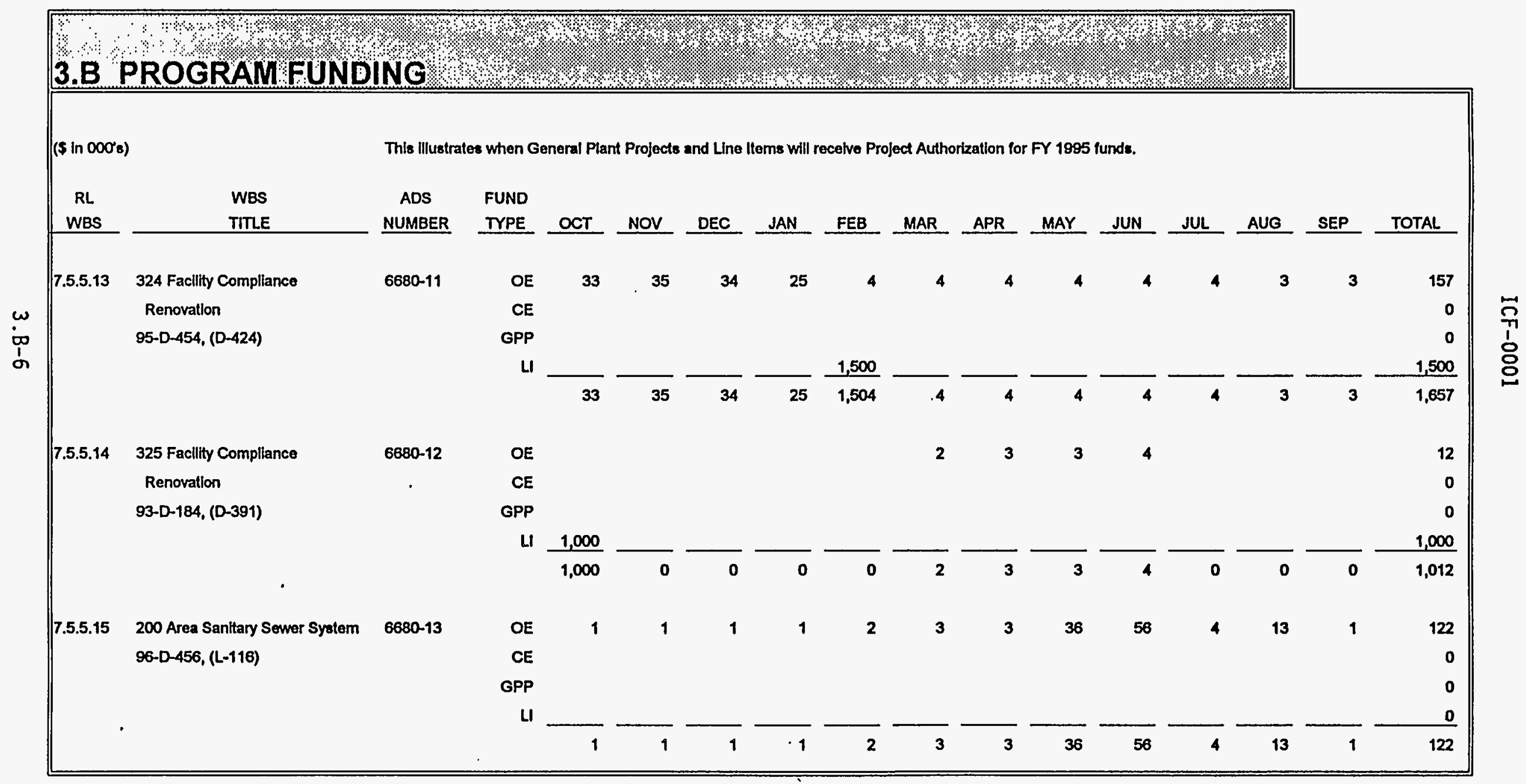




\section{LANDLORD PROGRAM}

\section{5}

\section{FY 1995 MYPP}

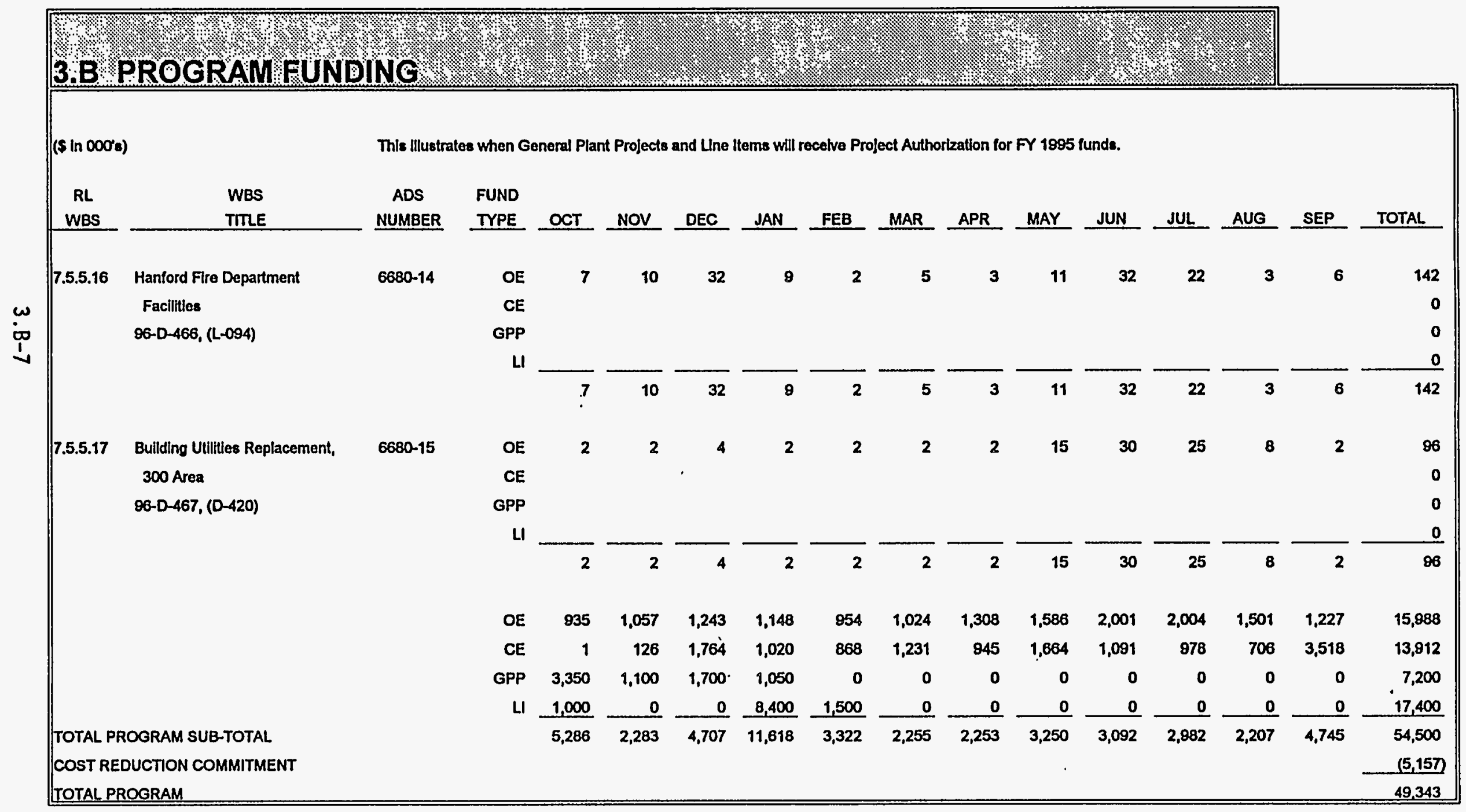




\section{LANDLORD PROGRAM}

\section{5}

\section{FY 1995 MYPP}

\section{C PROGRAMIFUNDING ADSIFUND TYPE}

\begin{tabular}{|c|c|c|c|c|c|c|c|c|c|c|c|}
\hline \multirow[b]{2}{*}{$\mathbf{R L}$} & \multirow[b]{2}{*}{$\begin{array}{l}\text { WBS } \\
\text { TITLE }\end{array}$} & \multirow[b]{2}{*}{ ADS } & \multicolumn{2}{|c|}{$\begin{array}{l}\text { OPERATING } \\
\text { EXPENSE } \\
\end{array}$} & \multicolumn{2}{|c|}{ CAPITAL EQUIPMENT } & \multicolumn{2}{|c|}{ GPP } & \multicolumn{2}{|c|}{$\begin{array}{l}\text { LINE } \\
\text { ITEM } \\
\end{array}$} & \multirow[b]{2}{*}{ TOTAL } \\
\hline & & & $\begin{array}{l}\text { FY95 } \\
\text { BA }\end{array}$ & $\begin{array}{l}\text { FY94 } \\
\text { Projected } \\
\text { Carryover }\end{array}$ & $\begin{array}{c}\text { FY95 } \\
\text { BA } \\
\end{array}$ & $\begin{array}{c}\text { FY94 } \\
\text { Projected } \\
\text { Carryover } \\
\end{array}$ & $\begin{array}{l}\text { FY95 } \\
\text { BA }\end{array}$ & $\begin{array}{l}\text { FY94 } \\
\text { Projected } \\
\text { Carryover }\end{array}$ & $\begin{array}{c}\text { FY95 } \\
\text { BA } \\
\end{array}$ & $\begin{array}{c}\text { FY94 } \\
\text { Projected } \\
\text { Carryover }\end{array}$ & \\
\hline 7.5 .1 & Program Integration & $6660-0$ & 5,070 & 916 & & & & & & 1,290 & 7,276 \\
\hline 7.5 .2 & Expense Funded Projects & $6665-0$ & 7,427 & 1,546 & & & & & & & 8,973 \\
\hline 7.5 .3 & Capital Equipment & $6670-0$ & & & 13,912 & 5,447 & & & & & 19,359 \\
\hline 7.5 .4 & General Plant Projects & $6675-0$ & 1,195 & & & & 7,200 & 13,094 & & & 21,489 \\
\hline $\begin{array}{l}7.5 .5 .1 / \\
7.5 .5 .2\end{array}$ & $\begin{array}{l}\text { Project Identification \& Scoping/ } \\
\text { Conceptual Design Reports }\end{array}$ & $6680-0$ & 1,216 & 54 & & & & & & & 1,270 \\
\hline 7.5.5.3 & $\begin{array}{l}300 \text { Area, Mutt Purpose Facility } \\
\text { 93-D-186, (K-003) (Canceled) }\end{array}$ & $6680-1$ & & & & & & & 4,000 & & 4,000 \\
\hline 7.5.5.4 & $\begin{array}{l}200 \text { East Steam System Rehab., } \\
\text { Phase II 92-D-186, (L-017) }\end{array}$ & $6680-2$ & 26 & & & & & & 600 & 917 & 1,543 \\
\hline 7.5.5.5 & $\begin{array}{l}\text { Landlord Prgm Safety Comp, Ph I } \\
90-D-175,(L-035)\end{array}$ & $6680-3$ & 52 & & & & & & & 14,876 & 14,928 \\
\hline 7.5.5.6 & $\begin{array}{l}300 \text { Area Process Sewer Piping } \\
\text { System 94-D-412, (L-070) }\end{array}$ & $6680-4$ & 284 & & & & & & 7,800 & 761 & 8,845 \\
\hline 7.5.5.7 & $\begin{array}{l}\text { Hanford Infrast. Underground } \\
\text { Storage Tanks, 92-D-184. (L-044) }\end{array}$ & $6680-5$ & 18 & & & & & & & 1,752 & 1,770 \\
\hline
\end{tabular}




\section{LANDLORD PROGRAM}

\section{5}

\section{FY 1995 MYPP}

\begin{tabular}{|c|c|c|c|c|c|c|c|c|c|c|c|}
\hline$\left(s \ln 000^{\circ}\right.$ & & , & \multicolumn{2}{|c|}{$\begin{array}{l}\text { OPERATING } \\
\text { EXPENSE } \\
\end{array}$} & \multicolumn{2}{|c|}{ CAPITAL EQUIPMENT } & \multicolumn{2}{|c|}{ GPP } & \multicolumn{2}{|c|}{$\begin{array}{l}\text { LINE } \\
\text { ITEM }\end{array}$} & \\
\hline $\begin{array}{c}\text { RL } \\
\text { WBS }\end{array}$ & $\begin{array}{l}\text { WBS } \\
\text { TITLE } \\
\end{array}$ & $\begin{array}{c}\text { ADS } \\
\text { NUMBER }\end{array}$ & $\begin{array}{l}\text { FY95 } \\
\text { BA }\end{array}$ & $\begin{array}{c}\text { FY94 } \\
\text { Projected } \\
\text { Carryover }\end{array}$ & $\begin{array}{c}\text { FY95 } \\
\text { 8A } \\
\end{array}$ & $\begin{array}{l}\text { FY94 } \\
\text { Projected } \\
\text { Carryover } \\
\end{array}$ & $\begin{array}{l}\text { FY95 } \\
\text { BA }\end{array}$ & $\begin{array}{l}\text { Fr94 } \\
\text { Projected } \\
\text { Carrover }\end{array}$ & $\begin{array}{c}\text { FY95 } \\
\text { BA }\end{array}$ & $\begin{array}{l}\text { FY94 } \\
\text { Projected } \\
\text { Camyover } \\
\end{array}$ & TOTAL \\
\hline 7.5 .5 .8 & $\begin{array}{l}\text { Road, Gmd \& Lighting Safety } \\
\text { Improvements, 300/1100 Areas } \\
\text { 92-D-185, (L-019) }\end{array}$ & $6680-6$ & 2 & & & & & & & 1,630 & 1,632 \\
\hline 7.5.5.8 & $\begin{array}{l}\text { Landlord Prgm Safety Comp, Ph II } \\
\text { 93-D-185, (L-073) }\end{array}$ & $6680-7$ & 3: & & & & & & & 1,372 & 1,404 \\
\hline 7.5.5.10 & $\begin{array}{l}\text { Primary Highway Rte - North of } \\
\text { Wye Barricade 95-D-453, (L-102) } \\
\text { (Project Delayed one Year) }\end{array}$ & $6680-8$ & 11 & & & & & & 2,500 & & 2,611 \\
\hline 7.5.5.11 & $\begin{array}{l}300 \mathrm{~A} \text { Elec Dist Conv \& Safety } \\
\text { Improve., Ph I 91-D-175, (B-524) }\end{array}$ & $6680-9$ & & & & & & & & 2,030 & 2,030 \\
\hline 7.5.5.12 & $\begin{array}{l}\text { 300A Elec Dist Conv \& Safety } \\
\text { Improve., Ph II 92-D-187, (L-047) }\end{array}$ & $6680-10$ & 2 & & & & & & & 8,378 & 8.404 \\
\hline 7.5.5.13 & $\begin{array}{l}324 \text { Facillty Compllancel } \\
\text { Renovation } 95-D-454,(D-424)\end{array}$ & $6680-11$ & 15 & & & & & & 1,500 & & 1,657 \\
\hline 7.5.5.14 & $\begin{array}{l}325 \text { Facility Compllance/ } \\
\text { Renovation } 83-D-184, \text { (D-391) }\end{array}$ & $6680-12$ & 12 & & & & & & 1,000 & 3,060 & 4,072 \\
\hline 7.5.5.15 & $\begin{array}{l}200 \text { Area Sanitary Sewer System } \\
\text { Renovation } 96-D-456,(L-116)\end{array}$ & $6680-13$ & 12 & & & & & & & & 122 \\
\hline
\end{tabular}




\section{LANOLORD PROGRAM}

\section{5}

\section{FY 1995 MYPP}

\section{C PROGRAM FUNDING - ADS/FUND TYPE}

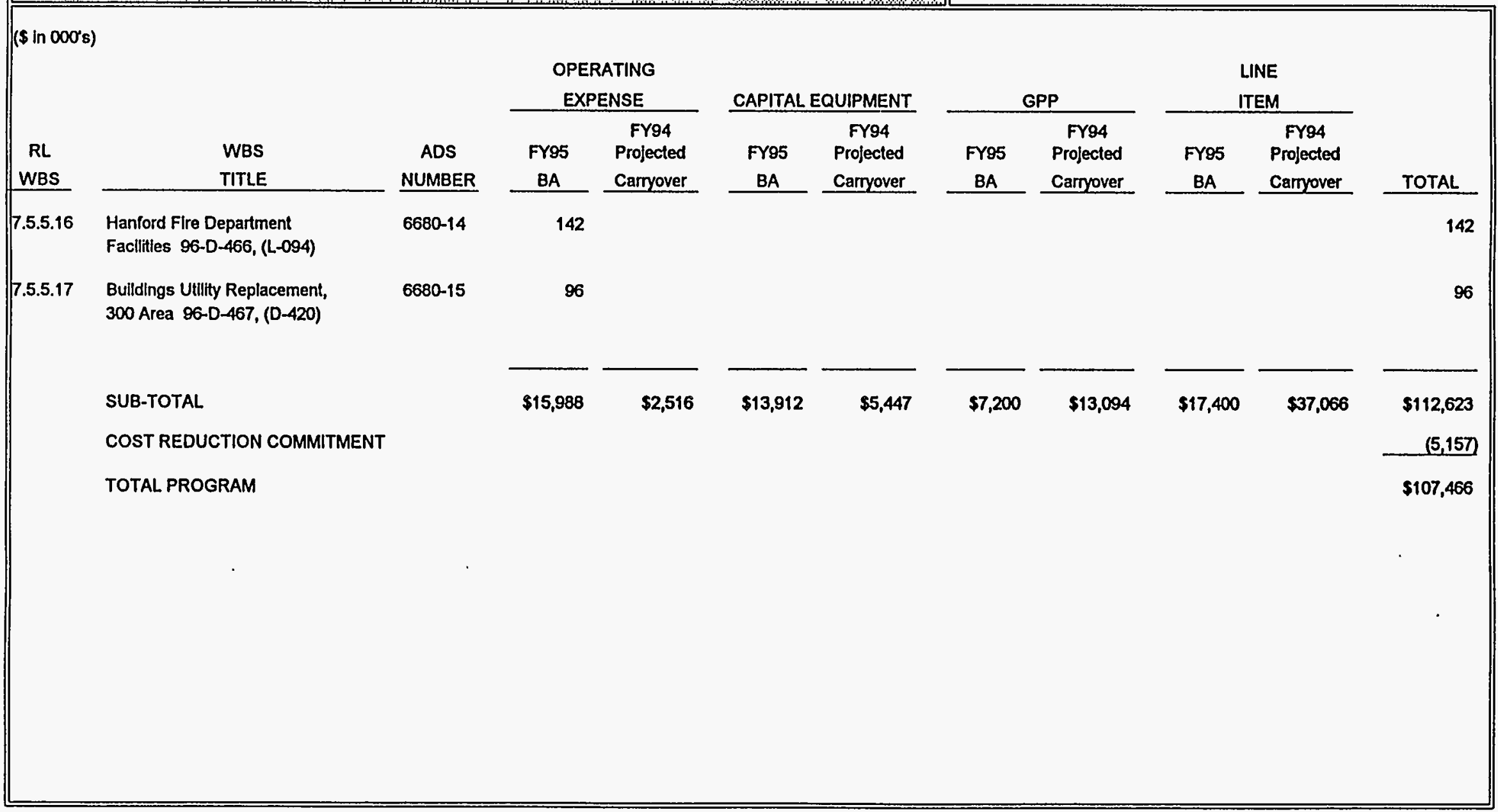

